\title{
IntechOpen
}

\section{Some Advanced Functionalities of Optical Amplifiers}

Edited by Sisir Kumar Garai

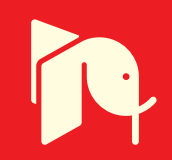





\section{SOME ADVANCED \\ FUNCTIONALITIES OF OPTICAL AMPLIFIERS}

Edited by Sisir Kumar Garai 


\section{Some Advanced Functionalities of Optical Amplifiers}

http://dx.doi.org/10.5772/60493

Edited by Sisir Kumar Garai

\section{Contributors}

George T. Kanellos, Theoni Alexoudi, Stefanos Dris, Dimitrios Kalavrouziotis, Paraskevas Bakopoulos, Amalia Miliou, Nikos Pleros, Chien-Hung Yeh, Ali Rostami, Ghasem Rostami, Mahboubeh Dolatyari, Khalil Safari, Rintaro Koda, Hideki Watanabe, Shunsuke Kono, Christos Vagionas, Jan Bos, Juan A. Vallés, Ramona Gălătuş, Guilhem De Valicourt, Miquel Angel Mestre Adrover, Nikolay Moroz, Yvan Pointurier

\section{(c) The Editor(s) and the Author(s) 2015}

The moral rights of the and the author(s) have been asserted.

All rights to the book as a whole are reserved by INTECH. The book as a whole (compilation) cannot be reproduced, distributed or used for commercial or non-commercial purposes without INTECH's written permission.

Enquiries concerning the use of the book should be directed to INTECH rights and permissions department (permissions@intechopen.com).

Violations are liable to prosecution under the governing Copyright Law.

\section{(cc) BY}

Individual chapters of this publication are distributed under the terms of the Creative Commons Attribution 3.0 Unported License which permits commercial use, distribution and reproduction of the individual chapters, provided the original author(s) and source publication are appropriately acknowledged. If so indicated, certain images may not be included under the Creative Commons license. In such cases users will need to obtain permission from the license holder to reproduce the material. More details and guidelines concerning content reuse and adaptation can be foundat http://www.intechopen.com/copyright-policy.html.

\section{Notice}

Statements and opinions expressed in the chapters are these of the individual contributors and not necessarily those of the editors or publisher. No responsibility is accepted for the accuracy of information contained in the published chapters. The publisher assumes no responsibility for any damage or injury to persons or property arising out of the use of any materials, instructions, methods or ideas contained in the book.

First published in Croatia, 2015 by INTECH d.o.o.

eBook (PDF) Published by IN TECH d.o.o.

Place and year of publication of eBook (PDF): Rijeka, 2019.

IntechOpen is the global imprint of IN TECH d.o.o.

Printed in Croatia

Legal deposit, Croatia: National and University Library in Zagreb

Additional hard and PDF copies can be obtained from orders@intechopen.com

Some Advanced Functionalities of Optical Amplifiers

Edited by Sisir Kumar Garai

p. cm.

ISBN 978-953-51-2237-1

eBook (PDF) ISBN 978-953-51-6649-8 


\section{We are IntechOpen, \\ the world's leading publisher of Open Access books}

Built by scientists, for scientists

\section{$3,800+$}

Open access books available

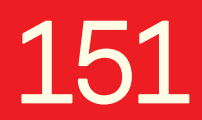

Countries delivered to

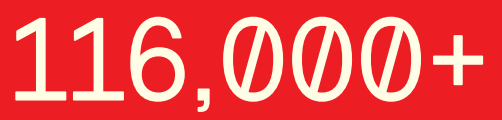

International authors and editors
$120 \mathrm{M}+$

Downloads

Our authors are among the

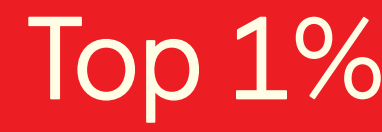

most cited scientists

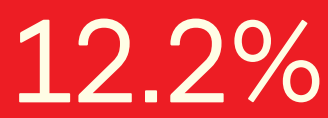

Contributors from top 500 universities

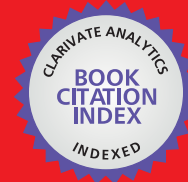

WEB OF SCIENCE ${ }^{\mathrm{TM}}$

Selection of our books indexed in the Book Citation Index in Web of Science ${ }^{\mathrm{TM}}$ Core Collection (BKCI)

Interested in publishing with us?

Contact book.department@intechopen.com

Numbers displayed above are based on latest data collected.

For more information visit www.intechopen.com

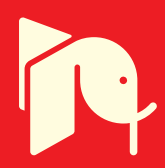





\section{Meet the editor}

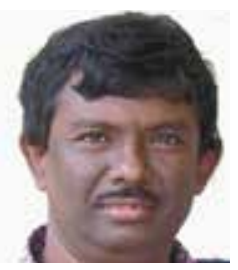

Sisir Kumar Garai was born in 1971 in West Bengal, India. He received his M.Sc. degree in Physics in 1994 and M.Tech. degree in Microwaves in 1998 and was awarded his Ph.D. degree in Science (Physics) in 2012 from The University of Burdwan, West Bengal, India. His fields of research interest include optoelectronics, optical computation and communication, and he has contributed more than forty papers in various national and international journals and edited a book entitled Selected Topics on Optical Amplifiers in Present Scenario (ISBN 978-953-51-0391-2; InTech; March 3, 2012). He received the best paper presentation award in the 16th West Bengal State Science and Technology Congress (2009), and his name is also included in Marquis Who's Who (2010 and 2011) and by the International Biographical Centre (IBC), Cambridge, 2011. Dr. Garai is the author of the book Fundamentals of Frequency Encoded Optical Logic Processors (LAP Lambert Academic Publishing, Germany, 2015). He has been an Assistant Professor in the Department of Physics at the M.U.C. Women's College, Burdwan, West Bengal, India, since 2002. 



\section{Contents}

Preface XI

Chapter 1 Efficient and Validated Time Domain Numerical Modeling of Semiconductor Optical Amplifiers (SOAs) and SOA-based Circuits 1

Christos Vagionas, Jan Bos, George T. Kanellos, Nikos Pleros and Amalia Miliou

Chapter 2 Gallium Nitride-based Semiconductor Optical Amplifiers 27 Rintaro Koda, Hideki Watanabe and Shunsuke Kono

Chapter 3 Semiconductor Optical Amplifier for Next Generation of High Data Rate Optical Packet-Switched Networks 47

Guilhem de Valicourt, Miquel Angel Mestre Adrover, Nikolay D. Moroz and Yvan Pointurier

Chapter 4 All-optical Semiconductor Optical Amplifiers Using Quantum Dots (Optical Pumping) 85

Khalil Safari, Ali Rostami, Ghasem Rostami and Mahboubed Dolatyari

Chapter 5 Semiconductor Optical Amplifier (SOA)-Based Amplification of Intensity-Modulated Optical Pulses - Deterministic Timing Jitter and Pulse Peak Power Equalization Analysis 109 T. Alexoudi, G.T. Kanellos, S. Dris, D. Kalavrouziotis, P. Bakopoulos, A. Miliou and N. Pleros

Chapter 6 Investigation of Broadband S-Band to L-Band Erbium-Doped Fiber Amplifier (EDFA) Module 129

Chien-Hung Yeh 
Chapter 7 Optimized Design of Yb3+/Er3+-Codoped Phosphate Microring Resonator Amplifiers 149

Juan A. Vallés and R. Gălătuş 


\section{Preface}

To provide Internet-based communication network, the speed of computing as well as of data processing should be high enough with a transmission medium of enormous potential bandwidth having the capability to handle vast amount of information. On the other hand, conventional electronic technology has already reached its ultimate speed limit $(40 \mathrm{~Gb} / \mathrm{s})$ through the limitation of miniaturization of chips and bandwidth limitation, and obviously it creates data traffic jam in Internet-based networking services. Therefore, a drastic solution to this acute problem is needed, and scientists and technologists promote their thinking to a totally different track from the conventional electronic system so that we can further improve on the computer performance and data signal processing with a potential communicating medium to such an extent that we will be well prepared to accept the present and future challenges of data traffic.

Optical computing and optical signal processing are strongly believed to be the most feasible technology that can provide the way out of the extreme limitations imposed on the speed and the complexity of present-day computation and communication by conventional electronics. Optics provides higher bandwidth than electronics, which enables more information to be carried simultaneously and data to be processed in parallel with impressive increase in speed by several orders of magnitude over that of the electronic signals. If the parallelism of optics were associated with fast switching speed of optical devices, it would result in surprising computational speed and processing of data.

Considering the present scenario of speed and bandwidth limitations of electronic computing, signal processing and the future problem of data traffic, scientists, technologists and researchers are working in the field of all-optical data and signal processing. The role of the optical amplifier is indispensable in such optical communication network. Over the past two decades, optical amplifiers such as erbium-doped fibre amplifier (EDFA), Raman amplifier and semiconductor optical amplifier (SOA) are the prime optical network functional components and have many functional applications, such as wavelength conversion, regeneration, wavelength selection, booster, in-line amplification, in-node optical preamplification and mid-span spectral inversion.

The topics selected in this book cover some advanced functionalities of SOAs in the field of optical communication network. This book also includes the role of broadband S-band to Lband EDFA in WDM communication network, and it also covers the optimized design technique of $\mathrm{Yb}^{3+} / \mathrm{Er}^{3+}$-codoped phosphate microring resonator amplifiers.

The book contains seven chapters. The first chapter deals with an efficient and validated time-domain numerical modelling of SOAs and SOA-based circuits; the second chapter is based on the working of gallium nitride-based SOAs. The role of SOAs for the next genera- 
tion of high-data-rate optical packet-switched network is presented in Chapter 3. Chapter 4 deals with the all-optical semiconductor optical amplifier based on quantum dots (QD-SOA) and its function as an arithmetic processor. In Chapter 5, the authors have presented the role of SOAs in intensity modulation of the optical pulses and their use in deterministic timing jitter and peak pulse power equalization analysis. In Chapter 6, the investigation of broadband S-band to L-band EDFA module is presented, and Chapter 7 includes the optimized design technique of $\mathrm{Yb}^{3+} / \mathrm{Er}^{3+}$-codoped phosphate microring resonator amplifiers.

All the topics selected for this book are very interesting and well organized, and the presentation is also very lucid. This book covers the emerging applications of optical amplifiers in the present scenario, and I believe that the book will be of great value to the researchers in the field of optical communication, component suppliers, postgraduate students, academics and anyone seeking to understand the trends of optical amplifiers in the present scenario and the consequent changes in optical amplifier design and technology.

Without the unstinting support from so many persons, it would not have been possible for me to edit this book. Therefore, I feel compelled to express my gratitude to all of them. First of all, I would like to express my indebtedness to all the writers of this book for contributing their valuable research works. Their patience and insights from which I have learnt will surely benefit me for the rest of my life. I am very grateful to Ms. Iva Simcic, Publishing Process Manager, for her incessant support in numerous ways to enable me to do the editorial work. I wish to convey my thanks to the technical editorial staff and all other staff members of the publisher. I am grateful to my family as well for their constant encouragement and support. Finally, I would like to extend my sincere thanks to all my colleagues at M.U.C. Women's College, Burdwan, for their incessant encouragement.

We shall deem our effort amply rewarded if the book wins the appreciation of the users.

Dr. Sisir Kumar Garai

Assistant Professor of Physics

M.U.C. Women's College, Burdwan

West Bengal, India 


\title{
Chapter 1
}

\section{Efficient and Validated Time Domain Numerical Modeling of Semiconductor Optical Amplifiers (SOAs) and SOA-based Circuits}

\author{
Christos Vagionas, Jan Bos, George T. Kanellos, Nikos Pleros and \\ Amalia Miliou
}

Additional information is available at the end of the chapter

http://dx.doi.org/10.5772/61801

\begin{abstract}
Semiconductor optical amplifiers (SOAs) have been extensively used in a wealth of telecom and datacom applications as a powerful building block that features large optical gain, all-optical gating function, fast response, and ease of integration with other functional semiconductor devices. As fabrication technologies are steadily maturing toward enhanced yield, SOAs are foreseen to play a pivotal role in complex photonics integrated circuits (PICs) of the near future. From a design standpoint, accurate numerical modeling of SOA devices is required toward optimizing PICs response from a system perspective, while enhanced circuit complexity calls for efficient solvers. In this book chapter, we present established experimentally validated SOA numerical modeling techniques and a gain parameterization procedure applicable to a wide range of SOA devices. Moreover, we describe multigrid concepts and implicit schemes that have been only recently presented to SOA modeling, enabling adaptive time stepping at the SOA output, with dense sampling at transient phenomena during the gain recovery and scarce sampling during the steady-state response. Overall, a holistic simulation methodology approach along with recent research trends are described, aiming to form the basis of further developments in SOA modeling.
\end{abstract}

Keywords: Semiconductor optical amplifier, numerical modeling, transfer matrix method, multigrid techniques

\section{Introduction}

The explosive growth of information traffic and the concomitant increase of bandwidth hungry applications have contributed to a growing demand for communications networks offering 
greater bandwidth and flexibility at lower cost [1-3]. This has led to a series of technological advances in high-speed backbone networks and telecommunications, where integrated optical systems delivering low-cost, low-power, high-bandwidth transmission, and high-speed switching elements are increasingly required [4]. Toward meaningful and functional subsystems, high-speed all-optical elements such as all-optical signal wavelength conversion [5], on/ off keying modulation [6,7], header recognition [8], optical buffering [9], and signal regeneration [10] have been developed, targeting to perform basic functionalities in the optical domain. Meanwhile, photonic integration technologies have steadily matured over the last decade, achieving improved yield fabrication [11,12], allowing the fabrication of complex single-chip photonic modules with advanced functionality. This has resulted in impressive demonstrations of functional complex circuits and switching architectures [12-17], making firm steps toward medium-scale (MS) photonic integrated chips (PICs).

On the way to develop complex devices with enhanced processing capabilities, semiconductor optical amplifiers (SOAs) have been a powerful building block that provides high-speed alloptical switching operations [18] by featuring large optical gain, optical gating function, controllable performances by injection current, compactness, fast response, and ease of integration with other functional semiconductor devices [18]. SOAs are utilized in telecom $[19,20]$ and datacom $[21,22]$ PICs either as single SOA travelling waveguides, supporting crossgain modulation (XGM) phenomena, or arranged in SOA-Mach-Zehnder interferometer (SOA-MZI) configurations, supporting cross-phase modulation (XPM) phenomena. The reason lies in the maturity of the SOA technology to a point where commercial devices are readily available either as bulk single chip elements [23-25], in arrays of certain pitch [26,27], or packaged in discrete fiber pigtailed components [28] for use in optical communication systems. Moreover, with recent research achievements on mid-board flip-chip bonding of an SOA array to a silicon-on-insulator (SOI) platform [27] or the development of temperature stable SOA [25], SOAs are expected to play a pivotal role in future PICs.

In this regime, SOA numerical modeling has attracted a lot of research attention during the last decades, as mathematical models are required to aid in the design of SOAs and to predict their operational characteristics [29-36]. During the development process and prior to fabrication, SOA models that take into account the semiconductor properties of the active material and cross-sectional dimensions are employed when optimizing the amplification gain and output power over a wideband steady state and under various external current injections. In addition, during the system-level performance study of SOA-based circuits, time domain simulations are required for an accurate evaluation of the circuit response with multiple signals propagating bidirectionally along the waveguide. With growing complexity of circuits, system level designs call for experimentally validated yet efficient solvers that support multiple signals at different wavelengths propagating in multiple directions.

This book chapter aims to provide a holistic simulation methodology approach for efficient and validated numerical modeling of SOAs and SOA-based circuits in the time, which may form the basis of further developments in SOA modeling. This chapter is organized as follows: a short review of established SOA modeling approaches in the literature will be presented in Section 2, along with an overview of some recent research efforts. In Section 3, a description 
of an experimentally validated time domain SOA model relying on the TMM will be presented [37-41], relying on explicit modeling techniques, followed by a gain parameterization procedure for tailoring the TMM model to characterization measurements of other SOA devices in Section 4. Having developed an experimentally validated SOA model, we numerically investigate the impact of the external current injection, the SOA length, and the light propagation direction in the gain recovery using pump-probe techniques to qualitatively study the shortening of the gain recovery time toward achieving high bandwidth switching operations. Section 5 presents a newly introduced efficient solver in the time domain, relying on implicit schemes and multigrid concepts. Implicit schemes allow developing an adaptive step sizecontrolled solver for the WDM SOA response [42-44], which extends the validated TMM model by lifting the limitations of spatiotemporal grids and releasing adaptive sampling at the SOA output. Toward application in realistic system-level scenarios, the last section presents experimentally validated numerical results for two predominant SOA-based all-optical signal processing circuits, such as a coupled SOA XGM flip-flop arrangement and an XOR gate based on SOA-MZI XPM configuration. The former acts as memory for sequential logic processing circuits, while the latter forms a basic building block of combinational logic signal processing circuits. Conclusions are addressed in the final section.

\section{Established SOA numerical models}

SOA numerical modeling has progressed on multiple fonts during the last decades, and various simulations approaches have been developed [29-36]. SOA models can be roughly divided in two categories: (i) the material models that target optimization of the emission properties, before and during the development-fabrication process of an SOA device and (ii) the circuit models, which account for the carrier density, the interband, and the intraband phenomena for simulations of light-matter interaction in realistic system-level application scenarios.

The material models have long studied the gain coefficients, emission characteristics, and spectral properties of the active material. Optimization parameters include the peak gain; the molecular fractions of the materials in the semiconductor compounds; the cross-sectional dimensions, e.g., ridge width, core height, etc.; and the structural properties, e.g., number of quantum wells (QW), quantum dots (QD), and so on. Gillner et al. [45] investigated longwavelength semiconductor laser amplifiers by means of an experimentally validated SOA model and considering different structural parameters such as thickness of the active layer and amplifier length. The model that takes into account Auger recombination, thermal effects, and spontaneous emission was developed in order to optimize the spectral gain properties of SOA lasers, such as peak gain wavelength shift and width of gain curve. Interestingly, it was shown that there exists an optimum active layer thickness with respect to current density for a certain gain, while increased SOA length allows higher gains with reduced wavelength variation of the peak gain. Similarly, a comprehensive model was presented by Minch et al. [46] for the calculation of the band edge profile of both the $\operatorname{In}_{1-x} \mathrm{Ga}_{x} \mathrm{As}_{y} \mathrm{P}_{1-y}$ and $\operatorname{In}_{1-x-y} \mathrm{Ga}_{x} \mathrm{Al}_{y} \mathrm{As}$ quantum-well systems with an arbitrary composition, as typical semiconductor compounds 
found in SOAs. This model provided in-depth knowledge with mathematical curve fits between the measured net modal gains for both material systems and calculations from the realistic band structure, including valence band mixing effects, allowing to extract the relationship between total current density and carrier density. More recently, research has focused on studying the properties of newly introduced active compound materials, such as the GaInNAs. The analysis of the broadband gain of a GaInNAs single quantum-well (QW) SOA that takes into account the tunability of the gain and incorporates quantum dot (QD) fluctuations due to compositional fluctuations of $\mathrm{N}$ within the QW is presented by Xiao et al. [47]. Finally, III/V-on-SOI bonding processes have also been into design consideration recently, with a comprehensive model presented by Cheung et al. [48] providing valuable insight to the design requirements of the optimization process of the structural and material parameters, e.g., the width, composition, and number of quantum wells of a compressively strained $\operatorname{In}(1-$ $x-y) \mathrm{Ga}(x) \mathrm{Al}(y)$ As quantum-well active region for emission at approximately $1550 \mathrm{~nm}$.

This chapter focuses more on the 2nd category, toward presenting a holistic simulation approach of time domain numerical modeling of circuit-level simulations. An advanced time domain dynamic numeric model that accounts for the ultrafast gain dynamics, including the intraband phenomenon, the gain saturation, and the gain spectral profile of an SOA, has been presented by Toptchiyski et al. [49], where the model is employed in a system-level simulation to investigate the gain dynamics of the light-propagation direction during pump probe measurement and application in an Sagnac interferometer switch semiconductor laser amplifier in a loop mirror (SLALOM). By exploiting the carrier density fluctuation due to interband phenomena within the SOA, many applications have been demonstrated already. Accurate designs and numerical analysis of various SOA-based devices have resulted in successful demonstrations of optical flip-flops and random access memory (RAM) cells, or SOA-Mach-Zehnder interferometers (MZIs) and cutting edge optical routing devices, profiting from cross-gain modulation (XGM) or cross-phase modulation (XPM) phenomena [38]. Meanwhile, circuit-level modeling approaches have been developed for experimental verification and accuracy.

Traditional time domain SOA models in principle treat the gain dynamics based on the longitudinal carrier distribution along the device, relying on the free carrier rate equation for the electron-photon interaction. To achieve this, a fine longitudinal SOA discretization is usually adopted. Dividing the SOA into cascaded elementary sectors of equally small propagation length and time stepping allows applying wideband steady-state material gain coefficients [31] across the emission spectrum, using a one-by-one space and time representation of the carrier densities and photon fluxes. This discretization derives from an explicit scheme for solving the associated differential equations, resulting in an equidistant grid with constant time stepping. This technique is the standard approach used in circuit-level designs and has proven capable of delivering quantitative matching with experimental measurements [30-35]. Following this concept, an experimentally validated time domain SOA model relying on the TMM analysis technique was developed by Vagionas et al. [37] with the simulation results coming in close agreement with the characterization measurements on a commercial SOA device in terms of the emission spectrum, gain profile, and recovery time. This model 
was later on extended with the plasma effect [38] to account for the effective refractive index change and hence the phase shift term in order to provide for a comparative study of the performance characteristics of XGM- and XPM-based circuit topologies, indicating that the $\mathrm{XPM}$ phenomena are more resilient to high-speed operation.

As adopting explicit schemes for solving the system of coupled differential equations can become sometimes quite large even for a single SOA device, efficiency also needs to be addressed. Especially as integration technology and circuits evolve into complex networks, simulating multiple SOA devices simultaneously in the time domain in complex circuit configurations or with multi-wavelength signals travelling in various directions further exacerbates the numerical requirements. Simplified analytical gain models provide only qualitative conceptual results by suggesting exploring computational efficiency at the cost of accuracy [36]. In this regime, a few SOA models have been recently presented, demonstrating techniques to speed up the simulation time at the cost of some little accuracy. For instance, the use of one auxiliary signal with an effect equivalent to the amplified spontaneous emissions (ASE) was presented by Vujicic et al. [32] in order to reduce the wavelength channels under study. Almost similarly, a single-state variable depending on the available carrier densities within the SOA, termed as a "reservoir" of excited carriers, was presented by Mathlouthi et al. [33], resembling the reservoir of excited erbium ions in an erbium-doped fiber amplifier (EDFA). Despite the state variable, the model still relies on an equidistant spatiotemporal grid with constant time sampling at the output of the SOA, even at relatively low bitrates of $1 \mathrm{~Gb} / \mathrm{s}$.

\section{Accurate Transfer Matrix Method (TMM) SOA model}

The TMM numerical analysis technique was initially presented by Davis et al. [39] for DFB lasers and was initially presented for the extraction of the complete transfer matrix (TM) of large structures with rather simple configuration. The TMM divides the SOA longitudinally into $m$ small sectors of length $\Delta z=L / m$, where $L$ is the SOA length along the propagation direction. Considering a short sector length $\Delta z$ and a short time interval $\Delta t$, the light requires only a small time interval $\Delta t=u_{\mathrm{g}} \bullet \Delta z$, where $u_{\mathrm{g}}$ is the group velocity, to propagate through each sector. During this small time interval, the structural and material parameters of each sector, such as the carrier density and the material gain, can be considered uniform and constant, while transverse variations are still not allowed. This allows considering a wideband steady-state gain model.

The light propagation through a single sector can then be described by the simple TM, which relates the incoming and outgoing amplitudes of an elementary waveguide sector. For the current analysis and without loss of generality, we assume a signal Es propagating to the right direction and a second signal counterpropagating toward the left side, Ec. By applying the TM of sector $i$ at time $t$ for both the externally injected lights and the spontaneously emitted photons over a wide optical spectrum of 1500-1600 nm, we obtain the following equation: 


$$
\begin{gathered}
{\left[\begin{array}{c}
E s(t+\Delta t, i+1, \lambda i) \\
E c(t+\Delta t, i, \lambda i
\end{array}\right]=T M_{i} \times\left[\begin{array}{c}
E s(t, i, \lambda i) \\
E c(t, i+1, \lambda i
\end{array}\right]=} \\
=\left[\begin{array}{cc}
e^{\gamma(\lambda i) \Delta z} & 0 \\
0 & e^{\gamma(\lambda i) \Delta z}
\end{array}\right] \times\left[\begin{array}{c}
E s(t, i, \lambda i) \\
E c(t, i+1, \lambda i
\end{array}\right]
\end{gathered}
$$

where the propagation constant $\gamma(\lambda i)=g_{\text {eff }} / 2+j \bullet n_{\text {eff }} \omega / c$ describes the amplification (real part) and the phase shift (imaginary part) of the wave amplitude, after propagating through an elementary sector waveguide, as illustrated in Figure 1. Each updated wave amplitude that emerges at the output facet of a single sector can in turn be regarded as incident to the next sector, and the process is repeated until both lights exit the SOA. At each iteration, the waveguide propagation constant is updated and a new operant TM is estimated. This operation implies that a TM of a sector is time dependent since the matrix elements $e^{\gamma(\lambda i) \Delta z}$ are subject to the net gain coefficient $g_{\text {eff }}$ and phase shift changes $n_{\text {eff }}$. Figure 1(i) depicts the light propagation across the SOA lengths according to the TMM, while Figure 1(ii) depicts the $i$ th sector and the operation of the TM on the incoming and outgoing wave amplitudes.

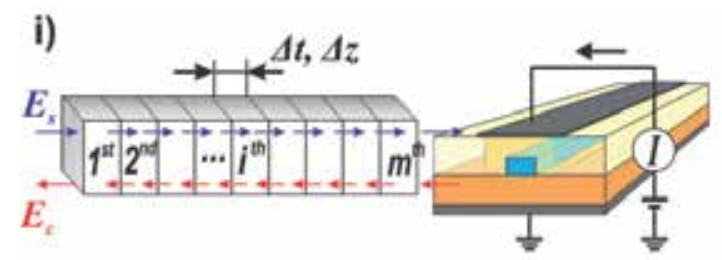

ii)

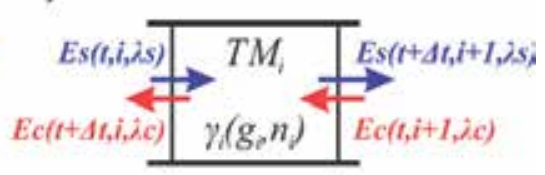

Figure 1. (i) Longitudinal division of an SOA into m sectors of $\Delta t$ and $\Delta \mathrm{z}$ time and space interval. (ii) Forward and backward light propagation and amplification through an elementary waveguide.

For the estimation of the real part of the constant, the Connelly gain coefficient has been employed, which was initially presented by Connelly [31] for the estimation of a wideband steady-state gain approximation of a bulk InP-InGaAsP homogeneous buried ridge stripe SOA, supporting large operating regimes. The gain coefficient is given by

$$
g_{m}=\frac{c^{2}}{4 \sqrt{2} \pi^{\frac{3}{2}} n a^{2} \tau v^{2}}\left(\frac{2 m_{e} m_{h h}}{\left(m_{e}+m_{h h}\right)}\right)^{\frac{3}{2}} \sqrt{v-\frac{E_{g}}{h}}\left[f_{c}(v)-f_{v}(v)\right]
$$

where $c$ is the speed of light in vacuum, $n_{\mathrm{a}}$ is the refractive index in the active region, $\tau$ is the radiative recombination lifetime, $v$ is the optical frequency, $m_{e}$ and $m_{h h}$ are the conduction band electron and valence band heavy hole effective masses, respectively, $\hbar$ is the Planck's constant divided by $2 \pi, E_{\mathrm{g}}$ is the bandgap energy and $f_{\mathrm{c}}(v)$, and $f_{\mathrm{v}}(v)$ is the Fermi-Dirac distributions in the conduction and valence band, respectively. Subsequently, we incorporate a loss coefficient along the SOA, toward a net gain coefficient given by [40] 


$$
g_{\text {net }}=\left(g_{\text {eff }}-a_{\alpha}\right) \Gamma-a_{\mathrm{c}}(1-\Gamma)-a_{\mathrm{s}}=g_{\mathrm{eff}} \Gamma-a_{\mathrm{WG}}
$$

where $\alpha_{\alpha}$ describes the losses in the active region, $\alpha_{\mathrm{c}}$ the losses in the cladding layer, and $\alpha_{\mathrm{s}}$ the scattering losses $m$ and by $\alpha_{\mathrm{WG}}$; we denote all cumulative losses. Carrier-dependent losses have also been presented in the literature, e.g., as given by $\alpha(N)-K_{0}+\Gamma K_{1} N$, with $K_{0}$ accounting for the overall material and waveguide losses of Eq. (3) and the second term accounting for the intervalence band absorption, which is current dependent. However, the constant term has also been shown to provide very good experimental matching. After the estimation of the updated amplitude, amplified by the net gain coefficient through propagation by one sector, the power emerging at the output facet is given by [39]

$$
P(i, t)=h v w d|E(i, t)|^{2}
$$

where $h v$ is the photon energy, $w$ is the width, and $d$ is the thickness of the active region of the SOA, while the photon density of each stream is approximated by [39]

$$
S(i, t)=\frac{|E(i, t)|^{2}}{u_{g}}
$$

Finally, more rigorous electron statistics and fast nonlinear phenomena of the spectral hole burning and the intraband phenomena are only taken into consideration through the use of the gain compression factor $\varepsilon$, as governed by [40]

$$
g_{\text {eff }}=\frac{g_{\text {net }}}{1+\varepsilon S}
$$

Following the estimation of the photonic densities for all signals travelling through each sector, we update the carrier density $\mathrm{N}_{\mathrm{i}}$ according to the well-known rate equation $[39,40]$ :

$$
\frac{\partial N_{i}}{\partial t}=\frac{J}{e d}-N_{i}\left(c_{1}+c_{2} N_{i}+c_{3} N_{i}^{2}\right)-\sum_{u=1,2,3} u_{\mathrm{g}} \Gamma g_{\mathrm{m}}^{(u, i)} S_{i}
$$

where $J$ is the injection carrier density; $c_{1}, c_{2}$, and $c_{3}$ are the current leakage, radiative recombination, and Auger recombination rates; $\Gamma$ is the confinement factor; and $S_{i}$ is the photon density at the $i$ th sector. The index $u=1,2,3$ refers to the ASE or the two input streams.

Having updated the carrier density, we can proceed to the estimation of the imaginary part of the propagation constant, which accounts for the phase shifting term of the amplitude. The 
estimation of the effective refractive index $n_{\text {eff }}$ in the imaginary part relies on the plasma effect, according to which the change of the refractive index $\Delta n_{p l}$ is linearly dependent on the change of the free carrier density in the active region $\Delta N[40]$ :

$$
\Delta n_{\mathrm{pl}}=-\frac{e^{2} \Delta \mathrm{N}}{2 \omega^{2} \varepsilon_{o} n_{\mathrm{eq}}}\left(\frac{1}{m_{\mathrm{e}}}+\frac{1}{m_{\mathrm{hh}}}\right)
$$

where $e$ is the electronic charge, $\omega$ is the angular frequency, $\varepsilon_{0}$ is the permittivity of free space, $n_{\mathrm{eq}}$ is the effective index of the waveguide, and $m_{\mathrm{e}}$ and $m_{\mathrm{hh}}$ are the effective mass of the electron and the heavy hole. An accurate estimation of the real and imaginary part of the propagation constant $\gamma(\lambda i)=g_{\text {eff }} / 2+j \bullet n_{\text {eff }} \omega / c$ of the $i$ th sector allows describing the forward and backward (counter)propagating amplitudes through a simple time dependent TM given in Eq. (1).

\section{Gain parameterization procedure}

In this section, we report on a gain parameterization procedure and a methodology toward tailoring the TMM model and producing simulation results close to the experimental measurements obtained from a commercial SOA device. The methodology is in principle generic and compatible with the other SOA devices. The target is to identify a set of material parameters and cross-sectional characteristics by incorporating feedback from experimental measurements such as the bandgap shrinkage to fit the experimental gain spectrum in a spectrum analyzer, the recombination rates to match the gain profile of gain-vs-incoming power measurements, and the gain compression factor for the recovery time at pump probe.

The procedure includes a number of steps clustered into two sections: first, the definition of the material and waveguide properties and, second, the feedback from the experimental characterization of the SOA device under study.

The material and waveguide properties include the following:

1. To define the length of the active region of the fabricated SOA waveguide, according to foundry.

2. To estimate of the initial bandgap energy $\mathrm{Eg} 0$ of the active region, assuming no external current injection

3. To define the loss coefficient of the waveguide

4. To incorporate the waveguide cross-sectional dimensions, e.g., core height and width

5. To define the external operating current I and the current density injected to the active region.

The cross-sectional dimensions and the length of the SOA waveguide or the active material can be provided by the fabrication foundry. For the definition of the material properties, 
extensive literature [45-48] has been developed on III/V compounds, especially for common compounds such as the InGaAsP-based devices. The initial bandgap energy $E_{\mathrm{g} 0}$ when no carriers are injected in the active region can be provided by the quadratic approximation $E_{\mathrm{g} 0}=e\left(a+b y+c y^{2}\right)$ [31], with $e$ being the electronic charge and $y$ the molar fraction of the Arsenide in the active region, and $a, b$, and $c$ being the coefficient of the quadratic approximation. Similarly, the comprehensive model presented by Cheung et al. [48] and dealing with the optimization of the structural and material parameters of $\operatorname{In}(1-x-y) \mathrm{Ga}(x) \mathrm{Al}(y)$ As quantumwell (QW) SOAs toward bonding on an SOI platform provide an in-depth insight into bandgap energy.

In addition to the definition of the material properties, feedback from a series of experimental characterization measurements on real SOA devices can be incorporated in the model through the following three steps:

6. To define the bandgap shrinkage coefficient to fit the experimental gain spectrum of the SOA. The bandgap shrinkage coefficient $\mathrm{Kg}$ affects the blue shifting of the gain peak at the ASE output spectrum of the SOA, which occurs when increasing the injection current. An increase in the carrier density is translated into shrinkage of the material bandgap by a term $\Delta E_{\mathrm{g}}=e K_{\mathrm{g}} N^{1 / 3}$ [31]. In order to extract the parameter for the $\mathrm{Kg}$ coefficient, static measurements of the ASE spectrum at the output of the SOA under different external current injection and no injected optical power are suggested.

7. To define the recombination rates for the gain curve versus the incoming optical power. This includes the $c 1, c 2$, and c3 parameters that are associated with the linear recombination coefficient at defects (current leakage), the spontaneous recombination, and the Auger recombination rate, respectively. In practice, these three mechanisms exhibit complicated dependences that together account for the overall recombination rate given by $R(N)=c_{1}+c_{2} N^{2}+c_{3} N^{3}[31]$. This step requires static power gain measurements using a continuous wavelength light source close to the gain peak wavelength and under varying average optical power in order to estimate net gain of the SOA, and thus defining the small signal gain and the saturation point of the SOA.

8. To define the gain compression factor to fit the recovery time. The gain dynamics associated with the intraband phenomena are associated with a fast recovery time immediately after the injection of a short pulse, with the gain compression increased under increasing pulse energy and bias current. Although the TMM model focuses on the interband phenomena, the ultrafast intraband phenomena have been considered through the use of a power-dependent gain compression coefficient [40], which allows tuning the fast recovery time of the intraband phenomena. In this strep, typical pump probe measurements are required with a weak Continuous Wavelength signal with average optical power in the small signal gain fed to an SOA along with a high-power ultrashort return-to-zero pulse with a full width half maximum duration of 5 ps, which sweeps all the available carriers. 
Considering the numerous interrelated phenomena that affect the performance of an SOA, determining the optimum parameters that match all system-level parameters, such as the gainpower curve, the spectrum, and the recovery time, implies a certain difficulty. Defining a certain methodology for parameter extraction ensures accuracy of the model for direct applicability in realistic use case scenarios and demonstrations. After developing a certain gain parameterization procedure, the use of multiobjective genetic algorithms supporting an automated iterative procedure can also been adopted [32] toward a best-fit criterion.

\subsection{Quantitative experimentally verified numerical measurements}

Following an experimental characterization on a commercial SOA device [50], the previous gain parameterization procedure was adopted in order to extract the optimum simulation parameters and tailor the TMM model. Figure 2(i) illustrates the experimental setup used for the characterization of the SOA device. Two tunable laser diodes (TLD) operating at wavelengths $\lambda 1$ and $\lambda 2$ were employed characterization of the SOA. The CW output at $\lambda 1$ of the first TLD was amplified through an EDFA and filtered through a band-pass filter (BPF) before being driven to the input of the SOA. The CW output at $\lambda 2$ of the second TLD was modulated by means of an electroabsorption modulator (EAM), whose operation was driven by an electrical clock signal. In this way, short optical return-to-zero (RZ) pulses were produced amplified through an EDFA and filtered through a BPF at $\lambda 2$ wavelength. The produced RZ pulse stream was coupled with the $\lambda 1 \mathrm{CW}$ and also fed to the SOA. Polarization controllers (PCs) were employed at various stages of the experimental setup to adjust the polarization of the optical signals as discrete off-the-shelf fiber pigtailed components were employed. Using the presented experimental setup, all three measurements of steps 6, 7, and 8 were conducted using variable optical attenuators (VOAs) at the fiber-pigtailed branches before the SOA. VOAs allowed controlling the average power of the optical signals properly. In step 6, no optical input fed to the SOA, and both VOAs provided maximum attenuation, while the SOA output was recorded at an optical spectrum analyzer (OSA). By measuring a peak gain around 1560 $\mathrm{nm}$, at step 7, the CW signal of TLD1 was tuned at $\lambda 1=1555 \mathrm{~nm}$, and the output of the SOA was measured by a power meter, after filtering through a BPF.

The TMM model was in turn tailored to match the experimental measurements. Figure 2(ii) illustrates the normalized simulated optical field distribution along the SOA using the TMM, with the vertical $z$-axis being the optical power and the horizontal $x$-axis being the wavelength range between 1520 and $1580 \mathrm{~nm}$. The plot illustrates how an input simulation signal at 1555 $\mathrm{nm}$ wavelength of the SOA is propagated together with the generated ASE noise sector by sector, as marked in the $y$-horizontal axis, until it emerges at the final sector at the SOA output. Moreover, Figure 2(iii)-(v) demonstrates the simulation-experimental matching: The gain versus input power in Figure 2(iii) for a single $\lambda 1-\mathrm{CW}$ signal reveals accurate matching both in the small signal region as well as in the saturate gain regime, with less than $1 \mathrm{~dB}$ error for an input power between -30 and $0 \mathrm{dBm}$. Additionally, the simulated output spectrum fits well the experimentally recorder spectrum with less than $2 \mathrm{~dB}$ errors, while the matching in the time domain of the $10 \%-90 \%$ gain-recovery time of the SOA reveals accurate prediction of the response of the SOA in the ps time scale. The simulation parameters extracted by this procedure are presented in Table 1. 


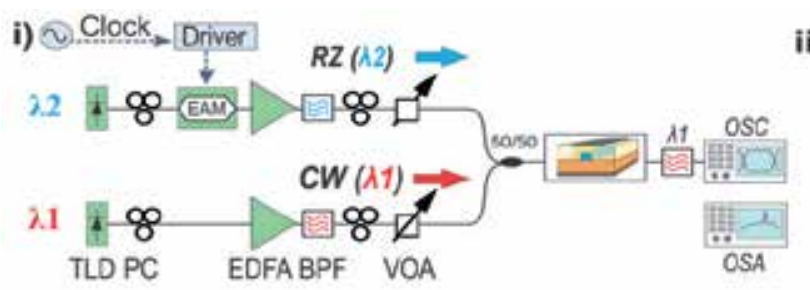

ii)
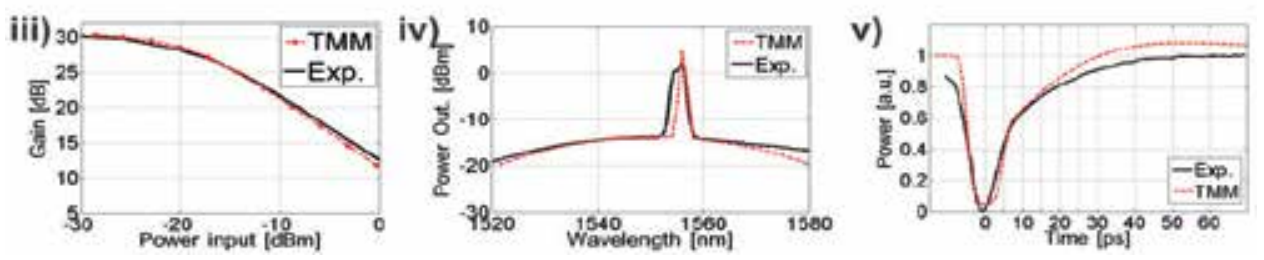

Figure 2. (i) Experimental setup employed for the characterization of the SOA device for feedback to the TMM simulation model. (ii) Propagation of the signal across 15 SOA sectors according to the TMM model in the 1520-1580 range and simulation-experimental matching for (iii) the gain versus the input optical power, (iv) the output spectrum with $30 \mu \mathrm{W} C W$ input, and (v) the recovery time of after pump-probe measurements

\begin{tabular}{lll}
\hline Symbol & Parameter description & Value \\
\hline$m$ & Number of longitudinal & 20 \\
\hline$\Delta t$ & Temporal sector interval & $1[\mathrm{ps}]$ \\
\hline$L$ & SOA length & $1600[\mu \mathrm{m}]$ \\
\hline$w$ & Active layer width & $1.2[\mu \mathrm{m}]$ \\
\hline$\Gamma$ & Active layer thickness & $0.1[\mu \mathrm{m}]$ \\
\hline$u_{\mathrm{g}}$ & Confinement factor & 0.17 \\
\hline$c_{1}$ & Group velocity & $8.510^{8}[\mathrm{~m} / \mathrm{s}]$ \\
\hline$c_{2}$ & Linear recombination rate & $110^{7}[\mathrm{~m} / \mathrm{s}]$ \\
\hline$c_{3}$ & Bimolecular recombination rate & $5010^{-17}\left[\mathrm{~m}^{3} / \mathrm{s}\right]$ \\
\hline$a_{\mathrm{wg}}$ & Auger recombination rate & $8010^{-41}\left[\mathrm{~m}^{6} / \mathrm{s}\right]$ \\
\hline$R$ & Waveguide losses & $5800[1 / \mathrm{m}]$ \\
\hline$I$ & Reflectivity at facets & 0 \\
\hline$n_{\mathrm{ao}}$ & External current injection & $300[\mathrm{~mA}]$ \\
\hline$n_{c l}$ & Reflective index in the active region & 3.22 \\
\hline$E_{g}$ & Refractive index in the cladding & 3.1 \\
\hline
\end{tabular}

Table 1. Main parameters of the SOA device used in the simulation

\subsection{Qualitative results on SOA gain recovery time}

To accommodate the increasing demand of data transfer and high-speed optical telecommunication networks with terabit transmission capabilities and high bandwidth switching functionalities, there has been a growing interest in increasing the recovery time of the SOA. High-speed nonlinear SOAs are used to perform either XGM or XPM modulation between two 
input signals, i.e., a weak CW probe signal and a high-speed data signal with a short pulse width, acting as pump. Increasing the external current injection or the elongating the SOA active region have been shown to shorten the recovery time and thus support higher speed switching operations. After developing an experimentally verified time domain model, we numerically investigate the shortening of the recovery time during pump probe measurements under increasing current or increasing SOA length.

Figure 3(i) illustrates the simulated gain recovery and the normalized output power of the SOA for various external currents. The external currents were tuned from 185 to $350 \mathrm{~mA}$, with the $300 \mathrm{~mA}$ being the nominal current operation. The plots reveal a shortening at higher currents, stemming from the increased current density injected to the SOA. The measured 1/e gain recovery times were plotted versus the supplied current in the inset, demonstrating a shortening from 45 ps under $185 \mathrm{~mA}$ down to 20 ps under $350 \mathrm{~mA}$.

Similarly, Figure 3(ii) illustrates the simulation results for the normalized SOA power output and the gain recovery after pump probe measurements, while the external current injection was maintained constant at nominal values of $300 \mathrm{~mA}$. The plots reveal a shortening of the recovery time when elongating the SOA from 0.5 to $1.7 \mathrm{~mm}$. The obtained $1 / e$ recovery time was measured and plotted in the inset, indicating a shortening from 63 ps for a 0.5 -mm-long SOA down to 20 ps for a $1.7-\mathrm{mm}$-long SOA.
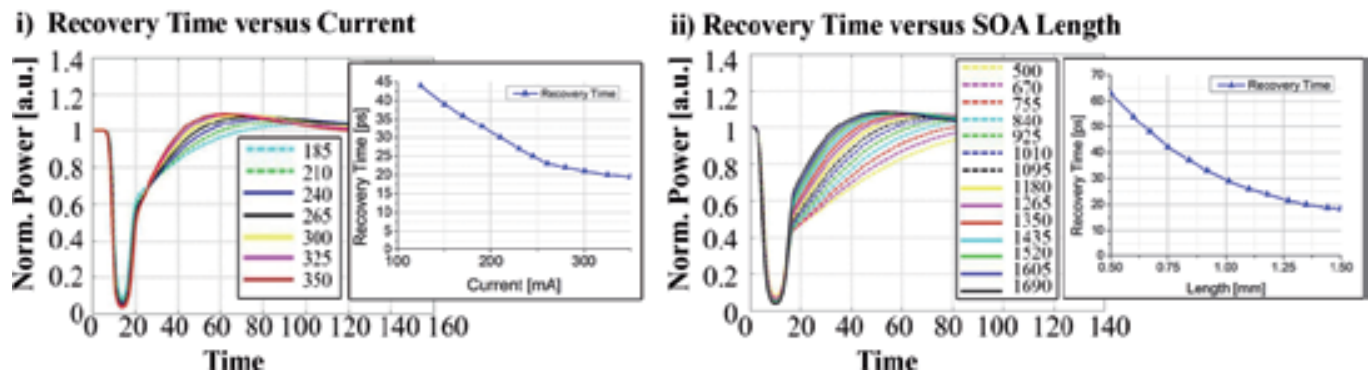

Figure 3. (i) Gain recovery of the SOA for increasing external current injection ranging from 185 to $350 \mathrm{~mA}$. The inset plots the measured 1/e recovery time versus the current. (ii) Gain recovery time of the SOA for different lengths of the active region ranging between 500 and $1700 \mu \mathrm{m}$. The inset plots the measured 1/e recovery time versus the SOA length.

The effect of the propagation direction of the pump signal was also investigated using the TMM SOA model. Figure 4 depicts the simulated time traces of the normalized output power and the phase shift of the probe light, obtained after pump probe measurements with the pump control pulse being fed in a co- or counterpropagating direction. The $\mathrm{CW}$ probe signal featured an average power of $50 \mu \mathrm{W}$, which lies in the small signal regime, while the peak of the control pump pulse was tuned, so as to induce a $\pi$ phase shift at the CW signal for either propagation direction.

The normalized traces are plotted of the XPM operation imprinted on the probe signal are illustrated in Figure 4(i) and the XGM operation in Figure 4(ii). It is obvious that in both cases, a $\pi$ phase shift was introduced due to XPM operation. However, the probe signal is suppressed 
more in the case of the copropagating pump pulse, reaching normalized power values down to 0.1 , while for the counterpropagating pump pulse the gain suppression is less deep. This owes to the increase pump-probe light interaction along the copropagating direction through the carrier rate equation. In addition, to the recovery time in case of a counterpropagating control pulse is shorter (after the suppression of the gain a local minima), however, the tradeoff is a slower response/fall time observed with a delay in the deep (minima of the output power) when the control pulse is injected in the counterpropagating direction. This owes again to the travelling time and the time required for the counterpropagating pump pulse to absorb the available carriers. The findings for XGM effects and XPM effects for $\pi$-phase shift of a Gaussian control pulse are shown in Figure 4 and are in full compliance with the ones found in the literature [41].

\section{i) Cross Phase Modulation of $\pi$}

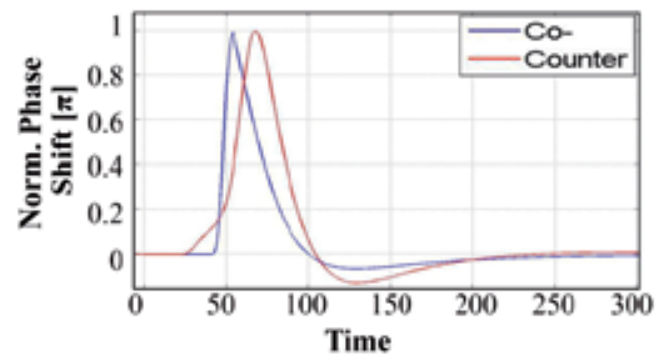

ii) Cross Gain Modulation

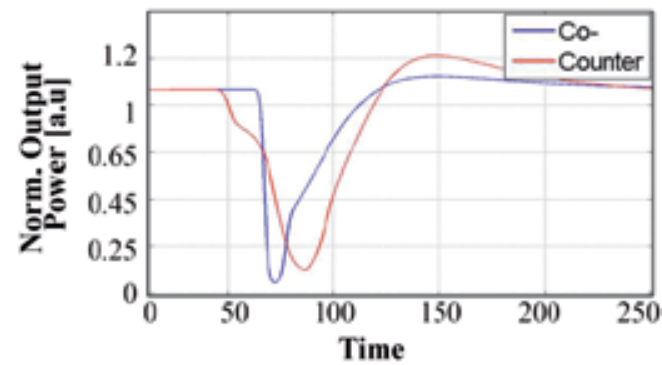

Figure 4. (a) XGM and (b) XPM effects imprinted on a CW probe signal by a co- or counterpropagating RZ pump pulse that induces a $\pi$ phase shift.

\section{Efficient multigrid SOA model with adaptive time stepping (TS)}

Numerical SOA modeling has so far relied on explicit schemes for solving the associated system of coupled ordinary differential equations (ODE), comprising the spatial discretized carrier density rate equation given in Eq. (6) combined with the material gain coefficient in Eq. (2). In the literature, ODEs are classified in two categories: the stiff and nonstiff ODEs. The latter can be solved efficiently by explicit time stepping schemes. The former requires many steps with explicit schemes as warranted by the smoothness of the solution, as has been the case for the traditional SOA modeling and is schematically illustrated in Figure 2(ii) for the TMM model, where each node of the plot represents a small time step. An interesting alternative option would be to deploy implicit schemes, which could alleviate the problem of the many unneeded, as far as accuracy is concerned, time steps at the cost of having to determine the Jacobian for the set of ODEs and inverting a matrix, at each time step. Implicit schemes have shown to be more efficient for many problems [42], with multigrid methods known to be among the most efficient solvers for many partial differential equations (PDEs) [43]. 
Vagionas and Bos [44] presented a novel multigrid solver for the dynamic response of the SOA, relying on the wideband steady-state gain coefficient of Eq. (2). Introducing multigrid techniques in SOA modeling enabled extending the accurate time domain modeling of the TMM model, allowing for the development of an efficient solution supporting implicit time discretization schemes. Implicit schemes in turn enable accuracy-instead of stabilityrestricted time discretization of the signals. This implies a different discretization scheme, where sampling of the signals is optimized for certain accuracy in the solver instead of certain time step restriction. This allows lifting the limitations of an equidistant spatiotemporal grid for the representation of the incoming signals adopted by traditional explicit SOA models, releasing an adaptive step size-controlled solver for the dynamic SOA response with dense time sampling under a rapidly varying SOA signal output and scarce time sampling when negligible changes are observed. Adaptive times stepping adds one more degree of freedom to computational efficiency of accurate SOA modeling, which is of crucial importance when evaluating large input patterns for statistical signal analysis independent of the bitrate or large circuit networks with multiple SOA-based components.

Multigrid methods employ a series of coarser grids to obtain grid independent convergence rates. Drawing from the finest grid of traditional longitudinal division of the SOA into cascaded sectors, coarse and coarser grids with less number of SOA sectors are adopted in order to represent the spatial free carrier density distribution by less and less grid points (carrier density samples). This is schematically illustrated in Figure 5(i), where 4 grid levels have been employed. The 4 th level is the finest level, including an SOA longitudinal discretization into 16 sectors, equal to the discretization employed in the TMM model. However, by applying multigrid concepts, the carrier densities of two neighboring sectors can be represented by a single sector in the coarser 3rd-level grid, resulting in half grid points. Equivalently, the 3rd-level can be again restricted to coarser grids, with each transition halving the number of grid points.
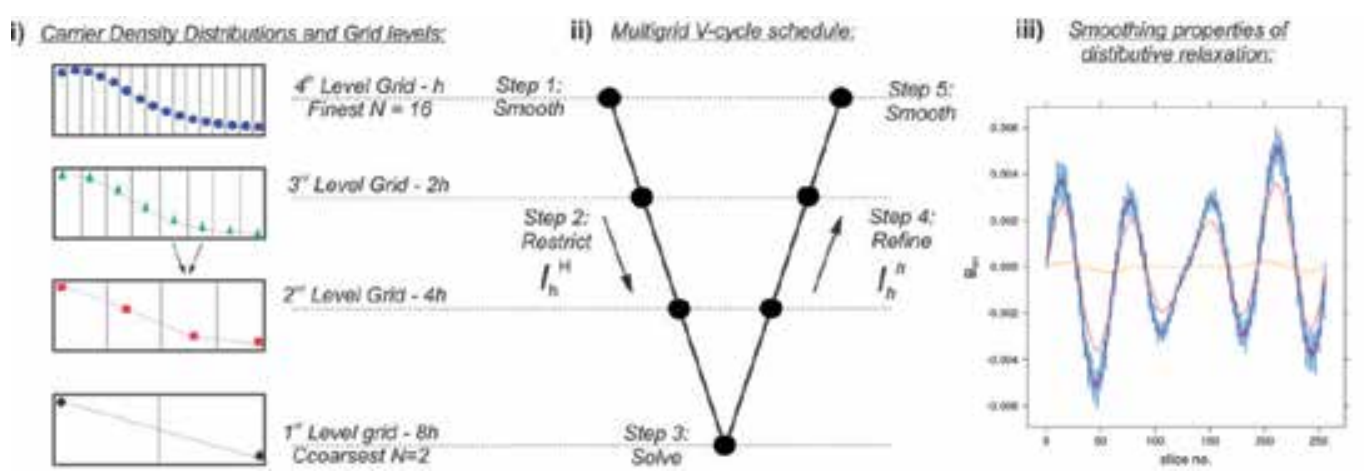

Figure 5. (i) A series of four grids illustrating the finest SOA carrier density granularity of $N=16$ sectors down to the coarsest grid of $N=2$ sectors, which represent the carrier density distributions along the SOA. (ii) Graphic representation of the five step multigrid V-cycle schedule. The initial finest approximation is smoothed (step 1), restricted to the coarsest grid (step 2), solved with coarse granularity (step 3), refined to the finest grid (step 4), and finally smoothed again (step 5). 
In the following the notation, $h=\Delta \mathrm{z}$ and $\mathrm{H}=2 \Delta \mathrm{z}$ are used for the representation of the SOA sector length in the fine and coarse grid, respectively. In order to focus on the multigrid aspects, the rate equation of Eq. (6) is written in an operator form as follows:

$$
\frac{\partial N_{i}^{h}}{\partial t}=L^{h} N_{i}^{h}+f_{i}^{h}
$$

where the operator $L$, although simple in appearance, is the quite complex, as it involves solving the forward and backward propagation equations for both the signals and noise photon fluxes. By applying the implicit midpoint rule, we obtain the following equation:

$$
L^{h, k} N_{i}^{h, k}=\frac{N_{i}^{h, k}-N_{i}^{h, k-1}}{\Delta t_{k}}-L^{h} \frac{N_{i}^{h, k}+N_{i}^{h, k-1}}{2}=f_{i}^{h, k}
$$

where $t_{\kappa}=t_{\kappa-1}+\Delta t_{k}$ and $f_{i}^{h, k}=0$ for $h=\Delta z$. The transition from a fine toward coarser grid can be achieved through an averaging restrict operator $I_{h}{ }^{H}$, where a coarse grid point can be considered as an average of two neighboring fine points. Equivalently, the operator $I_{H}{ }^{h}$, $I_{h}^{H}$, and $I_{H}^{h}$ refines the coarse grid functions to a finer grid. In order to generate an intermediate fine point between two coarse grid points, an inverse operator has to be considered compared to the restrict operation, which in our case is the linear approximation. In this way, a multigrid correction V-cycle $\operatorname{MG}\left(\hat{N}^{h, k}, L^{h, k}, f^{h, k}\right) \rightarrow \bar{N}^{h, k}$ has been developed, described by the following five steps:

$$
\begin{aligned}
& \text { Step1 }: \tilde{N}^{h, k} \leftarrow \operatorname{smooth}\left(\hat{N}^{h, k}, L^{h, k}, f^{h, k}, v_{1}\right) \\
& \text { Step2 }: \hat{N}^{h, k} \leftarrow I_{h}^{H}\left(\hat{N}^{h, k}\right), f^{H, k}=L^{H, k}\left(I_{h}^{H} \tilde{N}^{h, k}\right)+I_{h}^{H} r^{h, k} \\
& \text { Step3 }: \operatorname{solve}\left(\hat{N}^{H, k}, f^{H, k}\right) \rightarrow \tilde{N}^{h, k} \\
& \text { Step4: } \tilde{N}^{h, k}=\tilde{N}^{h, k}+I_{H}^{h}\left(\hat{N}^{H, k}-I_{h}^{H} \tilde{N}^{h, k}\right) \\
& \text { Step5 }: \bar{N}^{h, k} \leftarrow \operatorname{smooth}\left(\tilde{N}^{h, k}, L^{h, k}, f^{h, k}, v_{2}\right)
\end{aligned}
$$

The developed multigrid V-cycle, including the above five steps, is schematically represented in Figure 5(ii), showing four grids of different granularity. Each coarse grid comprises half the grid points compared with the higher/finer grid level, while transitions rely on the $I_{h}^{H}$ restrict and $I_{H}{ }^{h}$ refine operators. The smooth operation exist of second-order distributive Jacobi relaxations has been considered, that is, for each, $N_{t}{ }^{h, \kappa}$, an update is calculated as follows:

$$
\delta_{i}^{h, k}=\left(f_{i}^{h, k}-L^{h, k} N_{i}^{h, k}\right) /\left(\frac{1}{2} \frac{d L_{i}^{h, k}}{d N_{i-1}^{h, k}}-\frac{d L_{i}^{h, k}}{d N_{i}^{h, k}}+\frac{1}{2} \frac{d L_{i}^{h, k}}{d N_{i+1}^{h, k}}\right)
$$


The solve operation can be implemented recursively, while the solution of the coarse grid problem is used to correct the fine grid approximation. High-frequency components owing to the interpolation of the correction cycle can be removed by the final smooth operations. The presented cycle features a grid-size-independent rate of reducing in the error by $e^{h, k}=\hat{N}^{h, k}+N^{h, k} \mathrm{e}^{\mathrm{h}, \mathrm{k}}=\left\|\hat{N}^{\mathrm{h}, \mathrm{k}}-\mathrm{N}^{\mathrm{h}, \mathrm{k}}\right\|$. When using the solution of the previous time step as initial approximation to the solution at the current time step, $k, O(\log (1 / h))$ cycles are needed to solve the problem to the level of the truncation error. This can be reduced to $O(1)$ by using the coarser levels to generate an initial approximation accurate to within the level of truncation of grid, $H$ [43].

The proposed solver employs the rate equation (Eq. 6) and the multigrid techniques to solve the carrier density distribution along the SOA in coarser and coarser grids, while the propagation and amplification of the signal is still based on the Connelly material gain coefficient employed in the TMM model [37]. Adopting the previous gain coefficient ensures equivalent steady-state results, such as the optical spectrum and the net gain, and tailoring of the SOA parameters with experimental measurements. On the other hand, incorporating an implicit time discretization scheme and adaptive time sampling suggests that computational efficiency is exploited based on the adaptive time sampling in order to benefit from long bit patterns or small pattern changes at the input bit-streams.

The increase or decrease of the time step is controlled by the implicit midpoint rule with a doubling scheme through the accuracy tolerance parameter $\varepsilon$, as depicted in Figure 6 for various accuracy tolerances of the multigrid solver and single block pulses at different bitrates. The simulation results depict the pulsed NRZ transmission through a single SOA with single pulses of $-25 \mathrm{dBm}$ peak power centered at $1550 \mathrm{~nm}$ for a bitrate of $1 \mathrm{~Gb} / \mathrm{s}$ in Figure 6(i) and 5 $\mathrm{Gb} / \mathrm{s}$ in Figure 6(ii). The accuracy tolerance ranges between 1e-2 for the plot at the top right corner of the graphs and $1 \mathrm{e}-5$ for the bottom left plot, highlighting that a dense time sampling is adopted immediately at the rise and fall time of the pulse, where transient phenomena are observed. On the contrary, the trailing part of a bit pulse before the bit transitions of the output pattern, a steady state is obtained in carrier density distribution resulting in steady-state SOA gain dynamics. Thus, negligible changes are observed at the output of the SOA, revealing than the time step can be adapted to a larger value for enhanced computational efficiency.

The multigrid solver was also employed toward simulating the XGM operation between two signal in pump probe measurements. A pump control bit stream is wavelength converted on a $\mathrm{CW}$ probe signal at $10 \mathrm{~Gb} / \mathrm{s}$ bitrate, with the $\mathrm{CW}$ featuring an average power of $-25 \mathrm{dBm}$ at $1550 \mathrm{~nm}$ and the NRZ bit stream exhibiting a peak power of- $25 \mathrm{dBm}$ at $1555 \mathrm{~nm}$. A custom control bit pattern of 001100 at $10 \mathrm{~Gb} / \mathrm{s}$ was employed, as this pattern changes at odd bits (1st, 3rd, and 5th bit) with fast changes in the carrier dynamics necessitating dense time sampling. On the contrary, during the even bits, when no changes are observed at the logical values of the input data streams, the response of the SOA is characterized by a constant steady-state response and thus requires only a few time samples. This is characteristically illustrated in Figure 7(i), where the even bits necessitate only very few samples, as highlighted with a yellow marker at the cost of a user-defined accuracy error. Adaptive time stepping can be important 
i) $1 \mathrm{~Gb} / \mathrm{s}$ operation

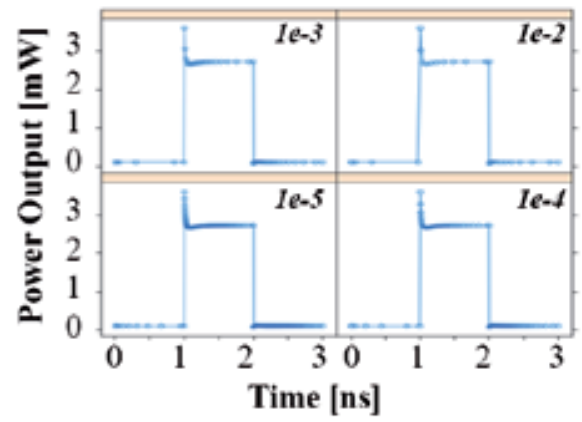

ii) $5 \mathrm{~Gb} / \mathrm{s}$ operation

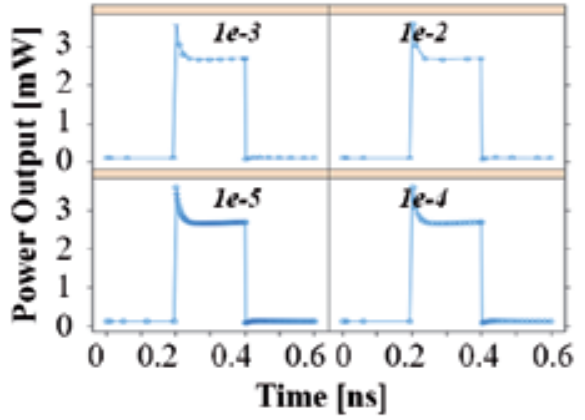

Figure 6. Simulation results for a block pulse of $-25 \mathrm{dBm}$ peak power at $1550 \mathrm{~nm}$ wavelength input propagating along the SOA, illustrating the adaptive time sampling at the output of the SOA with dense time sampling after the bit transition from 0 to 1 and from 1 to 0 and scarce time sampling at the end of the bit pulse, where a constant SOA steadystate implies negligible SOA outputs. The results have been obtained with an accuracy tolerance of $\varepsilon=1 \mathrm{e}-2$ (top right corner), $\varepsilon=1 \mathrm{e}-3$ (top left), $\varepsilon=1 \mathrm{e}-4$ (bottom right), and $\varepsilon=1 \mathrm{e}-5$ (bottom left) for bitrates of (i) $1 \mathrm{~Gb} / \mathrm{s}$ and (ii) $5 \mathrm{~Gb} / \mathrm{s}$.

for statistical performance evaluation of SOA-based circuits, especially when long patterns or complex structures are employed.

\section{i) Adaptive Time Stepping}

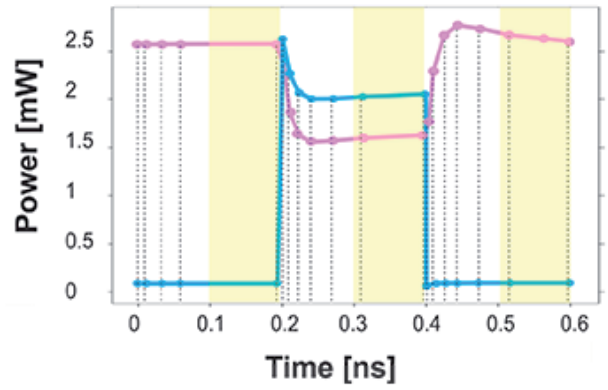

ii) Average samples/bit

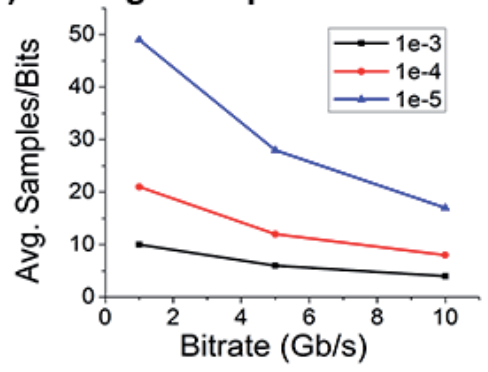

Figure 7. (i) Cross-gain modulation between a CW input signal of- $25 \mathrm{dBm}$ peak power at $1550 \mathrm{~nm}$ and an NRZ stream with a peak power of $-25 \mathrm{dBm}$ at $1555 \mathrm{~nm}$ using the adaptive multigrid solver at $10 \mathrm{~Gb} / \mathrm{s}$. The dotted lines mark the adaptive time-stepping mechanism of the multigrid solver (ii) plot of the average number of samples per bit required versus the bitrate operation.

In order to evaluate the performance and computational efficiency of the solver, the operation of wavelength conversion of a control pulsed signal to a CW input signal has been simulated at different bitrates and accuracy levels. The evaluation is based on the number of required samples that describe the output of the SOA, considering an NRZ control signal following a $2^{7}-1$ pseudo random bit sequence (PRBS) at bitrates of $1 \mathrm{~Gb} / \mathrm{s}, 5 \mathrm{~Gb} / \mathrm{s}$, and $10 \mathrm{~Gb} / \mathrm{s}$. The results have been summarized in Figure 7(ii), showing the average number of samples per bit required for the overall simulated pattern versus the bitrate. Three different accuracy errors have been considered, namely, 1e-3, 1e-4, and 1e-5. Especially in the case of relatively big tolerance of $\varepsilon$ 
$=1 \mathrm{e}-3$ in the first column, bits are resolved by 4 and 6 samples for the bitrates of $10 \mathrm{~Gb} / \mathrm{s}$ and $5 \mathrm{~Gb} / \mathrm{s}$, respectively. Furthermore, for bitrates of $1 \mathrm{~Gb} / \mathrm{s}$, an average of 10 samples per bit is required, suggesting that the 4 samples describe efficiently the first 100 ps (for $10 \mathrm{~Gb} / \mathrm{s}$ operation), additional two samples are required for the next 100 ps (for $5 \mathrm{~Gb} / \mathrm{s}$ ), while the rest of the four samples out of ten can effectively describe the trailing $800 \mathrm{ps}$ of the bit duration of $1 \mathrm{~Gb} / \mathrm{s}$.

\section{Circuit-level simulation and applications}

Using the developed SOA numeric modeling tools, we numerically evaluated two SOA-based circuits predominant in all-optical signal processing: (i) an all-optical flip-flop architecture that exploits coupled SOA waveguides, operating on XGM phenomena, and (ii) an all-optical XOR gate that exploits an SOA-MZI configuration and XPM phenomena. The numerical results are compared with the results experimentally obtained, showing very good agreement.

\subsection{Coupled SOA flip-flop exploiting XGM phenomena}

The proposed SR-Flip Flop relies on the bistability between a slightly and a fully saturated regime of two travelling waveguide SOA-XGM switches [37]. Each SOA is powered by a weak $\mathrm{CW}$ at $\lambda 1$ or $\lambda 2$ wavelength, respectively. Coupled together through a 70/30 coupler as shown in Figure 8(i), they form a simplified version of an SR-Flip Flop. The coupler defines the coupling efficiency between the two SOAs and is also used for inserting the set/reset pulses to the SOAs and driving the SR-Flip Flop states at the outputs. The 70/30 ratio was found to be the optimum for the trade-off between the SOA coupling efficiency and power ratio at the input/output ports. Exploiting XGM phenomena, one SOA at a time acts as master suppressing the other, which consequently acts as slave. Due to symmetrical setup, the role of master and slave can be interchanged and the state of the SR-Flip Flop is determined by the wavelength of the dominating output. A logical "1" corresponds to SOA1 being dominant and $\lambda 1$ suppressing the SOA2 output signal, whereas a logical " 0 " is obtained when SOA2 dominates the SR-Flip Flop. Switching between the two states requires injecting proper external set or reset pulses at the dominating SOA through the corresponding branches. When a bit of logical " 1 " set signal at $\lambda 1$ is injected into the dominating SOA1 of logical " 1, " its gain is saturated and the transmission of $\lambda 1 \mathrm{CW}$ input signal is blocked. This allows SOA2 gain to recover, unblocking the transmission of CW $\lambda 2$ and switching the SR-Flip Flop state to logical "0." The high $\lambda 2 \mathrm{CW}$ now is fed into SOA1 serving as the control signal that suppresses its gain even after the set pulse is extinct. As a result, SOA2 acts as master dominating over the slave SOA1, and the SR-Flip Flop will remain in this state until a reset pulse of $\lambda 2$ wavelength is fed into SOA2. This will saturate its gain and unblock the transmission of $\lambda 1 \mathrm{CW}$, switching the SR-Flip Flop back to its initial state of logical "1."

The flip-flop operation was verified numerically and experimentally with set and reset pulse traces, as shown in Figure 8(ii) and (iii), respectively. The simulated output of the flip-flop at $10 \mathrm{~Gb} / \mathrm{s}$ for both flip-flop outputs employed the same set/reset patterns as the experimentally 
employed, shown in Figure 8(iii). The experimental demonstration was performed at a lowoperation speed as dictated by the 8.5-m-long fiber-pigtailed coupling stage between the two SOAs, which seems to be the main speed-determining factor [38]. The results of Figure 8(iii) were recorded for $\lambda 1$ and $\lambda 2 \mathrm{CW}$ signals of $150 \mu \mathrm{W}$ before entering the SOAs and set/reset pulses of $1.8 \mathrm{~mW}$ prior to reaching the 50/50 couplers and the corresponding branches of the flip-flop. The two SOAs were driven at $300 \mathrm{~mA}$.

Although the experimental data results confirm successful SR-Flip Flop operation, exhibiting an average extinction ratio (ER) of $7 \mathrm{~dB}$ and an amplitude modulation (AM) of less than $2 \mathrm{~dB}$, due to the fiber pigtailed implementation and the large fiber lengths, the operation was limited at low operating speeds. In order to confirm that multi-Gb/s operation speed is possible, we have applied in the numerical evaluation of the proposed SR-Flip Flop the same pattern to the external signals, as the ones employed experimentally, but at $10 \mathrm{~Gb} / \mathrm{s}$. CW beams of $150 \mu \mathrm{W}$ at $1548 \mathrm{~nm}(\lambda 1)$ and $1550 \mathrm{~nm}(\lambda 2)$ are fed into SOA1 and SOA2, respectively, while the set/reset data streams of $1.8 \mathrm{~mW}$ average power each follow the corresponding experimentally employed patterns. The evaluation outputs are depicted in Figure 8(ii), assuming 2-mm intermediate coupling length, and they evidently follow the exact same pattern as the experimentally obtained. For SR-Flip Flop operation with noncomplementary control pulses, they exhibit 13-dB ER and 2.4-dB AM, whereas improved performance of 15-dB ER and 1.3-dB AM is demonstrated in case of complementary set and reset pulses. Although both outputs experience some pattern effect and a slight gain overshoot when switching state, it is clearly shown that full switching can be achieved at $10-\mathrm{Gb} / \mathrm{s}$ operational speed.
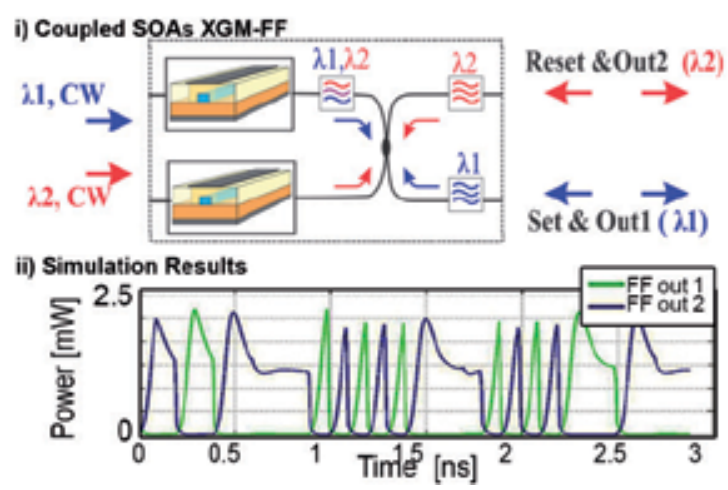

iii) Experimental Results
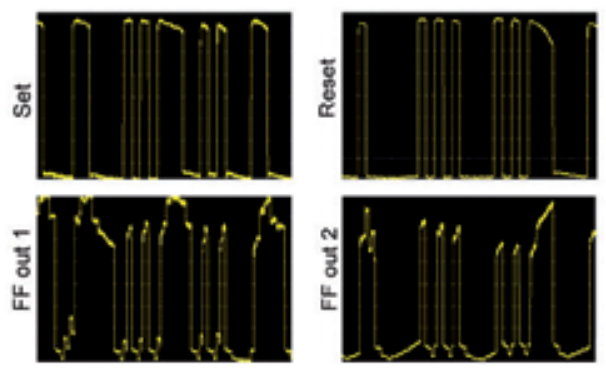

Figure 8. (i) Coupled SOA waveguides operating as XGM switches, in a flip-flop circuit arrangement. (ii) Numerical evaluation of the flip-flop at $10 \mathrm{~Gb} / \mathrm{s}$. (iii) Experimentally obtained traces.

\subsection{SOA-MZI XOR gate exploiting XPM phenomena}

An SOA-MZI gate operating as an all-optical XOR gate exploiting XPM phenomena has been both experimentally and numerically evaluated, as shown in Figure 9. Figure 9(i) illustrates the arrangement of the SOA-MZI XOR gate. The XOR-gate has been experimentally and numerically evaluated with the results illustrated in Figure 9(ii) and (iii), respectively. The 
synchronized numerical time traces demonstrated the proof of principle. Control (Ctr) signal 1 at wavelength $\lambda 2$ and control 2 at $\lambda 3$, respectively, are fed as control signals to the SOA-MZI. The time trace obtained at $\lambda 1$ wavelength at the switching output port of SOA-MZI is illustrated at the third row of numerical traces, where it can be seen that a "logical 1" pulse is obtained, when exclusively one of the input control bits bears a "logical 1 ," while a "logical 0 " pulse is obtained, when the two input control bits are equal. The experimental eye diagram of the XOR output is illustrated in Figure 9(ii), revealing an extinction ratio (ER) of $8 \mathrm{~dB}$ and an amplitude modulation (AM) of $1 \mathrm{~dB}$. Equal performance has been obtained for the simulated eye diagram of the XOR output at Figure 9(ii).

i) SOA-MZI XOR gate

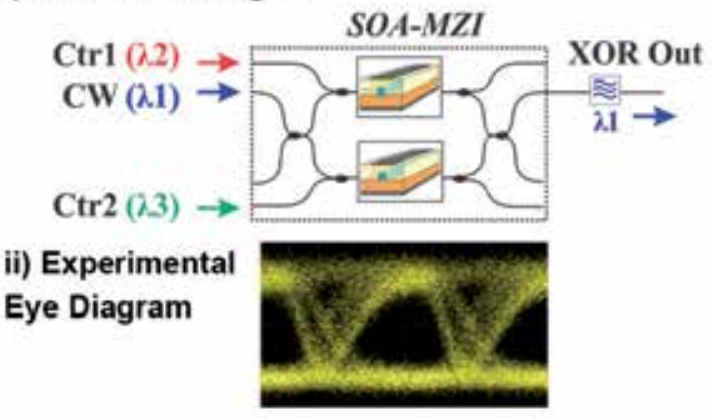

iii) Simulation Results

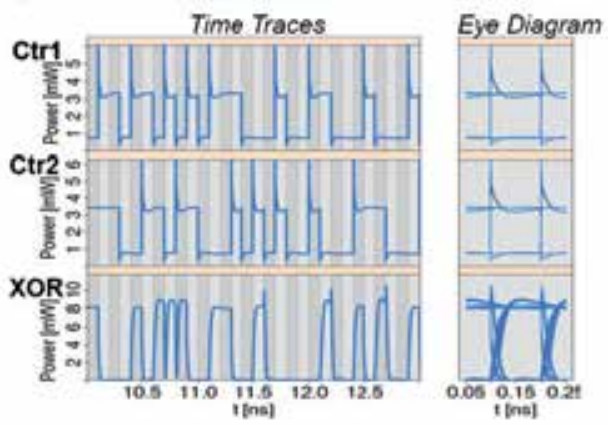

Figure 9. (i) SOA-MZI arrangement operating as an all-optical XPM-based XOR gate, (ii) experimentally obtained eye diagram at the SOA-MZI output, and (iii) synchronized simulation time traces and eye diagrams.

\section{Conclusions}

A holistic methodology approach on time domain numerical modeling has been demonstrated, targeting to address accuracy and efficiency. Accuracy is addressed through the development of an experimentally validated numerical model and a gain parameterization procedure. Following the development of a validated numerical model relying on the TMM analysis technique, qualitative results are presented so as to investigate the gain dynamics and the recovery time of the SOA during pump-probe measurements. Efficiency is sought through the development of a newly introduced time domain SOA modeling technique based on the multigrid concepts to introduce adaptive time stepping.

\section{Acknowledgements}

This work was supported by FP7 MC-IAPP project COMANDER (contract no. 612257). 


\section{Author details}

Christos Vagionas ${ }^{1,2^{*}}$, Jan Bos ${ }^{3}$, George T. Kanellos ${ }^{1,2}$, Nikos Pleros ${ }^{1,2}$ and Amalia Miliou ${ }^{1,2}$

*Address all correspondence to:

1 Department of Informatics, Aristotle University of Thessaloniki, Thessaloniki, Greece

2 Information Technologies Institute, Centre for Research \& Technology Hellas,

Thessaloniki, Greece

3 Phoenix Software, Enschede, Netherlands

\section{References}

[1] Contestabile G Maruta A Sekiguchi S Morito K Sugawara M Kitayama K. All-optical wavelength multicasting in a QD-SOA. IEEE Journal of Quantum Electronics. 2011;47(4):541-547. DOI: 10.1109/JQE.2010.2096500

[2] Buset J M El-Sahn Z A Plant D V. Experimental demonstration of a $10 \mathrm{~Gb} / \mathrm{s}$. IEEE Photonics Technology Letters. 2013;25(15):1435-1438. DOI: 10.1109/LPT.2013.2266615

[3] Scaffardi M Lazzeri E Furukawa H Wada N Miyazak T Poti L Bogoni A. 160 Gb/s/ Port 2x2 OPS node test-bed performing $50 \mathrm{Gchip} / \mathrm{s}$ all-optical active label processing with contention detection. IEEE Journal of Lightwave Technology. 2009;28(6):922930. DOI: 10.1109/JLT.2009.2035524

[4] Willner A E Khaleghi S Chitgarha M R Yilmaz O F. All-optical signal processing. IEEE Journal of Lightwave Technology. 2014;32(4):660-680. DOI: 10.1109/JLT. 2013.2287219

[5] Matsuura M Kishi N Miki T. Ultra wideband wavelength conversion using cascaded SOA-based wavelength converter. IEEE Journal of Lightwave Technology. 2007;25(1):38-45. DOI: 10.1109/JLT.2006.888939

[6] Vagionas C Markou S Dabos G Alexoudi T Tsiokos D Miliou A Pleros N Kanellos GT. Column address selection in optical RAMs with positive and negative logic row access. IEEE Photonics Journal. 2013;5(6):78000410. DOI: 10.1109/JPHOT. 2013.2288299

[7] Lin G R Pan C L Yu K COptimizing the extinction ratio and error bit penalty of the on-off-keying pulse data with gain-constrictive SOA. IEEE Journal of Quantum Electronics. 2009;45(3):264-272. DOI: 10.1109/JQE.2009.2013087 
[8] Teimoori H Topomondzo J D Ware C Erasme D. Optical packet header processing using time-to-wavelength mapping in semiconductor optical amplifiers. IEEE Journal of Lightwave Technology. 2007;25(8):2149-2158. DOI: 10.1109/JLT.2007.899787

[9] Songnian F Shum P Nam Q N Chongqing W Yajie L Chan C C. An enhanced SOAbased double-loop optical buffer for storage of variable-length packet. IEEE Journal of Lightwave Technology. 2008;26(4):425-431. DOI: 10.1109/JLT.2007.912516

[10] Wolfson D Kloch A Fjelde T Janz C Dagens B Renaud M. 40-Gb/s all-optical wavelength conversion, regeneration, and demultiplexing in an SOA-based all-active Mach-Zehnder interferometer. IEEE Photonics Technology Letters. 2000;12(3):332334. DOI: 10.1109/68.826931

[11] Dumon P. ePIXfab, a European platform for silicon photonics R\&D. In: European Conference on Optical Communication; 20-24 Sept. 2009 ; Vienna. IEEE; 2009.

[12] Kish F.A.. Current status of large-scale InP photonic integrated circuits. IEEE Selected Topics in Quantum Electronics. 2011;17(6):1470-1489. DOI: 10.1109/JSTQE. 2011.2114873

[13] Nagarajan R. et al.. Large-scale photonic integrated circuits. IEEE Selected Topics in Quantum Electronics. 2005;11(1):50-65. DOI: 10.1109/JSTQE.2004.841721

[14] Lawniczuk K. et al.. InP-based photonic multiwavelength transmitter with DBR laser array. IEEE Photonics Technology Letters. 2013;25(4):352-354. DOI: 10.1109/LPT. 2013.2238626

[15] Heck M J R et al.. Hybrid silicon photonic integrated circuit technology. IEEE Selected Topics of Quantum Electronics. 2013;19(4):6100117. DOI: 10.1109/JSTQE. 2012.2235413

[16] Sun J Timurdogan E Yaacobi A Su Z Hosseini E S Cole D B Watts M R. Large-scale silicon photonic circuits for optical phased arrays. IEEE Selected Topics of Quantum Electronics. 2014;24(4):8201115. DOI: 10.1109/JSTQE.2013.2293316

[17] Nakamura $S$ et al.. Wavelength selective switching with one-chip silicon photonic circuit including 8× 8 matrix switch. In: OFC/NFOEC; 6-10 March 2011; Los Angeles, CA, USA. IEEE; 2011. p. 1-3.

[18] Poustie A. SOA-based all-optical processing. In: OFC/NFOEC; 25-29 March 2007; Anaheim, CA, USA. IEEE; 2007. DOI: 10.1109/OFC.2007.4348868

[19] Apostolopoulos D et al.. Contention resolution for burst-mode traffic using integrated SOA-MZI gate arrays and self-resetting optical flip-flops. IEEE Photonics Technology Letters. 2008;20(24):2024-2026. DOI: 10.1109/LPT.2008.2005909

[20] Kehayas E et al.. All-optical network subsystems using integrated SOA-based optical gates and flip-flops for label-swapped networks. IEEE Photonics Technology Letters. 2006;18(16):1750-1752. DOI: 10.1109/LPT.2006.880784 
[21] Zhang S et al. Optical shift register based on an optical flip-flop memory with a single active element. In: European Conference Optical Communication; 25-29 September 2005; Glasgow, Scotland. IET; 2005.

[22] Wang J. Meloni G. Berretini G. Poti L. Bogoni A.. All-optical binary counter based on semiconductor optical amplifiers. Optics Letters. 2009;34(22):3517-3519. DOI: 10.1364/OL.34.003517

[23] Francis D A DiJaili S P WalkerJ D. A single-chip linear optical amplifier. In: OFC; 1722 March 2001; Anaheim, CA, USA. IEEE; 2001.

[24] Itoh M Shibata Y Kakitsuka T Kadota Y Tohmori Y. Polarization-insensitive SOA with a strained bulk active layer for network device application. IEEE Photonics Technology Letters. 2002;14(6):765-676. DOI: 10.1109/LPT.2002.1003086

[25] Giannoulis G et al.. Bringing high-performance GaInNAsSb/GaAs SOAs to true data applications. IEEE Photonics Technology Letters. 2015;27(16):1691-1694. DOI: 10.1109/LPT.2015.2436697

[26] Dorgeuiele F Noirie L Faure J P Ambrosy A Rabaron S Boubal F Schilling M Artigue C. $1.28 \mathrm{Tbit} / \mathrm{s}$ throughput $8 \times 8$ optical switch based on arrays of gain-clamped semiconductor optical amplifier gates. In: OFC; 7-10 March 2000 ; Baltimore, MD, USA: IEEE; 2000. DOI: 10.1109/OFC.2000.869463

[27] D. Fitsios, et al.. Dual SOA-MZI wavelength converters based on III-V hybrid integration on a $\mu \mathrm{m}$-scale Si platform. IEEE Photonics Technology Letters. 2014;26(6): 560-563. DOI: 10.1109/LPT.2013.2297404

[28] Kamelian. 1550nm Nonlinear SOA [Internet]. Available from: http://www.kamelian.com/data/nlsoa_ds.pdf [Accessed: 28 September 2015]

[29] Runge P Petermann K Brinker W Schlak M Sartorius B. Supercontinuum generating in ultralong SOAs - theory and experiment. In: European Conference Optical Communication ; 20-24 Sept. 2009 ; Vienna, Austria. IEEE; 2009.

[30] Uskov A Mørk J Mark J. Wave mixing in semiconductor laser amplifiers due to carrier heating and spectral-hole burning. IEEE Journal Quantum Electronics. 1994;30(8): 1769-1781. DOI: 10.1109/3.301641

[31] Connelly M.J. Wideband semiconductor optical amplifier steadystate numerical model. IEEE Journal Quantum Electronics. 2001;37(3):439-447. DOI: 10.1109/3.910455

[32] Vujicic Z Dionisio R P Shahpari A Pavlovic N B Teixeira A. Efficient dynamic modeling of the reflective semiconductor optical amplifier. IEEEE Selected Topics Quantum Electronics. 2013;19(5):3000310. DOI: 10.1109/JSTQE.2013.2259616

[33] Mathlouthi W Lemieux P Salsi M Vannucci A Bononi A Rusch L A. Fast and efficient dynamic WDM semiconductor optical amplifier model. IEEE Journal Lightwave Technology. 2006;24(11):4353-4365. DOI: 10.1109/JLT.2006.884217 
[34] Weng A Yang X Hu W. Theoretical analysis of high-speed all-optical turbo-switches. IEEE Selected Topics Quantum Electronics. 2012;18(2):662-669. DOI: 10.1109/JSTQE. 2011.2134074

[35] Totovic A R Crnjanski J V Krstic M M Masanovic M L Gvozdic D M. A self-consistent numerical method for calculation of steady-state characteristics of traveling-wave and reflective SOAs. IEEE Selected Topics Quantum Electronics. 2010;19(5):14861494. DOI: 10.1109/JSTQE.2013.2263118

[36] Agrawal G P Olsson N A. Self phase modulation and spectral broadening of optical pulses in semiconductor laser amplifier. IEEE Quantum Electronics. 1989;25(11): 2297-2306. DOI: 10.1109/3.42059

[37] Vagionas C Fitsios D Kanellos G T Pleros N Miliou A. Optical RAM and flip-flops using bit-input wavelength diversity and SOA-XGM switches. IEEE Journal of Lightwave Technology. 2012;30(18):3003-3009. DOI: 10.1109/JLT.2012.2210696

[38] Vagionas C Fitsios D Vyrsokinos K. Kanellos G T Miliou A Pleros N.. XPM and XGM-based optical RAM memories: frequency and time domain theoretical analysis. IEEE Journal of Quantum Electronics. 2014;50(8):683-697. DOI: 10.1109/JQE. 2014.2330068

[39] Davis M G O'Dowd R F. A transfer matrix method based large-signal dynamic model for multielectrode DFB lasers. IEEE Journal Quantum Electronics. 1995;30(11): 2458-2466. DOI: $10.1109 / 3.333696$

[40] Matsumoto A Nishimura K Utaka K Usami M. Operational design on high-speed semiconductor optical amplifier with assist light for application to wavelength converters using cross-phase modulation. IEEE Journal of Quantum Electronics. 2006;42(3):313-323. DOI: 10.1109/JQE.2006.869809

[41] Hattori M Nishimura K Inohara R Usami M. Bidirectional data injection operation of hybrid integrated SOA-MZI all-optical wavelength converter. IEEE Journal Lightwave Technology. 2007;25(2):512-519. DOI: 10.1109/JLT.2006.888232

[42] Hairer E Wanner G. Implementation of implicit Runge-Kutta methods. In: Solving Ordinary Differential Equations II: Stiff and Differential-Algebraic Problems. Springer-Verlag Berlin Heidelberg; 1996. DOI: 10.1007/978-3-642-05221-7

[43] Trottenberg U Oosterlee W C Schüller A. Adaptive multigrid. In: Multigrid. London, UK: Academic Press; 2011. p. 356-388.

[44] Vagionas C Bos J. An adaptive stepsize controlled solver for the dynamic WDM semiconductor optical amplifier response. In: SPIE Photonics Europe, Semiconductor Lasers and Laser Dynamics VI; 2 May 2014; Brussels, Belgium. SPIE; 2014. p. 91341T. DOI: $10.1117 / 12.2051778$ 
[45] Gillner L, Goobar E, Thylen L, Gustavsson M. Semiconductor laser amplifier optimization: an analytical and experimental study. IEEE Quantum Electronics. 1989;25(8): 1822-1827. DOI: 10.1109/3.34041

[46] Minch J Park S H Keating T Chuang S L. Theory and experiment of In1-xGaxAsy P1$y$ and In1- $x-y$ GaxAlyAs long-wavelength strained quantum-well lasers. IEEE Quantum Electronics. 1999;35(5):771-782. DOI: 10.1109/3.760325

[47] Xiao S Vogiatzis N Rorison J M. Theoretical study on dilute nitride $1.3 \mu \mathrm{m}$ quantum well semiconductor optical amplifiers: incorporation of $\mathrm{N}$ compositional fluctuations. IEEE Quantum Electronics. 2013;49(10):811-820. DOI: 10.1109/JQE. 2013.2278196

[48] Cheung S Kawakita Y Shang K Yoo S J B. Theory and design optimization of energyefficient hydrophobic wafer-bonded III-V/Si hybrid semiconductor optical amplifiers. IEEE Lightwave Technology. 2013;31(24):4057-4066. DOI: 10.1109/JLT. 2013.2284287

[49] Toptchiyski G Kindt S Peterman K Hillinger E Diez S Weber H G. Time-domain modeling of semiconductor optical amplifiers for OTDM applications. IEEE Lightwave Technology. 1999;17(12):4057-4066. DOI: 10.1109/50.809680

[50] Centre for Integrated Photonics. SOA-NL-OEC-1550-1.55 $\mu \mathrm{m}$ non-linear semiconductor Optical amplifier [Internet]. Available from: http://www.coretk.com/CataLog/ cata_img/FILE/963076829/CIP/166_167_1139895639.pdf [Accessed: 28 September 2015] 

Chapter 2

\title{
Gallium Nitride-based Semiconductor Optical Amplifiers
}

\author{
Rintaro Koda, Hideki Watanabe and Shunsuke Kono \\ Additional information is available at the end of the chapter \\ http://dx.doi.org/10.5772/61664
}

\begin{abstract}
GaN-based material can potentially cover a wide spectral emission range, and laser diodes emitting in the UV, violet, blue, green, and red wavelengths have already been demonstrated and/or commercialized. GaN-based semiconductor optical amplifiers (SOAs) have the ability to boost the output power of laser diodes and thus are candidates for a broad variety of potential uses. Applications that utilize short wavelength, ultrafast pulses, including microprocessing, orthoptics, and next-generation optical storage can most benefit from GaN-based SOAs since current ultrafast pulse sources rely on large, expensive solid-state lasers. GaN-based SOAs can generate high-energy, high peak power optical pulses when used in conjunction with mode-locked laser diodes. In this chapter, the basic characteristics of these devices are discussed, concentrating on pulse amplification. Early experimental work, as well as the latest results, is presented, and improvements in the SOA design allowing the generation of higher optical pulse energy are discussed.
\end{abstract}

Keywords: Gallium nitride, GaN, Semiconductor optical amplifier, SOA, Optical pulse generation

\section{Introduction}

Gallium nitride (GaN)-based optoelectronic devices have attracted significant research interest over the last two decades. Some of these applications, such as light-emitting diodes (LEDs) and ultraviolet (UV), ${ }^{1}$ blue-violet, blue, and green laser diodes (LDs), have already been commercialized and have had an impact on everyday life as solid-state lighting, high-capacity optical storage (such as Blu-ray discs), and display units. The GaN-based material AlGaInN has the potential to cover a particularly wide spectral range, from the deep UV to the infrared (IR). LED emissions at wavelengths as short as $210 \mathrm{~nm}$ have already been reported [1]. In the 
case of LDs, lasing actions over the range of 330-350 nm have been obtained with electrical pumping using $\mathrm{GaN}$ or AlGaN quantum wells [2,3]. In addition, Frost et al. reported lasing at longer wavelengths in the red region of the spectrum (at $630 \mathrm{~nm}$ ) from a InGaN/GaN quantum dot laser [4]. The fact that many researchers have focused on this topic indicates the significant potential of GaN-based optoelectronic devices. However, one important device is missing: the semiconductor optical amplifier (SOA).

An SOA is an important component of optical communication systems, and numerous reports regarding this type of device have been published since its invention in the 1960s. One of the main advantages of the SOA is its ability to be monolithically integrated with other devices. An SOA can be used as a pre-amplifier or a booster amplifier as well as in other functional devices, such as a wavelength converter or optical switch that utilizes its nonlinear nature [5, 6]. An SOA can also be applied as a gain medium to produce short optical pulses in conjunction with a saturable absorber [7]. In high peak power applications, such as two photon bioimaging, an SOA can be used to boost the short optical pulses generated by a mode-locked laser diode (MLLD) $[8,9]$.

High peak power and ultrashort optical pulses have a number of unique applications, many of which utilize the multiphoton processes that are discussed in detail in Section 4 . The majority of pulse sources, however, consist of large, bulky solid-state lasers such as a mode-locked titanium sapphire (Ti:Sap) laser. Moreover, to generate optical pulses at near-UV wavelengths, most of the available pulse sources require wavelength conversion, such that a second- or thirdorder harmonic of an IR pulse is required. This means that more sophisticated laser systems are necessary to obtain UV pulses. Fiber lasers are an alternative to solid-state lasers and have the advantage of a smaller footprint. However, the requirements for wavelength conversion are the same since these devices can only generate photons in the IR wavelength. Such wavelength conversion systems have a number of disadvantages: a larger footprint, the need for precise alignment, less wall-plug efficiency, and increased complexity of the overall system, usually leading to higher costs and longer stabilizing times. For all these reasons, the direct generation of UV pulses is highly desirable, and thus, a GaN-based SOA could be an important device with regard to obtaining short wavelength optical pulses.

As noted, GaN-based devices potentially can generate a very wide range of photons, from the deep UV to the red wavelength of the spectrum. Optical pulse generation in this type of device was first demonstrated in 1997 by Professor Nakamura, the 2014 Noble laureate, using a selfpulsating LD (SPLD) [10]. Since then, many groups have explored GaN-based lasers with the aim of producing optical pulses. Earlier studies concentrated on self-pulsation for applications in optical storage systems since this approach can reduce the optical feedback noise [1--14]. Several pulse generation techniques exist, including gain-switching [1-17], self-pulsating [18-20], mode-locking [7, 17, 21], and superradiance [22]. Kono et al. demonstrated the first gain-switching (GS) operation of a GaN-based LD, producing a peak power of $12 \mathrm{~W}$ and $10 \mathrm{~W}$ with a pulse duration of $10 \mathrm{ps}$ at $405 \mathrm{~nm}[15,17]$. Kuramoto et al. later improved the peak power to $55 \mathrm{~W}$ by optimizing the electron blocking layer in the LD [16]. SPLDs were also explored for applications in high peak power optical sources [18, 20,23], and results included a peak power of $2.4 \mathrm{~W}$ with a bisectional $\mathrm{LD}$ [18], $10 \mathrm{~W}$ and $0.6 \mathrm{~W}$ with a triple section straight 
waveguide LD [19, 20], and $20 \mathrm{~W}$ and $10 \mathrm{~W}$ with a triple section bow tie structure [23, 24]. Mode locking is another means of generating optical pulses and was first demonstrated by Gee and Bowers using a GaN-based SOA as the gain medium [7]. Saito also demonstrated an external cavity passive mode-locked laser diode (MLLD), incorporating a bisectional waveguide structure with a 3-ps pulse duration and a 0.2-W peak power [25]. Oki et al. improved the peak power to $3 \mathrm{~W}$ in the optimized active region [26] and later to $20 \mathrm{~W}$ by employing a flared waveguide structure [21]. The reduction of the pulse duration is important for high peak power generation, and Kono et al. have demonstrated $200 \mathrm{fs}$ pulse generation using a dispersion compensated external cavity and spectral filtering [27]. To further improve the peak power and pulse energy, the use of an SOA is essential. The SOA can be used to boost the output power generated by an LD in a master oscillator power amplifier (MOPA) configuration. Our group has previously developed GaN-based SOAs meant to improve the pulse energy as well as the peak power. In 2010, a peak power over $100 \mathrm{~W}$ with a corresponding pulse energy of approximately $330 \mathrm{pJ}$ was demonstrated, employing an MLLD in conjunction with an SOA [28], and these values were later improved to approximately $300 \mathrm{~W}$ and $590 \mathrm{pJ}$ [29]. Recently, an SOA incorporating a widely flared waveguide structure was developed, and the peak power and the pulse energy were further improved to $2.2 \mathrm{~nJ}$ and $630 \mathrm{~W}$ [30]. The pulse characteristics of published GaN-based devices are summarized in Figure 1 and Table 1 (note that earlier studies of SPLDs were excluded from these summaries since pulse durations were not available). It can be clearly seen that the use of an SOA significantly improves the peak power as well as the pulse energy.

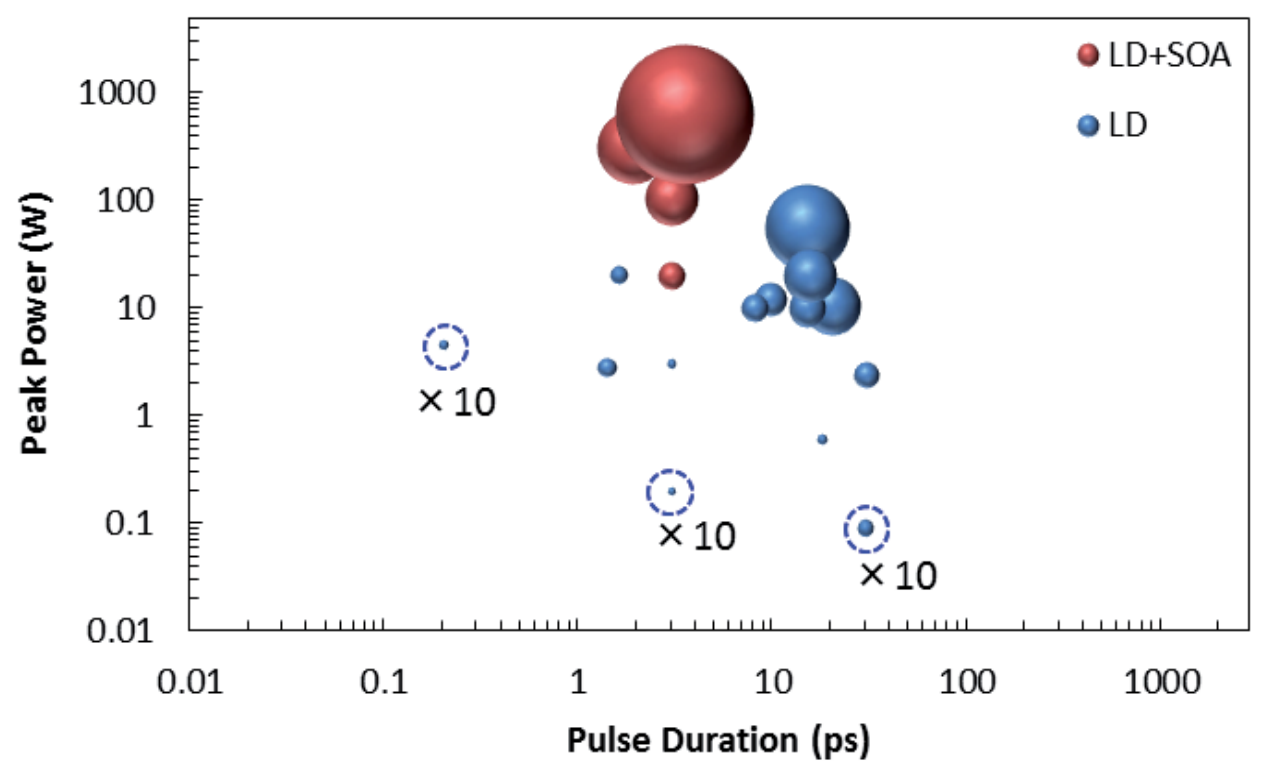

Figure 1. Peak power vs. pulse duration for GaN-based devices. The size of each circle indicates the optical pulse energy. The use of an SOA effectively increases both the peak power and pulse energy. Note that in the case of those data points indicated by dashed circles, the pulse energies are multiplied by 10 for illustrative purposes. 


\begin{tabular}{|c|c|c|c|c|c|c|c|}
\hline Device type & $\begin{array}{c}\text { Pulse } \\
\text { Energy } \\
\text { (pJ) }\end{array}$ & $\begin{array}{c}\text { Peak Power } \\
\text { (W) }\end{array}$ & $\begin{array}{c}\text { Average } \\
\text { power }(\mathrm{mW})\end{array}$ & $\begin{array}{c}\text { Pulse } \\
\text { Repetition } \\
\text { (GHz) }\end{array}$ & $\begin{array}{c}\text { Pulse } \\
\text { Duration } \\
\text { (ps) }\end{array}$ & $\begin{array}{c}\text { Wavelength } \\
(\mathrm{nm})\end{array}$ & Reference \\
\hline \multirow{2}{*}{ Gain-switching LD } & 116 & 12 & 0.01 & 0.0001 & 9.7 & 405 & [15] \\
\hline & 820 & 55 & .82 & .001 & 15 & 401 & [16] \\
\hline \multirow{5}{*}{ Self-pulsating LD } & 72 & 2.4 & 65 & 0.9 & 30 & 407 & {$[18]$} \\
\hline & $11^{1}$ & 0.6 & - & 4.5 & 18 & 414 & [19] \\
\hline & 150 & 10 & 150 & 1 & 15 & 402 & [20] \\
\hline & $80^{2}$ & 10 & $80^{1}$ & 1.7 & 8 & - & [24] \\
\hline & 310 & 20 & 260 & 0.84 & 15.5 & 406 & [23] \\
\hline \multirow{5}{*}{ Mode-locked LD } & 2.8 & 0.09 & 2 & 0.72 & 30 & 408.5 & [7] \\
\hline & 0.6 & 0.2 & 0.6 & 1 & 3 & 404.5 & [25] \\
\hline & 8.9 & 3 & 8.9 & 3 & 1 & 404 & [26] \\
\hline & 32 & 20 & 32 & 1 & 1.6 & 403.5 & {$[21]$} \\
\hline & 0.9 & 4.5 & 0.9 & 1 & 0.2 & 401 & [27] \\
\hline \multirow{4}{*}{$\begin{array}{l}\text { Mode-locked LD } \\
\text { + SOA }\end{array}$} & 78 & 20 & 78 & 1 & 3 & 404 & {$[31]$} \\
\hline & 329 & 103 & 329 & 1 & 3 & 404.3 & [28] \\
\hline & 586 & 308 & 586 & 1 & 1.9 & 405.4 & [29] \\
\hline & 2200 & 630 & 710 & 0.812 & 3.5 & 405.2 & [30] \\
\hline
\end{tabular}

${ }^{1}$ : Estimated using the peak power, pulse repetition and pulse duration values provided in the paper.

2: Estimated by using values provided in the paper

Table 1. Summary of the GaN-based optical pulse sources developed by various research groups.

\section{Characterization of continuous wave light amplification}

In this section, the basic characteristics of GaN-based SOAs are reviewed.

\subsection{Basic structures}

In general, there are two types of SOA: the Fabry-Perot type and the travelling-wave type. A Fabry-Perot SOA has nonzero facet reflectivity so that signal light is amplified as the result of many passes through the amplifier. In contrast, a travelling-wave SOA has negligible facet reflectivity such that the signal passes through only once (single-pass). A travelling-wave SOA is simpler to use, and so these devices are discussed exclusively in the present chapter. GaNbased SOAs that we would discuss throughout this chapter consist of a double quantum well structure with $\mathrm{Ga}_{0.92} \mathrm{In}_{0.08} \mathrm{~N}$ quantum wells and $\mathrm{Ga}_{0.98} \mathrm{In}_{0.02} \mathrm{~N}$ barriers grown on an $\mathrm{n}-\mathrm{GaN}$ 
substrate by metal organic chemical vapor deposition (MOCVD). The layer structure is shown in Figure 2. In a travelling-wave SOA, it is important to minimize facet reflectivity, and this is illustrated in Figure 3. Both devices shown in this figure are ridge waveguide structures with a length of $1 \mathrm{~mm}$ and both incorporate facets with anti-reflection (AR) coatings. Waveguides are straight (Figure 3(a)) and angled by $5^{\circ}$ (Figure 3(b)). As the result of nonzero reflectivity, the straight waveguide exhibits significant resonance and a multilongitudinal mode, both of which are undesirable with regard to SOA operation. The incorporation of the angled waveguide effectively reduces back reflection from the facets and thus suppresses the resonance effect.

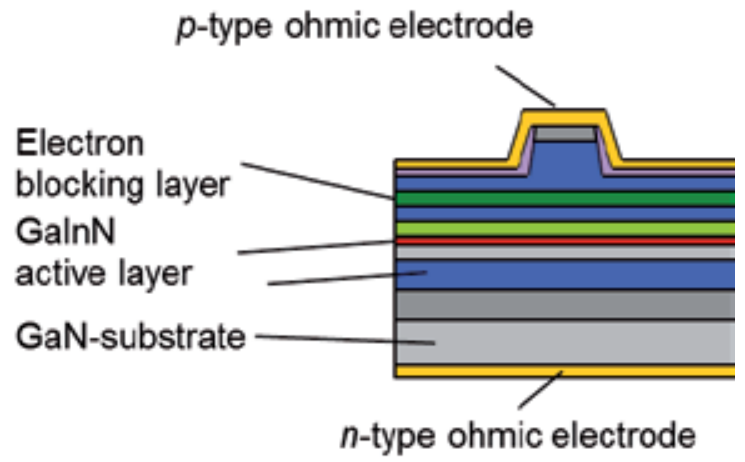

Figure 2. Schematics of the layer structure of the SOAs discussed in the present chapter.
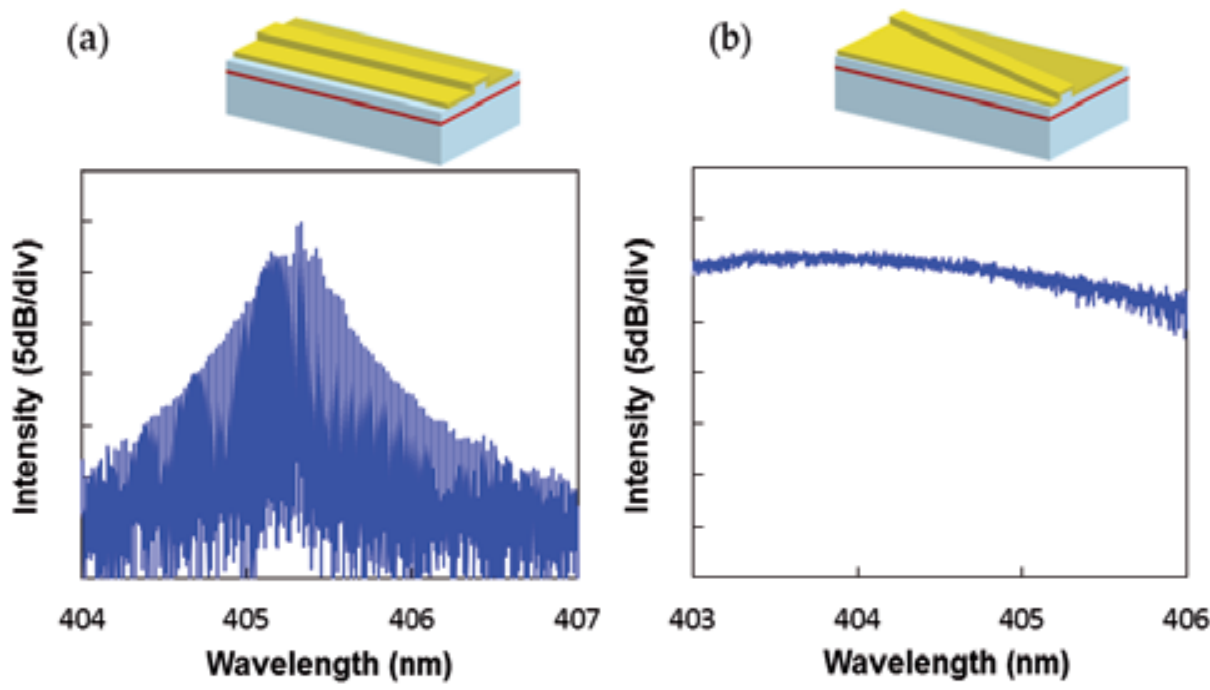

Figure 3. Optical spectra obtained from $1 \mathrm{~mm}$ long SOAs with either (a) straight or (b) angled waveguides. Although both structures had AR-coated faces, the straight waveguide exhibits strong resonance due to residual reflections from an imperfect AR coating. 
The output power of an SOA is limited by its saturation power, $P_{\text {sat }}$ which is given by

$$
P_{\text {sat }}=\frac{h v d w}{\Gamma a \tau}
$$

where $h$ is the Plank constant, $v$ is the signal optical frequency, $d$ and $w$ are the active region thickness and width, respectively, $\Gamma$ is the confinement factor, $a$ is the differential gain, and $\tau$ is the carrier lifetime. Equation (1) indicates that a larger active region cross section, a lower confinement factor, a lower differential gain, and a reduced carrier lifetime are desirable for high power applications. However, the output power is independent of device length. Equation (1) can be interpreted qualitatively, that is, the amount of energy that can be stored in the device is important for higher output from the SOA.

The basic amplification characteristics of an SOA were assessed using device A shown in Figure 4, incorporating a flared waveguide. The input and the output waveguide widths were 1.4 and $5 \mu \mathrm{m}$, respectively, and both facets were AR coated. As noted earlier, the waveguide was angled at $5^{\circ}$ to suppress the resonance effect.

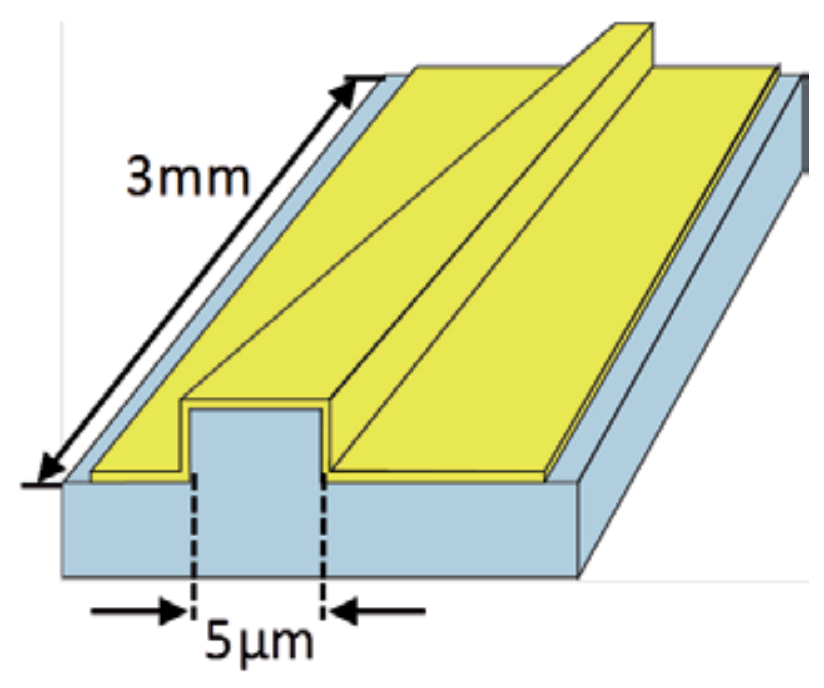

Figure 4. Schematics of device A.

\subsection{Continuous wave amplification characteristics}

In the amplification of continuous-wave (CW) light by the SOA, device A was assessed using the experimental setup shown in Figure 5. The resulting net gain (sometimes also known as the fiber-to-fiber gain) and output power at an input wavelength of $404 \mathrm{~nm}$ are presented as functions of input power at operating currents of 3,4 , and $5 \mathrm{kA} / \mathrm{cm}^{2}$ in Figure 6 . The net gain values were estimated using an optical band-pass filter (BPF) to reject the out-of-band 
components of the amplified spontaneous emission (ASE). It should be noted that the net gain values in this figure include the input and output coupling losses. It is evident that a gain of over $20 \mathrm{~dB}$ was obtained from a small signal input of less than $1 \mathrm{~mW}$, a result that is comparable to the performance of SOAs fabricated with other materials $[5,6]$.

Wavelength dependencies were characterized by tuning the input wavelength to 403,405 , or $407 \mathrm{~nm}$, and the corresponding spectra are presented in Figure 7, in which very different spectra are observed at different input wavelengths. At the shorter wavelength, the amplified spectrum shows that ASE is present, whereas less ASE is observed from amplification at longer wavelengths of 405 and $407 \mathrm{~nm}$. Figure 8(a) plots the output power and ASE spectra, while Figure $8($ b) presents the net gain spectra obtained at different input powers. It is interesting to note that the gain peak shifts to longer wavelength as the input power is increased. At lower input power, the wavelength of the gain peak and the maximum output power are nearly equal to the ASE peak, while higher input power results in a red shift of these values. This phenomenon can possibly be explained by considering that gain saturation due to carrier depletion leads to intra-band relaxation of the carriers. This results in more carriers available for longer wavelength amplification. In addition, GaN-based systems are known to have higher effective masses of electrons and holes [32,33], and this may also play a role in this mechanism.

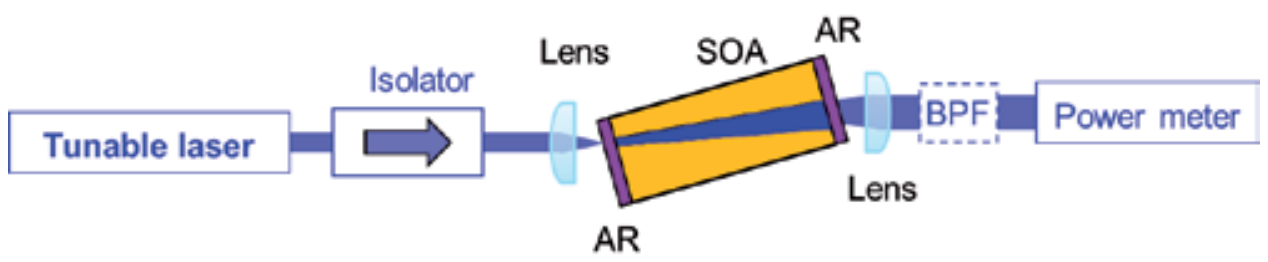

Figure 5. Experimental setup for CW amplification.
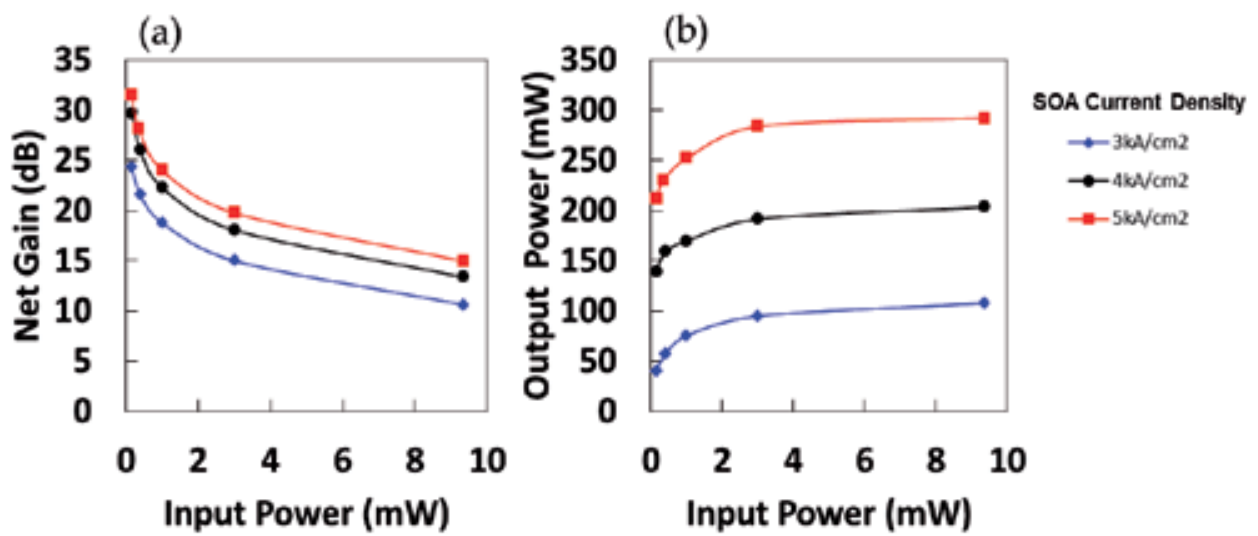

Figure 6. Net gain (left) and output power (right) obtained from the SOA (device A) as functions of CW input power for current densities of 3,4 , and $5 \mathrm{kA} / \mathrm{cm}^{2}$ at an input wavelength of $404 \mathrm{~nm}$. 
(a)

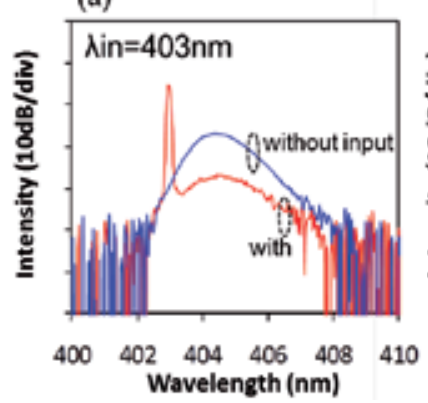

(b)

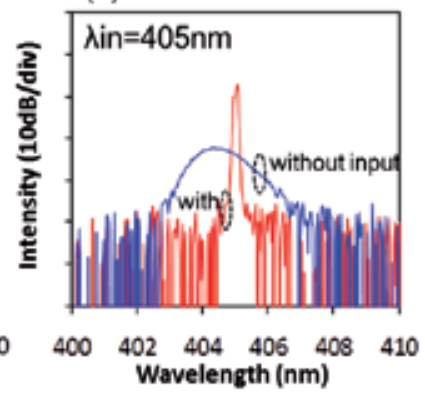

(c)

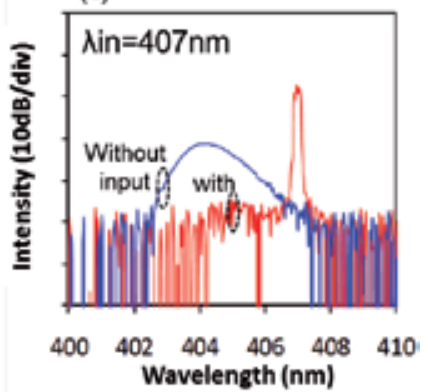

Figure 7. Optical spectra of the output of an angled SOA obtained with input wavelengths of (a) 403, (b) 405, and (c) $407 \mathrm{~nm}$. Current density and input power were $4 \mathrm{kA} / \mathrm{cm}^{2}$ and $11 \mathrm{~mW}$, respectively. Spectra with (red) and without (blue) input are shown for each wavelength.
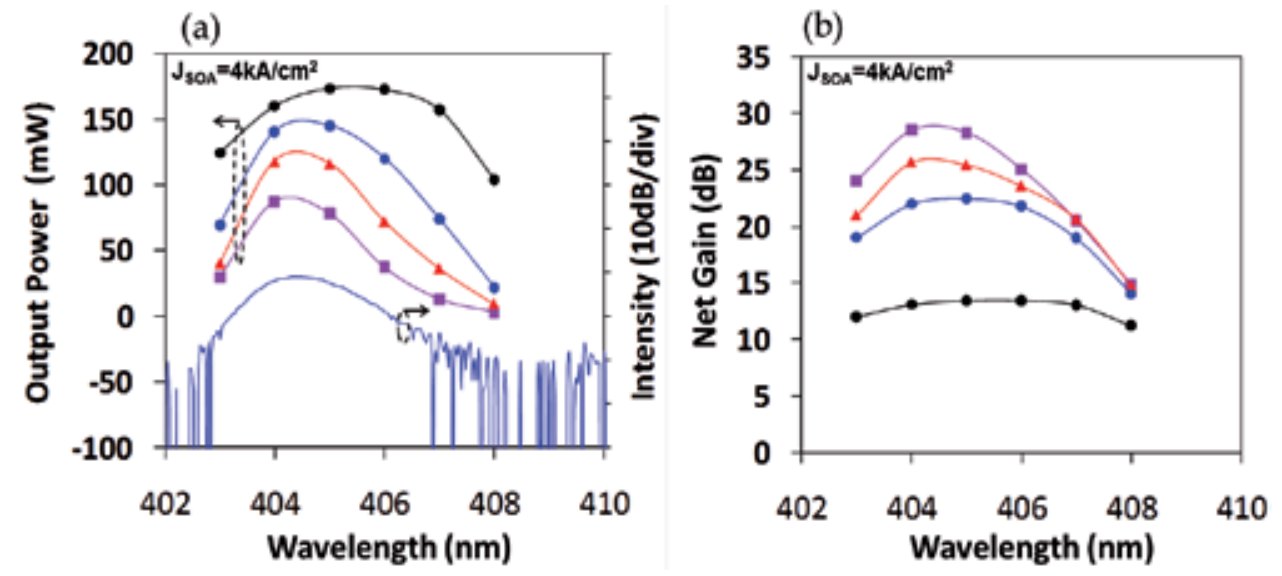

Figure 8. (a) Output power and ASE spectra and (b) net gain spectra obtained from device A at input powers of $11 \mathrm{~mW}$ (black), $1.2 \mathrm{~mW}$ (blue), $0.4 \mathrm{~mW}$ (red), and $0.2 \mathrm{~mW}$ (violet).

\section{Pulse amplification}

\subsection{Limiting factor}

Pulse amplification was initially studied using device A [31], employing a GaN-based external cavity MLLD developed by our group [26]. In this work, a GaN-based LD having the same GaInN active region as the SOA was grown by MOCVD. The resulting LD chip was composed of a forward bias gain section and a reverse bias saturable absorption (SA) section. The facets had a high reflection coating on the SA side and an AR coating on the other side. More details regarding this MLLD are available in the literature [26]. Figure 9 shows the output power of the device as a function of the average input power for both $\mathrm{CW}$ and pulse inputs at a 3- 
$\mathrm{kA} / \mathrm{cm}^{2} \mathrm{SOA}$ bias current density. From this figure, it is evident that significantly lower average power was obtained when applying pulse amplification. To investigate the limiting factors for pulse amplification, we acquired optical spectra as well as temporal characteristics using an optical spectrum analyzer (OSA) and a streak camera. Figure 10(a) presents the optical spectra and Figure 10(b) shows streak camera images obtained from amplified pulses at 2 and 4 $\mathrm{kA} / \mathrm{cm}^{2} \mathrm{SOA}$ bias current densities. The optical spectra of the amplified pulses exhibit a peak wavelength shift toward longer wavelengths as well as an oscillatory structure, representing self-phase modulation (SPM) [34]. High peak power optical pulses induce carrier depletion, which in turn varies the refractive index. At the lower current density of $2 \mathrm{kA} / \mathrm{cm}^{2}$, the streak camera image shows clean optical pulses at a repeat rate of $1 \mathrm{GHz}$. In contrast, at the higher current density $\left(4 \mathrm{kA} / \mathrm{cm}^{2}\right)$, ASE appears between pulses. The optical spectra also changes at the higher current density, such that a broad fifth peak appears near $403 \mathrm{~nm}$. Careful examination of this short wavelength peak using the streak camera indicated that it is a combination of ASE and pulse elements. The carrier recovery time was determined to be less than $300 \mathrm{ps,}$ and thus significantly faster than the pulse repetition of $1 \mathrm{~ns}$. The duration of the amplified pulse was 3 ps and the peak power was estimated to be only $20 \mathrm{~W}$.

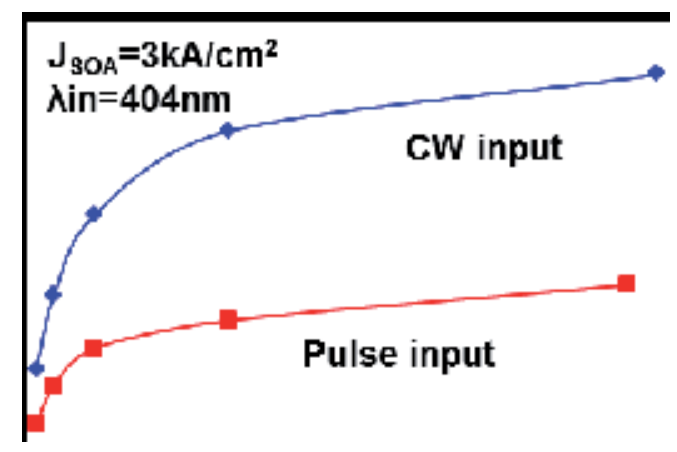

Figure 9. Average output power of the SOA (device A) as a function of input power for CW (diamonds) and pulse (squares) inputs at an SOA current density of $3 \mathrm{kA} / \mathrm{cm}^{2}$ and an input wavelength of $404 \mathrm{~nm}$. Significant reduction of gain is observed for pulse amplification.

\subsection{Improving peak power}

Investigation of device A indicated that intense ASE appeared as the operating current density was increased. Intense ASE leads to gain depletion, preventing efficient amplification of optical pulses. In this process, carrier depletion occurring via a stimulated emission process is induced by the intense ASE, which in turn results in fewer carriers available for pulse amplification. This generates the limited peak power of only $20 \mathrm{~W}$. The fact that intense ASE is a limiting factor for an SOA has also been noted in previous studies of other systems, both theoretically and experimentally [35-37]. To overcome this limitation, intense ASE has to be reduced. However, a trade-off exists since ASE is also needed for optical gain. We have thus pursued the development of SOAs by optimizing device structures so as to obtain low ASE while maintaining sufficient gain. Peak powers were improved to approximately $100 \mathrm{~W}$ and later to 
(a)

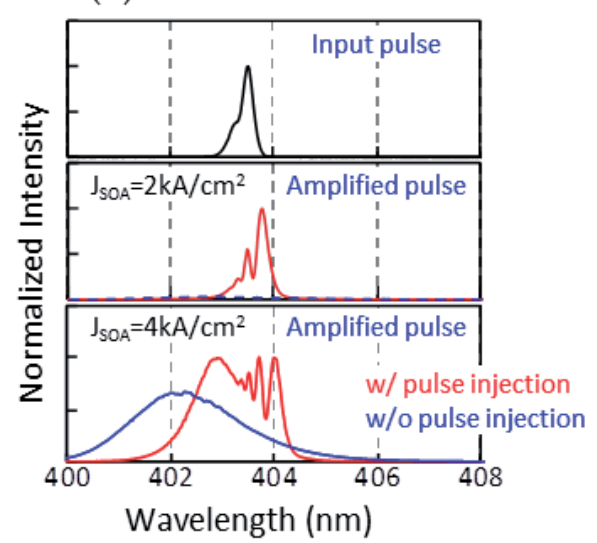

(b)

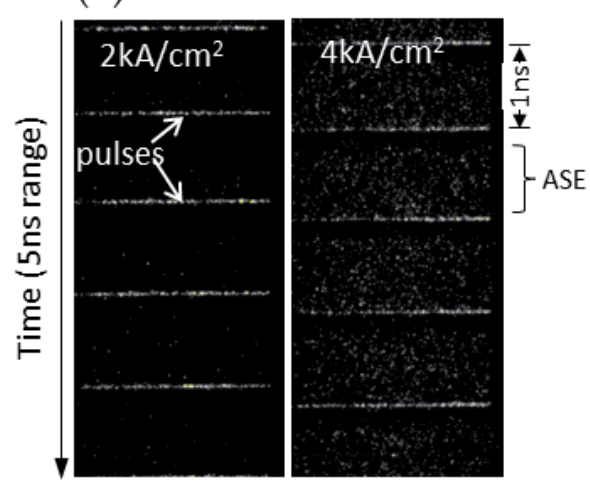

Figure 10. (a) Optical spectra obtained with input and amplified output and (b) streak camera images at SOA current densities of 2 and $4 \mathrm{kA} / \mathrm{cm}^{2}$. At the higher current density, intense ASE is observed between pulses.

$300 \mathrm{~W}$ by optimizing device structure such as the length and optical confinement factors [28, 29]. The corresponding optical pulse energies were approximately 330 and 590 pJ. Recently, further improvements gave a pulse energy of $2200 \mathrm{pJ}$ (corresponding to a peak power of 630 W) [30]. This is the highest ever pulse energy reported for a GaN-based all-semiconductor pulse source. Details of this latest device are presented in the following sections. The SOAs described above are illustrated in Figure 11 as devices B, C, and D. It is known that optical pulse amplification is governed by the saturation energy, $E_{\text {sat }}$ of the SOA [34], and that it can be expressed as follows.

$$
E_{\mathrm{sat}}=\frac{h v d w}{\Gamma a}
$$

The parameters $d, w$, and $\Gamma$ can be readily tuned by adjusting the epitaxial layer structure and waveguide design. In the case of devices $B, C$, and $D$, the values of $d w / \Gamma$ are $8 \times 10^{3}, 9 \times 10^{3}$, and $6 \times 10^{4} \mu \mathrm{m}^{2}$, respectively. Each of these devices was grown by MOCVD, and all have a ridge waveguide structure. Both B and C employ waveguides that are linearly tapered from $1.4 \mu \mathrm{m}$ at the input facet to $15 \mu \mathrm{m}$ at the output facet. In the case of $\mathrm{D}$, the waveguide consists of two sections: the straight preamplifier and the flared main amplifier sections. The lengths are 2, 2.5 , and $3 \mathrm{~mm}$ for $\mathrm{B}, \mathrm{C}$, and $\mathrm{D}$, respectively, and more detailed descriptions have been previously published [2--30]. Figure 12 summarizes the net gain and ASE power values as functions of the SOA current density. For each sample, the net gain is seen to increase with current density but to saturate as the ASE becomes too high. It should be noted that this discussion is not intended to offer a detailed comparison between the devices since the operating conditions under which these data were collected, such as temperature, input pulse width, and power, were not all the same. Rather, the intention is to show the effectiveness of 
varying the parameter $d w / \Gamma$, which governs the optical pulse energy. Figure 13 plots the optical pulse energy as a function of this term.
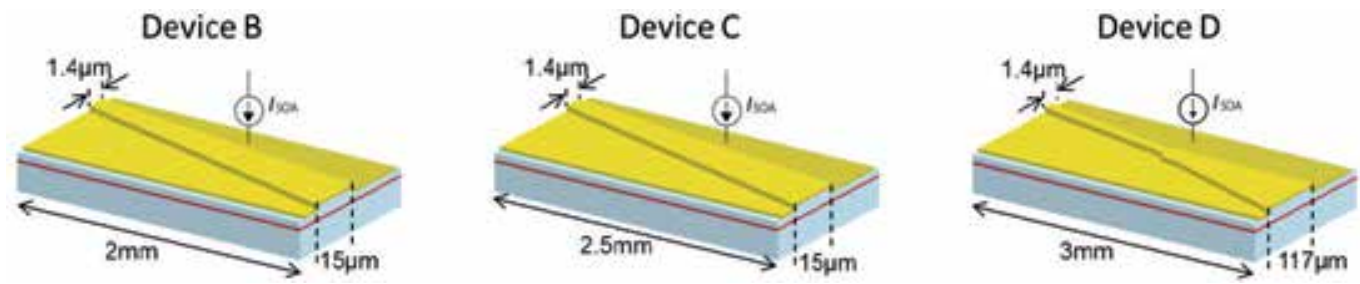

Figure 11. Schematics of three additional SOA designs. The peak power and pulse energy values were approximately 100,300 , and $630 \mathrm{~W}$ and 330, 590, and $2200 \mathrm{pJ}$ for devices B, C, and D, respectively.
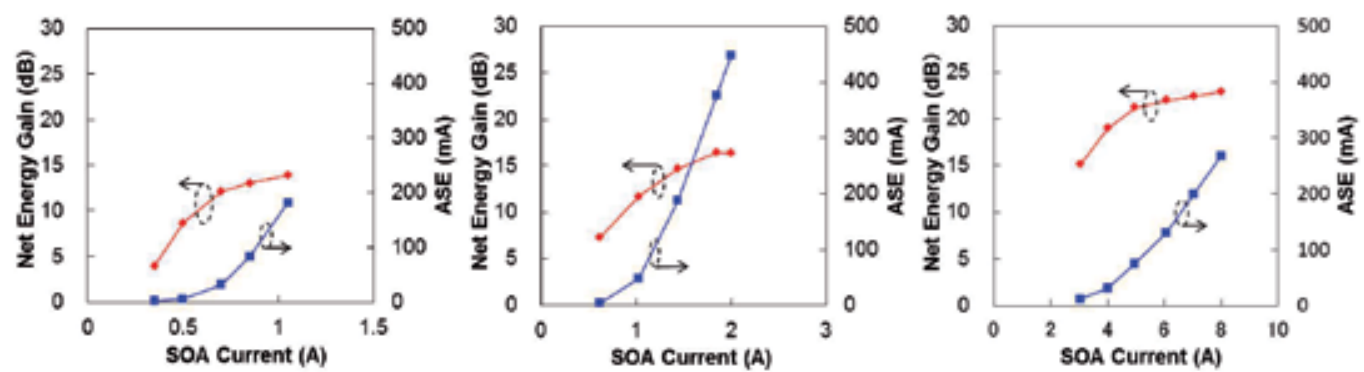

Figure 12. Net gain and ASE power values as functions of SOA current for devices B, C, and D.

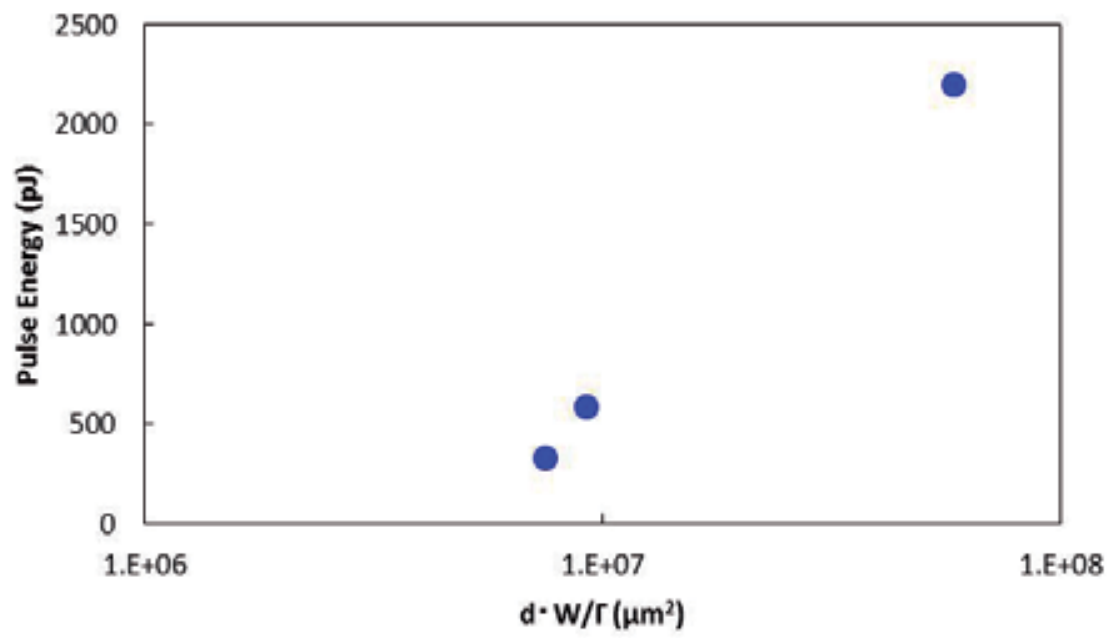

Figure 13. Pulse energy as a function of $d w / \Gamma$. 
It is helpful to discuss in detail the result obtained with device D. This device employed a passively mode-locked GaN laser as the master oscillator, in the commonly used Littrow configuration. The gain section of the LD was forward biased by $90 \mathrm{~mA}$, and the SA section was reverse biased at $-9 \mathrm{~V}$. The pulse repetition rate was $812 \mathrm{MHz}$, and the input power was $7.2 \mathrm{~mW}$. The wavelength was tuned to $405 \mathrm{~nm}$. Temporal characterization of the input pulse was performed using an in-house intensity autocorrelation measurement apparatus that utilizes the surface second harmonics of a $\beta-\mathrm{BaB}_{2} \mathrm{O}_{4}(\mathrm{BBO})$ crystal [38]. Autocorrelation measurements indicated an FWHM value of 4.2 ps, corresponding to a 3.0-ps pulse duration assuming a Gaussian waveform. The peak power was estimated to be $3 \mathrm{~W}$.

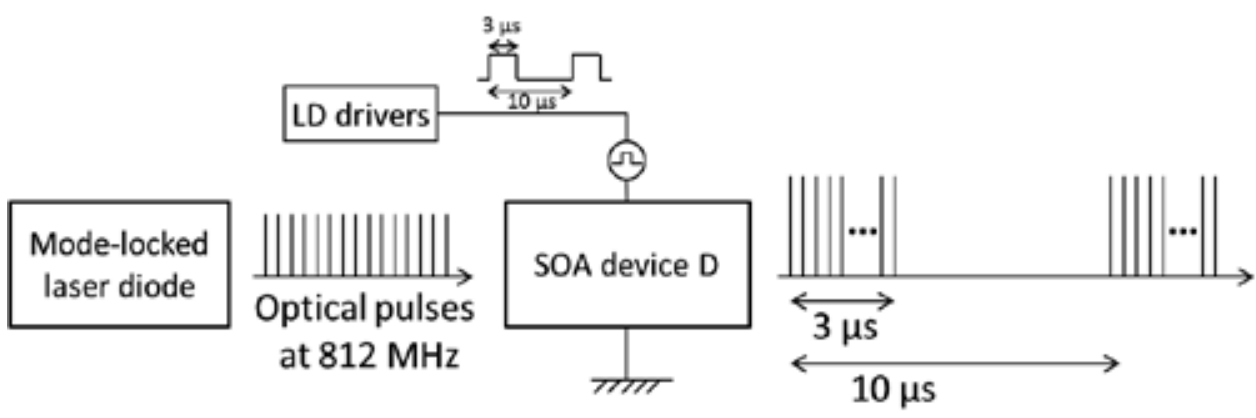

Figure 14. Schematics of the master oscillator power amplifier using SOA device D.

SOA D was driven by LD drivers at a $100-\mathrm{kHz}$ repetition rate with $3 \mu$ s width rectangular current pulse, as illustrated in Figure 14, which presents the autocorrelation trace and optical spectrum acquired at a $8-\mathrm{kA} / \mathrm{cm}^{2}$ bias current density. Here, the FWHM was $5.0 \mathrm{ps}$, and the pulse duration was estimated to be 3.5 ps, assuming a Gaussian waveform. Even though a high net gain of greater than $20 \mathrm{~dB}$ was obtained, the pulse duration was not significantly broadened. The peak wavelength of the amplified pulse was $405.2 \mathrm{~nm}$, indicating a $0.2-\mathrm{nm}$ red shift, as shown in Figure 15(b). The spectral bandwidth also increased from 0.08 to $0.26 \mathrm{~nm}$ after amplification. These spectral changes were due to SPM. It is important to point out that the amount of red shift exhibited in this case was less than that observed in previous trials; as an example, a shift of $0.4 \mathrm{~nm}$ was obtained with device $C$ [29]. Figure 16 shows the average output power and estimated pulse energy values as functions of the SOA bias current density. The average output power values were 0.71 and $0.27 \mathrm{~W}$ with and without pulse injection. At $8 \mathrm{kA} / \mathrm{cm}^{2}$, the pulse energy was estimated to be $2.2 \mathrm{~nJ}$, and the corresponding peak power was estimated to be $630 \mathrm{~W}$. These are the highest pulse energy and peak power ever generated by GaN-based MOPA.

\section{Potential applications}

There are many potential applications for GaN-based SOAs, among which those utilizing optical pulses are the most prominent. In this section, these applications are discussed. 
(a)

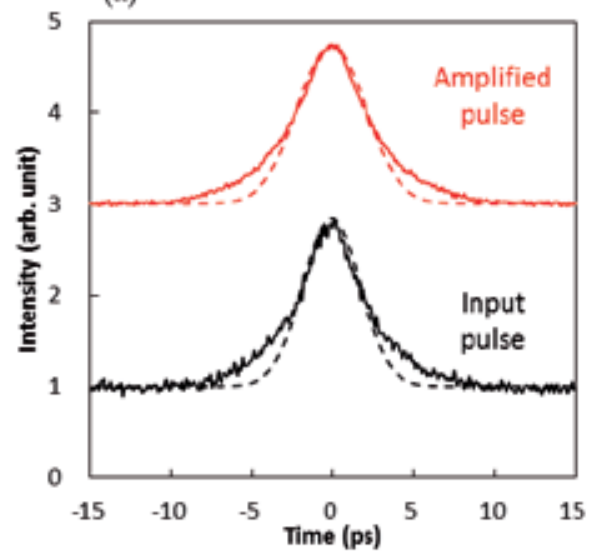

(b)

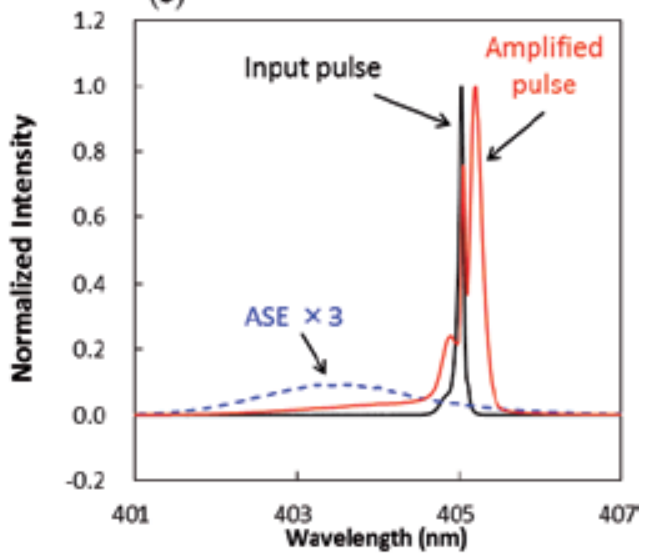

Figure 15. (a) Autocorrelation traces and (b) optical spectra of input and amplified pulses. The dashed lines show Gaussian function fitted to the autocorrelation traces in panel a. The ASE spectrum (with its intensity multiplied by three for illustrative purposes) is also shown in panel $\mathrm{b}$ as a dashed line.
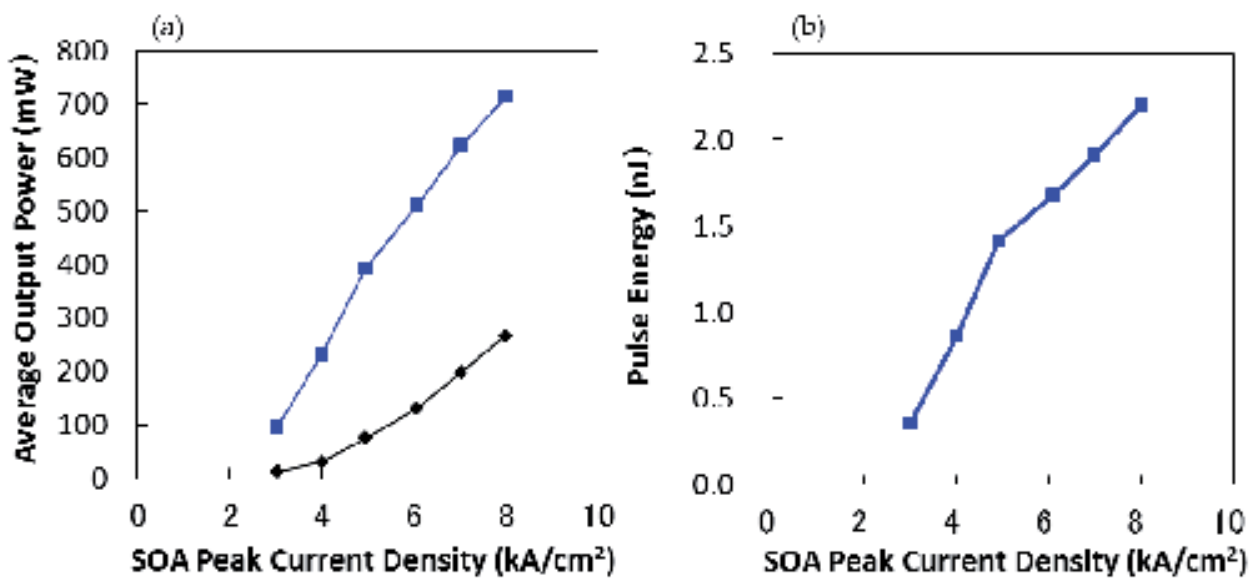

Figure 16. (a) Average output and (b) pulse energy values as functions of the SOA bias current density.

\subsection{Micromachining applications}

Recently, there has been a rapid growth in laser material processing, as the cost of lasers has decreased and fiber laser technology has advanced. In microscale machining, short optical pulses are desirable since picosecond/femtosecond pulses can effectively reduce the heat damage resulting from the laser light. In fact, it has been demonstrated that nanosecond pulses produce dull edges [39], as shown by Chichkov et al., who demonstrated this by comparing the pulse width dependence of laser ablation of steel.

Short wavelength optical pulses can also be beneficial with regard to precise processing since the spot size of the focused beam is proportional to NA/ $\lambda$, where NA is the numerical aperture 
of the lens and $\lambda$ is the wavelength of the laser. Kauf et al. published a systematic study of microprocessing using mode-locked picosecond and q-switched nanosecond UV lasers having the same average and peak power values, although with differing pulse energies [40]. These trials demonstrated that picosecond UV pulses offer an advantage compared to nanosecond pulses since they allow higher cutting speeds when working with dielectric materials such as polyimides. The significantly higher pulse repetition of the mode-locked laser operating at 80 $\mathrm{MHz}$ resulted in a higher cutting speed compared to that obtained from the q-switched laser working at $80 \mathrm{~Hz}$.

\subsection{Orthoptics}

Laser refractive surgery, such as laser in situ keratomileusis (LASIK), corrects the refractive properties of the cornea. Two different types of pulse lasers are typically used in LASIK: an IR femtosecond laser for cutting corneal flaps and a UV nanosecond excimer laser for ablating corneal tissue. Ablation of the corneal tissue with UV pulses (typically at $193 \mathrm{~nm}$ ) is able to change the shape of the cornea's surface to allow for vision correction. Recently, an alternative method for vision correction was demonstrated by $\mathrm{Xu}$ and Knox et al. Instead of physically removing a part of cornea, this new noninvasive technique modifies the refractive index of the cornea using femtosecond pulses at $400 \mathrm{~nm}$, which is the most effective wavelength. The 400$\mathrm{nm}$ pulses are provided by the second-order harmonics of a mode-locked Ti:Sap laser with an average power of $60 \pm 1 \mathrm{~mW}$ and corresponding pulse energies of approximately $0.8 \mathrm{~nJ}$ at the cornea. A GaN-based SOA employing MLLD would seem to be a promising candidate for this application since the required average power and pulse energies can easily be generated by the type of SOA discussed in the present chapter and the associated semiconductor-based system offers energy efficiency and compactness.

\subsection{Optical storage}

The storage capacities of optical disks have improved from 0.7 gigabytes (GB) in the case of compact discs (CDs) to 4.7 GB for digital versatile discs (DVDs) and, more recently, to 25 GB with Blu-ray discs. These improvements were realized in conjunction with advances in laser diode technologies in terms of achieving shorter lasing wavelengths since the spot size of the laser beam is proportional to $\lambda / \mathrm{NA}$. For next-generation optical data storage, three-dimensional (3D) optical data storage might be the best candidate since, as the name implies, this technique allows information to be stored three dimensionally, generating a significant increase in storage capacity [41]. Several groups have demonstrated such systems $[42,43]$, and 1 terabyte data storage has been demonstrated [44]. To store data three dimensionally, multiphoton processes, such as two photon absorption, must be induced using high peak power optical pulses. A GaN-based SOA incorporating MLLD would be highly suitable as a practical pulse source, and in fact, such applications have already been demonstrated $[45,46]$. 


\section{Conclusion}

GaN-based optoelectronic devices are already widely used as optical storage lasers and solidstate lighting, and because of their ability to potentially cover a wide spectral range from the deep UV to the IR regions, many research studies concerning these devices are ongoing. However, very few reports on GaN-based SOAs exist. In this chapter, the basic characteristics of GaN-based SOAs were reviewed, focusing on pulse amplification. The particular SOA devices described herein generate a pulse energy greater than $2 \mathrm{~nJ}$, a value that is comparable to the values obtained from widely used pulse sources based on solid-state lasers. There are many potential applications for these devices, such as microprocessing, orthoptics, and highdensity optical storage, using UV picosecond and femtosecond pulses. These applications would all benefit from employing GaN-based SOAs.

\section{Acknowledgements}

The authors wish to gratefully acknowledge Professor Yokoyama of Tohoku University for helpful discussions and to note that earlier studies of the GaN-SOAs (devices A, B, and C) were performed in collaboration with Professor Yokoyama's research group. Thanks are also due to the author's research colleagues: T. Oki, Y. Takiguchi, M. Kuramoto, T. Miyajima, H. Nakajima, M. Shiozaki, Y. Hanzawa, N. Sugawara, and M. Ikeda. Finally, the assistance of Sony Semiconductor in fabricating the experimental devices is gratefully acknowledged.

\section{Author details}

Rintaro Koda*, Hideki Watanabe and Shunsuke Kono

*Address all correspondence to: Rintaro.Koda@jp.sony.com

Semiconductor Device Development Division, Sony Corporation, Japan

\section{References}

[1] Taniyasu Y, Kasu M, Makimoto T: An aluminium nitride light-emitting diode with a wavelength of 210 nanometres. Nature. 2006;441(7091):32--8.

[2] Yamashita Y, Kuwabara M, Torii K, Yoshida H: A 340-nm-band ultraviolet laser diode composed of GaN well layers. Optics Express. 2013;21:3133. 
[3] Yoshida H, Yamashita Y, Kuwabara M, Kan H: Demonstration of an ultraviolet 336 nm AlGaN multiple-quantum-well laser diode. Applied Physics Letters. 2008;93(24): 241106.

[4] Frost T, Banerjee A, Sun K, Chuang SL, Bhattacharya P: InGaN/GaN quantum dot $\operatorname{red}(\lambda=630 \mathrm{~nm})$ laser. IEEE Journal of Quantum Electronics. 2013;49:923.

[5] Connelly MJ. Semiconductor Optical Amplifiers: Springer US; 2007.

[6] Dutta NK, Wang Q. Semiconductor Optical Amplifiers: World Scientific Pub.; 2006.

[7] Gee S, Bowers JE: Ultraviolet picosecond optical pulse generation from a modelocked InGaN laser diode. Applied Physics Letters. 2001;79:1951.

[8] Ding Y, Aviles-Espinosa R, Cataluna MA, Nikitichev D, Ruiz M, Tran M, et al.: High peak-power picosecond pulse generation at $1.26 \mu \mathrm{m}$ using a quantum-dot-based external-cavity mode-locked laser and tapered optical amplifier. Optics Express. 2012;20(13):14308-20.

[9] Kuramoto M, Kitajima N, Guo H, Furushima Y, Ikeda M, Yokoyama H: Two-photon fluorescence bioimaging with an all-semiconductor laser picosecond pulse source. Optics Letters. 2007;32(18):2726-8.

[10] Nakamura S, Masayuki Senoh, Nagahama S-i, Iwasa N, Yamada T, Matsushita T, et al.: InGaN/GaN/AlGaN-based laser diodes with modulation-doped strained-layer superlattices. Japanese Journal of Applied Physics. 1997;36:L1568.

[11] Obata T, Kitajima N, Ohta M, Ichinokura H, Masaru K: Low noise characteristics of AlGaInN-based self-pulsating laser diodes. Physica Status Solidi (A). 2008;205:1096.

[12] Ohno T, Ito S, Kawakami T, Taneya M: Self-pulsation in InGaN laser diodes with saturable absorber layers. Applied Physics Letters. 2003;83:1098.

[13] Tronciu VZ, Yamada M, Abram RA: Analysis of the dynamics of a blue-violet inxGa1-xN laser with a saturable absorber. American Physical Society. 2004;70(22): 026604-1-6.

[14] Tronciu VZ, Yamada M, Ohno T, Ito S, Kawakami T, Taneya M: Self-pulsation in an InGaN laser-theory and experiment. IEEE Journal of Quantum Electronics. 2003;39(12):1509-14.

[15] Kono S, Oki T, Miyajima T, Ikeda M, Yokoyama H: 12W peak-power 10ps duration optical pulse generation by gain switching of a single-transverse-mode GaInN blue laser diode. Applied Physics Letters. 2008;93(13):131113.

[16] Kuramoto M, Oki T, Sugahara T, Kono S, Ikeda M, Yokoyama H: Enormously highpeak-power optical pulse generation from a single-transverse-mode GaInN blue-violet laser diode. Applied Physics Letters. 2010;96(5):051102. 
[17] Oki T, Kono S, Kuramoto M, Ikeda M, Yokoyama H: Generation of over 10-W peakpower picosecond pulses by a gain-switched AlGaInN-based self-pulsating laser diode. Applied Physics Express. 2009;2:032101.

[18] Miyajima T, Watanabe H, Ikeda M, Yokoyama H: Picosecond optical pulse generation from self-pulsating bisectional GaN-based blue-violet laser diodes. Applied Physics Letters. 2009;94(16):161103.

[19] Scheibenzuber WG, Hornuss C, Schwarz UT, Sulmoni L, Dorsaz J, Carlin J-F, et al.: Self-pulsation at zero absorber bias in GaN-based multisection laser diodes. Applied Physics Express. 2011;4(6):062702.

[20] Watanabe H, Miyajima T, Kuramoto M, Ikeda M, Yokoyama H: 10-W peak-power picosecond optical pulse generation from a triple section blue-violet self-pulsating laser diode. Applied Physics Express. 2010;3(5):052701.

[21] Oki T, Koda R, Kono S, Miyajima T, Watanabe H, Kuramoto M, et al.: Direct generation of $20 \mathrm{~W}$ peak power picosecond optical pulses from an external-cavity modelocked GaInN laser diode incorporating a flared waveguide. Applied Physics Letters. 2011;99(11):111105.

[22] Olle VF, Vasil'ev PP, Wonfor A, Penty RV, White IH: Ultrashort superradiant pulse generation from a GaN/InGaN heterostructure. Opt Express. 2012;20(7):7035-9.

[23] Watanabe H, Kuramoto M, Kono S, Ikeda M, Yokoyama H: Blue-violet bow-tie selfpulsating laser diode with a peak power of $20 \mathrm{~W}$ and a pulse energy of $310 \mathrm{pJ}$. Applied Physics Express. 2010;3(12):122103.

[24] Holc K, Weig T, Köhler K, Wagner J, Schwarz UT: Impact of band structure and absorber dynamics on self-Q-switching in GaN-based multisection laser diodes at high reverse bias. Applied Physics Express. 2013;6(8):084101.

[25] Saito K, Watanabe H, Miyajima T, Ikeda M, Yokoyama H: Mode locking of an external-cavity bisection GaInN blue-violet laser diode producing 3 ps duration optical pulses. Applied Physics Letters. 2010;96(3):031112.

[26] Oki T, Saito K, Watanabe H, Miyajima T, Kuramoto M, Ikeda M, et al.: Passive and hybrid mode-locking of an external-cavity GaInN laser diode incorporating a strong saturable absorber. Applied Physics Express. 2010;3(3):032104.

[27] Kono S, Watanabe H, Koda R, Miyajima T, Kuramoto M: 200-fs pulse generation from a GaInN semiconductor laser diode passively mode-locked in a dispersioncompensated external cavity. Applied Physics Letters. 2012;101(8):081121.

[28] Koda R, Oki T, Miyajima T, Watanabe H, Kuramoto M, Ikeda M, et al.: 100 W peakpower $1 \mathrm{GHz}$ repetition picoseconds optical pulse generation using blue-violet GaInN diode laser mode-locked oscillator and optical amplifier. Applied Physics Letters. 2010;97(2):021101. 
[29] Koda R, Oki T, Kono S, Miyajima T, Watanabe H, Kuramoto M, et al.: 300 W peak power picosecond optical pulse generation by blue-violet GaInN mode-locked laser diode and semiconductor optical amplifier. Applied Physics Express. 2012;5(2): 022702 .

[30] Koda R, Takiguchi Y, Kono S, Watanabe H, Hanzawa Y, Nakajima H, et al.: Generation of a $2.2 \mathrm{~nJ}$ picosecond optical pulse with blue-violet wavelength using a GaInN master oscillator power amplifier. Submitted to Applied Physics Letters. 2015.

[31] Koda R, Oki T, Miyajima T, Watanabe H, Kuramoto M, Ikeda M, et al., editors. Generation and amplification of $400 \mathrm{~nm}$ band picosecond optical pulses by GaInN laser diodes. International Conference on Ultrafast Phenomena; 2010 2010/07/18; Snowmass Village, Colorado: Optical Society of America.

[32] Coldren LA, Corzine SW. Diode Lasers and Photonic Integrated Circuits. Wiley; 1995.

[33] Piprek J. Nitride Semiconductor Devices: Principles and Simulation: Wiley; 2007.

[34] Agrawal GP, Olsson NA: Self-phase modulation and spectral broadening of optical pulses in semiconductor laser amplifiers. IEEE Journal of Quantum Electronics. 1989;25(11):2297-306.

[35] Baveja PP, Maywar DN, Kaplan AM, Agrawal GP: Self-phase modulation in semiconductor optical amplifiers: impact of amplified spontaneous emission. IEEE Journal of Quantum Electronics. 2010;46(9):1396-403.

[36] Brosson P: Analytical model of a semiconductor optical amplifier. Journal of Lightwave Technology. 1994;12(1):49-54.

[37] Lee CH, Delfyett PJ: Limits on amplification of picosecond pulses by using semiconductor laser traveling-wave amplifiers. IEEE Journal of Quantum Electronics. 1991;27(5):1110-4.

[38] Kono S, Oki T, Kuramoto M, Ikeda M, Yokoyama H: Intensity autocorrelation measurement of $400 \mathrm{~nm}$ picosecond optical pulses from a GaInN mode-locked semiconductor laser diode using surface second harmonic generation of $\beta$-BaB2O4 crystal. Applied Physics Express. 2010;3(12):122701.

[39] Chichkov BN: Femtosecond, picosecond and nanosecond laser ablation of solids. Applied Physics A. 1996;63(2):109-15.

[40] Kauf M, Patel R, Bovatsek J, Gries W: High power UV q-switched and mode-locked laser comparisons for industrial processing applications. Proceedings of SPIE. 2008;6871:687123-10.

[41] Gu M, Li X: The road to multi-dimensional bit-by-bit optical data storage. Optics and Photonics News. 2010;21(7):28-33. 
[42] Parthenopoulos DA, Rentzepis PM: Three-dimensional optical storage memory. Science. 1989;245(4920):843-5.

[43] Strickler JH, Webb WW: Three-dimensional optical data storage in refractive media by two-photon point excitation. Optics Letters. 1991;16(22):1780-2.

[44] Walker E, Dvornikov A, Coblentz K, Rentzepis P: Terabyte recorded in two-photon 3D disk. Applied Optics. 2008;47(22):4133-9.

[45] Tashiro S, Takemoto Y, Yamatsu H, Miura T, Fujita G, Iwamura T, et al.: Volumetric optical recording using a $400 \mathrm{~nm}$ all-semiconductor picosecond laser. Applied Physics Express. 2010;3(10):102501.

[46] Tashiro S, Yamatsu H, Takemoto Y, Fujita G, Miura T, Iwamura T, et al.: Volumetric optical recording with void marks using an all-semiconductor picosecond laser. Japanese Journal of Applied Physics. 2011;50(9):09MF3. 

Chapter 3

\title{
Semiconductor Optical Amplifier for Next Generation of High Data Rate Optical Packet-Switched Networks
}

\author{
Guilhem de Valicourt, Miquel Angel Mestre Adrover, Nikolay D. Moroz and \\ Yvan Pointurier
}

Additional information is available at the end of the chapter

http://dx.doi.org/10.5772/61990

\begin{abstract}
This chapter provides an overview of considerations for the development of semiconductor optical amplifiers (SOA) for the next generations of packet-switched optical networks. SOA devices are suitable candidates in order to realize highperformance optical gates due to their high extinction ratio and fast switching time. However such devices also introduce linear and nonlinear noise. The impact of SOA devices on several modulation formats via theoretical model, numerical simulation, and experimental validation is studied. Impairments introduced by SOAs are considered in order to derive some general network design rules.
\end{abstract}

Keywords: Semiconductor optical amplifier, optical packet-switched network, coherent communications, network design rules

\section{Introduction}

Since 2010, coherent technology has paved the way toward high-capacity optical communications [1]. Coherent systems allow transmitting information with multi-level modulation formats over both polarizations [polarization division multiplexing (PDM)] of light, which increases the spectral efficiency $[\mathrm{bit} / \mathrm{s} / \mathrm{Hz}(\mathrm{b} / \mathrm{s} / \mathrm{Hz})]$ while relaxing the need for ultrahigh symbol rates to achieve data rates beyond $100 \mathrm{~Gb} / \mathrm{s}$. Such technology is enabled by fast analogto-digital and digital-to-analog converters (ADC and DAC), which, together with coherent receivers and robust digital signal processing (DSP), allow for the generation and recovery of advanced modulation formats. PDM-quadrature phase-shift keying (QPSK) and PDM-16quadrature amplitude modulation (QAM) signaling at 28 or 32 Gbaud are currently used to develop 100 and $200 \mathrm{~Gb} / \mathrm{s}$ transponders used in today's core networks. However, experimental 
results showing interfaces transmitting beyond $400 \mathrm{~Gb} / \mathrm{s}$ [2] and up to $1 \mathrm{~Tb} / \mathrm{s}$ [3] have already been demonstrated. At present, coherent systems are mainly present in long haul systems. However, a huge traffic growth (up to 560\% in 5 years) is expected by 2017 in metropolitan optical networks [4]. In addition, data centers' global traffic presents a relentless growth, with $75 \%$ of such traffic flowing within data centers. The overall need of capacity together with the drop expected in coherent transponders' cost makes evident the expansion of coherent systems toward other network segments.

Furthermore, the ever-emerging bandwidth-on-demand services in optical fiber networks induce distributed and bursty traffic profiles. Network flexibility and high efficiency are then required to handle these new traffic characteristics. Hybrid time/wavelength division multiplexing (TDM/WDM) systems could be the right trade-off between the high capacity provided by the WDM virtual point-to-point links and the TDM sub-wavelength switching granularity, allowing very efficient systems. Therefore, the WDM optical packet-switched network was proposed to unite these benefits and reduce the power consumption compared to circuit-based networks [5]. Ring nodes collect and aggregate the traffic from the access segment, into fixedduration optical packets and relay them to a WDM synchronous optical packet-ring network. Asynchronous and variable-size internet protocol (IP) packets that can be as small as 40 bytes are too short at high data rates $(10 \mathrm{~Gb} / \mathrm{s}$ in access networks, $100 \mathrm{~Gb} / \mathrm{s}$ and above in metro/core transport networks) to be efficiently switched with current optical technologies. To resolve this problem, packets are first aggregated at the network edges into "bursts" (here, slots) of data, which are sufficiently long to be compatible with the switching times of optical elements. Furthermore, synchronous switching of continuous streams of optical slots is preferred because it allows straightforward reuse of conventional erbium-doped fiber amplifiers (EDFAs).

These ring nodes also provide optical transparency for the optical slots in transit and add/drop/ blocking capabilities at the slot granularity thanks to optical logic gates or slot blocker (SB) devices [6]. SB devices are advantageous for increasing network efficiency, by erasing or transmitting the upcoming optical slots, enabling spatial reuse of the wavelengths. A slot blocker structure consists of a wavelength demultiplexer, one optical gate per wavelength enabling the structure to selectively erase any slot, and a wavelength multiplexer. The optical gate is one of the most crucial elements of the node as it is responsible for the (optical) quality of the transiting slots, and optical gate specifications, such as high extinction ratios above 25 $\mathrm{dB}$, large optical bandwidth (over the C-band), and switching times between pass and block states below $30 \mathrm{~ns}$ are typically required. Colorless optical gates, such as Mach-Zehnder modulators (MZM), electro-absorption modulators (EAM), variable optical attenuators (VOA), or semiconductor optical amplifiers (SOA), are considered as potential candidates for the SB structure. Providing a cost-efficient approach, such devices have been proposed in the silicon platform as key building blocks for the fast SB as MZM [7], ring resonator structure [8], or VOA based on $\mathrm{p}-\mathrm{i}-\mathrm{n}$ carrier-injection structures [9]. However, MZM or ring resonators exhibit low extinction ratios $(<15 \mathrm{~dB})$, like the previous type of VOA when utilized for short switching time $(<15 \mathrm{~ns})$. More recently, silicon-on-insulator (SOI)-based monolithically integrated slot-blocker integrating the multiplexer and demultiplexer as well as all optical gates was proposed in a two-port/one-port package and for single/dual polarization [10-11]. 
However, active devices are expected to offer higher extinction ratio and faster switching time along with a reduction of the insertion loss, thus avoiding the use of extra optical amplifiers as well as allowing fast power equalization providing the guarantee of error-free transmission over a large cascade of optical nodes. EAMs have also been investigated as they provide fast switching time. However, the wavelength-dependent optical losses limit the spectral operation range, and more complex structures are needed, which require the integration of EAM and SOA into a single chip [12]. The large optical bandwidth, high extinction ratio, high gain, and fast switching time make SOA more suitable candidates. Despite the development of SOA devices having a sluggish start compared to that of fiber amplifiers for in-line amplification, the recent progress in optical semiconductor fabrication techniques and device design has pushed the performance of SOAs well beyond the state of the art. SOAs promise to take a key role in the evolution of optical packet-switched networks. Furthermore, in order to drive down the cost of the SB, several approaches derived from the SOA technology have been proposed, for example, using low-cost optical gate, such as reflective SOA (RSOA) devices, designed for access network [13] or hybrid III-V on silicon optical gate array [14]. However, SOA devices, being suitable candidates as optical gate, also bring limitations on optical signal reach. The addition of amplified spontaneous emission (ASE) noise degrades the optical signal-to-noise ratio (OSNR). Their nonlinear behavior causes self-phase modulation (SPM) (when operating with single channel) translating in nonlinear phase noise. Both effects degrade signal quality and increase the bit error rate (BER). Such impairments might become critical in optical networks and even more in coherent systems where information is transmitted in both amplitude and phase of the optical signal. Therefore, novel network design rules need to be considered when introducing SOA-based optical nodes.

This chapter discusses the general context of slot-switched network with possible slot blocker implementation in Section 2 and focuses on SOA devices in Section 3. First, we present the evolution of SOA with the development of optical communication systems. The level of knowledge and development on this technology as well as a comprehensive introduction of SOA devices are described. Then, we study the impact of SOA devices of optical signals carrying data information. To do so, the impact on different modulation formats, such as onoff keying (OOK), QPSK and QAM, is explained and modeled via analytic and numerical simulations. Finally, SOA-based network design focusing on specific issues related to the linear and nonlinear noise from the SOA is presented in Section 4 . We evaluate the final performance of a large cascade of SOAs and assess novel design rules based on the accumulation of nonlinear distortions.

\section{Toward Optical Packet-Switched Networks}

\subsection{Advantages of Packet Switching}

\subsubsection{Optical circuit switching}

Optical circuit switching (OCS) has become the de facto basic standard technology underlying backbone networks since the early 2000s. With OCS, a permanent connection called "light- 
path" is statically allocated from end to end. The connection is mapped to one or several wavelengths, which are dedicated to the connection. Optical circuits can traverse nodes called optical cross-connects (OXC) or reconfigurable optical add-drop multiplexers (ROADM) "transparently," that is, without costly and energy-consuming conversions between the optical and the electrical domains, and without processing in the electrical domain, such as electronic switching. Typically, OCS networks are dimensioned for the peak demand for each connection; when demands are relatively constant with time, as is the case in backbone networks where many demands are aggregated, such provisioning is efficient and wastes little capacity. In addition, OCS suffers from the so-called $N^{2}$ scalability issue: in a network of $N$ nodes where demands are highly meshed and all or most nodes need to interact with all or most of the other nodes, the required number of circuits grows as $N^{2}$, which can quickly exhaust the limited number of available wavelengths (typically, 80-96) available in the transmission C-band on an optical fiber. OCS networks are static; they are not meant to be reconfigured faster than the second or millisecond timescale, and are typically reconfigured at much lower speeds.

For instance, Figure 1(a) depicts a six-node network with channel data rate of $100 \mathrm{~Gb} / \mathrm{s}$. Nodes consist of transponders (transmitters, TX; and receivers, RX) statically mapped to predetermined colors and an optical switching fabric that is slow reconfigurable, such that the network itself is slow reconfigurable, as mentioned above. All four (peak) demands, $75 \mathrm{~Gb} / \mathrm{s}$ from A to C, $25 \mathrm{~Gb} / \mathrm{s}$ from A to D, $100 \mathrm{~Gb} / \mathrm{s}$ from A to E, and $50 \mathrm{~Gb} / \mathrm{s}$ from $\mathrm{B}$ to $\mathrm{D}$, are routed in the optical domain, with no optoelectronic conversion. As three demands originate from node $\mathrm{A}$, three transponders are needed at node A. A full $100 \mathrm{~Gb} / \mathrm{s}$ wavelength is dedicated to the demand between $A$ and $D$, meaning that $75 \%$ of the allocated capacity is wasted for this $25 \mathrm{~Gb} / \mathrm{s} \mathrm{demand}$. Due to this waste, two wavelengths are needed between A and B (and also between B and D). It was observed that, due to optical transparency, the demand between $\mathrm{A}$ and $\mathrm{E}$ transits transparently through intermediate node $\mathrm{F}$, which only requires an optical switching fabric, but no transponder.

\subsubsection{Electronic packet switching}

In segments closer to the end user, such as metro networks or data centers, demands between the users may not be constant, and allocating circuits for the peak demand becomes inefficient and wastes network capacity. In such segments, electronic packet switching (e.g., Ethernet) is used to maximize the utilization of the networking equipment. Electronic networks are fast reconfigurable: a nanosecond-long packet may be switched from one port to another, and the next nanosecond-long packet may be switched from the same port to another port. Such reconfiguration speed is an enabler for statistical multiplexing, whereby several connections time-share the same physical equipment, such as the transponders and the optical fibers, in order to decrease the amount of deployed equipment. However, with electronic packet switching, all data arriving at a node need to be electronically converted and processed (switched); those operations are both costly and energy consuming when performed at high data rates, such as $100 \mathrm{~Gb} / \mathrm{s}$ and more per node degree. In addition, the notion of transparency is lost; upgrading a network to a higher data rate requires the upgrade of many or all network elements, while plain OCS only requires the upgrade of the end elements (the transponders) 
and not of the ROADM switching elements. For instance, in Figure 1(b), demands A-C and A-D are aggregated into a single data stream at $100 \mathrm{~Gb} / \mathrm{s}$ sharing the same wavelength due to an electronic switching fabric. Hence, node A requires only two transponders, compared with three transponders in the OCS case. However, the nodes are not equipped with optical switching fabrics and all signals sustain optoelectronic conversions and electronic processing. Demand A-E goes through an electronic switching fabric at node F, requiring an extra transponder at node F compared with the OCS case. Due to statistical multiplexing, a single wavelength is needed between nodes $\mathrm{A}$ and $\mathrm{B}$ and between nodes $\mathrm{B}$ and $\mathrm{D}$.
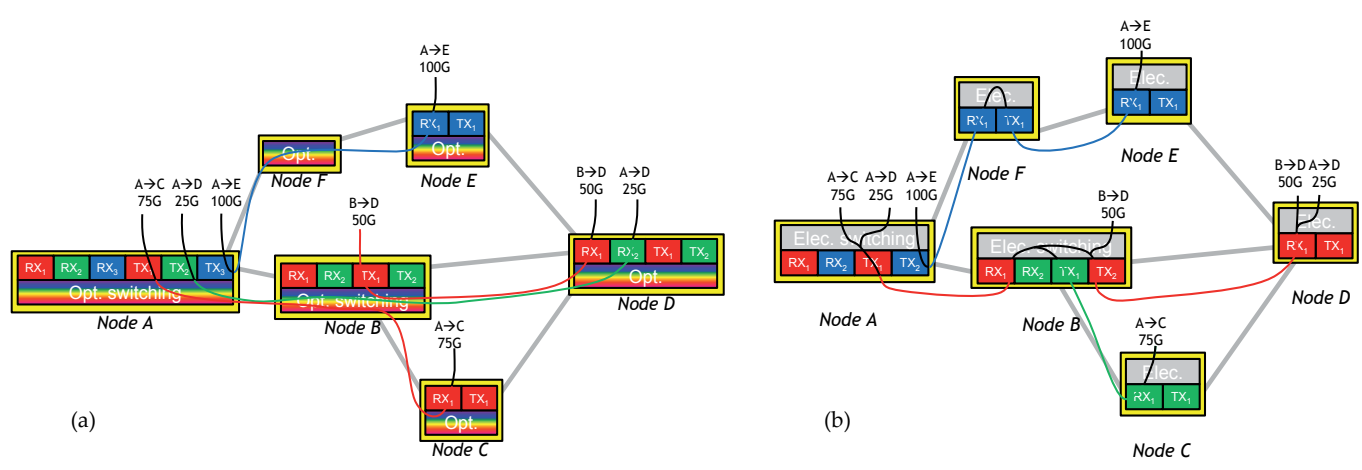

Figure 1. (a) Optical circuit switching (OCS) network. (b) Electronic packet switching (e.g., Ethernet) network.

\subsubsection{Optical transport network (OTN)}

Recently, the optical transport network (OTN), a transport technology, was introduced to enable sub-wavelength switching in OCS networks. With OTN, the same transponder may be used to send data to several destinations in a TDM fashion, thus enabling statistical multiplexing. At intermediate nodes, similar to standard electronic switching, OTN signals may be regroomed in the electrical domain in order to further optimize the packing of the data on the optical fiber. This requires a dedicated switching fabric, and breaks the optical transparency paradigm for the groomed traffic, similarly to electronic packet switching. In OTN, switching in the optical domain is done with the same slow-reconfigurable switching fabric as that of OCS, making the OTN network less dynamic than the electronic packet-switching network. For instance, in Figure 2, the A-E demand transits transparently through node F, as with OCS. Demands A-C and A-D are groomed together at node A, as in the electronic packet-switching case. As with electronic packet switching, a single wavelength is needed between nodes $A$ and $B$ and between nodes B and D.

\subsubsection{Optical packet/slot switching}

Optical packet switching (OPS) has been thoroughly investigated in the past two decades. Optical packet switching combines the benefits associated with both fast reconfigurability and dynamics (hence also statistical multiplexing and high-network utilization even under highly 


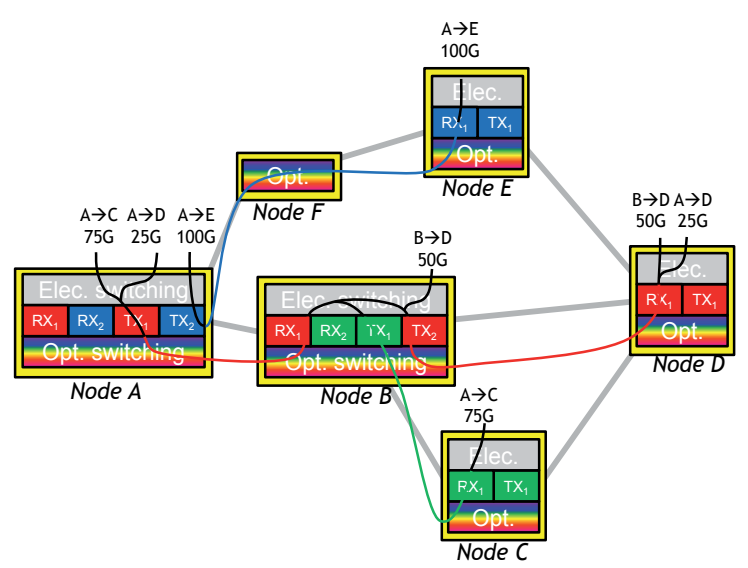

Figure 2. Optical transport network (OTN).

time-varying traffic demands) and optical transparency. With OPS, data are switched at the packet or sub-wavelength granularity directly in the optical domain with no electronic conversion or processing. For instance, in Figure 3(a), all switching is performed in an optical switching fabric at every node, and no electronic switching is required. Demands are groomed, as in the electronic packet switching or the OTN case; however, grooming is performed without optoelectronic conversions; signals may transit transparently through intermediate nodes, as with OCS or OTN. Usually, transmitters or receivers (or both) are fast wavelength tunable, that is, they can switch wavelength between the emission or reception of two consecutive packets. Optical slot switching (OSS) is a version of optical packet switching, where all switched entities are slots of the same duration. While a highly promising technology in terms of network capacity efficiency, energy consumption and flexibility [15], and despite attempts by start-up companies Matisse Networks in the late 2000s and Intune Networks in the early 2010s [16], OPS/OSS has so far not been successfully commercially deployed - essentially due to the lack of the maturity of the target markets (metropolitan and cloud networks) and underlying technical challenges. Indeed, OPS relies on very advanced, disruptive architectures and optical components, which is a very active research topic as of 2016.

Due to their simplicity, optical rings, with nodes of degree 2 only [cf. Figure 3(b)], have been extensively studied in the context of OPS. Many implementations have been proposed for optical packet rings: data and voice integration over dense WDM (DAVID) in 2003 [17], highperformance OPS WDM metro ring network (HOPSMAN) in 2008 [18], optical packet switch and transport (OPST) [19] and packet optical add-drop multiplexers (POADM) [6] in 2009, optical burst transport network (OBTN) [20] in 2011, coherent optical packet ring (COPR) [21] in 2014. Most of these proposals rely on the same component (used under various names), which is denoted here as "packet blocker." This component is able to block selected wavelengths only - the other wavelengths are allowed to cross the component transparently - at the packet granularity. While a wavelength blocker blocks selected wavelengths statically, the packet blocker may block one or several wavelengths on demand during very short periods. 
In Figure 3(b), WPB denotes either a wavelength or a packet blocker. The optical packetswitching nodes in the networks cited above typically use a combination of a wavelength or packet blocker, in the drop or in the transit path, or both. Typically, at least one of the blockers is a packet blocker, making it one of the key components of optical packet networks. If the packet blocker is used in an OSS network, it is commonly called "slot blocker." In the transit path, the packet/slot (wavelength) blocker erases the packets/slots that are received or "dropped" by a node and allow insertion or "addition" of new packets/slots (resp., wavelength) on the newly freed wavelength (this is called "wavelength reuse"). When used at the reception side of a node, the packet/slot (wavelength) blocker selects which packets/slots (wavelengths) should be received. In this case, the combination of a packet/slot blocker and of a receiver de facto implements a fast-wavelength-tunable packet (or slot) receiver. With coherent systems where the received wavelengths are dictated by the wavelength of a local oscillator, a fast-wavelength-tunable packet/slot receiver can equivalently be built using a fastwavelength-tunable laser as the local oscillator, removing the need for a packet/slot blocker at reception.

Multi-degree optical packet/slot-switching networks have also been proposed, with less maturity than the simpler degree-2 ring networks, and no commercial implementation or deployment was attempted. Time-domain wavelength interleaved network (TWIN) relies on fast-wavelength-tunable lasers and fiber couplers/splitters, and avoids the utilization of fast blockers, such as the packet blocker [22]. The optical shared memory supercomputer interconnect system (OSMOSIS) project made a different choice and embedded packet blockers as key building blocks, back in 2004 [23]. The interested reader should refer to references [2425] for other noteworthy implementations of optical packet switches.
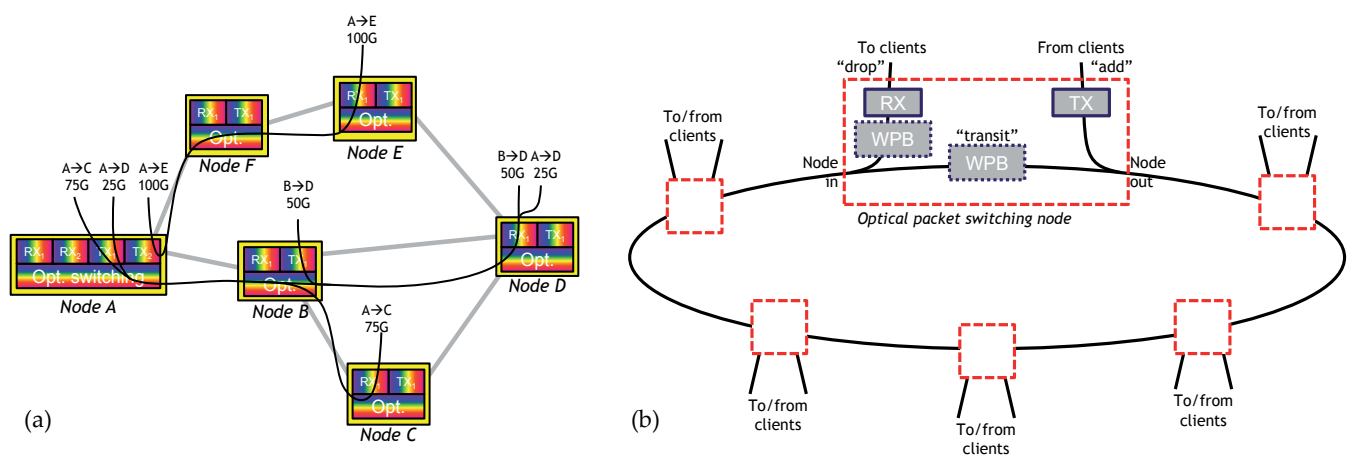

Figure 3. (a) Optical packet-switching (OPS) network. (b) OPS ring network.

\subsection{Packet/Slot Blocker Implementation}

A typical packet/slot blocker is shown in Figure 4. The wavelength-multiplexed signal is first demultiplexed, then each wavelength goes through a gate, which may block each channel independently based on control signals (not represented in Figure 4), and the wavelengths are 
multiplexed before exiting the packet/slot blocker. Due to physical limitations of the various optical components that are used to build optical switches, optical packets must be separated by a "guard interval," during which no data can be transmitted. In order to maximize the utilization of the capacity in the network, packets should be much longer (1-2 orders of magnitude) than the guard interval. With a 10-ns guard interval, packets should be at least 100 ns long, that is, 10000 bits long at $100 \mathrm{~Gb} / \mathrm{s}$. This is barely sufficient for standard 1500-byte (12000-bit) IP packets but much longer than the frequent 40-bytes (320 bits) transmission control protocol (TCP) acknowledgments contained in IP packets. Hence, optical packets usually aggregate several client (e.g., Ethernet or IP) packets. Client packet aggregation within optical packets increases network latency, which can be mitigated by reducing the optical packet length or duration. Overall, the packet length or duration should be chosen to be sufficiently large to make the guard interval negligible, yet sufficiently small to mitigate the impact of packet aggregation on network latency. This in turn means that the guard interval should be kept as small as possible, and hence that the optical gates meant to erase optical packets should be as fast as possible. This is quantified by the gate rise time (the time the gate takes to move from the passing to the blocking state) and conversely the fall time, both of which should be minimized.

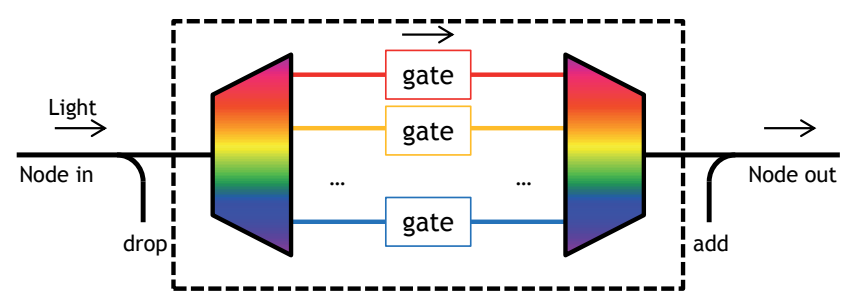

Figure 4. Typical packet/slot blocker consisting of a wavelength demultiplexer, one optical gate per wavelength, and a wavelength multiplexer. The part in the dashed box may be integrated using, for instance, silicon photonics. This "transmitting" implementation requires two connectors.

In addition, optical gates never fully erase light - a small part of the "erased" signal combines with added packets at the output of the packet blocker, causing degradation of the added signal's quality ("crosstalk"), and possibly rendering its decoding impossible. The ratio (in $\mathrm{dB}$ ) between the power of a packet before crossing a gate and its power after it is erased is called the extinction ratio. In order to minimize signal quality degradations, and thereby to maximize the reach (how many km or how many nodes a packet can cross) of a packet, the gate extinction ratio should be maximized. This is illustrated in Figure 5, where dropped packet (a) on the red wavelength is imperfectly erased by the red gate, causing crosstalk and hence signal quality degradation of packet (b), which is added on the same wavelength.

Figure 4 shows the so-called transmitting implementation of a packet blocker. All blocks within the dashed box may be integrated using one or several technologies, including silicon photonics. However, the cost of such technology is essentially driven by packaging, which largely depends on the number of connectors of the optical chip. The transmitting implementation of the packet blocker shown in Figure 4 requires two connectors. It is possible to substantially 
decrease the cost of the packet blocker by removing one connector and replacing it with mirrors, which reflect the light back to the input of the blocker, through the gates and the wavelength demultiplexer, which is then used as a multiplexer. An optical circulator is then used to separate the input and the output ports, as shown in Figure 6(a). This implementation, called a "reflective" packet blocker, additionally improves the extinction ratio as seen by the optical packets since optical packets cross twice the gates, thereby doubling the extinction ratio expressed in $\mathrm{dB}$.

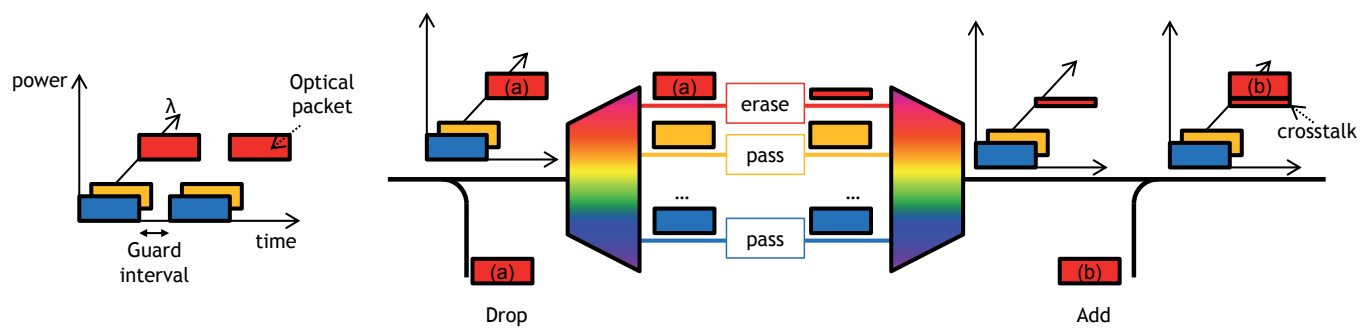

Figure 5. Crosstalk due to imperfect extinction ratio. Dropped packet (a) is imperfectly erased by the red gate; added packet (b) is combined with the leftover power (called "crosstalk") from packet (a), resulting in degraded signal quality for packet (b).

Furthermore, in today's high-capacity networks, signals are encoded on two orthogonal polarizations, transverse-electric (TE) and transverse-magnetic (TM). This doubles the capacity that can be carried by the network at little extra cost. However, some integration platforms are sensitive to signal polarization. In order to ensure compatibility of the packet blocker with dual-polarization signaling, a so-called polarization diversity scheme may be used, as illustrated in Figure 6(b); the wavelength demultiplexer/multiplexer, gates, and mirrors are all duplicated, and each set of components is used by only one polarization, for example, TM at the top of the figure (TE/TM conversion is used in order to ensure that the signal is indeed suitable for the chip) and TE at the bottom of the figure.

(a)

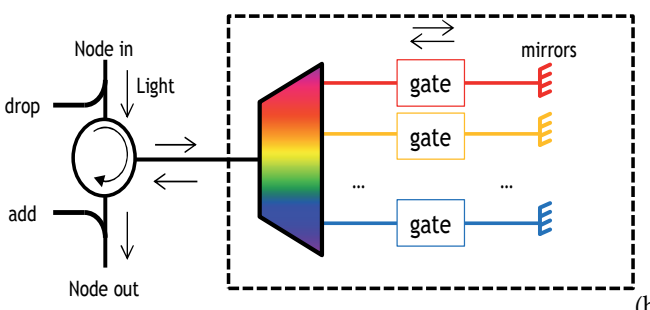

(b) 


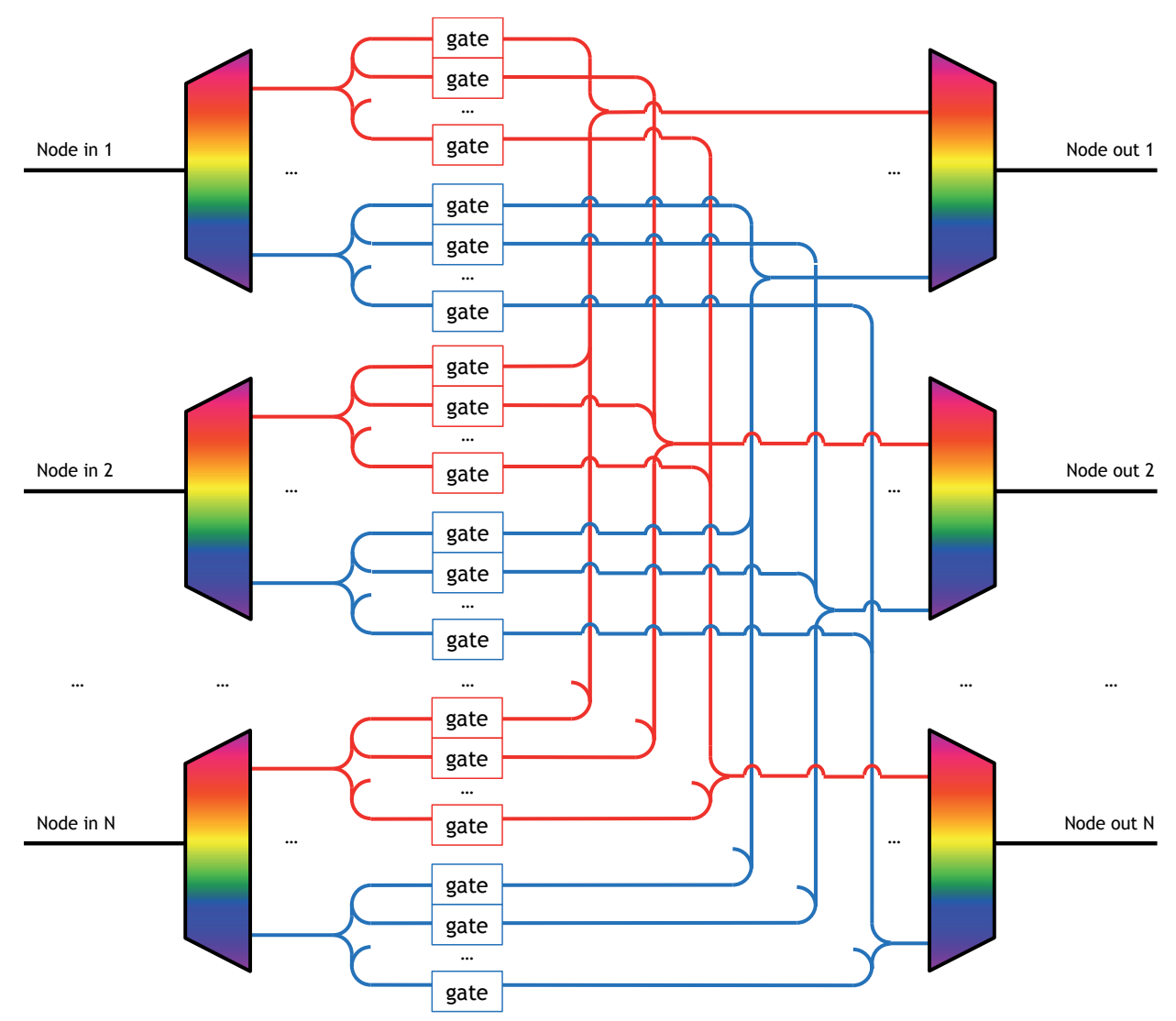

Figure 7. Sample architecture of a multi-degree $(N)$ packet blocker based on the broadcast and select technique.

The circulator enables the utilization of the waveguides between the circulator and the mirrors in both directions simultaneously. The part in the dashed box may be integrated using, for instance, silicon photonics. This "reflective" implementation requires only one connector.

It is observed that several of the recently proposed multi-degree optical packet-switching nodes make use of a so-called "broadcast and select" (B\&S) architecture [26,28], where an input signal is broadcast (through fiber splitters) to several outputs, each of which is equipped with optical gates to select which input signal will effectively select and forward to the next node. Hence, such architectures generalize packet blockers: in the multi-degree node, gates are used by each output port to select which spatial input - rather than wavelengths as in the slot blocker architecture - should be blocked or passed to the next node. Such spatial switching may further be combined with wavelength switching in more complex node architecture for increased switching capacity. A possible implementation of a multi-degree optical packet-switching fabric, which generalizes the degree- 2 packet blocker considered above, is depicted in Figure 7. Not surprisingly, the same technologies that were proposed to implement packet blocker gates can also be used to implement gates in the B\&S fabric. Specifically, SOAs are used as described in reference [26] and EAMs are used as described in 
[28]. Similarly, the same integration technologies that could theoretically be used to implement a packet blocker can also be used to implement a multi-degree switching fabric, albeit with much increased complexity stemming from the very large number of components to integrate and control, and the waveguide crossings.

\section{Semiconductor Optical Amplifier as Optical Gate}

SOA is an outstanding candidate for sub-wavelength operations in OPS or OSS. It rapidly switches between blocking and passing states to efficiently suppress or amplify optical signals. To assess the effects that this device brings into optical transmission system, the nature of signal-material interaction inside the SOA should be studied.

\subsection{Historical overview}

This section presents a historical overview of the network evolution and the semiconductor optical amplifiers. Being aware of the past component evolutions helps to understand the present state of the art and to prepare the future of optical communication. The development of semiconductor materials allowed the fabrication of high-performance devices, such as lasers and optical amplifiers. The development of SOA followed the development of laser diodes. SOAs have a gain medium as in laser devices but with the suppression of the resonance cavity. The stimulated emission concept itself was introduced by A. Einstein in 1917. The feasibility of stimulated emission in semiconductor was demonstrated in 1961 [26,27] and in 1962, the first observation of lasing action in semiconductor materials was done by several research groups $[28,29]$. The first semiconductor lasers were galliumarsenide (GaAs) homojunction devices operating at low temperature and was called Fabry-Perot laser because of the FabryPerot cavity (standard cavity with two mirrors, which are separated by an amplifying medium). Heterostructure design was proposed in 1963 [30] and demonstrated in 1969 [31]. The arrival of heterostructure devices spurred the investigation on SOAs as they were first considered at the beginning as bad lasers. In fact, high-threshold current lasers were attractive to amplify optical beam when they were operated under the lasing regime. Therefore, these first SOAs were called Fabry-Perot SOAs (FP-SOA) and have been deeply investigated [32,33]. In the 1970s, FP-SOAs were not considered seriously as important candidates for optical telecommunication [34,35], which was due to the low gain (operation below threshold current), high-gain ripple, and the instability during the amplification process because of the proximity to the threshold condition. In the 1980s, important achievements on facet reflectivity were realized and a new type of SOAs appeared. In 1982, J. C. Simon reported the first travelling wave SOA (TW-SOA) where the facet reflectivity was around $10^{-3}$ [36]. The previous drawbacks of FP-SOA were overcome. The threshold current was extremely high and allowed 15 $\mathrm{dB}$ of gain; the instability was no longer an issue at relatively high current ( $\sim 80 \mathrm{~mA})$. Development of high-quality antireflection coating was carried out and the first TW-SOA in indium gallium arsenide phosphide (InGaAsP) system with facet reflectivity of $10^{-5}$ was realized in 1986 [37]. First SOAs were based on aluminum gallium arsenides (AlGaAs), operating in the 830-nm region [38], then indium phosphide (InP)/InGaAsP SOAs appeared in the late 1980s 
[39], centered in the 1.3- $\mu \mathrm{m}$ and $1.5-\mu \mathrm{m}$ windows. In 1989, polarization-insensitive devices started to become a reality due to symmetrical waveguide structure specially designed for SOAs [40]. Prior to this, SOA structures used laser diode design (asymmetrical waveguide structure) leading to strong polarization-sensitive gain. After the invention of the EDFA, research on SOA (more specifically on TW-SOA) was slowed down for application, such as the in-line amplification, which was the main application at this time. EDFAs were chosen as the preferred solution for in-line amplification in core networks. New applications were investigated in order to take advantage of the important features of SOAs. These devices could be used as elements for all optical switches and optical cross-connects [41,42]. Highly nonlinear phenomena also take place in SOAs and can be used for wavelength conversion and crossgain modulation $[43,44,48]$. Other applications were based on SOA as intensity/phase modulators [45,46], logic gate [47], clock recovery [49, 50], dispersion compensator [51,52], format conversion from no return to zero (NRZ) to return to zero (RZ) [53], etc. The SOA is of smaller size, electrically pumped, and can be potentially less expensive than the EDFA. It can also be integrated with semiconductor lasers, modulators, etc. However, they have not been industrialized as much as EDFAs and this technology has been waiting for market needs. OPS/ OSS could be the right application, which needs unique characteristics of SOA.

\subsection{Fundamentals of SOA}

An SOA is a gain medium based on the stimulated emission. The purpose of optical amplifiers is to increase the power of the incoming signal. The input signal or light is amplified along the amplification zone. The amplification takes place in the active zone only if an external current supply is provided (considering electrically pumped device). The optical gain is associated with the stimulated emission process, which requires the recombination of electron-hole pairs. Without electrical injection, the SOA would absorb the incoming photons. RSOA is a particular scheme of SOA, which stands for reflective SOA. A mirror is inserted at the output of the device in order to reflect back light into the amplification zone. Therefore, the input and output of the device are at the same facet and the device can be more compact because of the forward and backward amplifications. SOA devices can operate in these three configurations:

1. TW-SOA using ultralow reflective end facets

2. FP-SOA where the reflections at the end facets are significant

3. RSOA where one facet is highly reflective and the other one has low reflectivity

Facet reflectivity is one of the key parameters in SOAs to form TW devices, optical cavities, or to redirect light to other directions. SOA components need a careful control on reflection from its end mirrors. Losses due to the facet reflectivity are a key parameter for mode selection. Depending on what is needed, they can be either minimized or maximized.

Gain in a semiconductor material results from current injection into the PIN structure. The relationship between the current / and the carrier density could be given by the rate equation where the carrier density is nearly uniform along the transverse dimension and neglect carrier diffusion as in reference [54]: 


$$
\frac{\partial n}{\partial t}=\frac{\mathrm{I}}{\mathrm{q} \cdot \mathrm{V}}-\frac{\mathrm{n}}{\tau_{c}}-\frac{\mathrm{g}(\mathrm{n})}{h v}|\mathrm{~A}|^{2}
$$

where $n$ is the carrier density, $I$ is the injection current, $q$ is the electron charge, $V$ is the active volume, $\tau_{\mathrm{c}}$ is the spontaneous carrier lifetime, and $h v$ is the photon energy.

The optical gain dependence on the photon energy and the carrier density is quite complex. However, it can be approximated using simple expressions:

$$
g_{m}(\lambda, n)=a \times \Delta n-a_{2}\left(\lambda-\lambda_{p}\right)^{2}
$$

where $\lambda_{\mathrm{p}}=\lambda_{0}-b_{2} \Delta n, a=\frac{\partial g}{\partial n}$ is the differential gain, $\Delta n=n-n_{0}$ is the carrier density variation, where $n_{0}$ is the carrier density at transparency, $a_{2}$ is a constant related to the spectral width of the gain spectrum, and $\lambda_{0}$ is the operating wavelength.

The use of the separate heterostructure is important in order to optimize the electronic and the optical confinement. The optical field overlaps not only the active medium but also the parts around it. The amplification takes place in the active region. Only a part of the optical field, confined in this region, takes part in the stimulated emission; therefore, the optical gain $(g)$ in equation (2) is not just equal to the material gain. The waveguide modal attenuation coefficient $\alpha_{\mathrm{in}}$, which characterizes the internal losses and the confinement factor as $\Gamma$, can be defined. These losses decrease the overall modal gain, and the net mode gain can be defined by:

$$
g_{\text {net }}=\Gamma \cdot g_{\mathrm{m}}-\alpha_{\text {in }}
$$

The internal losses can be decomposed into two different losses $\left(\alpha_{\mathrm{a}}, \alpha_{\mathrm{c}}\right)$. The modal loss in the active region is defined by $\Gamma \alpha_{\mathrm{a}}$ and the modal loss in the cladding region is defined by $(1-\Gamma) \alpha_{\mathrm{c}}$. Thus, the total internal modal loss can be written as:

$$
\alpha_{\text {in }}=\Gamma \alpha_{\mathrm{a}}+(1-\Gamma) \alpha_{\mathrm{c}}
$$

It is common practice to model gain saturation by equation (5) [54]. However, this equation ignores the distributed loss, so a new phenomenological parameter $(\varepsilon)$ is introduced to fit the gain versus power experimental curves:

$$
\frac{\ln G_{0}-\ln G(P)}{G(P)-1}=\left(\frac{P}{P_{\text {sat }}}\right)^{1+\varepsilon}
$$


where $G_{0}$ is the SOA small signal gain, $P$ is the input optical power, and $P_{\text {sat }}$ is the SOA saturation power.

In Figure $8(\mathrm{a})$, the best match for $\varepsilon$ is $\varepsilon=0.26$ in the least-mean square sense, as evidenced by the theoretical curve of Figure $8(a)$. The small signal gain is optical gain produced when SOA is operating with low-input optical signal power. Modern commercially available SOAs demonstrate fiber-to-fiber gain of $20 \mathrm{~dB}$ over the C-band with a gain peak centered around $1550 \mathrm{~nm} . P_{\text {sat }}$ is an output optical power that manifests gain decrease (saturation) by $3 \mathrm{~dB}$. Depending on technology and application field, one may currently find SOAs with 6-16 dBm of saturation power. Another important parameter is the noise figure (NF), which is the ratio between signal-to-noise ratio (SNR) before and after the SOA and it is defined as:

$$
\mathrm{NF}=\frac{\mathrm{SNR}_{\text {in }}}{\mathrm{SNR}_{\text {out }}}
$$

where $\mathrm{SNR}_{\text {in }}$ is the signal-to-noise ratio at the input of the SOA and $\mathrm{SNR}_{\text {out }}$ is the signal-tonoise ratio at the output of the SOA. Typical noise figures of commercial SOAs are in the range from $8 \mathrm{~dB}$ to $11 \mathrm{~dB}$. The optical SNR (OSNR) degradation after an optical amplifier is calculated based on the formula:

$$
\frac{1}{\mathrm{OSNR}_{\text {out }}}=\frac{1}{\mathrm{OSNR}_{\text {in }}}+\frac{\mathrm{NF} \times h v \times B_{\text {ref }}}{P_{\text {in }}}
$$

where OSNR $_{\text {in }}$ is the input OSNR, NF is the noise factor, $P_{\text {in }}$ is the input optical power, and $B_{\text {ref }}$ is the reference bandwidth $(0.1 \mathrm{~nm})$.

The spontaneous emission is created inside the active zone producing extra noise during the amplification. The spatial distribution of the carrier density is then strongly affected by this process. The spontaneous emission is produced all along (every $z$ ) the device. A part of the emitted photons are guided and amplified. They form the amplified spontaneous emission (ASE). The main noise contribution is attributed to the ASE noise [56] and OSNR degradation takes place due to the ASE noise. A given OSNR is needed to ensure specific BER values. Therefore, there is only a finite number of SOA (i.e., a finite number of spans), which could be cascaded to ensure a given BER. The output OSNR after traversing one SOA and depending on the input optical power is presented in Figure 8(b). According to equation (7), the output OSNR degrades at low input power (due to higher noise accumulation); therefore, BER increases. In order to mitigate its impact, higher input power needs to be injected into the SOA. However, as ASE noise limits minimal input optical power, the nonlinear behavior of SOA limits the maximum input optical power. Therefore, BER increases at high input power even if the OSNR is not degraded. 
(a)

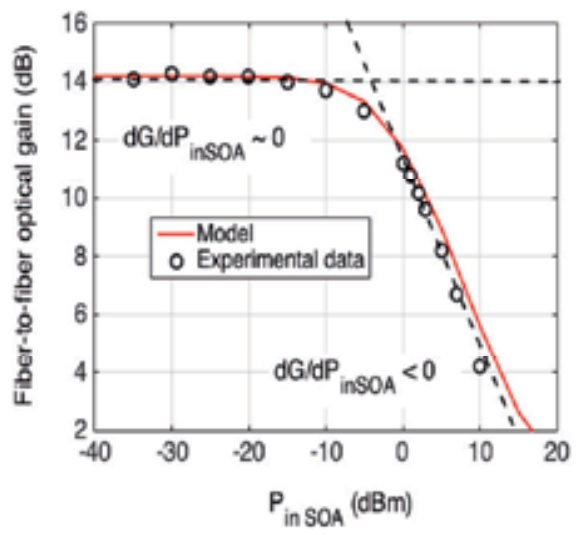

(b)

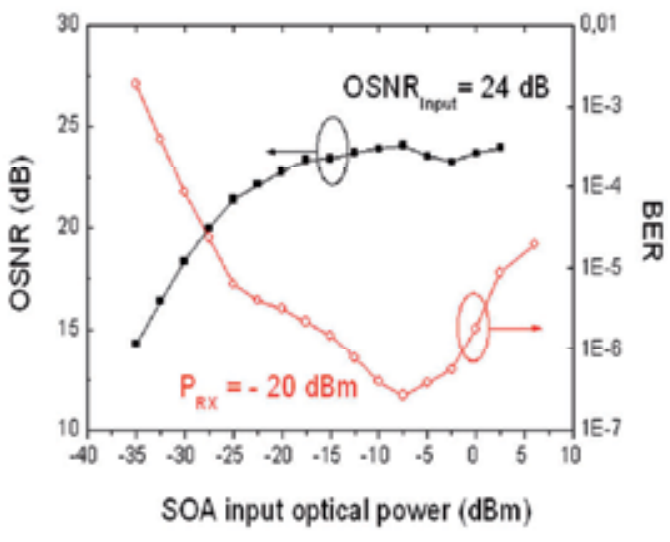

Figure 8. (a) Measured and modeled SOA optical gain. (b) BER for a 10 Gbit/s OOK-NRZ signal and OSNR measurements depending on the SOA input optical power.

We can approximate that SOA parameters responsible for nonlinear effects are spontaneous carrier lifetime $\left(\tau_{c}\right)$ and line-width enhancement factor $(\alpha)$. As SOA has the same active medium as semiconductor lasers, carrier lifetime is in the range of $200-700$ ps. The carrier lifetime is inversely proportional to the recombination rate. The photon density from the amplified signal and the spontaneous emission affects the carrier density. Since photon density is not homogeneous along the SOA, carrier density also depends on the position. This spatial hole burning (SHB) is also present in many lasers, but it results from the coupling between carriers and a single photon population. These saturation effects strongly influence the static and dynamic performances of SOAs [57,58]. A multi-section model could be considered in order to take into account such spatial distribution; more details on simulations along the SOA length can be found in reference [59] and in the following section. In this section, we will consider the carrier lifetime as constant for sake of simplicity. Factor $\alpha$ also known as Henry factor (after Charles Henry who explained the line-width enhancement phenomenon in reference [60]) is responsible for line-width broadening after the SOA and has values within the range from 4 to 7 .

The signal power and the phase are found to satisfy [54]:

$$
\begin{gathered}
\frac{\partial P}{\partial z}=g(z, \tau) P \\
\frac{\partial \Phi}{\partial z}=\alpha_{\mathrm{H}} \frac{g(z, \tau)}{2}
\end{gathered}
$$

It can be easily demonstrated by combining both equations that:

$$
\alpha_{\mathrm{H}}=2 P \frac{\partial \Phi}{\partial P}
$$


where $\frac{\partial \Phi}{\partial P}$ is the variation of the phase over the variation of the optical power.

It has been shown that SOAs can be used in order to control the chirp of an input optical pulse [61]. The chirp parameter $\alpha_{\mathrm{H}}$ is defined as in equation (9). Assuming the $\Delta n$ is homogeneous along the SOA, the gain variation (from the single-pass gain expression) can be written as:

$$
G=e^{\Gamma a \Delta n L}
$$

So the gain variation in $\mathrm{dB}$ can be written as:

$$
\Delta G_{\mathrm{dB}}=\Gamma a \Delta n L \cdot 10 \log _{10}(e)
$$

And the phase variation as:

$$
\Delta \Phi=-\frac{2 \pi}{\lambda} \cdot \Delta n_{\mathrm{ref}} L=\frac{\alpha^{\prime}}{2} \Gamma a \Delta n L
$$

where $\lambda, n_{\text {ref }}$ and $L$ are wavelength, refractive index, and SOA length, respectively. $\alpha$ ' is the line-width enhancement factor of the semiconductor material, which is defined as:

$$
\alpha^{\prime}=-\frac{4 \pi}{\lambda \Gamma a}\left(\frac{\partial n_{\mathrm{ref}}}{\partial n}\right)
$$

The alpha factor depends on the gain variation under modulated input optical power. It can be demonstrated as in reference [62]:

$$
\alpha_{\mathrm{H}}=\alpha^{\prime} \frac{d G / d P_{\text {in SOA }}}{1+d G / d P_{\text {in SOA }}}
$$

where $P_{\text {in }}$ is the SOA input optical power.

When the optical input power is low enough, equation (14) gives $\alpha_{\mathrm{H}}=0$ because the carrier density remains unchanged compared to the value at the equilibrium $(\Delta n=0)$ and $d G / d P_{\text {in }} \sim 0$. No gain variation is observed as represented in Figure 8(a), so no degradation of the optical signal should be observed even with high line-width enhancement factor $(\alpha)$. However, as the optical input power increases, carrier depletion occurs in SOA $(\Delta n<0)$ and induces gain saturation $\left(\mathrm{d} G / \mathrm{d} P_{\text {in }}<0\right)$. Since $\alpha>0$ in gain medium, such as in SOA, the chirp parameter is negative $\left(\alpha_{\mathrm{H}}<0\right)$ for the gain-saturated SOA. The frequency components of the 
leading edge of the pulse are red-shifted and trailing edge blue-shifted when the chirp is negative $\left(\alpha_{\mathrm{H}}<0\right)$ as explained in reference [61]. Therefore, a trade-off arises between nonlinear distortions (at high input power) and noise (at low input power) and an optimum input power can be defined. The impact of the line-width enhancement factor on the signal integrity (mainly adding phase noise) is studied in Section 3.3.3.

\subsection{SOA Model and Simulation}

\subsubsection{Analytical model for OOK-NRZ signal}

In this section, we propose an analytical model to predict the impact of SOA devices on an NRZ optical signal [63]. In the linear regime (before gain saturation), the NF is used to predict the OSNR degradation as explained previously. However, when the input optical power becomes higher, such formula does not apply anymore as the main degradation of the signal comes from the nonlinear behavior of the SOA. To assess the nonlinear impairments due to the SOAs, we study the evolution of the OSNR penalty for a given target BER, as a function of SOA input power and of the number of cascaded SOAs. Here, the OSNR penalty is the difference between the required OSNRs (in $\mathrm{dB}$ ) to ensure the target BER (here, $\mathrm{BER}_{\mathrm{t}}=10^{-3}$ ) after traversing SOAs and in back to back. We assume here that the distortions of the signal can be described by the extinction ratio $r$ (amplitude modulation only), defined as $r=P_{1} / P_{0}$, where $P_{1}$ and $P_{0}$ are the average powers of marks and spaces, respectively. The relationship between OSNR and BER is given as in reference [64]:

$$
\operatorname{BER}=\frac{1}{2} \operatorname{erfc}\left(\frac{\mathrm{Q}}{\sqrt{2}}\right)
$$

$\mathrm{Q}=\frac{\sqrt{\mathrm{r}}-1}{\sqrt{\mathrm{r}+1}} \sqrt{\frac{\mathrm{OSNR}_{\mathrm{ref}} \times \mathrm{B}_{\text {ref }}}{\mathrm{B}_{\mathrm{e}}}}$, where $\operatorname{erfc}($.$) is the complementary error function, \mathrm{B}_{\text {ref }}$ is the optical bandwidth for which the measured OSNR (12.5 GHz in this work) is expressed, and $\mathrm{B}_{\mathrm{e}}$ is the receiver electrical bandwidth. Thus, for a given value of $r$, the OSNR penalty Pen $(r$ ) (in $\mathrm{dB}$ scale) can be easily derived [63]:

$$
\operatorname{Pen}(r)=20 \log \left(\frac{\sqrt{r}+1}{\sqrt{r}-1} \times \frac{\sqrt{r_{0}}-1}{\sqrt{r_{0}}+1}\right)
$$

where $r_{0}$ is the extinction ratio in back to back.

We now explain how the saturation of the SOA gain can impact $r$ when an OOK-NRZ signal goes through. At low-input power levels, below $-20 \mathrm{dBm}$, in the linear regime, the gain of the SOA is independent of the input power, and we expect no impact on the extinction ratio of an incoming signal. At high power levels, the SOA turns nonlinear, and the SOA gain saturates and decreases [Figure 8(a)]. Thus, we expect a decrease of the extinction ratio of an incoming 
signal at SOA output. Optical gain saturation could be modeled as described in the previous section. Let us suppose we have $N$ identical cascaded SOAs, and that the input optical power into each SOA is fixed to $P_{\text {in }}$ employing variable optical attenuators at the SOA inputs. The extinction ratio at the output of the $k^{\text {th }}$ SOA, where $k=1, \ldots, N$, is denoted by $r_{k}$, and the average optical powers of " 1 " and " 0 " at the input of the $k^{\text {th }}$ SOA are denoted by $P_{1}^{(k)}$ and $P_{0}^{(k)}$, respectively. For $k=1, \ldots, N$, it can be written as:

$$
P_{1}^{(k)}=\frac{2 r_{k-1} P_{\text {in }}}{1+r_{k-1}} \quad \text { and } P_{0}^{(k)}=\frac{2 P_{\text {in }}}{1+r_{k-1}} \text { and } r_{k}=\frac{P_{1}^{(k)} G\left(P_{1}^{(k)}\right)}{P_{0}^{(k)} G\left(P_{0}^{(k)}\right)}
$$

With such an analytic model, we can iteratively estimate the degradation of the extinction ratio after the cascade of SOAs and deduce the OSNR penalty, regardless of the input power or the number of cascaded SOAs. The nonlinearity accumulation could then be extracted for a large number of cascaded SOA as explained in Section 4. In order to analyze the impact of SOA device on high-order modulation format, a more complex model is needed as described in the following section.

\subsubsection{SOA multi-segment model for high-order modulation format}

Coherent optical transmission systems may be deployed in metropolitan and data center networks in the near future as detailed in Section 1. These systems operate with QPSK and QAM signals that use both amplitude and phase of the optical signal to transmit information. To assess the impact of the SOA cascade on such signals, we need to use models that can simulate the evolution of carrier density along the active zone of the SOA with high precision. The computation complexity of the model should be taken into account when the task is to analyze a large cascade of the SOAs in optical transmission line via numerical simulation. As a trade-off between precision and complexity, we find that a multi-segment SOA model is a suitable solution to assess the impact of the SOA on optical signals in coherent transmission systems. Such model from reference [65] demonstrates sufficient accuracy and enables study of cascades of numerous SOAs in a reasonable time. As pointed out in reference [66], one may transform this model in two ways. First, further simplification with small perturbation approach will provide a low-complexity SOA simulator for small signal cases. Second, adding equations of $\mathrm{SHB}$ and carrier heating $(\mathrm{CH})$ augments the complexity but provides a simulator sensitive to these ultrafast phenomena. However, characteristic time of gain recovery from SHB and $\mathrm{CH}$ is less than $10 \mathrm{ps}$ as presented in reference [67], and thus they should have no impact on optical signal below $100 \mathrm{GHz}$ frequencies (our range of study). To make the model suitable for static and dynamic analysis, some assumptions have been made and simplifications have been introduced. The material gain is assumed to vary linearly with the carrier density but with no wavelength dependence as described in reference [68]. The total device length is divided into smaller sections, where the carrier density is assumed to remain constant, in order to simulate the carrier density variation. More details are presented in reference [59]. Scheme of the proposed SOA model is presented in Figure 9. We used a model with ten 
segments in our simulations. Each segment consists of three stages: amplification, ASE addition, and internal loss section. The premise to segmentation and stage separation solution was formed using the technique proposed in reference [54]. To obtain a relatively simple equation for SOA gain stage [as equation(10)], spontaneous emission processes as well as internal loss were neglected. Therefore, second (ASE) and third (loss) stages were added for completeness of the model. Moreover, to consider signal-noise interactions and loss influence on traversing signal, one should use several segments such that ASE noise and loss from previous segments will affect signal evolution in the subsequent ones.

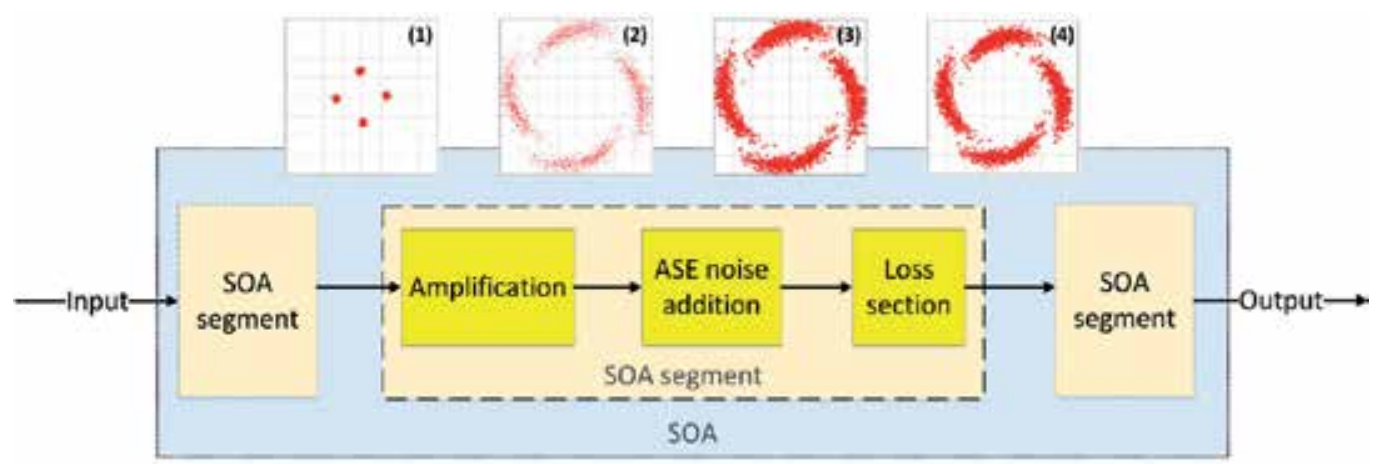

Figure 9. Scheme of SOA segmented model ([2]) with QPSK constellation evolution after each stage: constellation at the segment input (1), after amplification stage (2), after ASE addition (3), and after loss section (4).

According to reference [54], equation. (1) could be written such that the first stage of each SOA segment is an amplification stage where we calculate SOA gain due to stimulated emission from:

$$
\frac{\partial h(t)}{\partial t}=\frac{h_{0}-h(t)}{\tau_{\mathrm{c}}}-\left(e^{h(t)}-1\right) \frac{P_{\mathrm{in}}(t)}{P_{\mathrm{sat}} \tau_{\mathrm{c}}}
$$

with $h$ being the SOA gain integrated along the segment's length, $h_{0}$ small-signal gain per segment, saturation power $P_{\text {sat }}$ and incident optical power $P_{\text {in }}$. We use Runge-Kutta method (RK4) to solve this equation for signal symbol by symbol. After we obtain $h(t)$, we are ready to calculate output optical field $A_{\text {out,amp }}(t)$ after the amplification stage:

$$
A_{\text {out }, \text { amp }}(t)=A_{\text {in }}(t) e^{\frac{h(t)}{2}\left(1-j \alpha_{\mathrm{H}}\right)}
$$

where $\alpha_{\mathrm{H}}$ is the alpha factor of the SOA as described by equation (14).

The second stage is ASE addition. To calculate the ASE noise, one would also use the obtained gain values from equation (18): 


$$
A_{\mathrm{ASE}}(t)=\sqrt{\mathrm{NF}\left(e^{h(t)}-1\right) h v B_{0}}\left(n_{I}(t)+j n_{\mathrm{Q}}(t)\right)
$$

with NF denoting noise figure, $h v$ denoting signal photon energy, $B_{0}$ denoting noise integration bandwidth at the receiver, $n_{I}(t)$ and $n_{Q}(t)$ denoting independent Gaussian processes for ASE randomness simulation.

The third stage is dedicated to loss simulation. Usually, internal SOA loss is considered constant and optical field after one SOA segment may be written as follows:

$$
A_{\text {out }, \text { seg }}(t)=\left(A_{\text {out }, \text { amp }}(t)+A_{\text {ASE }}(t)\right) e^{\alpha_{\text {int }}}
$$

where $\alpha_{\text {int }}$ is SOA internal loss per segment.

\subsubsection{SOA parameters impact on signal quality}

To evaluate the impact of SOA parameters on passing optical signal, we have simulated SOAs with different parameters and calculated relative deviation. We used $2^{15}-1$ pseudorandom bit sequences (PRBS) modulated at 10.7 Gbaud in NRZ QPSK signal with initial OSNR of $40 \mathrm{~dB}$ at two input optical powers: $-30 \mathrm{dBm}$ for the small-signal regime and $+5 \mathrm{dBm}$ under gain saturation regime. Our SOA parameters were as follows: $14.2 \mathrm{~dB}$ small-signal gain, $5 \mathrm{dBm}$ saturation power, noise figure of $8 \mathrm{~dB}$, line-width enhancement factor of 5 , and carrier lifetime of 400 ps. Then, we varied one of these parameters and observed the output optical signal evolution. We calculated relative amplitude deviation $\Delta A / A_{\text {ideal }}$ (RAD) and relative phase deviation $\Delta \varphi / \varphi_{\text {ideal }}(R P D)$ based on received signal constellations as detailed in Figure 10. Our metrics are normalized in such way that RAD and RPD should be equal in the case of Gaussian white noise around the symbols.

Changing small-signal gain has little influence on generated noise while in unsaturated regime, as can be seen in Figure 11(a). Here, augmenting gain means augmenting both amplitude and phase noise. During saturation, increase in gain translates into more abrupt saturation and, therefore, larger amplitude fluctuations. Amplitude noise couples with phase noise through alpha factor; therefore, we observe that both noises grow. Saturation power determines where saturation begins to manifest. While saturation power grows, the SOA becomes a more linear amplifier (keeping the input optical signal power constant). That means less gain fluctuations and less noise as well, as demonstrated in Figure 11(b). Noise figure is proportional to the ASE noise generated within the SOA. In small-signal regime, the ASE noise is prevailing and NF increase manifests equivalently in both amplitude and phase noise, as presented in Figure 12(a). Remarkably while in saturation, nonlinear effects exceed ASE impact to such an extent that we observe no influence of the NF. Carrier lifetime is a characteristic of how fast gain reacts to input power changes. While SOA works with small input powers, its gain does not vary and carrier lifetime has no impact on amplitude and phase noises as shown in Figure 


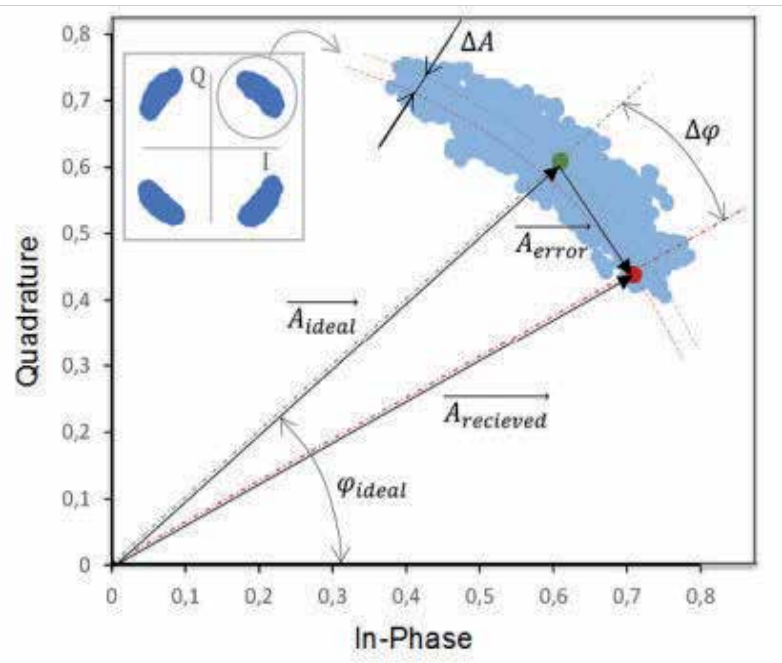

Figure 10. QPSK constellation symbol upon reception. Ideal transmitted symbol is shown with $\overrightarrow{\mathrm{A}_{\text {ideal }}}$ and illustrative received symbol - with $\overrightarrow{\mathrm{A}_{\text {received }}}$. Amplitude error $\Delta \mathrm{A}$, phase error $\Delta \varphi$ and error vector $\overrightarrow{\mathrm{A}_{\text {error }}}$ are demonstrated.

12(b). However, when carrier lifetime decreases, the SOA's gain dynamics becomes fast enough to follow shorter signal power changes producing amplitude and phase noise, the socalled bit-patterning effect. We observe the resonance when the parameter becomes equal to the inverse of signal's symbol rate $(1 / 10.7 \mathrm{GHz}=93 \mathrm{ps})$. Further decrease in carrier lifetime accelerates gain recovery, which diminishes SOA contribution to optical noise.

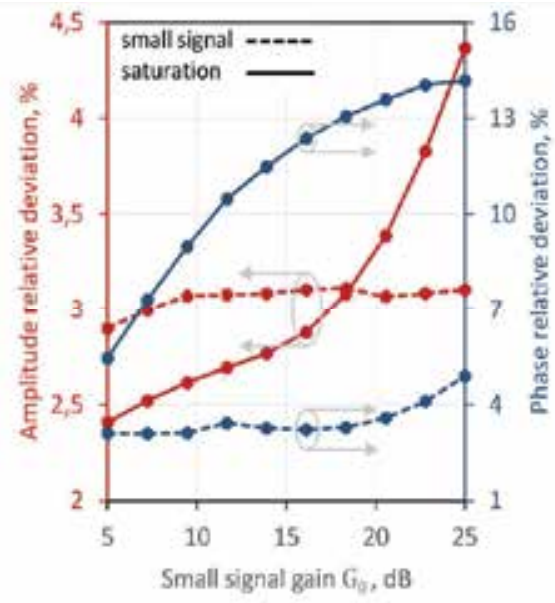

(a)

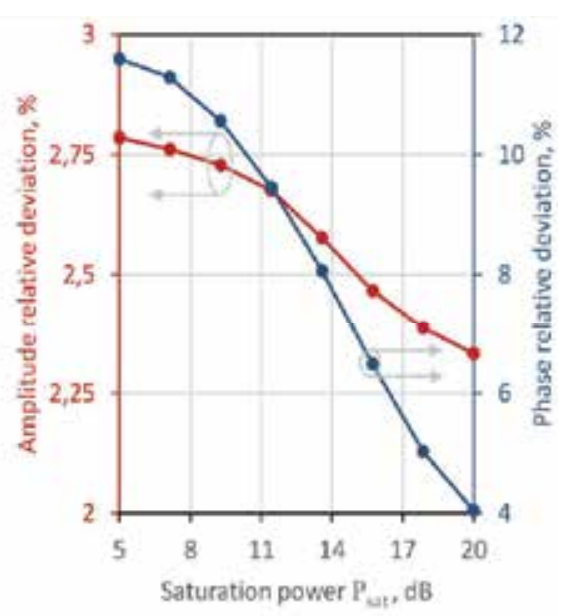

(b)

Figure 11. Relative amplitude (in red) and phase errors (in blue) for different small-signal gains (a) and saturation powers (b) of the SOA. Dashed line corresponds to small-signal mode and solid line corresponds to gain saturation mode. 


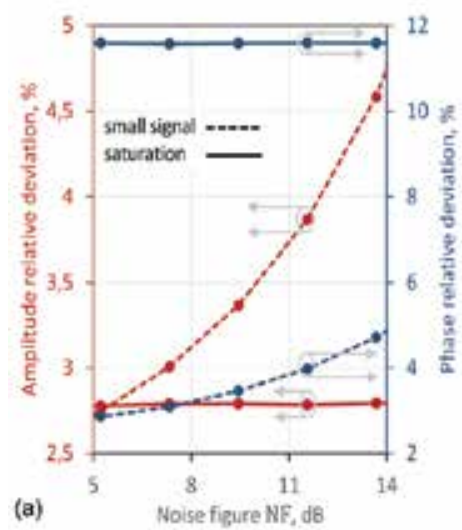

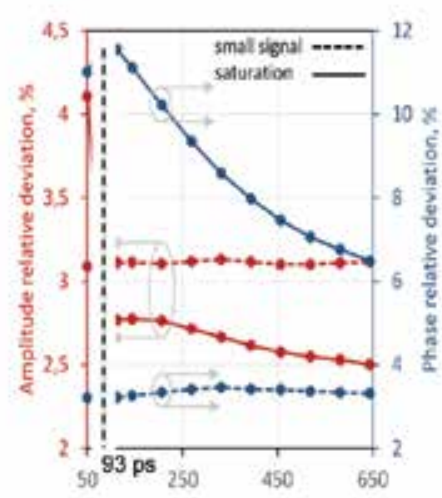

(b) Ufetime of charge carriers $\tau$, , ps

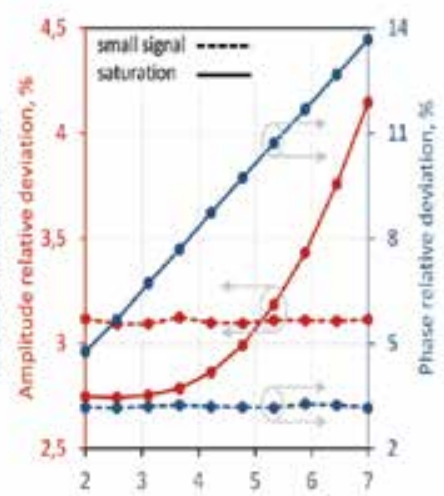

(c) Linewidth enhancement factor a'

Figure 12. Relative amplitude (in red) and phase errors (in blue) for different noise figures (a), for different charge carriers lifetimes (b), and line-width enhancement factors of the SOA (c). Dashed line corresponds to small-signal mode and solid line corresponds to gain saturation mode.

Factor $\alpha^{\prime}$ translates the input power fluctuation into gain changes and phase changes. No gain change in small-signal regime is observed for any input optical power [as predicted in Figure 8(a)]; therefore, $\alpha$ ' does not impact the quality of the signal as demonstrated in Figure 12(c). In the saturation regime, $\alpha^{\prime}$ determines the nonlinear phase noise of the SOA and as $\alpha^{\prime}$ grows, phase noise increases.

\section{Cascade of SOA}

In optical packet-switched network, the optical packet could travel across a large number of nodes. Therefore, the technology used for the logic gate strongly impacts the system performance and could limit the operating power range. Thus, an estimate of the OSNR penalties is needed to design a complete system, as described in the next section.

\subsection{Design Rules for SOA-based Optical Network}

\subsubsection{Direct detection technology: on-off keying}

An analytical model was proposed in Section 3.3.1. Such model predicts the OSNR penalty due to the extinction ratio degradation as the signal goes through a series of SOAs. Such degradation is defined by the optical gain profile as presented in Figure 8(a) and translated to a BER degradation, as observed in Figure 8(b). In Figure 13(a), the modeled OSNR penalty is plotted against the product between the SOA average input power $P_{\text {in SOA }}$ and the number $N$ of cascaded SOAs (in $\mathrm{dB}$ scale, i.e., $10 \log _{10}\left[N \times P_{\text {insOA }}(\mathrm{dBm})\right]$ ), versus input powers between $[-30$ $\mathrm{dBm} ;+7 \mathrm{dBm}]$ and up to 50 cascaded SOAs. The SOA parameters are the same as detailed in reference [63]. We observe that all points gather almost on a single curve. Hence, it is tempting 
to approximate the penalty as a function of the product $N \times P_{\text {inSOA }}$. Such a behavior is similar to that observed in the cascade of fiber spans in dispersion-managed systems under the influence of Kerr nonlinear effect [55]. The OSNR penalty was shown to scale as a function of the sum of the input powers into each line fiber span, (or more accurately as a function of the nonlinear cumulated phase). By analogy with reference [64], the product $N \times P_{\text {insoA }}$ will here be referred to as the integrated power (IP), which could be generally defined as:

$$
I P=\sum_{S O A k} P_{i n S O A}^{(k)}=P_{\text {in } S O A} \times N
$$

In direct detection systems with chromatic dispersion (CD) compensation, a maximum of 1.5 $\mathrm{dB}$ OSNR penalty is usually accepted for fiber-induced nonlinear penalties [64]. According to Figure 13(a), the range of possible values of integrated power leading to such threshold penalty does not exceed $1.6 \mathrm{~dB}$ with $N$ ranging from 1 to 50 sections, which is reasonably accurate. By analogy with cascades of fiber spans, we also define the nonlinear threshold (NLT) as the minimum value of integrated power leading to the threshold penalty. In order to derive general design rules, the NLT (for $1.5 \mathrm{~dB}$ of OSNR penalty) has been modeled depending on the optical gain, the saturation power as represented in Figure 13(b). Therefore, it is possible to find out the NLT as a function of the SOA design or/and the operating condition. Low optical gain and high saturation power are mandatory in order to optimize such NLT. In Section 4.2.1, after checking that the $N \times P_{\text {inSOA }}$ behavior is also observed experimentally from a more limited number of cascaded SOAs (up to 4), we will experimentally characterize the NLTs of the (R)SOA devices under study and use them to derive the maximum acceptable number of cascaded SOAs.
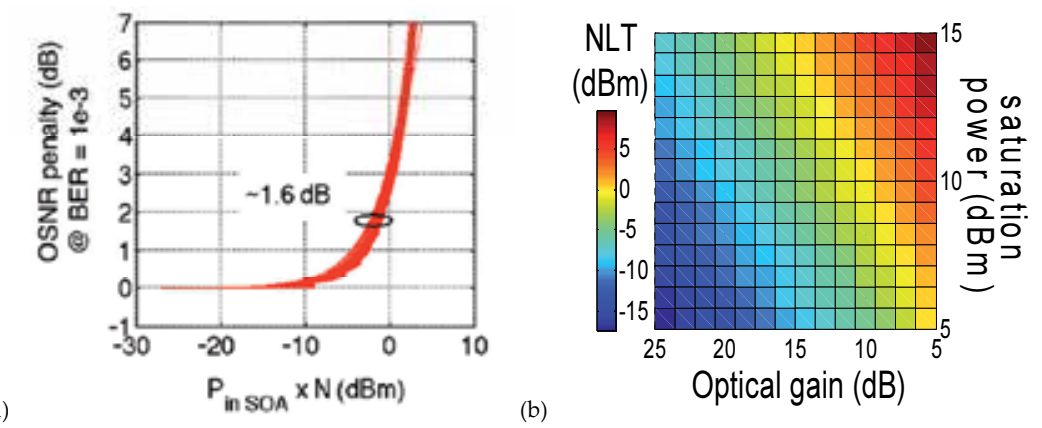

Figure 13. (a) Modeled OSNR penalty for SOA devices depending on $N \times P_{\text {in SOA }}$ for $10 \mathrm{Gbit} / \mathrm{s}$ NRZ signal and (b) NLT dependence on SOA parameters $\left(G, P_{\text {sat }}\right)$

\subsubsection{Coherent technology: advanced modulation formats}

We simulate an optical fiber line including SOA (based on the 10-section model presented in Section 3.3.2) and optical fiber with a 28-Gbaud RZ optical signal path through a cascade of SOAs. We use root-raised cosine filter with 0.1 roll-off for pulse shaping a payload of $2^{15}-1$ symbols. Compared to experimental evaluation, the simulated input optical signal has less 
optical power fluctuation; therefore, we chose an RZ signal in order to emphasize the nonlinear impact of SOA. We implement the multi-section model described in Section 3.3.2 with 10 sections. All SOA model parameters are detailed in reference [69]. Single-channel and singlepolarization QPSK, 8-QAM, and 16-QAM optical signals are launched into a cascade of SOAs. For each simulation, we vary the input power of the SOAs. We plot OSNR penalty as a function of the IP. In the case of nonlinear noise occurring in fiber networks without inline CD compensation, the threshold OSNR penalty equals $1.76 \mathrm{~dB}$ providing the best transmission quality [70]. We use the same penalty to assess the NLT after the cascade of SOAs. We investigate the convergence of OSNR penalty curves depending on the IP for different modulation formats in Figure 14(a) as described for OOK signals. The modeled OSNR penalty is plotted against the IP (in dB scale).

A maximum IP spread of $2.6 \mathrm{~dB}$ and $1.5 \mathrm{~dB}$ are obtained for QPSK and M-QAM signals, respectively (spread of $1.6 \mathrm{~dB}$ was obtained with OOK signals) [69].
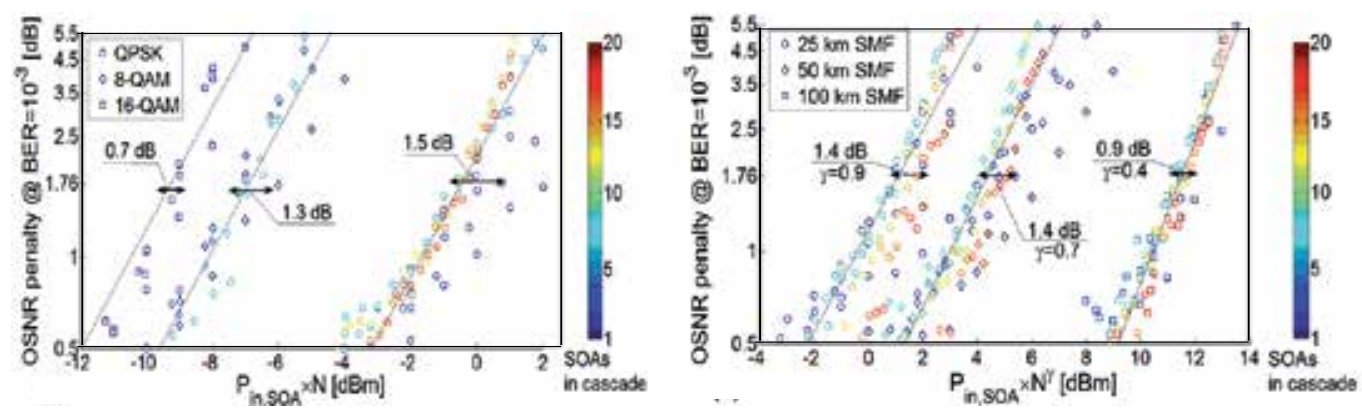

Figure 14. OSNR penalties (a) for a cascade of 20 SOAs with QPSK, 8-QAM, and 16-QAM signal and (b) for a cascade of 20 SOAs with $25 \mathrm{~km}, 50 \mathrm{~km}$, and $100 \mathrm{~km}$ of single-mode fiber (SMF) spans.

We simulate different types of spans including $25 \mathrm{~km}, 50 \mathrm{~km}$, and $100 \mathrm{~km}$ of SMF and one SOA with a 100-G QPSK signal in Figure 14(b). We have introduced a new phenomenological parameter $\gamma$ in order to take in account the changing character of the nonlinear accumulation when using extra optical fibers. Therefore, a more general law $\mathrm{IP}_{\mathrm{g}}=P_{\mathrm{in}} \times N^{\gamma}$ is introduced and presented in Figure 14(b).

A compromise between cumulated nonlinear distortions and ASE noise need to be taken in account when optimizing the number of cascaded devices. The evolution range of SOA input powers with respect to the maximum number of cascaded nodes is presented in Figure 15. This model used the nonlinear threshold of the Figure 14 to set the maximum input power into the SOAs. The OSNR degradation is calculated based on the NF of the SOA. An OSNR margin of $3 \mathrm{~dB}\left(\right.$ at $\left.\mathrm{BER}=10^{-3}\right)$ with respect to back to back is assumed for aging and implementation penalties as well as $1.5 \mathrm{~dB}$ of penalty coming from the nonlinear behavior of the SOA (worstcase estimation). Therefore, the maximum number of cascaded devices for a given input optical power is reached when the targeted OSNR is obtained. The OSNR is expected to remain above the OSNR limit and the integrated power needs to remain below the NLT. The largest count of cascadable devices is obtained at the optimal trade-off between nonlinear distortions and ASE noise. ASE noise accumulates from one device to the next. In order to mitigate its impact, 


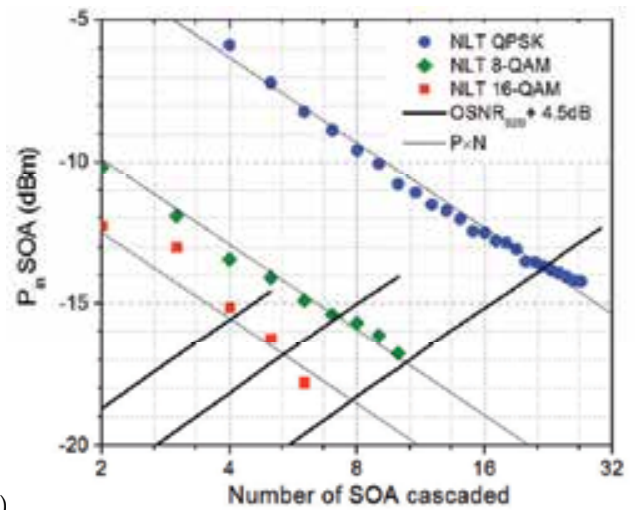

(a)

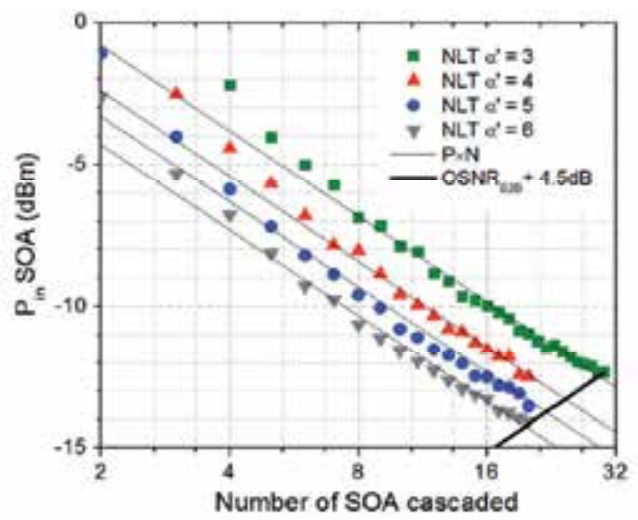

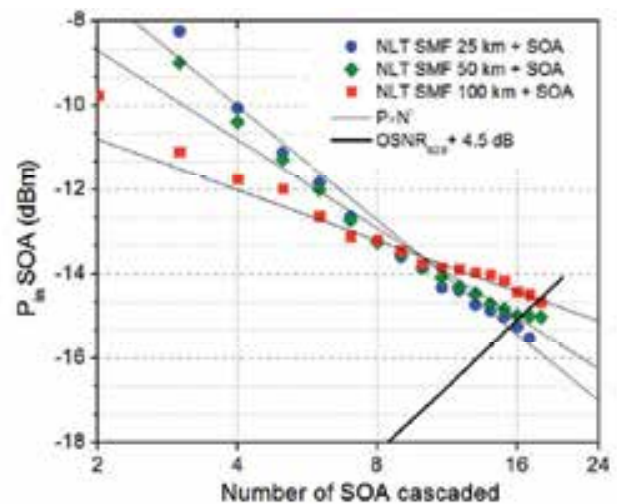

(b)

Figure 15. NLT and OSNR limits versus number of cascaded SOAs (a) for QPSK, 8-QAM, 16-QAM formats of signal, (b) for QPSK signal and $25 \mathrm{~km}(\gamma=0.9), 50 \mathrm{~km}(\gamma=0.7)$, and $100 \mathrm{~km}(\gamma=0.4)$ SMF inter-SOA spans, (c) for QPSK signal and SOA line-width factor 3, 4, 5, 6, and (d) for QPSK signal and SOA carriers' lifetime 100 ps, 200 ps, 300 ps, and 400 ps.

higher input power needs to be injected into the SOA. However, the nonlinear behavior of SOA limits the maximum input optical power. QPSK, 8-QAM, and 16-QAM optical signals are studied in Figure 15(a). The NLT slope is the same for all three modulation formats. The impact of the extinction ratio explains the large shift from QPSK to M-QAMs due to amplitude level changes (as previously explained for OOK signals). Different fiber span lengths are simulated in Figure 15(b). We use the general IP law as previously detailed. The noise dependence on span input cumulated $\mathrm{CD}$ and uncorrelated noise contributions from span to span due to the large relative span input cumulated CD [71] induces such that $\gamma$ is close to 0.67 for $100-\mathrm{km}$-long CD-unmanaged $100 \mathrm{~Gb} / \mathrm{s}$ systems. The impact of the line-width enhancement factor and the carriers' lifetime of the SOA on signal degradation are presented in Figure 15(c) and Figure 15(d), respectively. We simulate a cascade of SOAs with different line-width enhancement factor from 3 to 6 and carrier lifetime from 100 ps to 400 ps (again with a $100 \mathrm{~Gb}$ / s QPSK signal). We observe the same slope for all the cases and apply a common $P_{\text {in }} \times N$ convergence rule to demonstrate identical nature of the penalties [69]. As the line-width enhancement factor variations are less influential for high-degree M-QAM formats as con- 
cluded in reference [72], SOA with shorter carrier lifetime should be chosen for complex modulation signals.

\subsection{Experimental evaluation}

In this section, we confront the models with experimental results obtained with conventional, transmission SOAs. For the direct detection experiment, the ring consists of a repetition of four times a pattern made of a transmission link followed by an OPS/OSS node [6]. Each transmission link is composed of $50 \mathrm{~km}$ of standard SMF followed by dispersion compensating fiber to provide full chromatic dispersion compensation, in between EDFAs; $2-\mu \mathrm{s}-$ long data packets with (2 $\left.2^{31}-1\right)$-long PRBS are used at $1549.3 \mathrm{~nm}$. Our burst-mode receiver was designed to operate at $10 \mathrm{Gbit} / \mathrm{s}$ including 7\% forward error correction (FEC) and protocol overhead. BER measurements are done using a standard error counter. Before entering the burst-mode receiver, the signal performance is artificially degraded with a variable optical attenuator or a noiseloading device. The concept of the experimental setup for the coherent experiment is depicted in Figure 16. We use a dual-polarization I/Q modulator for the coherent detection experiments. Four digital-to-analog converters were used to drive the modulator, generating PDM signals at 28 Gbaud with diverse modulation formats: QPSK, 8-, and 16-QAM. All signals are based on PRBS of length $2^{15}-1$ bits. After traversing a polarization scrambler, the light enters into a recirculating loop emulating the propagation through many optical nodes. In order to emulate such nodes, an optical packet blocker is built with two wavelength-selective switches working as wavelength (de)multiplexer and a commercial SOA as optical gate and amplifier with 11 $\mathrm{dB}$ optical gain. A variable optical attenuator is used to control the launching power into the loop containing $25 \mathrm{~km}$ of SMF, SOA, and EDFA. Finally, optical signals are detected using a coherent mixer and four pairs of balanced photodiodes connected to a $20-\mathrm{GHz}$ bandwidth oscilloscope working at $40 \mathrm{GS} / \mathrm{s}$. All measured waveforms were processed offline [73].

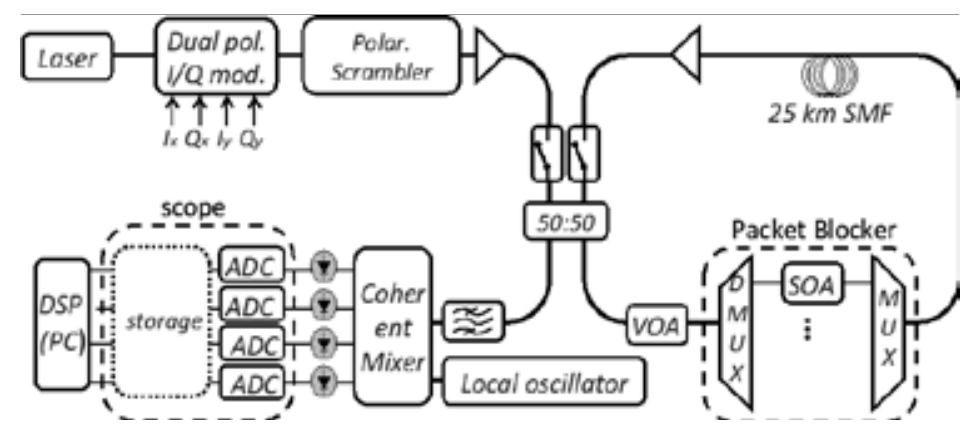

Figure 16. Experimental recirculation optical loop for high data rate coherent packet-switched network.

\subsubsection{NRZ-OOK optical packet experiments}

In the back-to-back experiment, the required OSNR yielding $10^{-3}$ BER was found at $11 \mathrm{~dB}$, corresponding to an extinction ratio of $r_{0}=13.5 \mathrm{~dB}$ from equation (16). We measured the OSNR penalties at $10^{-3}$ BER arising from a series of SOAs versus the IP. Up to four devices are 
cascaded. Their input optical power levels are varied over a large operation range, from -20 $\mathrm{dBm}$ to $7 \mathrm{dBm}$. We report the results in Figure 17(a). It can be observed that the plots gather almost on a single curve. This curve is found to match very well the predictions from the analytical model proposed in Section 3.3.1, when the data of Figure 8(a) are used as input parameters. Based on Figure 13(a) and Figure 17(a), we restrict the experimental measurements to only one SOA. The NLT can be easily derived, at $-3 \mathrm{dBm}$. Besides, we characterized NF of our transmission SOA, which was found to be $8 \mathrm{~dB}$. RSOAs share the same fundamental building block as SOAs; therefore, we will assume that all the above considerations also apply to RSOAs. Hence, we just need to characterize a single device in order to predict system performance in a multi-device network. We report the OSNR penalty versus integrated power [in Figure 17(b)] after one single module and derive the NLTs. We first inserted an RSOA device, optimized for access networks $\left(G=24 \mathrm{~dB}, P_{\text {sat }}=6.5 \mathrm{dBm}\right)$ [74]. The distortions due to nonlinearities were found prohibitively high for our applications. The measured NLT does not exceed $-17 \mathrm{dBm}$, that is, $14 \mathrm{~dB}$ worse than the transmission SOA. Then, we tested a specific RSOA with a reduced gain in order to contain nonlinearities $\left(G=7 \mathrm{~dB}, P_{\text {sat }}=4.1 \mathrm{dBm}\right)$. With these parameters, the NLT is increased to $-3 \mathrm{dBm}$, similar to the transmission $\mathrm{SOA}$, as reported in Figure 17(b). From a noise point of view, both RSOA devices exhibit a noise figure of $11 \mathrm{~dB}$, that is, $3 \mathrm{~dB}$ larger than for the transmission SOA. The analytical model of Section 3.3.1 has been proposed to predict the level of distortions versus optical power. Therefore, it can also be used to derive the optical power, which guarantees a given level of distortions (i.e., a given $\mathrm{BER})$. The evolution range of (R)SOA input powers with respect to the maximum number of cascaded nodes is presented in Figure 17(c) for SOA and RSOA devices. As described in Section 4.1.2, the maximum number of cascaded SOA could be found when the nonlinear and the ASE limits intersect. RSOAs specially designed for access network show high nonlinearity, which limits the maximum number of cascaded devices to five with $10 \mathrm{Gbit} / \mathrm{s}$ NRZ signal. With the RSOA of reduced optical gain, 26 devices can be cascaded. Optimized SOA with high saturation power allows even higher number of cascaded devices (up to 37).
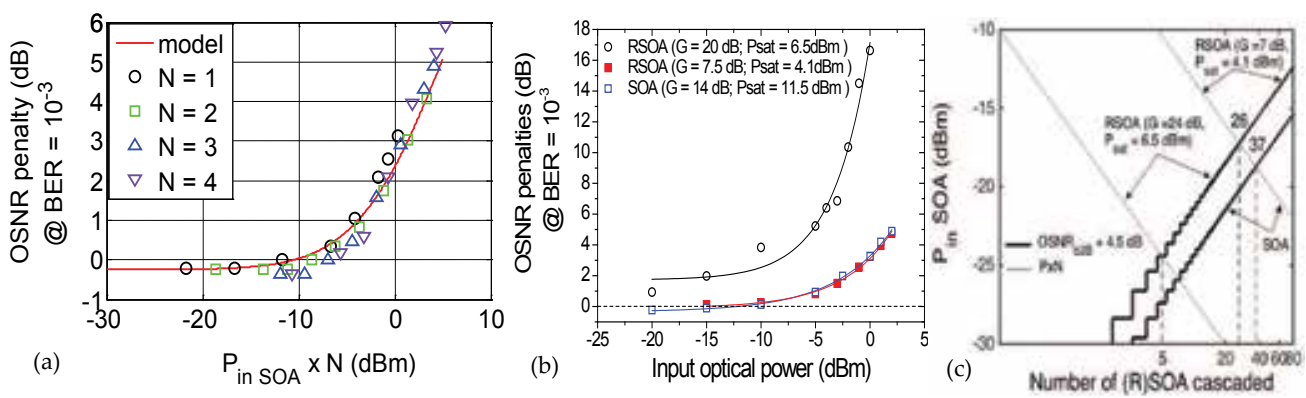

Figure 17. (a) Penalty for various configurations of SOA cascades. (b) Measured penalties caused by three types of (R)SOAs (one device per configuration, no cascade). (c) Number of cascades for SOAs and RSOAs devices for $10 \mathrm{Gbit} / \mathrm{s}$ OOK-NRZ signals. 


\subsubsection{PDM-QPSK, PDM-8QAM, and PDM-16QAM optical packets experiment}

In this section, the impairments produced in coherent signals when traversing a long cascade of SOAs are presented following the work done in reference [73]. The setup on Figure 16 allows for a full characterization of the performance in a network comprising unlimited number of SOAs. Moreover, the capability of controlling the input power into an SOA permits to determine the impact of traversing such device when working in different regimes. Finally, coherent detection enables to study the evolution of the whole optical field along the cascade of SOAs.

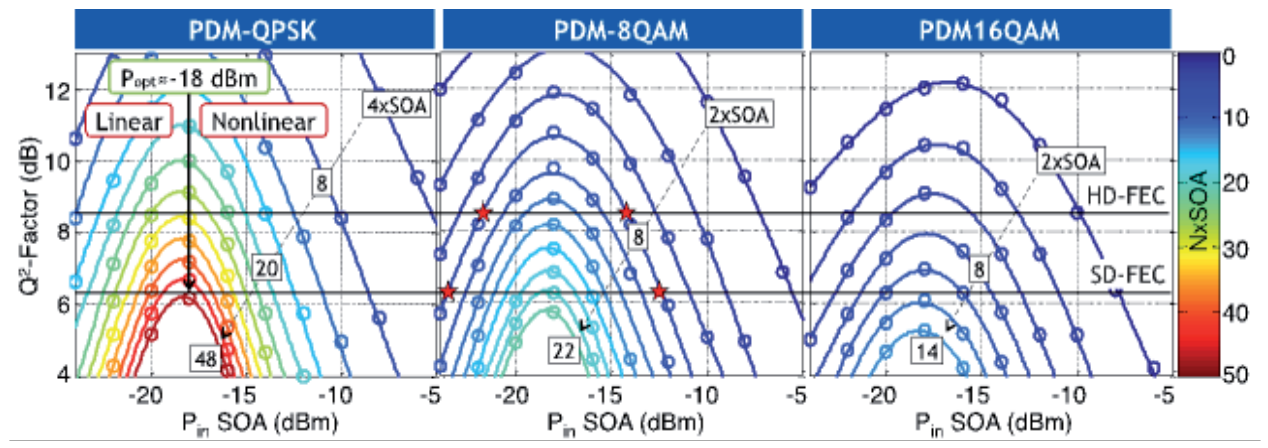

Figure 18. $\mathrm{Q}^{2}$-Factor versus input power in the SOA evaluated along the SOA cascade for different modulation formats.

Figure 18 depicts the performance of the different modulation formats when traversing the SOA cascade under different working regimes of such device. In such graphs, each curve represents the $\mathrm{Q}^{2}$-Factor as a function of the power inserted into the SOA $\left(\mathrm{P}_{\text {insOA }}\right)$ after traversing $N$ times through the device (see color scale and boxed numbering). Using a power range between -24 and $0 \mathrm{dBm}$, such results describe most realistic ranges of operation of the SOA. As can be seen, each curve shows a maximum performance when setting an optimal $P_{\text {insOA }} \approx-18 \mathrm{dBm}\left(P_{\text {opt }}\right)$ for most cases, corresponding to the $P_{\text {insOA }}$ at which the SOA gain starts saturating. Therefore, such input optical power distinguishes the linear regime $\left(P_{\text {inSOA }}<P_{\text {opt }}\right)$ from the nonlinear regime $\left(P_{\text {insOA }}>P_{\text {opt }}\right)$. As described in Section 4.1.1, the NLT can be extracted from the OSNR penalty depending on the IP. We compare the experimental data with the simulations proposed in Section 4.1.2. We use the 1.76-dB OSNR penalty to assess the NLT after the cascade of SOAs. We use a 28-Gbaud NRZ optical signal based on PRBS of length $2^{15}$ 1 bits as in the experiment. Concerning the SOA model, we use the provided parameters corresponding to our commercial SOA used in this experiment (optical gain of $11 \mathrm{~dB}$, saturation power of $13 \mathrm{dBm}$, and NF of $6.9 \mathrm{~dB}$ ). Then, we adjust the carrier lifetime as well as the alpha factor, which are not provided. We use a recombination carrier lifetime of $100 \mathrm{ps}$ and high alpha factor of $15 \mathrm{~dB}$ in order to increase the impact of the amplitude and phase deviations. The OSNR penalty versus the IP is presented in Figures 19(a) and (b) for PDM-8QAM and PDM-16QAM signals, respectively. Good matching is obtained, however, at the expense of the alpha factor. We believe such difference is due to the non-ideal signal generation/detection of our optical setup. 

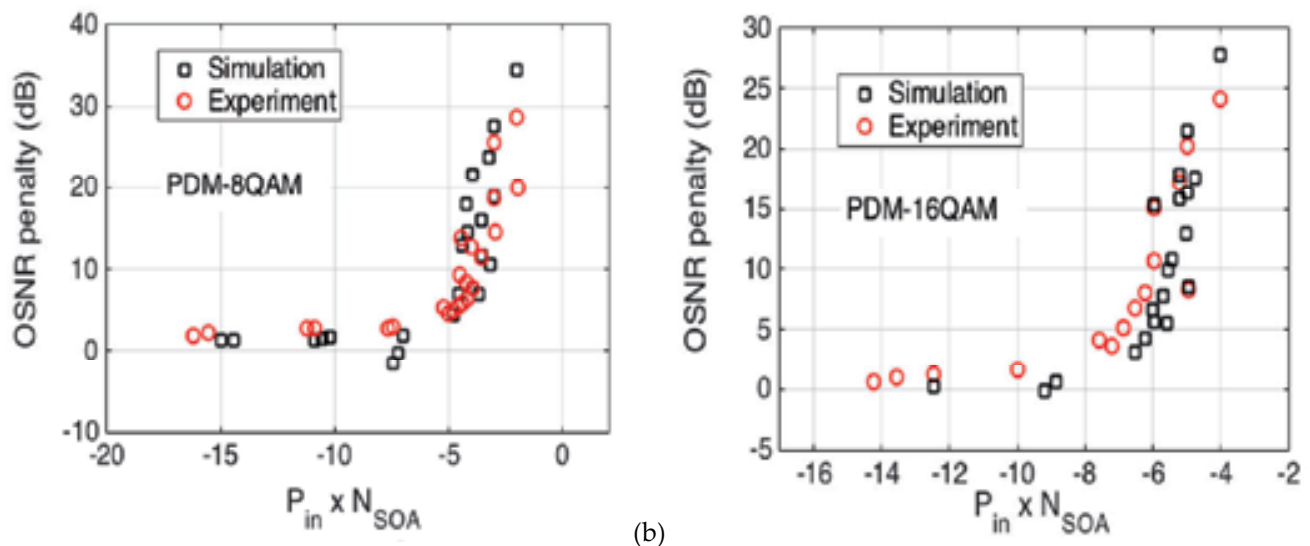

(a)

Figure 19. Modeled and experimental OSNR penalty depending on $N \times P_{\text {in SOA }}$ (a) for PDM-8QAM and (b) PDM-16QAM.

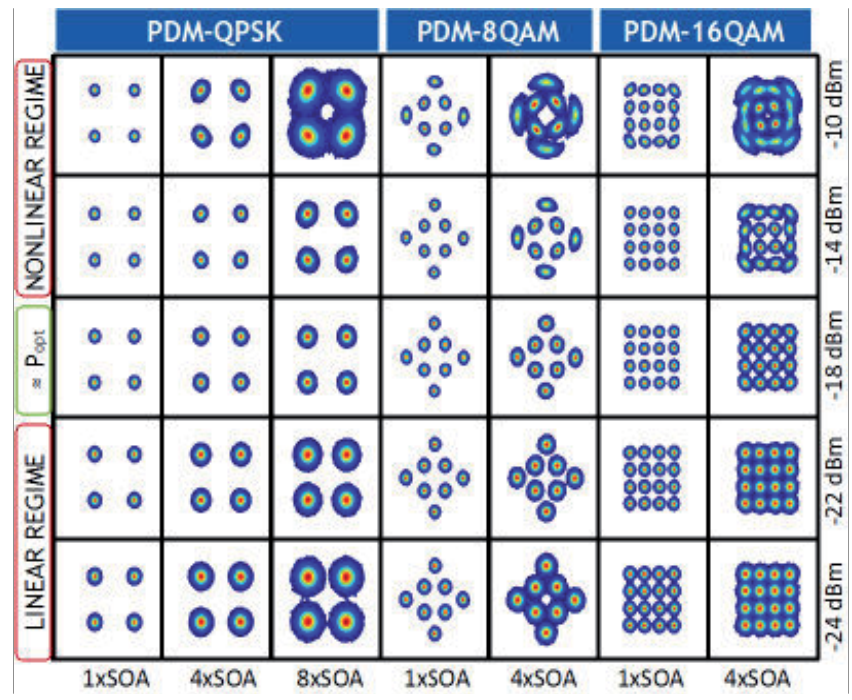

Figure 20. Exemplary constellations of recovered signals along the SOA cascade for different working regimes.

In Figure 20, we depict the recovered exemplary constellations after traversing several SOAs for different operating regimes. Irrespective of the modulation format, one can clearly notice that, in the linear regime, the constellations degrade isotropically along the cascade, which denotes the ASE noise being the major impairment. On the other hand, in the nonlinear regime, constellations evolve differently. In such regime, one can already observe by visual inspection that the phase noise grows faster than the amplitude noise, indicating the predominance of self-phase modulation as main distortion. One can notice that such impairment is even more critical for QAM signaling. This is due to greater amplitude oscillations of such modulation 
formats (due to the modulation format itself) with respect to QPSK, which amplitude only changes during inter-symbol transitions. It is important to remember that amplitude modulation induces a change of the SOA gain (when being under saturation), leading to SPM.

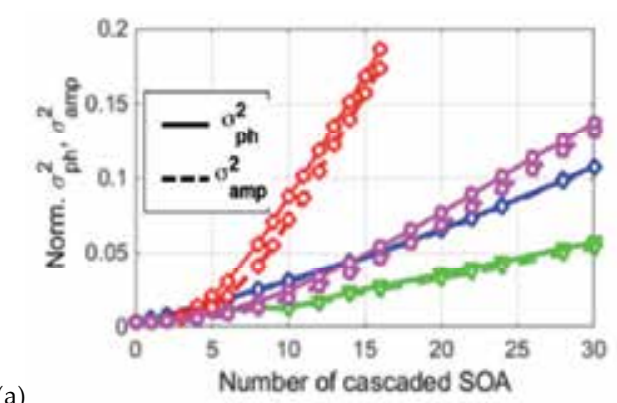

(b)

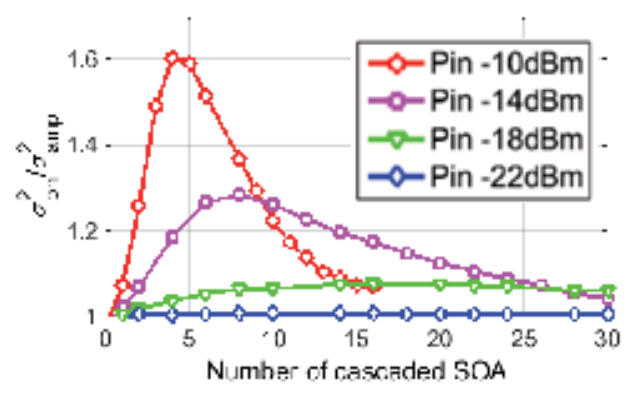

Figure 21. (a) Normalized variances and (b) phase-amplitude variance ratio evolution along the SOA cascade.

In order to study the noise evolution along the cascade, the phase and amplitude variances $\left(\sigma_{\mathrm{ph}}{ }^{2}\right.$ and $\sigma_{\mathrm{amp}}{ }^{2}$, respectively) for several $P_{\text {inSOA }}$ are calculated for the QPSK signal [see Figure $21(\mathrm{a})]$. When the input optical power is optimum $\left(P_{\text {insOA }}=-18 \mathrm{dBm}\right.$ as shown in Figure 18), the slope of the variances $\left(\sigma_{\mathrm{ph}}{ }^{2}\right.$ and $\left.\sigma_{\mathrm{amp}}{ }^{2}\right)$ as a function of the number of cascaded SOAs is minimum. Moving away from such optimum power leads to an increase of the slope, thereby leading to a faster degradation of the performances (as seen in Figure 18). In Figure 21(b), the ratio $\sigma_{\mathrm{ph}}{ }^{2} / \sigma_{\mathrm{amp}}{ }^{2}$ is plotted for the same conditions as in Figure 21(a). One can denote the increase of the phase with respect to the amplitude variance at high-input optical power. However, on the one hand, along the cascade, the constellation becomes more isotropic, meaning that $\sigma_{\mathrm{amp}}^{2}$ tends to approach $\sigma_{\mathrm{ph}}{ }^{2}$ (see QPSK constellations at $P_{\mathrm{inSOA}}=-10 \mathrm{dBm}$ in Figure 20 for visual aid). On the other hand, in the linear regime, all constellations remain mainly isotropic (see $P_{\text {insoA }}$ $=-22 \mathrm{dBm}$ in Figure 20).

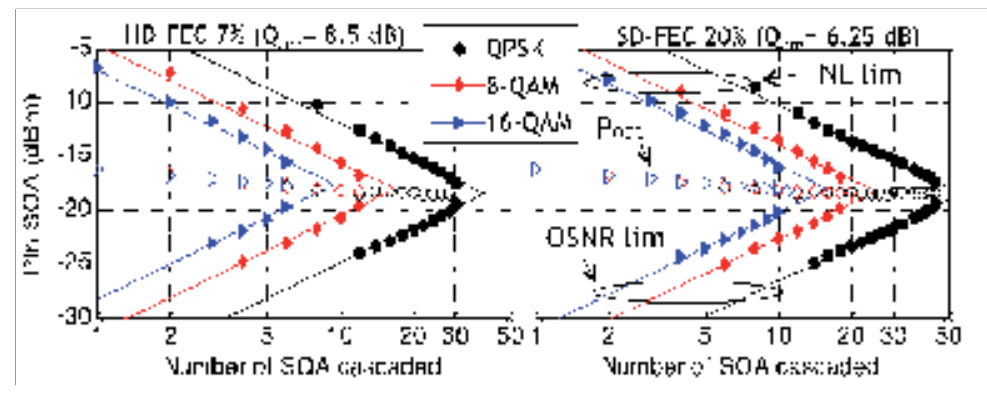

Figure 22. Experimental values (markers) and linear fit (lines) of nonlinear and OSNR limits that meet HD- and SDFEC requirements, and optimal power as a function of the number of cascaded SOAs.

The degradation observed due to the accumulation of distortions induced by the SOAs will limit the reach of the transmitted signals. Such reach depends on the forward error correction (FEC) scheme used to recover the signals. Typically, two FEC schemes might be applied: hard- 
decision (HD-) FEC with 7\% overhead or soft-decision (SD-) FEC with 20\% overhead, which requires $\mathrm{Q}^{2}$-factors of 8.5 and $6.25 \mathrm{~dB}$, respectively, to properly recover the information.

The nonlinear limit as well as the OSNR limit could be derived from the OSNR penalty versus the IP as presented in Figures 19(a) and (b). In this experiment, we directly look at the $\mathrm{Q}^{2}$-factor when moving below the considered FEC limits $(8.5$ and $6.25 \mathrm{~dB})$ at a given input SOA power and extract the maximum number of cascaded SOA (see stars in Figure 18 as example). As described in Section 4.2.1, the largest count of cascadable SOAs is obtained at the optimal tradeoff between distortions and noise, when nonlinear and OSNR limits cross. The results are presented in Figure 22 for both FECs. The empty markers show the evolution of $P_{\text {opt }}$ in order to guarantee the maximum number of cascaded devices. It increases from $-16 \mathrm{dBm}$ to around $-18 \mathrm{dBm}$ for all formats. One can observe that a large reach of up to 44,20 , and 11 nodes (1100, 500 , and $275 \mathrm{~km}$ ) can be achieved with QPSK, 8-, and 16-QAM signals, respectively, using SDFEC (30, 12, and 6 SOAs using HD-FEC). More details of this experiment are presented in reference [73]. One can observe that all fits (straight lines) match the experimental results, leading to a simple linear law that identifies the possible operational SOA range assuring performance for different modulations formats and FEC schemes. Design rules for SOA-based network could be derived as described by the first-order linear IP law (Section 4.1.1); however, such linear behavior is obtained in some specific scenario and an extra coefficient is to be added (general IP law) if different fiber length is considered, for example (as described by simulations in Section 4.1.2). Therefore, controlling the optical power variation between optical packets is crucial in order to operate the network in optimum conditions. Several techniques have been proposed in order to tackle this issue, such as employing an all-optical power equalization scheme based on two SOAs [75], hybrid optoelectronics control configuration [76,77] and dynamic gain control of SOAs [78,79]. We experimentally assess the system advantages of distributed power equalization scheme based on an automatic gain control SOA in a WDM packet-switched ring network composed of five cascaded network sections and show that error floor could be removed. More details can be found in reference [80].

\section{Conclusion}

Optical packet switching is a promising approach for highly efficient and dynamic optical networks allowing sub-wavelength networking directly at the optical layer. Furthermore, the reductions of optoelectronic conversion translate into energy savings. First, we reviewed the advantages of optical packet-switched network against circuit-switched, electronic packet, and optical transport networks. Then, we focused on the slot blocker implementation. This building block is a key enabler for efficient wavelength utilization. One potential candidate to fulfill the logic gate functionality is the SOA, due to its large optical bandwidth, low switching time, and high optical gain. In this chapter, we focused on the impact of SOA in a large cascade of OSS nodes. The SOA theory was discussed and we underlined several physical mechanisms that are responsible for the signal degradation. Thus, the SOA limits the signal reach due to the OSNR degradation by ASE and nonlinear distortions (mainly extinction ratio degradation and self-phase modulation) in the passing signal. We proposed an analytical model enabling to characterize the accumulation of SOA-based nonlinearities over an OOK-NRZ signal and 
experimentally assessed its accuracy. Different types of (R)SOA devices are studied and a convergence rule (IP law: $P_{\text {in }} \times N$ ) is proposed paving the way to new design rules for SOAbased OSS network (up to 37 SOA-based nodes could be cascaded). Then, by the means of numerical simulations, we assessed the impact of the cascade of SOAs on higher modulation formats such as QPSK, 8-QAM, and 16-QAM. We studied the role of SOA parameters in the amplitude and phase integrity of the optical signal. We verified the applicability of the IP law to the accumulation of nonlinearities in SOA cascade with QPSK and M-QAM signals and proposed modified IP rule $P_{\text {in }} \times N^{\gamma}$ for spans including fibers and SOAs. Finally, we experimentally studied a large cascade of SOA with several high-order modulation formats (PDMQPSK, 8-, and 16-QAM signals at 28 GBaud). Design rules could be extracted in order to guarantee error-free operation after different FECs. Long reach of 44, 20, and 11 SOA-based nodes has been achieved by PDM-QPSK, -8QAM, and -16QAM signals at 28 GBaud, respectively, thus establishing SOA as a promising candidate for coherent optical slot-switched networks.

\section{Author details}

Guilhem de Valicourt ${ }^{1 *}$, Miquel Angel Mestre Adrover ${ }^{2}$, Nikolay D. Moroz ${ }^{3}$ and Yvan Pointurier ${ }^{2}$

*Address all correspondence to: devalicourt@bell-labs.com

1 Bell Labs, Alcatel-Lucent, Holmdel, New Jersey, USA

2 Alcatel-Lucent Bell Labs France, Nozay, France

3 T8 Company, Moscow, Russia

\section{References}

[1] P. Winzer, "Beyond 100G Ethernet," IEEE Communications Magazine, vol. 48, no. 7, pp. 26-30, July 2010.

[2] G. Raybon, et al., "High Symbol Rate Transmission Systems for Data Rates above 400 Gb/s Using ETDM Transmitters and Receivers," in Proc. ECOC'14, Tu.3.3.5, Cannes, France, 2014.

[3] R. Rios-Müller, et al., "1-Terabit/s Net Data-Rate Transceiver Based on Single-Carrier Nyquist-Shaped 124 GBaud PDM-32QAM," in Proc. OFC'15, Th5B.1, Los Angeles, USA, 2015.

[4] Alcatel-Lucent Bell Labs, "Metro network traffic growth: an architecture impact study," Strategic white paper, 2013. 
[5] Y. Pointurier, et al., "Dimensioning and Energy Efficiency of Optical Metro Rings," in Proc. ONDM 2012, Colchester, 2012.

[6] D. Chiaroni, et al., "Packet OADMs for the next generation of ring networks," Bell Labs Technical Journal, vol. 14, no. 4, pp. 265-283, Winter 2010.

[7] H.-W. Chen, et al., "Forty Gb/s hybrid silicon Mach-Zehnder modulator with low chirp," Optics Express, vol. 19, no. 2, pp. 1455-1460, 2011.

[8] W. Zhang, et al., "Broadband silicon photonic packet-switching node for large-scale computing systems," IEEE Photonics Technology Letters, vol. 24, no. 8, pp. 688-690, 2012.

[9] H. Nishi, et al., "Monolithic Integration of a Silica-based Arrayed Waveguide Grating Filter and Silicon Variable Optical Attenuators based on p-i-n Carrier-Injection Structures," in Proc. ECOC 2010, We.8.E.3, Torino, 2010.

[10] G. de Valicourt, et al., "Monolithic Integrated Silicon-based Slot-Blocker for PacketSwitched Networks," in Proc. ECOC'14, We.3.5.5, Cannes, France, 2014.

[11] G. de Valicourt, et al., "Monolithic Integrated Reflective Polarization Diversity SOIbased Slot-Blocker for Fast Reconfigurable $128 \mathrm{~Gb} / \mathrm{s}$ and $256 \mathrm{~Gb} / \mathrm{s}$ Optical Networks," in Proc. ECOC'15, Tu.3.5.4, Valencia, Spain, 2015.

[12] A. Garreau, et al., "10 Gbit/s Drop and Continue Colorless Operation of a $1.5 \mu \mathrm{m}$ AlGaInAs Reflective Amplified Electroabsorption Modulator," in Proc. ECOC'06, We. 1.6.5, Cannes, France, Sep. 2006.

[13] G. de Valicourt, et al., "Reflective Packet Add-Drop Multiplexer based on Modulation Format Agnostic and Low Cost Optical Gate," in Proc. ECOC'12, We.2.B.5, Amsterdam, Netherlands, 2012.

[14] G. de Valicourt, et al., "A next-generation optical packet-switching node based on hybrid III-V/silicon optical gates," IEEE Photonics Technology Letters, vol. 26, no. 7, pp. 678-681, April 1, 2014.

[15] Y. Pointurier, et al., "Dimensioning and energy efficiency of multi-rate metro networks," IEEE/OSA Journal of Lightwave Technology, vol. 30, no. 22, pp. 3552-3564, 15 November 2012.

[16] Intune Networks, http://www.intunenetworks.com/.

[17] L. Dittmann, et al., “The European IST project DAVID: a viable approach toward optical packet switching," IEEE Journal of Selected Areas in Communication, vol. 21, no. 7, pp. 1026-1040, September 2003.

[18] M.C. Yuang, et al., "HOPSMAN: an experimental testbed system for a 10-Gb/s optical packet-switched WDM metro ring network," IEEE Communications Magazine, vol. 46, no. 7, pp. 158-166, July 2008. 
[19] J. Dunne, et al., “Optical Packet Switch and Transport: A New Metro Platform to Reduce Costs and Power by $50 \%$ to $75 \%$ While Simultaneously Increasing Deterministic Performance Levels," in Proc. BROADNETS, Madrid, Spain, September 2009.

[20] N. Deng, et al., "A Novel Optical Burst Ring Network with Optical-Layer Aggregation and Flexible Bandwidth Provisioning," in Proc. OFC, OThR5, Los Angeles, CA, March 2011.

[21] J.E. Simsarian, et al., "Fast-tuning coherent burst-mode receiver for metropolitan networks," IEEE Photonics Technology Letters, vol. 26, no. 8, pp. 813-816, April 2014.

[22] I. Widjaja, et al., "Light core and intelligent edge for a flexible, thin-layered, and costeffective optical transport network," IEEE Communications Magazine, vol. 41, no. 5, pp. S30-S36, May 2003.

[23] R. Luijten, et al., "Optical Interconnection Networks: The OSMOSIS Project," in Proc IEEE LEOS 2004, Rio Grande, Puerto Rico, November 2004.

[24] A. Stavdas, et al., "Dynamic CANON: a scalable multidomain core network," IEEE Communications Magazine, vol. 46, no. 6, pp. 138-144, June 2008.

[25] K.-I. Kitayama, et al., "Torus-topology Data Centre Network based on optical packet/ agile circuit switching with intelligent flow management," IEEE/OSA Journal of Lightwave Technology, vol. 29, no. 5, pp. 1063-1071, 1 March 2015.

[26] N.G. Basov, et al., "Production of negative-temperature states in p-n junctions of degenerate semiconductors," Soviet Physics, Journal of Experimental and Theoretical Physics, vol. 13, no. 6, pp. 1320-1321, 1961.

[27] M.G.A. Bernard and G. Duraffourg, "Laser conditions in semiconductors," Physica Status Solidi, vol. 1, no. 7, pp. 699-703, 1961.

[28] R.N. Hall, et al., "Coherent light emission from GaAs junctions," Physical Review Letters, vol. 9, no. 9, pp. 366-368, 1962.

[29] M.I. Nathan, et al., "Stimulated emission of radiation from GaAs p-n junctions," Applied Physics Letters, vol. 1, no. 3, pp. 62-64, 1962.

[30] H. Kroemer, "A proposed class of heterojunction injection lasers," Proceedings of the IEEE, vol. 51, no. 12, pp. 1782-1783, 1963.

[31] H. Kressel and H. Nelson, "Close-confinement gallium arsenide P-N junction lasers with reduced optical loss at room temperature," RCA Review, vol. 30, pp. 106-113, 1969.

[32] P.G. Kryukov and V.S. Letokhov, "Propagation of a light pulse in a resonantly amplifying (absorbing) medium," Soviet Physics Uspekhi, vol. 12, no. 5, pp. 641-672, March 1970. 
[33] L.W. Casperson, "Threshold characteristics of mirrorless lasers," Journal of Applied Physics, vol. 48, no. 1, pp. 256-262, January 1977.

[34] G. Zeidler and D. Schicetanz, "Use of laser amplifiers in glass fibre communication systems," Radio and Electronic Engineer, vol. 43, no. 11, pp. 675-682, 1973.

[35] S.D. Personick, "Applications for quantum amplifier in simple digital optical communication systems," Bell System Technical Journal, vol. 52, no. 1, pp. 117-133, 1973.

[36] J.C. Simon, "Polarisation characteristics of a travelling-wave-type semiconductor laser amplifier," Electronics Letters, vol. 18, no. 11, pp. 438-439, May 1982.

[37] T. Saitoh and T. Mukai, "A Low Noise $1.5 \mu \mathrm{m}$ GaInAsP Traveling-Wave Optical Amplifier with High Saturation Output Power," in ISLC, PD-5, Kanazawa, Japan, 1986.

[38] Y. Yamamoto, "Charasteristics of AlGaAs Fabry-Perot cavity type laser amplifiers," Journal of Quantum Electronics, vol. 16, no. 10, pp. 1047-1052, 1980.

[39] J.C. Simon, “GaInAsP semiconductor laser amplifiers for single-mode fibre communications," Journal of Lightwave Technology, vol. LT-5, no. 9, pp. 1286-1295, 1987.

[40] N.A. Olsson, et al., "Polarization-independent optical amplifier with buried facets," Electronics Letters, vol. 25, no. 16, pp. 1048-1049, 1989.

[41] D.M. Atkin and M.J. Adams, "Optical switching in the twin-guide travelling-wave laser amplifier," IEE Proceedings Journal, vol. 140, no. 5, pp. 296-300, October 1993.

[42] T. Kirihara, et al., "Lossless and low-crosstalk characteristics in an InP-based 4x4 optical switch with integrated single-stage optical amplifiers," Photonics Technology Letters, vol. 6, no. 2, pp. 218-221, February 1994.

[43] I. Valiente, et al., "Theoretical analysis of semiconductor optical amplifier wavelength shifter," Electronics Letters, vol. 29, no. 5, pp. 502-503, March 1993.

[44] T. Durhuus, et al., "All-optical wavelength conversion by semiconductor optical amplifiers," Journal of Lightwave Technology, vol. 14, no. 6, pp. 942-954, June 1996.

[45] L. Gillner, "Modulation properties of a near travelling-wave semiconductor laser amplifier," IEE Proceedings Journal, vol. 139, no. 5, pp. 331-338, October 1992.

[46] A.C. Labrujere, et al., "Phase modulation and optical switching by semiconductor laser amplifier," , ThC4, Technical Digest Series Optical Society of America, Washington, pp. 138-141, 1991.

[47] R. Schnabel, et al., "All Optical AND Gate Using Femtosecond Non-Linear Gain Dynamics in Semiconductor Optical Amplifiers," in Proc. ECOC'93, Montreux, Switzerland, pp. 133-136, September 1993.

[48] C. Joergensen, et al., "4 Gb/s Optical wavelength conversion using semiconductor optical amplifiers," Photonics Technology Letters, vol. 5, no. 6, pp. 657-660, June 1993. 
[49] S. Kawanishi, et al., "Ultrahigh-speed phaselocked-loop-type clock recovery circuit using a travelling-wave laser diode amplifier as a $50 \mathrm{GHz}$ phase detector," Electronics Letters, vol. 29, no. 19, pp. 1714-1716, September 1993.

[50] J. Leuthold, et al., "An All-Optical Grooming Switch with Regenerative Capabilities," in Proc. ICTON, Azores, Portugal, pp. 1-4, 2009.

[51] A.H. Gnauck, et al., "10 -Gb/s 360-km transmission over dispersive fiber using midsystem spectral inversion," Photonics Technology Letters, vol. 5, no. 6, pp. 663-666, June 1993.

[52] P.Y. Cortès, et al., "Below 0.3 dB Polarisation Penalty in 10 Gbit/s Directly Modulated DFB Signal Over $160 \mathrm{Km}$ using Mid-Span Spectral Inversion in a Semiconductor Optical Amplifier," in proc. ECOC'95, Brussels, Belgium, pp. 267-270, September 1995.

[53] L. Noel, et al., "Four WDM channel NRZ to RZ format conversion using a single semiconductor laser amplifier," Electronics Letters, vol. 31, no. 4, pp. 277-278, February 1995.

[54] G.P. Agrawal and A.N. Olsson. Self-phase modulation and spectral broadening of optical pulses in semiconductor laser amplifiers. IEEE Journal of Quantum Electronics. vol. 25, no. 11, pp. 2297-2306, 1989. DOI: 10.1109/3.42059.

[55] G.P. Agrawal, "Fiber Optics Communication Systems," Wiley Interscience, New York, 1992.

[56] M. Shtaif, et al., "Noise spectra of semiconductor optical amplifiers: relation between semiclassical and quantum descriptions," Journal of Quantum Electronics, vol. 34, no. 5, pp. 869-878, 1998.

[57] R. Brenot, et al., "Experimental Study of the Impact of Optical Confinement on Saturation Effects in SOA," in Proc. OFC 2005, OME50, Anaheim, CA, USA, March 2005.

[58] G. de Valicourt, et al., "10Gbit/s Modulation of Reflective SOA without Any Electronic Processing," in Proc. OFC'11, OThT2, Los Angeles, USA, 2011.

[59] G. de Valicourt, Chapter 1: "Next generation of Optical Access Network based on Reflective-SOA," Book: "Selected Topics on Optical Amplifiers in Present Scenario," Dr. Sisir Garai (Ed.), ISBN: 978-953-51-0391-2, InTech, (invited), 2012.

[60] C.H. Henry, "Theory of the linewidth of semiconductor lasers," IEEE Journal of Quantum Electronics, vol. 18, no. 2, pp. 259-264, 1982. DOI: 10.1109/JQE. 1982.1071522.

[61] F. Koyama and K. Iga, "Frequency chirping in external modulators," Journal of Lightwave Technology, vol. 6, no. 1, pp. 87-93, January 1988.

[62] T. Watanabe, et al., "Transmission performance of chirp-controlled signal by using semiconductor optical amplifier," Journal of Lightwave Technology, vol. 18, no. 8, pp. 1069-1077, August 2000. 
[63] G. de Valicourt, et al., "Cascadability of optical packet-switching nodes based on (R)SOA devices," IEEE Photonics Technology Letters, vol. 25, no. 24, pp. 2389-2392, December 15, 2013.

[64] J.-C. Antona, et al., "Physical design and performance estimation of heterogeneous optical transmission systems," Comptes Rendus Physique, vol. 9, no. 9-10, pp. 963-984, November 2008.

[65] D. Cassioli, et al., "A time-domain computer simulator of the nonlinear response of semiconductor optical amplifiers," IEEE Journal of Quantum Electronics, vol. 36, no. 9, pp. 1072-1080, 2000.

[66] A. Ghazisaeidi, et al., "Bit patterning in SOAs: statistical characterization through multicanonical Monte Carlo simulations," IEEE Journal of Quantum Electronics, vol. 46, no. 4, pp. 570-578, 2010. DOI: 10.1109/JQE.2009.2029545.

[67] P. Borri, et al., "Spectral hole-burning and carrier-heating dynamics in InGaAs quantum-dot amplifiers," IEEE Journal of Selected Topics in Quantum Electronics, vol. 6, no. 3, pp. 544-551, 2000. DOI: 10.1109/2944.865110.

[68] A. Ghazisaeidi and L.A. Rusch, "On the efficiency of digital back-propagation for mitigating SOA-induced nonlinear impairments," Journal of Lightwave Technology, vol. 29, no. 21, pp. 3331-3339, 2011.

[69] N.D. Moroz, et al., "On accumulated signal degradation in a cascade of semiconductor optical amplifiers," Journal of Physics: Conference Series, 584 012008, 2015.

[70] E. Grellier and A. Bononi, "Quality parameter for coherent transmissions with Gaussian-distributed nonlinear noise," Optics Express, vol. 19, no. 13, pp. 12781-12788, June 2011.

[71] F. Vacondio, et al., "On nonlinear distortions of highly dispersive optical coherent systems," Optics Express, vol. 20, no. 2, pp. 1022-1032, January 2012.

[72] R. Bonk, et al., "Linear semiconductor optical amplifiers for amplification of advanced modulation formats," Optics Express, vol. 20, no. 9, pp. 9657- 9672, April 2012.

[73] M.A. Mestre, et al., "On the SOA Cascadability and Design Rules for Optical PacketSwitched Networks," Optical Fiber Communication Conference, OSA Technical Digest (online) (Optical Society of America, 2015), paper Th2A.2.

[74] G. de Valicourt, et al., "High gain $(30 \mathrm{~dB})$ and High saturation power $(11 \mathrm{dBm})$ RSOA devices as colourless ONU sources in long reach hybrid WDM/TDM -PON architecture," IEEE Photonics Technology Letters, vol. 22, no. 3, pp. 191-193, Feb. 2010.

[75] S. Pato, et al., "On Using All-Optical Burst-Mode power Equalization in Converged Metro-Access Networks," ICTON 2009, We.B2.1, 2009 
[76] H. Wessing, et al., "Combining Control Electronics with SOA to Equalize Packet-toPacket Power Variations for Optical 3R Regeneration in Optical Networks at 10 Gbit/s," OFC'2004, paper WD2, 2004.

[77] A.V. Tran, et al., "Optical Packet Power Equalization with Large Dynamic Range using Controlled Gain-Clamped SOA," OFC 2005, OME46, 2005.

[78] N. Cheng, et al., "Long Reach Passive Optical Networks with Adaptive Power Equalization using Semiconductor Optical amplifiers," OSA/ACP'2009, paper FS4, 2009.

[79] Y. Kai, et al., "MSA Compatible Size, Dual-channel Fast Automatic Level Controlled SOA substems fro Optical Packet and PON signals," in Proc. ECOC'2011, Mo.2.A.2, 2011.

[80] G. de Valicourt, et al., "Distributed fast optical packet power equalization for efficient WDM packet switched networks," in Proc. ECOC'12, Tu.3.A.4, Amsterdam, Netherlands, 2012. 


\title{
Chapter 4
}

\section{All-optical Semiconductor Optical Amplifiers Using Quantum Dots (Optical Pumping)}

\author{
Khalil Safari, Ali Rostami, Ghasem Rostami and Mahboubed Dolatyari
}

Additional information is available at the end of the chapter

http://dx.doi.org/10.5772/62107

\begin{abstract}
In the first portion of this chapter, a short review on all-optical processing is presented. Following the ideas of all-optical processing, a basic unit cell is introduced for the realization of these systems. To this end, an all-optical semiconductor optical amplifier based on quantum dots (QD-SOA) is presented and used as the basic unit cell. Then, a novel scheme for a high-speed all-optical half-adder based on quantum dot semiconductor optical amplifiers has been theoretically and extensively analyzed. We accelerate the gain recovery process in QD-SOA with a control pulse (CP) using the cross-gain modulation (XGM) effect in QD-SOA (based on a novel work reported by Rostami et al published in IEEE J. Quantum Electron in 2010). In this proposed scheme, a pair of input data streams simultaneously drives the switch to produce sum and carry. The proposed scheme is driven by the pair of input data streasms for one switch between which the Boolean XOR function is to be executed to produce a sum-bit. Then, one of the input data is utilized to drive the second switch and another is used as input data for it to produce a carry-bit. In the proposed structure, we need to use an optical attenuator to reduce the power level of the optical signal. Thee, data pulse is at least an order of magnitude stronger than the incoming pulse; thereforehowever, only the input pulse can alter QD-SOA's optical properties. Also, an all-optical cross-phase modulation (XPM) wavelength converter has been utilized to obtain an all-optical AND gate, which is logic CARRY. Logic SUM and CARRY are simultaneously realized in the proposed structure. The operation of the system is evaluated and demonstrated with a $\mathrm{Tb} / \mathrm{s}$ bit rate. The proposed structure is mathematically modeled by writing rate equations and then is numerically simulated with success. High-speed operation capabilities of the proposed all-optical half-adder structure are evaluated by numerical simulation.
\end{abstract}

Keywords: All-optical Half-Adder, QD-SOA, Optical Pumping, High-speed Processing 


\section{Introduction}

In the past few years, all-optical signal processing concepts and technologies have evolved remarkably mainly due to the discovery of semiconductor-based all-optical switches. Electronic devices such as switches and routers are not fast enough, whereas the speed of optical communication systems can reach up to $10 \mathrm{~Tb} / \mathrm{s}$ [1]. Therefore, in order to realize these capabilities in optical engineering, recently, there washas been a huge motivation for researchers in this area to concentrate on the implementation of the optical digital logic gates (e.g., AND, OR, NOT, and XOR) and optical logic modules (e.g., counters, adders, subtractors, and shift registers) [2]. In SOAs, nonlinear effects such as cross-gain modulation (XGM), crossphase modulation (XPM), four-wave mixing (FWM), and transient cross-phase modulation can all be exploited to demonstrate all-optical signal processing functions. Several schemes on all-optical processing/operations, including nonlinear materials, terahertz optical asymmetric demultiplexer (TOAD), cross gain modulation in a semiconductor optical amplifier, SOAMach-Zehnder interferometer (SOA-MZI), Pperiodically poled lithium niobate (PPLN) waveguide, and ultrafast nonlinear interferometer (UNI), were proposed [3]. Most of the proposed designs are based on two or more interferometric switches; a synchronization problem between different switches restricts their designs for practical implementation. QDSOA has a remarkable ultrafast response, larger unsaturated gain, and a much faster gain recovery after gain compression than bulk SOAs, small electron relaxation times, highsaturation output power, low-noise figure, and large-gain-bandwidth product at the same time. One of basic demands for high-speed operation is high-speed carrier dynamics. In QDSOA, there is a high-speed carrier transport, therefore high-bit-rate devices such as logic gates can be realized based on this device. It is clear that due to band offset between QDS and wetting layer (WL), capture time will be much shorter than this parameter in bulk and quantum well structures. This parameter is between femtoseconds (fs) and picoseconds (ps) in QDs and therefore this is the main reason for high-speed operation of QD-SOA-based devices and systems. The response time for gain saturation is $100 \mathrm{fs}^{-1} \mathrm{ps}$, which is sufficient for a gigabit to sub-terabit optical transmission system [4]. Technology of the quantum-dot SOAs is suitable owing to its remarkably ultrafast response, which, combined with its attractive characteristics, distinguishes them from conventional SOAs. Compared to other types of amplifiers, One of other properties of the QD-SOA is a large unsaturated gain versus other type of amplifiers, which thatleadss to higher power output. Therefore, in low-injection electric pump, there is sufficient optical gain and thus less power consumption. Also, in electric pump case, optical gain can be controlled by current density and in optical pumpcase using intensity of pump signal too. QD-SOA illustrates faster gain recovery time versus other types of optical amplifiers. Thus, QD-SOA can be used for high-speed optical signal processing without distortion [5]. The all-optical logic gates, optical wavelength conversion, and in this chapter, we develop a theoretical approach for compensation of the carrier relaxation time into excited state (ES). In our model, we have considered two energy levels in both conduction and valence bands. It will be shown that applying a $\mathrm{CP}$ with enough energy will highly accelerate the recovery process of QD-SOA and will lead to a high-bit-rate operation of QD-SOA-MZI structure in the presence of the $\mathrm{CP}$. In recent years, the optical logic based on several different schemes has 
been demonstrated and reported, which is based on the dual-semiconductor optical amplifier Mach-Zehnder interferometer [6,7,8], semiconductor laser amplifier (SLA) loop mirror [5-8], ultrafast nonlinear interferometers [9-12], and four-wave mixing process in SOA [13] and other alternatives. In the past reported research results, there are several publications for the realization of all-optical half adders such as terahertz optical asymmetric demultiplexers and ultrafast nonlinear interferometers [14, 15]. Based on these excellent properties of QD-SOA, interferometric effect such as the Sagnac phenomenon was realized using this optical device [16-20]. Also, using nonlinear effects such as cross-phase modulation and cross-gain modulation, all-optical XOR and AND gates were implemented by Ki et al and finally the half-adder was implemented [16]. Considering the nonlinear properties of SOA, an all-optical half-adder operating in $10 \mathrm{Gbit} / \mathrm{s}$ was reported in Ref. [17]. Another proposal based on four-wave mixing in SOA was illustrated in Ref. [18]. The Hhalf-adder/subtractor unit based on dark-bright solitons has been reported in Ref. [19]. All-optical half-adder using planar three-core nonlinear directional coupler was presented in Ref. [20]. For completing all-optical half-adder, monolithically integrated SOA-based -MZI switches were used to provide compact size, thermal stability, high-speed compatibility, low switching energy, relative stability, and optical integration compatibility [21-23]. Here, we present a theoretical model of an ultrafast alloptical half-adder based on the two QD-SOA-based MZI, where, in the first switch, the pair of input data streams execute the Boolean XOR function to produce a sum-bit. Besides, an alloptical XPM wavelength converter has been utilized to obtain an all-optical AND gate, which is logic CARRY. Logic SUM and CARRY are simultaneously realized [24-26]. Our proposed design requires less number of switches and other auxiliaries as compared with the other halfadder circuits using semiconductor optical amplifier-based devices. The all-optical half-adder has the potential to execute the addition in the optical domain up to $2 \mathrm{~Tb} / \mathrm{s}$. The configuration of the proposed all-optical half-adders using two symmetrical QD-SOA-based MZI switches is presented as follows: Logic SUM and CARRY are simultaneously implemented to realize the all-optical half-adder. The all-optical half-adder uses only two input signals. In electrical engineering, all arithmetic-related functions including addition, subtraction, multiplication, and division are realized by two half-adders and are a prerequisite for all-optical processors [27]. Boolean functions of logic SUM and logic CARRY exactly coincide with the XOR gate and the AND gate.

\section{Principles and design of proposed all-optical half-adders}

The proposed structures for half-adders (schematics and SOA-based structures) are shown in Figs. 1 and 2. In order to implement the proposed half-adder in an optical domain, the alloptical XOR and AND gates are reviewed at first. To implement the all-optical XOR gate, two SOAs with cross-gain modulation are used. To implement the all-optical AND gate, binary characteristics of the XPM wavelength converter are used too. Since the operations of XOR and AND logic gates depend on XGM and XPM phenomenon in semiconductors, the maximum speed of half-adder can be increased up to the limit of XGM and XPM. The speed of operation for all-optical half-adders is limited by pulse width and recovery time in SOAs. 


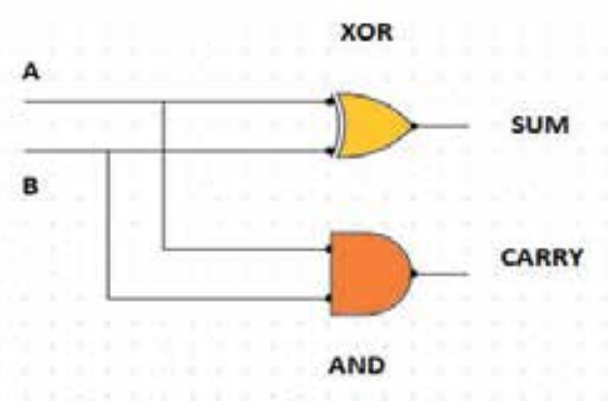

(a)

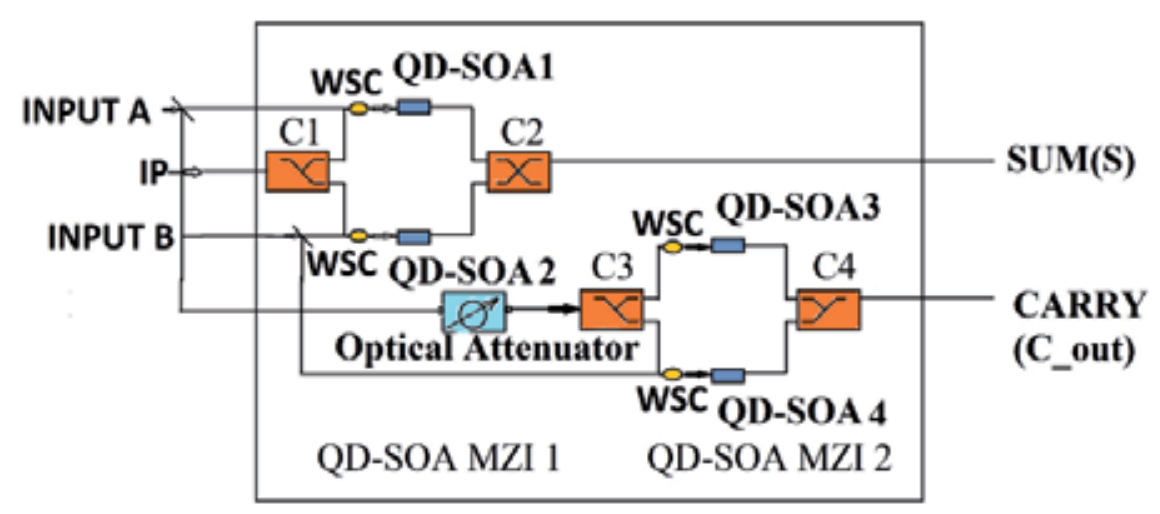

(b)

Figure 1. (a). Basic structure of half-adder [14]. (b). Half-adder without control pulse.

An electronic circuit (combinational circuits) performing addition of two binary digits is denoted as a half-adder. The carry bit will be 1 if both bits are 1, else will be zero. The sum-bit is the most significant bit of addition. A schematic of all-optical half-adders is illustrated in Fig. 2. It includes two MZIs in which QD-SOAs are in arms. For the first MZI, data inputs are $\mathrm{A}$ and B. Depending on data values A and B, the incoming pulse reaches Port- 1 or Port- 2 . In this structure, output Port- 1 corresponds to XOR operation and Port-2 corresponds to XNOR operation [3]. One of the attenuated input data acts as an incoming pulse for the second switch (MZI-2) and the other input data A (or B) produces the output of carry-bit. In other words, the output Port- 1 of the second switch gives the logic operation AND. The four possible cases are described as follows: the input data are 1 or zero when the light beam is present or absent, respectively. Based on Fig. 2, when $A=B=0$, the IP signal is applied only on the first MZI. Thus, we have the signal only from Port-2 and, Port-1 will be zero and Port- 2 will be 1 [3]. Therefore, Port- 1 becomes inactive. It generates the output as 0 , that is, carry $=0$. In the first switch (MZI- 1 ), if one of the input signals (either A or B) is 0 while the other is 1 , the incoming pulse emerges from the Port-1. Thuserefore, the sum (S) will be equal to 1 . Now, in the second switch (MZI-2), 


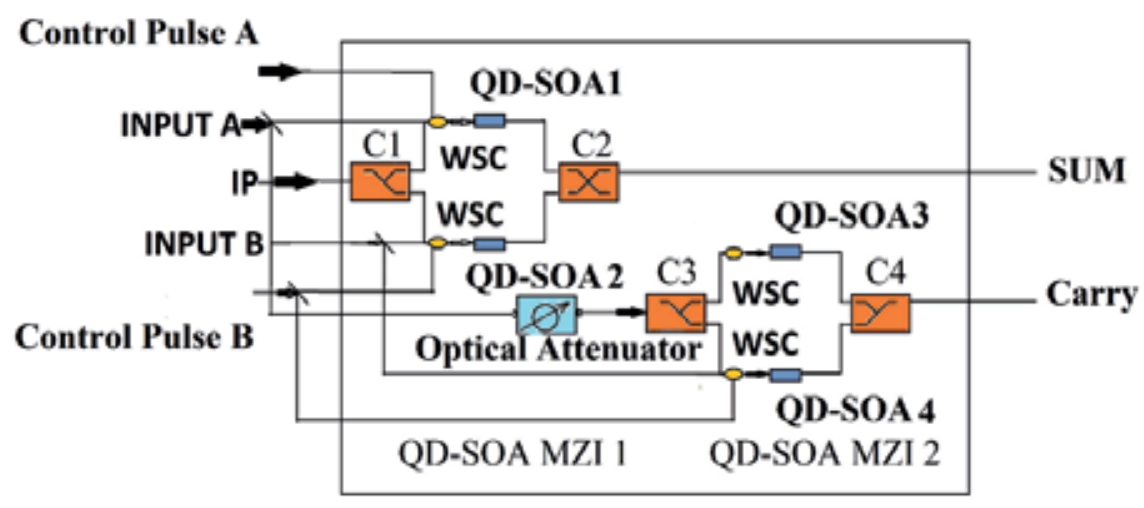

(a)

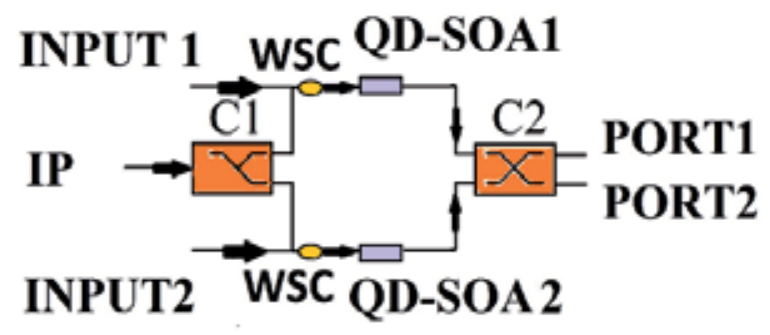

(b)

Figure 2. (a). Configuration of the proposed all-optical half-adder using two symmetrical QD-SOA-based MZI switches. (b). XOR gate [25].

AND gate produces the output as 0 , that is, carry $=0$. When the input signal is $1(A=B=1)$, the IP signal is applied on the first MZI as well. In this case, Port- 2 will be 1 . This way, the circuit performs the addition operation between two-bit binary data. In the next section, these theoretical results are verified through numerical simulations using Matlab [3].

\section{Operational principles of QD-SOA-based MZI switch}

The ease of manufacturing, installation, and operation of all-optical signal processing and communication needs integrated optics-based devices and systems [28]. As an example, the MZI is one of integrated optical building block and one can make SOA on. Thus, MZI-based SOA will be one of the integrated optical basic blocks [28]. Considering the basic principles of SOA-MZI for operation, logic gates show that it is well known when XPM and XGM or other nonlinear phenomenon in SOA is used while it is inserted in arms of MZI. In Fig. 3, a simple schematic of logic gates using SOA-MZI is shown, where SOA operates as a nonlinear element. In this structure, the pump light controls dynamics of nonlinear effects in SOA and then probe 
light propagates inside the nonlinear media and experiences nonlinear optical effects. On analyzing this structure, it is observed that the control and probe fields can return to zero or non-return to zero light. Based on carrier, depletion that is occurred in SOA will conclude to gain and phase modulation which named as XGM and XPM. It should be mentioned that these effects are so efficient and thus in SOA and QD-SOA it can be realized in very short length and low pump power. As we mentioned earlier, since optical pump light is amplified in SOA, therefore low pump power is required. As shown in Fig. 3, low-gain recovery time in SOAs can be compensated in the MZI- arrangement when SOAs are in both arms as differential form. In Fig. 4, total carrier variation in SOAs using symmetric- Mach--Zehnder (SMZ) gate-based gaiting window is illustrated. Using a control pulse with given pulse duration, a repetition rate is introduced to make a periodic variation in carrier density. When a short pulse width is used, the carrier density is depleted and also slow recovery time can be compensated by exciting both arms in MZI with suitable delay time. Based on the proposed ideas, the rise and fall times are defined by the control pulse duration [28].

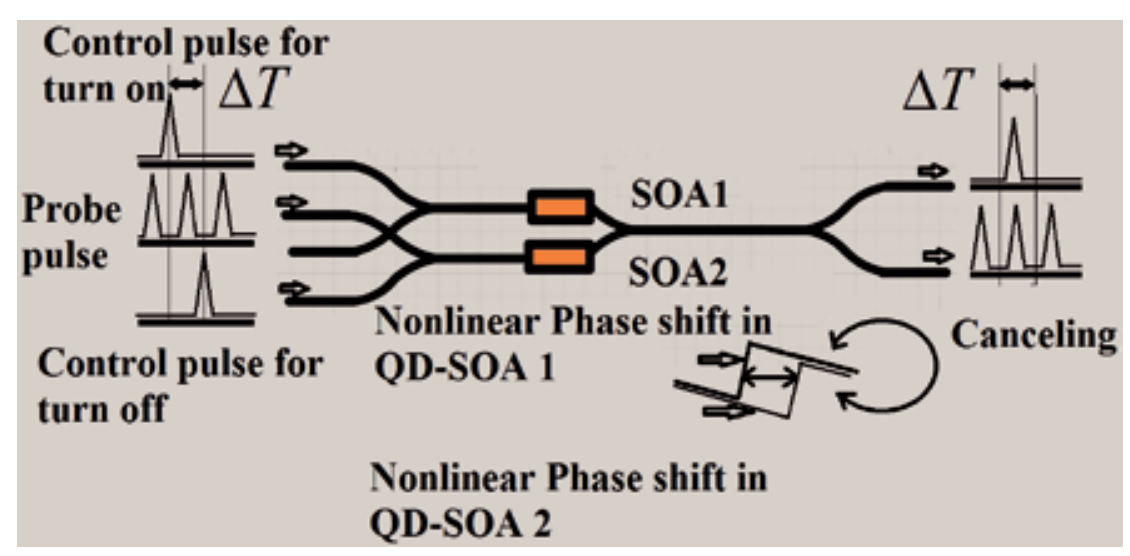

Figure 3. SMZ configuration and nonlinear phase response cancel out mechanism [26].

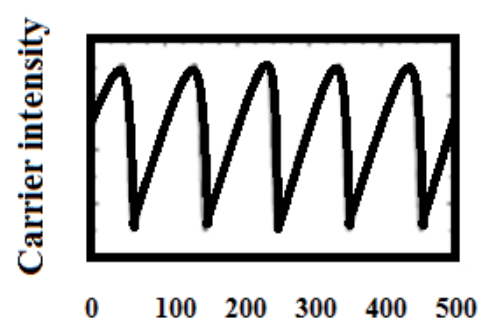

Time(ps)

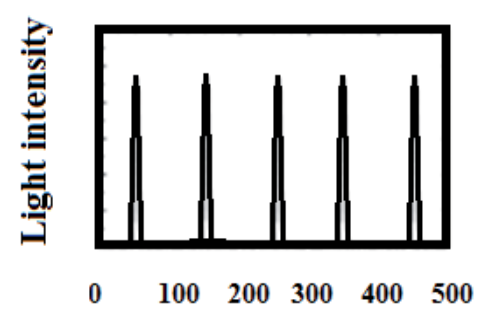

Time(ps)

Figure 4. Total carrier density of the SOAs in the presence of control pulses (left) and SMZ gate output (right) [26]. 


\subsection{SOA-MZI transfer function}

MZIs with SOAs on arms are the most suitable structures for applications such as optical logic gates. Considering similar applications such as other fiber-based devices, including SOAs, and nonlinear elements, such as semiconductor laser amplifier loop optical mirror (SLALOM) and terahertz optical asymmetric demultiplexer, the SOA in MZI structure presented in Fig. 5 can be modeled with a nonlinear device with a gain effect and a phase shift applied on the input signal [28]. Therefore, the transfer function of the considered structure can be derived in the the following manner. In the above configuration, $\left(A_{1}, A_{2}\right)$ and $\left(D_{1}, D_{2}\right)$ are input and output lightwaves, respectively, $\left(k_{1}, k_{2}\right)$ are normalized coupling coefficients of the input and output couplers and $\left(B_{1}, B_{2}\right)$ and $(C 1, C 2)$ are input and output lightwaves of the SOAs, respectively. The gain and phase shift of each of the SOAs are considered with $\left(G_{1}, \varphi_{1}\right)$ and $\left(G_{2}, \varphi_{2}\right)$ for upper and lower arms of SOAs. Thus, considering both pairs of input and output lightwaves, the following coupled equations can be obtained [28]:

$$
\left(\begin{array}{l}
B_{1} \\
B_{2}
\end{array}\right)=\left(\begin{array}{cc}
\cos K_{1} & i \sin K_{1} \\
i \sin K_{1} & \cos K_{1}
\end{array}\right)\left(\begin{array}{l}
A_{1} \\
A_{2}
\end{array}\right)
$$

We assumed that an optical signal travelling through the amplifier would experience an amplification of $\sqrt{G}$ gain and a phase shift of $\varphi$. Therefore,

$$
\left(\begin{array}{l}
C_{1} \\
C_{2}
\end{array}\right)=\left(\begin{array}{cc}
\sqrt{G_{1}} e^{i \Phi_{1}} & 0 \\
0 & \sqrt{G_{2}} e^{i \Phi_{2}}
\end{array}\right)\left(\begin{array}{l}
B_{1} \\
B_{2}
\end{array}\right)
$$

Then, the transfer function can be described as

$$
\left(\begin{array}{l}
D_{1} \\
D_{2}
\end{array}\right)=\left(\begin{array}{ll}
H_{11} & H_{12} \\
H_{21} & H_{22}
\end{array}\right)\left(\begin{array}{l}
A_{1} \\
A_{2}
\end{array}\right)
$$

where

$$
\begin{aligned}
& H_{11}=\cos K_{1} \cos K_{4} \sqrt{G_{1}} e^{i \Phi_{1}}-\sin K_{1} \sin K_{4} \sqrt{G_{2}} e^{i \Phi 2} \\
& H_{21}=i\left(\cos K_{1} \sin K_{4} \sqrt{G_{1}} e^{i \Phi_{1}}+\sin K_{1} \cos K_{4} \sqrt{G_{2}} e^{i \Phi 2}\right) \\
& H_{21}=i\left(\cos K_{1} \sin K_{4} \sqrt{G_{1}} e^{i \Phi_{1}}+\sin K_{1} \cos K_{4} \sqrt{G_{2}} e^{i \Phi 2}\right) \\
& H_{22}=-\sin K_{1} \sin K_{4} \sqrt{G_{1}} e^{i \Phi_{1}}+\cos K_{1} \cos K_{4} \sqrt{G_{2}} e^{i \Phi 2}
\end{aligned}
$$




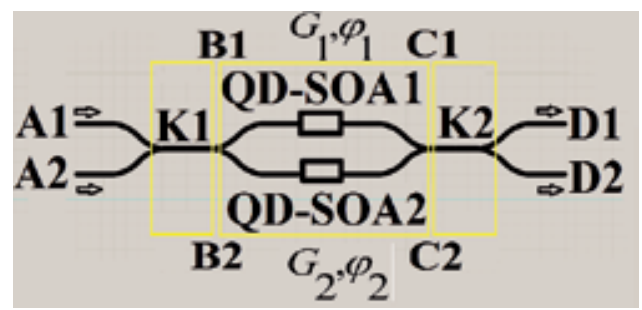

Figure 5. Schematic of SOA-incorporated MZI structure [26].

Denoting the input and output signal powers with PA1, PA2, PD1, and PD2 and assuming an ideal $3 \mathrm{~dB}$ coupler $\left(\sin k_{i}=\sqrt{2} / 2, \cos k_{i}=\sqrt{2} / 2\right)$, the transfer function reduces to

$$
\begin{aligned}
& H_{D 1}=\frac{P_{D 1}}{P_{A 1}}=\frac{1}{4} G_{1}+\frac{1}{4} G_{2}-\frac{1}{2} \sqrt{G_{1} G_{2}} \cos \Delta \Phi \\
& H_{D 2}=\frac{P_{D 2}}{P_{A 1}}=\frac{1}{4} G_{1}+\frac{1}{4} G_{2}+\frac{1}{2} \sqrt{G_{1} G_{2}} \cos \Delta \Phi \\
& \Delta \Phi=\Phi_{1}-\Phi_{2}
\end{aligned}
$$

\subsection{QD-SOA-MZI-based XOR gate}

Fig. 3 depicts a schematic diagram of the all-optical QD-SOA-based MZI switch. It consists of symmetrical MZI where one QD-SOA is located in each arm of the interferometer [3,1,23]. A probe signal composed of continuous series of unit pulses at wavelength $\lambda_{p}$ is inserted in the MZI and is split into two equal parts and travels separately along the identical QD-SOAs located in their path.

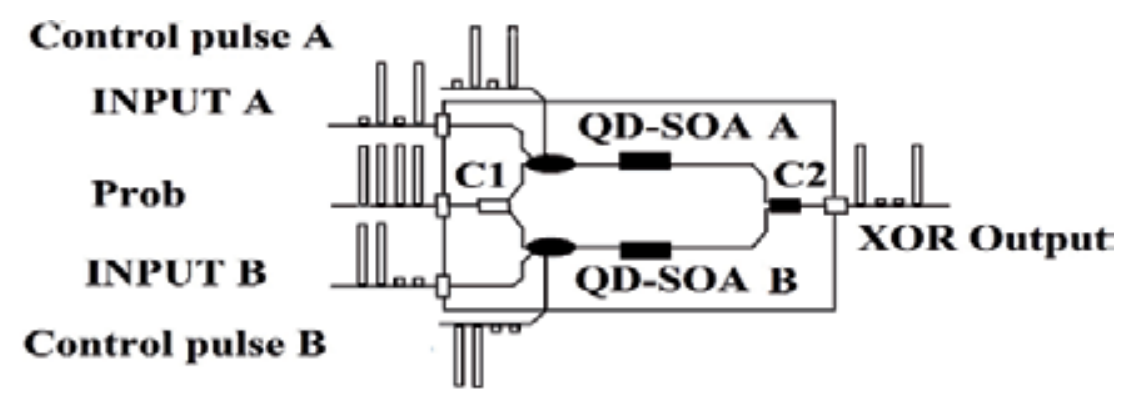

Figure 6. XOR gate based on QD-SOA [3].

The wavelength separation between $\lambda_{S}$ and $\lambda_{p}$ should be less than the homogeneous broadening of the single QD gain to ensure effective cross-gain modulation. The data pulse is at least 
an order of magnitude stronger than the incoming pulse so that only the input pulse can alter QD-SOAs' optical properties. In the case of input data $\mathrm{A}=\mathrm{B}=0$, the traveling probe signal through the two arms of the SOA acquires a phase difference of $\pi$ when it recombines at the output, and so the output is " 0 " due to the destructive interference. Besides, in the case of $A=1, B=0$, the signal traveling through the arm acquires a phase change due to the presence of XPM between the pulse train and the signal. The signal traveling through the lower arm does not have this additional phase change which results in an output " 1 ". The same result occurs for $A=0$, and $B=1$. When $A=1$ and $B=1$, the phase changes for the signal traveling through both arms will be equal, and the output is " 0 ". The XOR output intensity can be expressed as $[27-29,32]$

$$
P_{\text {XOR }}=P_{\text {probe }}\left\{k_{1} k_{2} G_{A}(t)+\left(1-k_{1}\right)\left(1-k_{2}\right) G_{B}(t)-2 \sqrt{k_{1} k_{2}\left(1-k_{1}\right)\left(1-k_{2}\right) G_{A}(t) G_{B}(t)}\right\} \times \cos \left[\varphi_{A}-\varphi_{B}\right]
$$

Where $G_{A}(t)$ and $G_{B}(t)$ are defined as integrated gain of QD-SOA, $\varphi_{1}(t), \varphi_{2}(t)$, are nonlinear phase shifts, $k_{1}, k_{2}$ are the coupling coefficients of the couplers $C_{1}$ and $C_{2}$, respectively, and, in this work, are equally set to 0.5 for simplicity. We present some recommendations for the parameter designs for practical QD-SOA devices.

1. The dynamics of gain recovery of QD-SOA is critically dependent on injection current density and electron relaxation time $\left(\tau_{w 2}\right)$ from WL to QD. Injecting higher current and decreasing $\tau_{w 2}$ provides faster gain recovery and consequently better gate performance. However, very high current is prohibited for practical devices.

2. Long-length QD-SOA provide high-quality factor, but high current is required to achieve the same current density.

3. Using multilayer QDs provide high density of QDs and relatively high-modal gain, but may reduce the saturation power and cause the pattern effect. Therefore, the number of QD layers should be appropriated according to the practical requirement.

4. Smaller $g_{0}$ produces narrower gate window width but lower window height.

5. Smaller $\alpha$ aids to produce narrower gate window. Low $\alpha$ value is the unique advantage of QD-SOA, so better gate performance can be expected.

6. Shorter pulse width assures smaller overlaps between pulses, and consequently highquality output signals.

7. Relatively high-control pulse energy can improve the quality factor $(Q)$ of the output signal [33-40].

\subsection{QD-SOA-MZI-based AND gate}

Logic AND operation is another important Boolean function which corresponds to the sampling of one signal with another. The AND gate is obtained using cross-phase modulation of two input signals in SOAs located in the two arms of a Mach-Zehnder interferometer built using SOAs. The principle of logic AND using the MZI involves coupling the two input signals 
into ports 1 and 3 of the SOA-MZI configuration. If the operation is performed on the positive slope in the transfer function of interferometer, the input signal 2 will sample input signal 1. Therefore, the selective switching of the data pulses at $\lambda_{\mathrm{AND}}$ occurs exclusively during the mark of signal 1, which yields the logical AND operation. The structural parameters are similar to the parameters considered for XOR gate [26, 41-44].

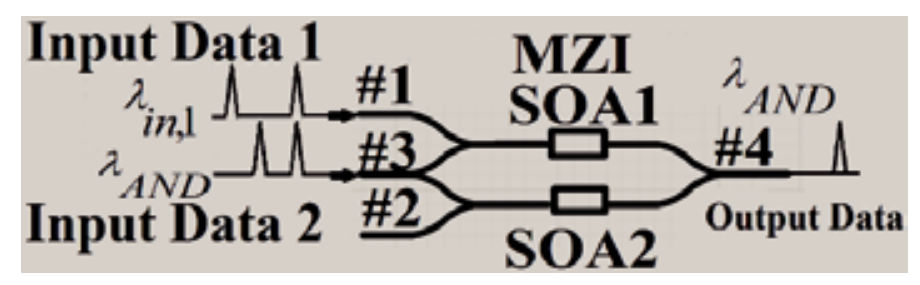

Figure 7. Configuration of a SOA-MZI structure for AND operation [26].

\subsection{Metrics characterizing the quality of switching in this chapter}

The quality factor $Q=20 \times \log \left(\left(P_{1}-P_{0}\right) /\left(\sigma_{1}+\sigma_{0}\right)\right)$ where $P_{1}, P_{0}$, and $\sigma_{1}$, and $\sigma_{0}$ are the mean and the standard deviation of the peak power of the output's ' 1 's and ' 0 's, respectively. $Q$ value is sensitive to the input pulse width and increasing the pulse width decreases the $Q$ factor because of the overlapping of two neighboring pulses. The quality factor $(Q)$ is dependent on $\Gamma$. Multilayer QD structures are considered as a technique to increase the modal gain due to increase in the $\Gamma$ parameter and therefore reducing the current threshold. The extinction ratio (ER) is defined as ER $=10 \log \left(\frac{P_{\mathrm{MIN}}^{1}}{P_{\mathrm{MAX}}^{0}}\right)$ where $P^{1}{ }_{\mathrm{MIN}}$ and $P^{0}{ }_{\mathrm{MAX}}$ are the minimum and maximum values of the peak power of high state and low state, respectively.

\section{Rate equation}

The typical structure of the quantum-dot SOA is illustrated in Fig. 5. The physics of operation of SOA includes the current injection into the active layer having quantum dots, and therefore the input optical signals are amplified through the stimulated emission or the use of the optical nonlinearity by the quantum dots for this processing. Fig. 5 also shows the cross-sectional and plan-view images of self-assembled InGaAs quantum dots as a typical example of quantumdot crystals. As it is clear, self-assembled InGaAs quantum dots on GaAs substrates and their application to semiconductor lasers have been studied since the early 1990s. They are nanosized semiconductor islands with a wetting layer grown via the Stranski-Krastanov mode under highly mismatched epitaxy, where the electron energy states are completely quantized due to the three-dimensional quantum confinement. Quantum-dot SOAs are novel optical devices using self-assembled quantum dots [34]. 


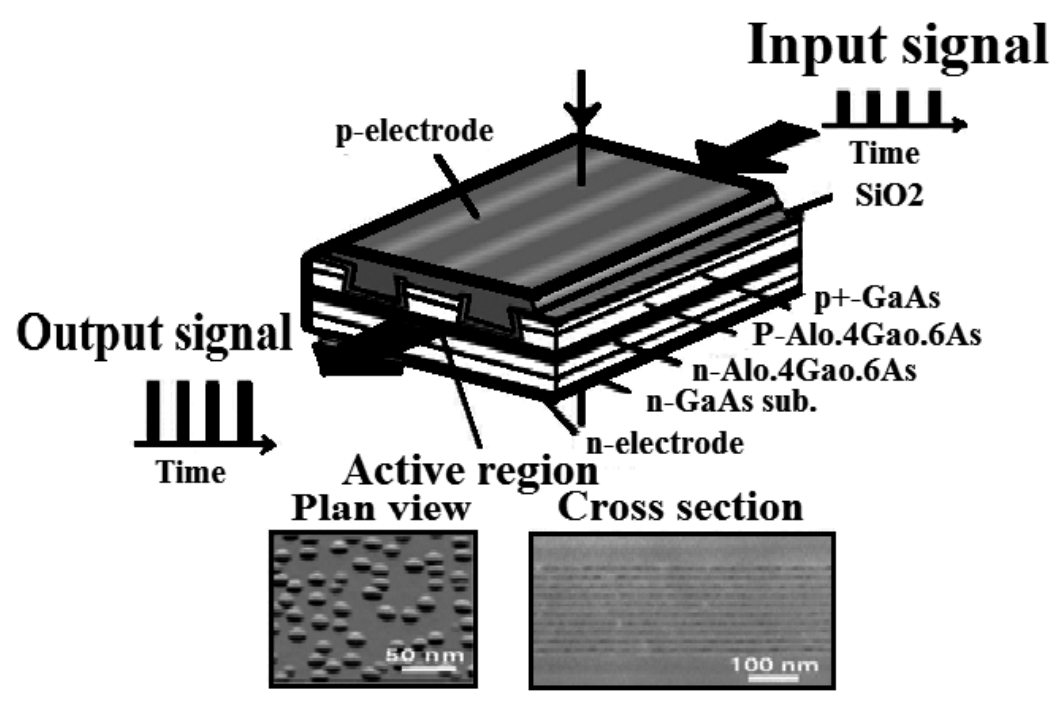

Figure 8. Structure of the quantum-dot SOA with cross-sectional and plan-view images of self-assembled InGaAs quantum dots. [34]

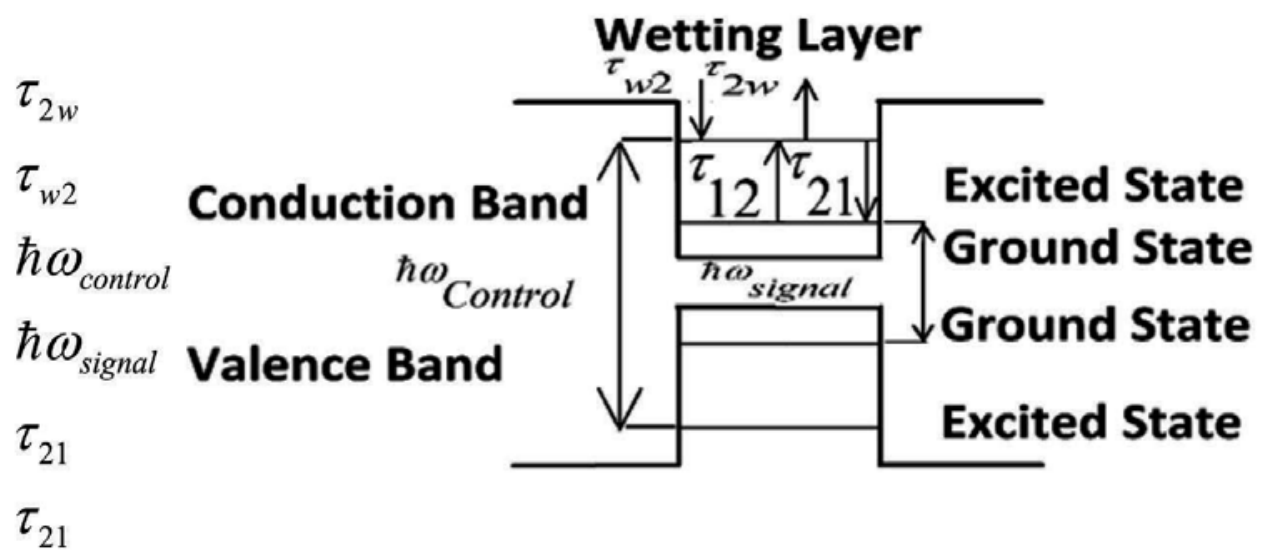

Figure 9. Band diagram of the QD structure with related energy levels [3].

In the QD-SOA-MZI, optical signals propagate in an active medium with the gain determined by the rate equations for the electron transitions in QD-SOA between WL, ground state (GS) and ES. We have considered the two energy levels in the conduction band: GS and ES. The diagram of the energy levels and electron transitions in the QD conduction band is shown in Fig. 5. The stimulated and spontaneous radiative transitions occur from GS to the QD valence band. The system of the rate equations accounts for the following transitions:

1. The fast electron transitions from WL to ES with the relaxation time $5 \times 10^{10} \mathrm{~cm}^{-2}$. 
2. The fast electron transitions between ES and GS with the relaxation time from ES to GS $\tau_{21}=0.16 \mathrm{ps}$ and the relaxation time from GS to ES $\tau_{21}=1.2 \mathrm{ps}$.

3. The slow transitions of electrons escaping from ES back to WL with the electron escape time $\tau_{2 w}=1 \mathrm{~ns}$.

The balance between the WL and ES is determined by the shorter time of QDs filling. Carriers relax quickly from the ES level to the GS level, while the former serves as a carrier reservoir for the latter. In general, the radiative relaxation time depends on the bias current. However, it can be shown that for moderate values of the WL carrier density $N_{w}=\left(10^{14}-10^{15}\right)$, this dependence can be neglected. The spontaneous radiative time in QDs $\tau_{1 R}$ remains large enough: $\tau_{1 R} \geq(0.4-0.5) n s$ [1]. In the case of the smaller signal detuning than the QD spectrum homogeneous broadening, the electron rate equations have the following forms $[3,1,35,36]$ :

$$
\begin{gathered}
\frac{\partial S_{\text {control }}(z, \tau)}{\partial z}=\left(-\alpha_{\text {abs }}\right) S_{\text {control }} \\
\frac{\partial S_{\text {signal,prob }}(z, \tau)}{\partial z}=\left(g\left(h w_{\text {signal,prob }}\right)-\alpha_{\text {int }}\right) S_{\text {signal,prob }} \\
\frac{\partial N_{w}(z, \tau)}{\partial \tau}=\frac{J_{0}}{e L_{w}}-\frac{N_{w}(1-h)}{\tau_{w 2}}+\frac{N_{w} h}{\tau_{2 w}}-\frac{N_{w}}{\tau_{\text {wr }}} \\
\frac{\partial f(z, \tau)}{\partial \tau}=\frac{(1-f) h}{\tau_{21}}-\frac{f(1-h)}{\tau_{12}}-\frac{f^{2}}{\tau_{1 R}}-\frac{g_{\text {max }, S} L}{N_{Q}}(2 f-1) \\
\times S_{\text {Prob }}(z, \tau) \frac{c}{\sqrt{\xi_{r}}}--\frac{g_{\text {max }, P} L}{N_{Q}}(2 f-1) \times S_{\text {signal }}(z, \tau) \frac{c}{\sqrt{\xi}} \\
\frac{\partial h(z, \tau)}{\partial \tau}=\frac{L_{w} N_{w}(1-h)}{N_{Q} \tau_{w 2}}-\frac{N_{w} L_{w} h}{N_{Q} \tau_{2 w}}-\frac{(1-f) h}{\tau_{21}}+\frac{f(1-h)}{\tau_{12}}+\frac{\alpha_{\max } L}{N_{Q}}(1-2 h) \times S_{\text {control }}(z, \tau) \frac{c}{\sqrt{\xi_{r}}}
\end{gathered}
$$

where $e$ is the electron charge and $J$ is the injection current density and bias current equal to $50 \mathrm{~mA}$. In addition, $\tau_{w 2}$ is the electron relaxation time from the WL to the $\mathrm{ES}, \tau_{2 w}$ is the electron escape time from the ES to the $\mathrm{WL}, \tau_{w R}$ is the spontaneous radiative lifetime in $\mathrm{WL}, \tau_{21}$ is the electron relaxation time from the ES to the GS, $\tau_{12}$ is the electron escape time from the GS to the ES, and $\tau_{1 R}$ is the spontaneous radiative lifetime in the QD. $N_{Q}$ is the surface density of QDs where its typical value is $5 \times 10^{10} \mathrm{~cm}^{-2} \alpha^{\prime} N_{w}$ is the electron density in the WL, $L{ }_{w}$ is the effective thickness of the active layer, $\xi_{r}$ is the SOA material permittivity and is the velocity of light in free space. The last term in eq. (5) and last two terms in eq. (6) demonstrate the 
absorption of $\mathrm{CP}$ and stimulated emission in the conduction band ground state (CBGS), respectively. For simplicity, we presume an ideal facet reflectivity and neglect the amplified spontaneous emission. The time dependence of the integral QD-SOA gain and pulse-phase shift can be expressed as $G(\tau)=\exp \left(\int_{0}^{L} g\left(z^{\prime}, \tau\right) d z\right)$ and $\phi(\tau)=-\alpha / 2\left(\int_{0}^{L} g\left(z^{\prime}, \tau\right) \mathrm{dz}\right)$, respectively, where $\alpha$ is the linewidth enhancement factor (LEF). It has been discussed in several articles that linewidth enhancement factor may vary in a large interval from the experimentally measured value of 0.1 up to giant values of 60 in QDs. The set of Eqs. 8-12 with the defined initial conditions cannot be solved in a closed form but it can only be solved numerically. For this purpose, the optical pulses and the SOAs have been divided in many small segments in time and distance, respectively, and solutions have been obtained stepwise both in time and space for the temporal gain and phase changes experienced by the clock pulses in the two arms of the interferometer. These are required to calculate the characteristics of the switched-out clock pulses at the transmission and reflection port of the interferometer, expressed by the equations $[25,27,28,38]$

$$
\begin{aligned}
& P_{\text {XOR }}=P_{\text {probe }}\left\{k_{1} k_{2} G_{A}(t)+\left(1-k_{1}\right)\left(1-k_{2}\right) G_{B}(t)-2 \sqrt{k_{1} k_{2}\left(1-k_{1}\right)\left(1-k_{2}\right) G_{A}(t) G_{B}(t)}\right\} \times \cos \left[\varphi_{A}-\varphi_{B}\right] \\
& P_{\text {cross }}=P_{\text {probe }}\left\{k_{1} k_{2} G_{A}(t)+\left(1-k_{1}\right)\left(1-k_{2}\right) G_{B}(t)+2 \sqrt{k_{1} k_{2}\left(1-k_{1}\right)\left(1-k_{2}\right) G_{A}(t) G_{B}(t)}\right\} \times \cos \left[\varphi_{A}-\varphi_{B}\right]
\end{aligned}
$$

$P_{\mathrm{XOR}}, P_{\mathrm{CROSS}}$ are the transmission and reflection functions.

$$
\begin{gathered}
g\left(h w_{\text {signal, prob }}\right)=g_{\text {max }}(2 f-1) \\
\alpha_{\text {abs }}=\alpha_{\text {max }}(1-2 h)+\alpha_{\text {int }}, S(z, \tau)=\frac{p(z, \tau)}{A_{\text {eff }} V_{g} h v}, p(z, t)=\sum_{i=1}^{n} P_{\text {max }} \exp \left(\frac{-4 \ln 2(t-n T)^{2}}{\tau_{\text {FWHM }}^{2}}\right)
\end{gathered}
$$

The wavelengths of signal, probe, and CP are considered to be: $\lambda_{s}=1.56 \mu \mathrm{m}, \lambda_{p}=1.53 \mu \mathrm{m}$ and $\lambda_{c}=1.31 \mu \mathrm{m}$. For the following structure parameters [3,35,36, 37,39]:

$$
\begin{aligned}
& g_{\max }=11.5 \mathrm{~cm}^{-1}, \alpha_{\mathrm{int}}=3 \mathrm{~cm}^{-1}, N_{Q}=5 \times 10^{10} \mathrm{~cm}^{-2}, L_{w}=0.25 \mu \mathrm{m}, \\
& \tau_{w 2}=3 \mathrm{ps}, \tau_{2 w}=1 \mathrm{~ns}, \tau_{w R}=0.2 \mathrm{~ns}, \tau_{21}=0.16 \mathrm{ps}, \tau_{12}=1.2 \mathrm{ps}, \tau_{1 R}=0.4 \mathrm{~ns}, \\
& W=10 \mu \mathrm{m}, \Gamma=3 \times 10^{-2}, \alpha_{\max }=10 \mathrm{~cm}^{-1}, \alpha_{\mathrm{LEF}}=1, W=10 \mu \mathrm{m}, \Gamma=3 \times 10^{-2}, \alpha_{\max }=10 \mathrm{~cm}^{-1}
\end{aligned}
$$

\section{Simulation results and discussion}

Achieving high-speed signal processing, as mentioned earlier, depends strongly on WL to ES and ES to GS relaxation times. However, $\tau_{21}$ is not a limiting factor in the operation of con- 
ventional QD-SOAs for $200 \mathrm{~Gb} / \mathrm{s}$. According to the reported results [1] pertaining to this case, $\tau_{21}$ is $160 \mathrm{fs}$ because of longer WL to ES relaxation time. However, this parameter can be important in achieving high-speed operation in the proposed approach as a higher limit. Increasing the $\tau_{21}=0.4$ ps relaxation time and consequence $\tau_{12}\left(\tau_{12}=\tau_{21} \exp \left[\left(E_{2}-E_{1}\right) / K_{B} T\right]\right)$ will decrease the quality factor for XOR and AND output. $K_{B}$ is the Boltzmann constant, $T$ is the absolute temperature, and $E_{2}-E_{1}$ is the energy separation between the ES and GS. Numerically calculated results are illustrated in the following figures:

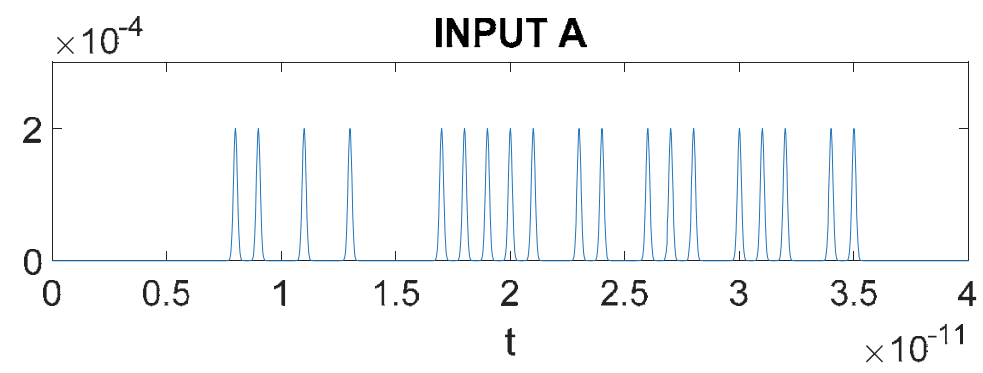

(a)

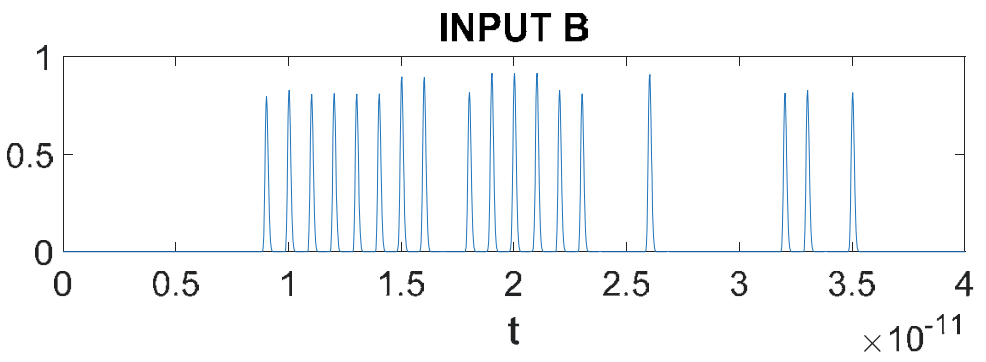

(b)

Figure 10. (a). Input waveforms of all-optical half-adder with input data stream A. (b). Input waveforms of all-optical half-adder with input data stream $B$.

Absorption of the $\mathrm{CP}$ will populate the conduction band excitation state (CBES) and hence the recovery process will accelerate, and the recovery process is much faster compared with the state where no $\mathrm{CP}$ is applied in the same input power and injected current.

At $2 \mathrm{~Tb} / \mathrm{s}$ bit sequence, the population variation cannot reach the final population value but still varies with relatively high amplitude.

At both bit rates of 1 and $2 \mathrm{~Tb} / \mathrm{s}$, the oscillation of ES and GS completely follows the input signal variation. The simulated output waveforms are shown in Fig. 13 (a) and (b), respectively, where Fig. 13 (a) illustrates the output sum-bit and 13 (b) shows output carry-bit. Fig. 13 (c) shows that the value of ER is decreased as this relaxation time is increased to 0.4 ps. With increase in the bit-rate value from $200 \mathrm{~Gb} / \mathrm{s}$, the $\mathrm{ER}=10 \log \left(\frac{P_{\mathrm{MIN}}^{1}}{P_{\mathrm{MAX}}^{0}}\right)$ is decreased as well. 


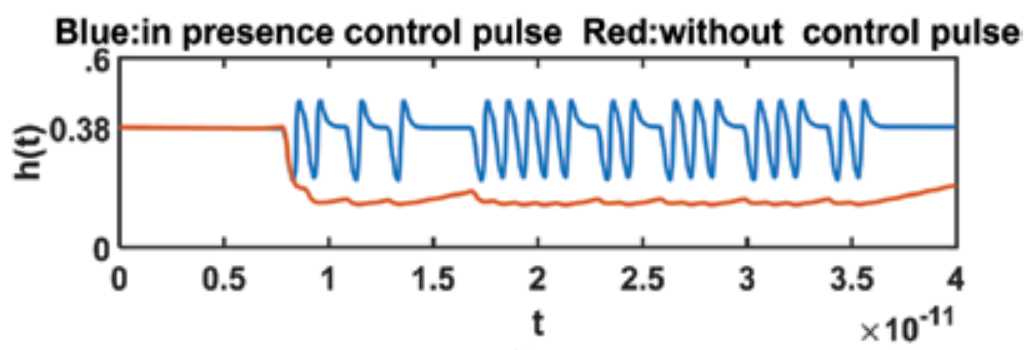

(a)

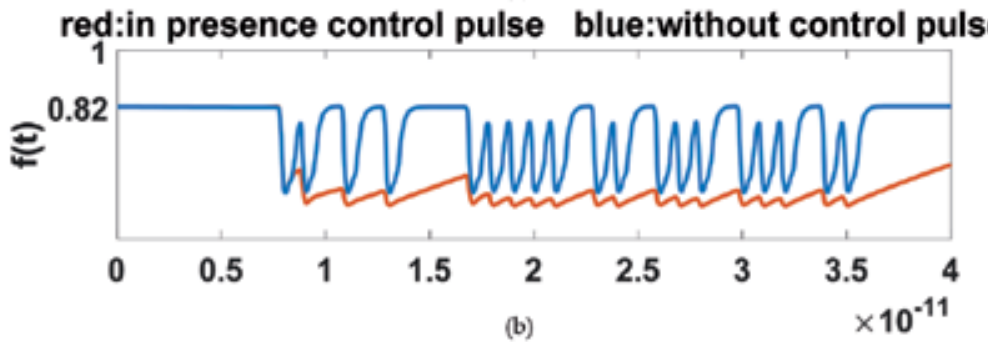

Figure 11. (a). Electron state occupation probabilities of ES, $h(t)$ with and without $\mathrm{CP}$ at $1 \mathrm{~Tb} / \mathrm{s}$. The bias current is 50 $\mathrm{mA}$, input signal, $\mathrm{CP}$, and probe signal powers are $200 \mathrm{UW}, 250 \mathrm{UW}$, and $2 \mathrm{UW}$, respectively. (b). Electron state occupation probabilities of ES, $f(t)$ with and without $\mathrm{CP}$ at $1 \mathrm{~Tb} / \mathrm{s}$. The bias current is $50 \mathrm{~mA}$, input signal, $\mathrm{CP}$, and probe signal powers are $200 \mathrm{UW}, 250 \mathrm{UW}$, and $2 \mathrm{UW}$, respectively.

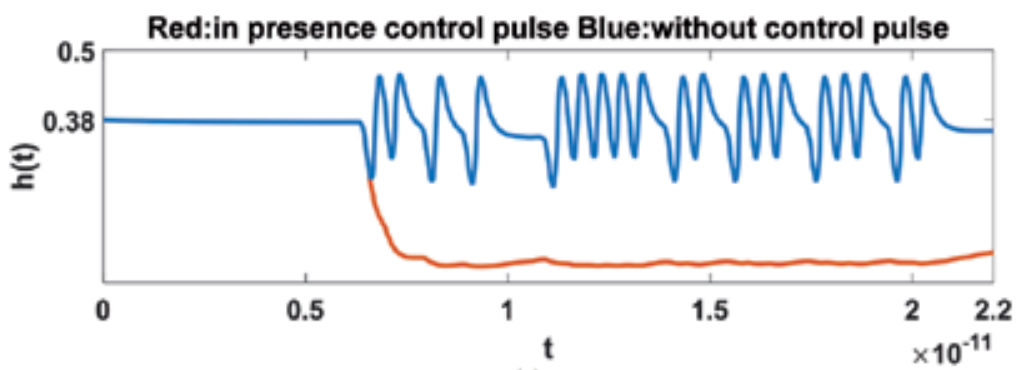

(a)

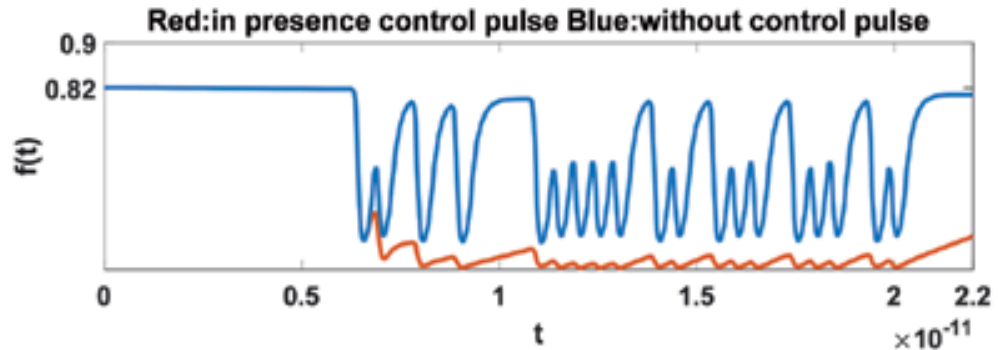

(b)

Figure 12. (a). Electron state occupation probabilities of ES, $h(t)$ with and without $\mathrm{CP}$ at $2 \mathrm{~Tb} / \mathrm{s}$. The bias current is 50 $\mathrm{mA}$, input signal, $\mathrm{CP}$, and probe signal powers are $200 \mathrm{UW}, 250 \mathrm{UW}$, and $2 \mathrm{UW}$, respectively. (b). Electron state occupation probabilities of ES, $f(t)$ with and without $\mathrm{CP}$ at $2 \mathrm{~Tb} / \mathrm{s}$. The bias current is $50 \mathrm{~mA}$, input signal, $\mathrm{CP}$, and probe signal powers are $200 \mathrm{UW}, 250 \mathrm{UW}$, and $2 \mathrm{UW}$, respectively. 


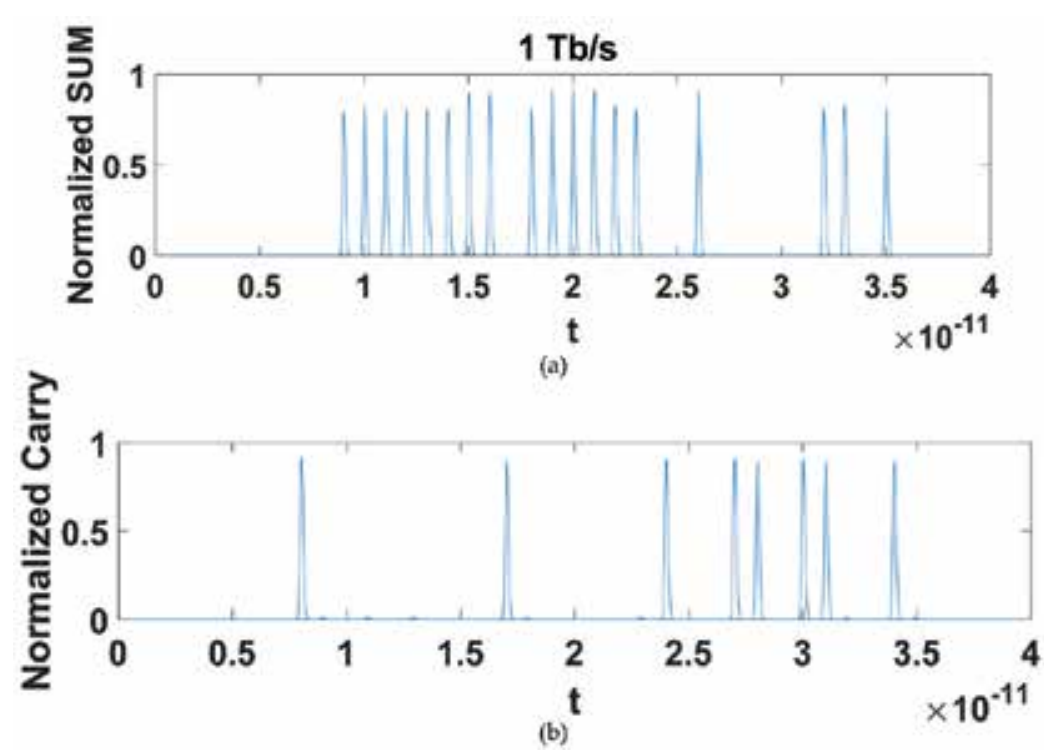

Figure 13. (a). Output waveforms of all-optical half-adders, where output sum-bit at $1 \mathrm{~Tb} / \mathrm{s}$, the results describe that the pattern effect is negligible at $1 \mathrm{~Tb} / \mathrm{s}$. (b). Output waveforms of all-optical half-adders, where output carry-bit at $1 \mathrm{~Tb} / \mathrm{s}$.

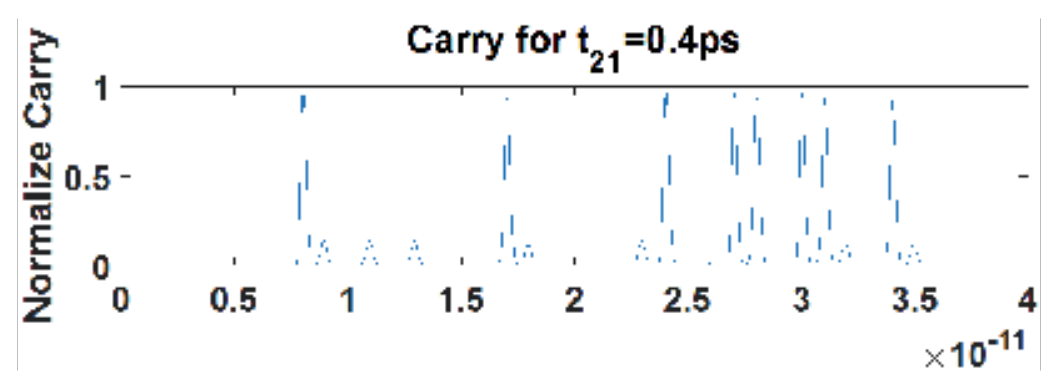

Figure 14. Output waveforms of all-optical half-adder, where output carry-bit at $1 \mathrm{~Tb} / \mathrm{s}$ with $\tau_{21}=0.4 \mathrm{ps}$.

The ER is changed with the change in the electron relaxation time during electron transport from the GS to the ES. Here, the value of ER is decreased in an exponential-like manner as this relaxation time is increased. Therefore, the transition time between GS and ES must be kept low as fast as possible. The device gain is determined by the carrier density of the QD ground state. As the carrier remains longer in GS before going to the ES level,,the transition rate from the ES to GS accelerates and the ES population rapidly decreases. Decrease in the ES population reduces the gain magnitude. The WL serves as the only recipient of the pump current, while QD's excited state serves as a carrier reservoir for the GS with ultrafast carrier relaxation to the latter, and their carrier density and transition rates can affect the device gain. Therefore, the slow electron transition between ES and WL occurs, which leads to the slowing down of the cross-gain modulation process. Hence, the extinction ratio is decreased. 


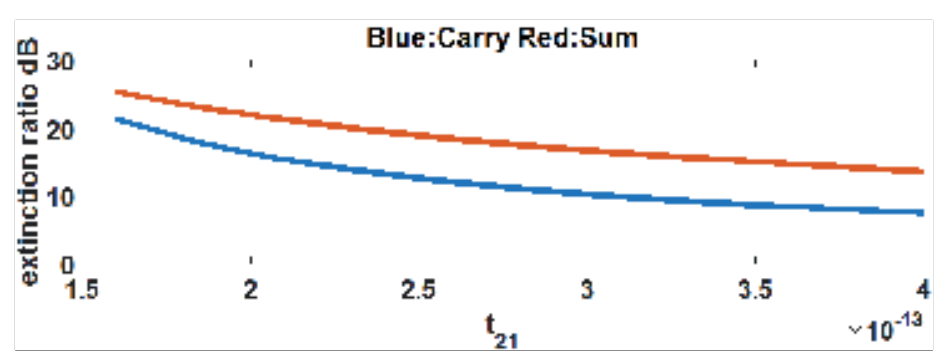

Figure 15. Variation of ER with electron relaxation time from the ES to the GS for input bit sequences at $1 \mathrm{~Tb} / \mathrm{s}$.

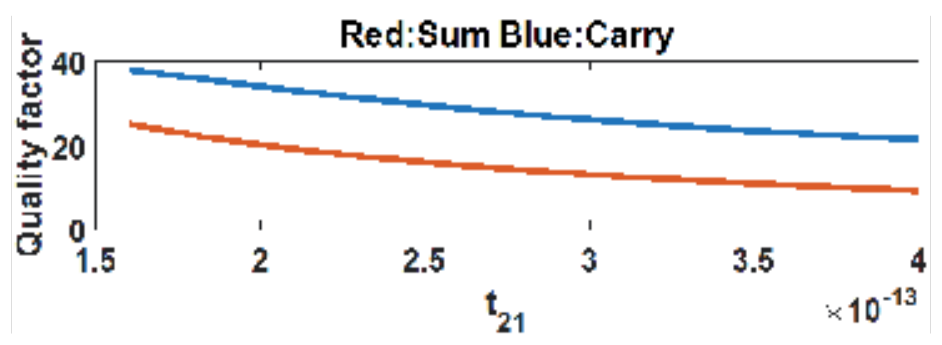

Figure 16. Quality factor with electron relaxation time from the ES to the GS for input bit sequences at $1 \mathrm{~Tb} / \mathrm{s}$.

Fig. 17 shows that the ER is very sensitive to the variations of the electron relaxation time from the ES to the GS since the slope of the curve is decreased in an exponential-like manner as this relaxation time is increased, finally becoming smoother near the left edge of the diagram. So the transition time between ES and GS must be kept below this limit and ideally be as fast as possible.

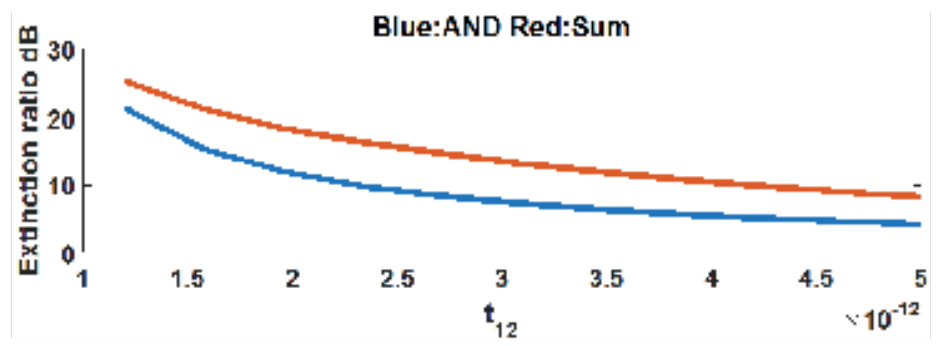

Figure 17. Variation of ER with electron relaxation time from the GS to the ES for input bit sequences at $1 \mathrm{~Tb} / \mathrm{s}$.

Fig. 18 illustrates the effect of sum with peak power of the input data signals on the ER. The characteristic of the curve is that the ER increases with power.

Fig. 19 illustrates the effect of carry with peak power of the input data signals on the ER. The characteristic of the curve is that the ER is increased with power up to a certain value after that it is decreased. 


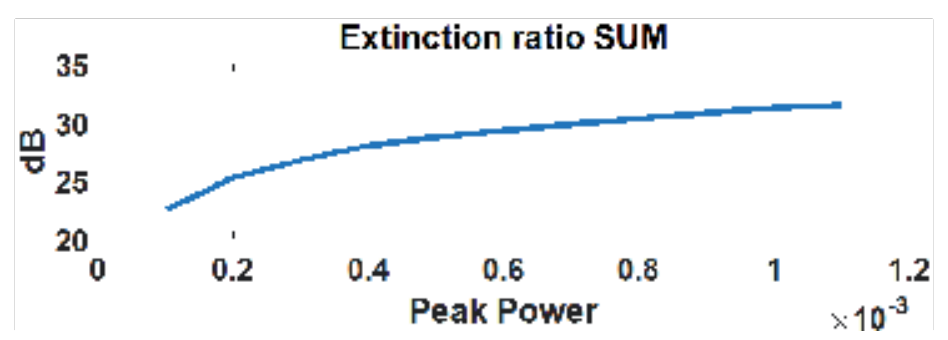

Figure 18. Variation of extinction ratio (ER) with peak data power for sum-bit, keeping other parameters fixed.

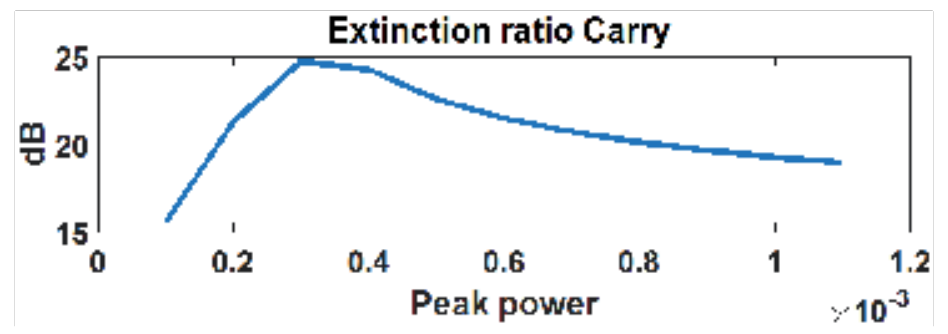

Figure 19. Variation of extinction ratio (ER) with peak data power for carry bit, keeping other parameters fixed.

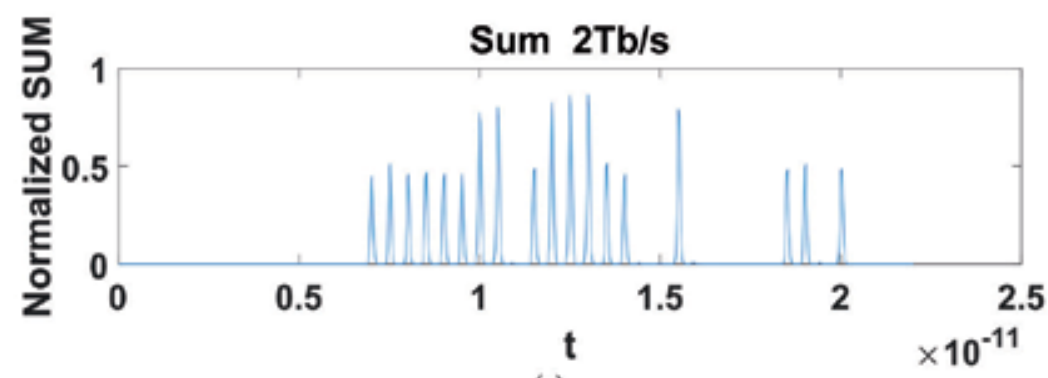

(a)

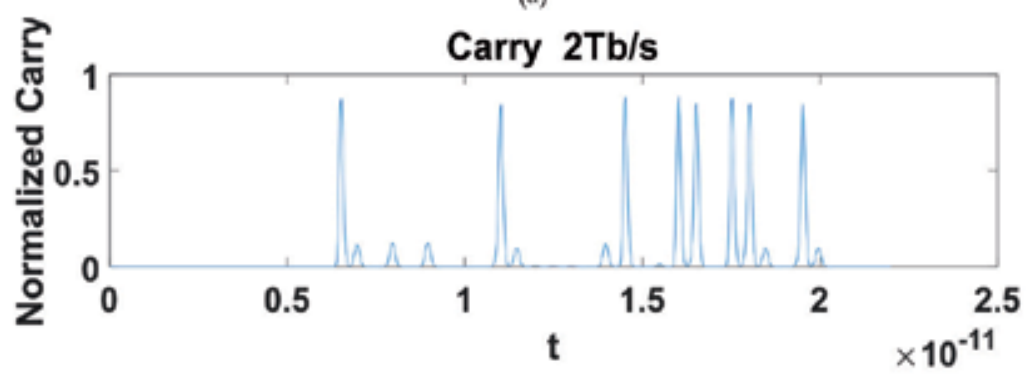

(b)

Figure 20. (a). Output waveforms of all-optical half-adder, sum-bit at $2 \mathrm{~Tb} / \mathrm{s}$. (b). Output waveforms of all-optical halfadder, carry-bit at $2 \mathrm{~Tb} / \mathrm{s}$.

The quality factor and ER are decreased with bit rate since at $2 \mathrm{~Tb} / \mathrm{s}$ bit sequence, the population variation cannot reach the final population value but still varies with relatively high amplitude. 


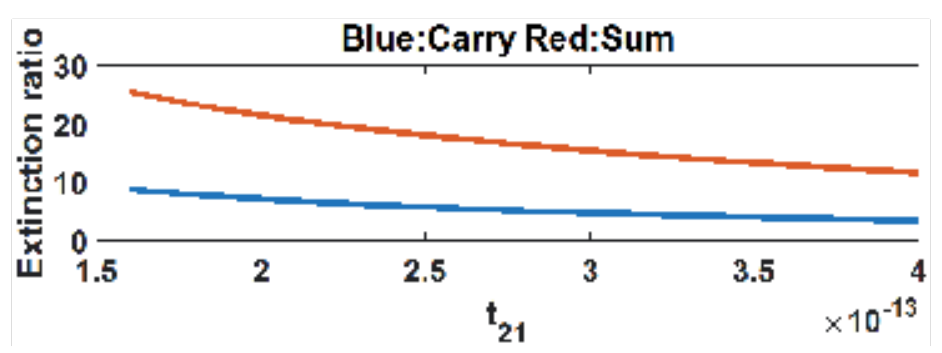

Figure 21. Variation of ER with electron relaxation time from the ES to the GS for input bit sequences at $2 \mathrm{~Tb} / \mathrm{s}$.

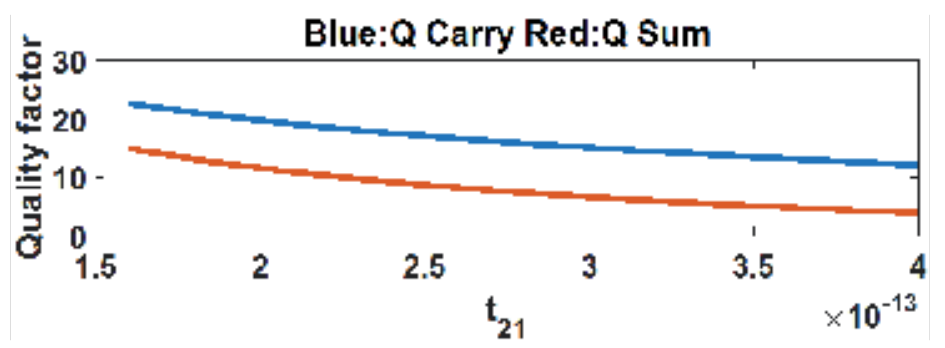

Figure 22. Quality factor with electron relaxation time from the ES to the GS for input bit sequences at $2 \mathrm{~Tb} / \mathrm{s}$.

\section{Conclusion}

A novel model of ultrafast all-optical half-adder using two QD-SOAs-based Mach-Zehnder interferometer was theoretically investigated and demonstrated. Numerically simulated results confirming the described method are also given in this chapter. The variation of ES to GS relaxation time on the ER and $Q$-factor at the output has been thoroughly investigated. We enhanced the bit rate, $Q$ factor, and extinction ratio parameters for the half-adder. We introduced theoretical approaches to compensate the slow-carrier transition relaxation time from WL to ES (using XGM effect) which is the main limit to achieve higher speeds in QD-SOAs. It is concluded that the proposed approach accelerates the recovery process of the SOA. Applying a CP to the two-energy level QD at certain times enables the QD-SOA-MZI-based halfadder to operate under 1 and $2 \mathrm{~Tb} / \mathrm{s}$ input bit sequences. This capability of control-pulseassisted QD-SOA is promising for ultrahigh-speed all-optical logic gates, all-optical switching, and processing. The model can be extended for studying more complex all-optical circuits of enhanced functionality in which this proposed circuit developed in this paper may be assumed as the basic building block. 


\section{Author details}

Khalil Safari ${ }^{1}$, Ali Rostami ${ }^{1,2^{*}}$, Ghasem Rostami ${ }^{2}$ and Mahboubed Dolatyari ${ }^{2}$

*Address all correspondence to: rostami@tabrizu.ac.ir

1 Photonics and Nanocrystal Research Lab. (PNRL), Faculty of Electrical and Computer Engineering, University of Tabriz, Tabriz, Iran

2 SP-EPT Labs., ASEPE Company, Industrial Park of Advanced Technologies, Tabriz, Iran

\section{References}

[1] Y. Ben-Ezra, B. I. Lembrikov, and M. Haridim, "Ultrafast all-optical processor based on quantum-dot semiconductor optical amplifiers," IEEE J. Quantum Electron., vol. 45, no. 1, pp. 34-41, 2009.

[2] B. Dai, S. Shimizu, X. Wang, and N. Wada, "Simultaneous all-Optical half-adder and half-subtracter based on two semiconductor optical amplifiers," IEEE Photonics Technol. Lett., 2012.

[3] D. K. Gayen, D. Kumar, and T. Chattopadhyay, "Designing of optimized all-optical half adder circuit using single quantum-dot semiconductor optical amplifier assisted Mach-Zehnder interferometer," J. Lightwave Technol., vol. 31, no. 12, pp. 2029-2035, 2013.

[4] A. Rostami, H. B. A. Nejad, R. M. Qartavol, and H. R. Saghai, "Tb/s optical logic gates based on quantum-dot semiconductor optical amplifiers," IEEE J. Quantum Electron., vol. 46, no. 3, pp. 354-360, 2010.

[5] L. M. Sa, H. Silva, P. Andre, and R. Nogueira, "Simulation performance of all-optical logic gate XOR at $40 \mathrm{Gbit} / \mathrm{s}$ using quantum-dot SOAs," 2011 IEEE EUROCON - International Conference on Computer as a Tool, 2011.

[6] H. L. Minh, F. Z. Ghassemlooy, and W. P. Ng, "All-optical flip-flop based on a symmetric Mach-Zehnder switch with a feedback loop and multiple forward set/reset signals," Opt. Eng., vol. 46, no. 4, pp. 40501-40503, 2007

[7] H. Sun, Q. Wang, H. Dong, and N. K. Dutta, "XOR performance of a quantum dot semiconductor optical amplifier based Mach-Zehnder interferometer," Opt. Exp., vol. 13, no. 6, pp. 1892-1899, 2005.

[8] R. Clavero, F. Ramos, J. M. Martinez, and J. Marti, "All-optical flip-flop based on a single SOA-MZI," IEEE Photonics Technol. Lett., vol. 17, no. 4, pp. 843-845, 2005.

[9] S. Diez, E. Hilliger, M. Kroh, C. Schmidt, C. Schubert, H. G. Weber, L. Occhi, L. Schares, G. Guekos, and L. K. Oxenloewe, "Optimization of SOA based Sagnac inter- 
ferometer switches for demultiplexing to 10 and 40 Gbit/s," Opt. Commun., vol. 189, no. 4-6, pp. 241-249, 2001.

[10] W. Hong, D. Huang, and G. Zhu, "Switching window of an SOA loop mirror with SOA sped-up by a CW assist light at transparency wavelength," Opt. Commun., vol. 238, no. 1-3, pp. 151-156, 2004.

[11] K. E. Zoiros, J. Vardakas, T. Houbavlis, and M. Moyssidis, "Investigation of SOA-assisted Sagnac recirculating shift register switching characteristics," Int. J. Light Electron Opt., vol. 116, no. 11, pp. 527-541, 2005.

[12] J. N. Roy and D. K. Gayen, "Integrated all-optical logic and arithmetic operations with the help of TOAD based interferometer device-alternative approach," Appl. Opt., vol. 46, no. 22, pp. 5304-5310, 2007.

[13] S. J. Savage, B. S. Robinson, S. A. Hamilton, and E. P. Ippen, “All-optical pulse regeneration in an ultrafast nonlinear interferometer with faraday mirror polarization stabilization," Opt. Lett., vol. 28, no. 1, pp.13-15, 2003.

[14] D. Gayen, A. Bhattacharyya, and J. Roy, "Ultrafast all-optical half adder using quantum-dot semiconductor optical amplifier-based Mach-Zehnder interferometer," J. Lightwave Technol., 2012.

[15] D. Li, X. Zhang, and D. Huang, "Novel all-optical format conversion using an ultrafast nonlinear interferometer at 10-40 Gbit/s," Microw. Opt. Technol. Lett., vol. 49, no. 3, pp. 508-510, 2007.

[16] K. E. Zoiros, P. Avramidis, and C. S. Koukourlis, "Performance investigation of semiconductor optical amplifier based ultrafast nonlinear interferometer in nontrivial switching mode," Opt. Eng., vol. 47, no.11, pp. 115006-115011, 2008.

[17] T. Siarkos and K. E. Zoiros, "Performance of single semiconductor optical amplifier based ultrafast nonlinear interferometer with clock control signals timing deviation in dual rail switching mode," Opt. Eng., vol. 48, no. 8, pp. 85004-85012, 2009.

[18] L. Han, H. Wen, H. Zhang, and Y. Guo, “All-optical wavelength conversion for polarization shift keying signal based on four-wave mixing in a semiconductor optical amplifier," Opt. Eng., vol. 46, no. 9, pp. 090501-090503, 2007.

[19] J. H. Kim, Y. T. Byun, Y. M. Jhon, S. Lee, D. H. Woo, and Sun Ho Kim. "All-optical half adder using semiconductor optical amplifier based devices,"Optics Commun., vol. 218, no. 4, pp. 345-349, 2003

[20] [20]Z. Chen, "Simple novel all-optical half adder," Opt. Eng., vol. 49, no.4, pp. 043201-043206, 2010

[21] [21]J. H. Kim, Y. T. Byun, Y. M. Jhon, S. Lee, D. H. Woo, and S. H. Kim, "All-optical half adder using semiconductor optical amplifier based devices," Opt. Commun., vol. 218, no. 4-6, pp. 345-349, 2003. 
[22] S. H. Kim, J. H. Kim, J. W. Choi, C.W. Son, Y. T. Byun, Y. M. Jhon, S. Lee, D. H. Woo, and S. H. Kim, "All-optical half adder using cross-gain modulation in semiconductor optical amplifiers," Opt. Exp., vol. 14, no. 22, pp. 10693-10698, 2006.

[23] P. L. Li, D. X. Huang, X. L. Zhang, and G. X. Zhu, "Ultrahigh speed all-optical half adder based on four-wave mixing in semiconductor optical amplifier," Opt. Exp., vol. 14, no. 24, pp. 11839-11847, 2006.

[24] P. Phongsanam, S. Mitatha, C. Teeka, and P. P. Yupapin, "All optical half adder/ subtractor using dark-bright soliton conversion control," Microw. Opt. Technol. Lett., vol. 53, no. 7, pp. 1541-1544, 2011.

[25] J. W. M. Menezes, W. B. Fraga, A. C. Ferreira, G. F. Guimaraes, A. F. G. F. Filho, C. S. Sobrinho, and A. S. B. Sombra, "All-optical half adder using all-optical XOR and AND gates for optical generation of "Sum" and "Carry"," Fiber Integr. Opt., vol. 29, no. 4, pp. 254-271, 2010.

[26] R. P. Schreieck, M. H. Kwakernaak, H. Jackel, and H. Melchior, "All-optical switching at multi-100-Gbit/s data rates with Mach-Zehnder interferometer switches," IEEE J. Quantum Electron., vol. 38, no. 8, pp. 1053-1061, 2002.

[27] A. Kumar, S. Kumar, and S. K. Raghuwanshi, "Implementation of full-adder and fullsubtractor based on electro-optic effect in Mach-Zehnder interferometers," Opt. Commun., 2014.

[28] A. Rostami, "Applications and Functionalities, "Eng. Mater, 2011.

[29] S. Nakamura, Y. Ueno, K. Tajima, J. Sasaki, T. Sugimoto, T. Kato, T. Shimoda, M. Itoh, H. Hatakeyama, T. Tamanuki, and T. Sasaki, "Demultiplexing of 168-Gb/s data pulses width a hybrid-integrated symmetric Mach-Zehnder all-optical switch," IEEE Photon. Technol. Lett., vol. 12, no. 5, pp. 425-427, 2000.

[30] D. K. Gayen, A. Bhattachryya, T. Chattopadhyay, and J. N. Roy, “Ultrafast all-optical half adder using quantum-dot semiconductor optical amplifier based Mach-Zehnder interferometer," J. Lightwave Technol., vol. 30, no. 21, pp. 3387-3393, 2012.

[31] [31]A. Rostami, H. Baghban, R. Maram, "Nanostructure Semiconductor Optical Amplifiers," Berlin, Germany: Springer-Verlag, 2011.

[32] J. Y. Kim, J. M. Kang, T. Y. Kim, and S. K. Han, "All-optical multiple logic gates with XOR, NOR, OR, and NAND functions using parallel SOA-MZI structures: Theory and experiment," J. Lightwave Technol., vol. 24, no. 9, pp. 3392-3399, 2006.

[33] H. Sun, Q. Wang, H. Dong, and N. K. Dutta, "XOR performance of a quantum-dot semiconductor optical amplifier based Mach-Zender interferometer," Opt. Exp., vol. 13, no. 6, pp. 1892-1899, 2005. 
[34] E. Dimitriadou and K. E. Zoiros, “On the feasibility of ultrafast all-optical NAND gate using single quantum-dot semiconductor optical amplifier-based Mach-Zehnder interferometer," Opt. Laser Technol., vol. 44, no. 6, pp. 1971-1981, 2012.

[35] E. Dimitriadou and K. E. Zoiros, "Proposal for all-optical NOR gate using single quantum-dot semiconductor optical amplifier-based Mach-Zehnder interferometer," Opt. Commun., vol. 285, pp.1710-1716, 2012.

[36] E. Dimitriadou and K. E. Zoiros, "On the design of ultrafast all-optical NOT gate using quantum-dot semiconductor optical amplifier based Mach-Zehnder interferometer," Opt. Laser Technol., vol. 44, pp.600-607, 2012.

[37] J. Wang, Y. Jiao, R. Bonk, W. Freude, and J. Leuthold, "Regenerative properties of bulk and quantum-dot SOA based all-optical Mach-Zehnder interferometer DPSK wavelength converters," in Proc. Conf. Photon. Switching, October 16-18, 2006, pp. 13.

[38] H. Han, M. Zhang, P. Ye, and F. Zhang, "Parameter design and performance analysis of an ultrafast all-optical XOR gate based on quantum-dot semiconductor optical amplifiers in nonlinear Mach-Zehnder interferometer," Opt. Commun., vol. 281, no. 20, pp. 5140-5145, 2008.

[39] M. Sugawara, H Ebe, N. Hatori, M. Ishida, Y. Arakawa, T. Akiyama, K. Otsubo, and Y. Nakata, "Theory of optical signal amplification and processing by quantum-dot semiconductor optical amplifiers," Phys. Rev., B 69, 235332-1-39 (2004)

[40] O. Qasaimeh, "Characteristics of cross-gain (XG) wavelength conversion in quantum dot semiconductor optical amplifier," IEEE Photon.Technol. Lett., vol. 16, no. 2, pp. 542-544, 2004.

[41] T. W. Berg, S. Bischoff, I. Magnusdottir, and J. Mark, “Ultrafast gain recovery and modulation limitations in self-assembled quantum-dot devices," IEEE Photon. Technol. Lett., ser. 6, vol. 13, pp. 541-543, 2001.

[42] T. W. Berg and J. Mark, "Saturation and noise properties of quantum-dot optical amplifiers," IEEE J. Quantum Electron., vol. 40, no. 11, pp. 1527-1539, 2004.

[43] J. Wang, Y. Jiao, R. Bonk, W. Freude, and J. Leuthold, "Regenerative properties of bulk and quantum-dot SOA based all-optical Mach-Zehnder interferometer DPSK wavelength converters," in Proc. Conf. Photon. Switching, October 16-18, 2006, pp. $1-3$.

[44] C. I. Sandall, C. L. Walker, P. M. Smowton, D. J. Mowbray, H. Y. Liu, and M. Hopkinson, "Measurement of modal absorption, gain and recombination in p-doped and intrinsic quantum dot structures," Proc. IEE Optoelectron., vol. 153, no. 6, pp. 316-320, 2006. 

Chapter 5

\title{
Semiconductor Optical
}

\section{Amplifier (SOA)-Based Amplification of \\ Intensity-Modulated Optical Pulses \\ - Deterministic Timing Jitter and \\ Pulse Peak Power Equalization Analysis}

\author{
T. Alexoudi, G.T. Kanellos, S. Dris, D. Kalavrouziotis, P. Bakopoulos, \\ A. Miliou and N. Pleros
}

Additional information is available at the end of the chapter

http://dx.doi.org/10.5772/61712

\begin{abstract}
During the last few years, large-scale efforts towards realizing high-photonic integration densities have put SOAs in the spotlight once again. Hence, the need to develop a complete framework for SOA-induced signal distortion to accurately evaluate a system's performance has now become evident. To cope with this demand, we present a detailed theoretical and experimental investigation of the deterministic timing jitter and the pulse peak power equalization of SOA-amplified intensity-modulated optical pulses. The deterministic timing jitter model relies on the pulse mean arrival time estimation and its analytic formula reveals an approximate linear relationship between the deterministic timing jitter and the logarithmic values of intensity modulation when the SOA gain recovery time is faster than the pulse period. The theoretical analysis also arrives at an analytic expression for the intensity modulation reduction (IMR), which clearly elucidates the pulse peak power equalization mechanism of SOA. The IMR analysis shows that the output intensity modulation depth is linearly related to the respective input modulation depth of the optical pulses when the gain recovery time is faster than the pulse period. This novel theoretical platform provides a qualitative and quantitative insight into the SOA performance in case of intensity-modulated optical pulses.
\end{abstract}

Keywords: Deterministic timing jitter, Pulse peak power equalization, Intensity modulation reduction, Semiconductor optical amplifier, Modulation depth index 


\section{Introduction}

Semiconductor optical amplifiers (SOAs) have long been the subject of considerable research interest, mainly exploiting their nonlinear properties to provide fast all-optical signal processing [1], such as high-speed wavelength conversion (WC) [2, 3], bitwise logic operations [4-6], and signal regeneration [7]. The broad-scale efforts towards realizing high photonic integration densities have, however, put the use of SOAs as amplification elements in the spotlight once again, since any alternative integrated amplifier competitor [8] lags far behind in terms of integration maturity. SOAs currently emerge as the preeminent on-chip amplifier solution and their reintroduction in the toolbox of the optical network designer is now evident in many key network subsystems. As a result, multiple demonstrations of SOAs performing as pure amplifier stages $[9,10]$ or as ON-OFF gating elements [11], where amplification occurs in the ON state, have been presented. Their ubiquitous use spans diverse network segments, enabling leading edge applications that extend from metro [11] to access network environments [10] and to on-chip or on-board datacom systems [9].

A concerted research effort on SOA-based devices, spanning the last 20 years, has unraveled most of their underlying amplification secrets, addressing a variety of linear and nonlinear phenomena and their impact on a system's performance [12]. Pulse-shaped asymmetry owing to SOA saturation effects, for example, has been one of the key findings and has been extensively studied for the past years [13]. However, it was only recently that a novel theoretical analysis correlated this behavior to SOA-induced deterministic timing jitter that optical pulses experience during the amplification process, also suggesting an analytic mathematical formula for its accurate estimation [14, 15]. On the other hand, amplitude modulation phenomena for SOA in-line amplification have been theoretically studied $[16,17]$ but the pulse peak power equalization properties of SOAs, although experimentally utilized in many cases [18-20], have never been expressed in an analytical form that would allow a straightforward estimation for any case of input signal. So far, the pulse peak power equalization properties of SOAs have been theoretically and experimentally investigated only for the SOA-based interferometric switches [20]. As a result, the proposed theoretical model cannot be applied for single SOA inline amplification cases, since it relies on cross-phase modulation (XPM) phenomena that take place in SOA-based interferometric devices. Although research efforts have shed plenty of light on the SOA-based amplification process during the last few years, a complete framework for SOA-induced signal distortion in case of intensity-modulated optical pulses, including both deterministic timing jitter and the intensity modulation reduction analysis, is still missing.

In order to fill the current gap in the system's performance assessment, we present here a holistic theoretical analysis for the SOA-based amplification process along with its experimental verification when intensity-modulated optical pulses are inserted into the amplifier. The aim of this chapter is to provide a systematic methodology on the origin, nature and quantification of SOA-induced deterministic timing jitter and pulse peak power equalization that intensity-modulated optical pulses experience during the amplification process. At first, an analytic formula for the pulse mean arrival time at the SOA exit is derived, providing a comprehensive picture of jitter origin and allowing for reliable estimation of the deterministic 
jitter induced during the SOA amplification. The theoretical analysis continues with an analytical mathematical expression of intensity modulation reduction induced by SOA amplification. More specifically, the output-versus-input modulation depth of the amplifier is examined for several saturation levels to thoroughly investigate the pulse peak power equalization capabilities of the SOA. The theoretical models are also experimentally verified with the obtained results proving good agreement between theory and experimental observations, in both cases. Moreover, the deterministic timing jitter analysis reveals an approximate linear relationship between jitter values and the logarithm of pulse peak power modulation. Both experimental and theoretical results show that deterministic timing jitter minimization can be achieved by operating the SOA in the strongly saturated region. On the other hand, pulse peak power equalization analysis indicates a linear dependence between the output and input modulation depth indices. In that case, results show that the amplifier yields higher intensity modulation reduction values when it is operated in the saturation regime and for increased SOA gain levels.

In this perspective, the following sections of the chapter have been organized so as to introduce the concept and provide the analytical theoretical framework of the SOA-induced deterministic timing jitter and the pulse peak power equalization properties for intensity-modulated optical pulses, as well as to describe the experimental setup along with the respective results obtained in each case and finally discuss potential extensions of the proposed theoretical models.

\section{Concept and theoretical analysis}

It is a well-known fact that intensity-modulated optical pulses will experience a pulse-shaped distortion and intensity modulation suppression when propagating through the SOA. The shift of the amplified pulse peak towards its rising edge owes to the higher gain that the leading edge of every incoming pulse experiences compared to the gain received by the trailing edge of the pulse [13]. This "center of gravity" deviation of the exiting optical pulse indicates a subsequent deviation of the mean pulse arrival time $T_{\text {MEAN }}$ at the SOA exit. These systematic signal asymmetries originating from steeper and more gradual pulse edges are the root cause of the deterministic timing jitter depending on input data characteristics. When pulses of unequal peak power arrive at the input of the SOA, each pulse is displaced by a different amount resulting in a mean arrival time deviation at the output. Thus, when intensitymodulated pulses are injected into the SOA, and assuming the pulse period is greater than the SOA-gain recovery time, the different peak power levels will generate different dips in the SOA gain that cause different pulse shifts, leading to varying timing jitter values.

Apart from the peak position deviation of the optical pulses, SOAs can also induce pulse peak power equalization of incoming intensity-modulated optical pulses. This can, in turn, yield in a reduction in intensity modulation of the optical pulses at the SOA output. Assuming, again, that the SOA gain recovery time is faster than the pulse period, the pulse peak power equalization originates from the amplification dissimilarities arising between the low and high pulse 
peak powers. A high gain is received by the lower peak-powered pulses whereas a lower gain is experienced by the higher peak-powered pulses, resulting in nearly power-equivalent amplified pulses obtained at the SOA exit. As a result, the modulation depth index of the input pulses will always be higher than the respective outputs of the amplifier pointing out an intensity modulation reduction of the exiting optical pulse stream.

The following analysis aims to provide a theoretical insight into the origin of the deterministic timing jitter and elucidate the pulse peak power equalization mechanism during the SOA amplification of intensity-modulated optical pulses. Considering the amplifier as a spatially concentrated device, the instantaneous amplifier gain $G(t)$ experienced by each pulse entering the SOA is expressed as [13, 20]:

$$
G(t)=1 /\left[1-\left(1-1 / G_{0}\right) \cdot \exp \left(-U_{\text {in }}(t) / U_{\text {sat }}\right)\right]
$$

where $G_{0}$ represents the SOA steady-state gain and $U_{\text {in }}(t)$ the accumulated injected pulse energy given by:

$$
U_{\text {in }}(t)=\int_{-\infty}^{t} P_{\text {in }}(t) \cdot d t
$$

and the $U_{\text {sat }}$ is the well-known saturation energy of the device. To this end, the output pulse exiting the SOA can be calculated as:

$$
P_{\text {out }}(t)=P_{\text {in }}(t) \cdot G(t)
$$

by defining $P_{\text {in }}(t)$ as the input pulse power.

\subsection{Deterministic timing jitter analysis}

Considering Gaussian pulses as input to the SOA, the input pulse power is defined as $P_{\text {in }}(t)=P_{p} \cdot \exp \left(-t^{2} / T_{0}^{2}\right)$ with peak power denoted as $P_{\mathrm{p}}$ and $1 / e$ pulsewidth equal to $T_{0}$. The mean arrival time $T_{\text {MEAN }}$ for every individual pulse is calculated [21, 22] as:

$$
T_{\text {MEAN }}=\frac{\int_{-\infty}^{+\infty} t \cdot P_{\text {out }}(t) \cdot d t}{U_{\text {total }}}=\frac{\int_{-\infty}^{+\infty} t \cdot P_{\text {in }}(t) \cdot G(t) \cdot d t}{\int_{-\infty}^{+\infty} P_{\text {in }}(t) \cdot G(t) \cdot d t}=\frac{A}{B}
$$

where $U_{\text {total }}$ is the total output pulse energy. By replacing $P_{\text {in }}(t)$ and $G(t)$ with their respective expressions, we obtain $A$ and $B$ in Eq. (5) and Eq. (6), respectively. 


$$
\begin{aligned}
& A=\int_{-\infty}^{+\infty} t \cdot P_{\mathrm{p}} \cdot \exp \left(-\frac{t^{2}}{T_{0}^{2}}\right) \cdot\left[1-\left(1-\frac{1}{G_{0}}\right) \cdot \exp \left(\frac{-P_{\mathrm{p}} \cdot \int_{-\infty}^{t} \exp \left(-\frac{t^{2}}{T_{0}^{2}}\right) \cdot d t}{U_{\mathrm{sat}}}\right)\right]^{-1} \cdot d t \\
& B=\int_{-\infty}^{+\infty} P_{\mathrm{p}} \cdot \exp \left(-\frac{t^{2}}{T_{0}^{2}}\right) \cdot\left[1-\left(1-\frac{1}{G_{0}}\right) \cdot \exp \left(\frac{-P_{\mathrm{p}} \cdot \int_{-\infty}^{t} \exp \left(-\frac{t^{2}}{T_{0}^{2}}\right) \cdot d t}{U_{\mathrm{sat}}}\right)\right]^{-1} \cdot d t
\end{aligned}
$$

By expanding $G(t)$ both in the numerator $A$ and in the denominator $B$ in a first-order Taylor series around the center position of the pulse at $t=0$, after some algebra, Eq. (4) becomes:

$$
T_{\text {MEAN }}=-\frac{T_{0}^{2} \cdot\left(1-\frac{1}{G_{0}}\right) \cdot\left[\frac{P_{\mathrm{p}}}{U_{\text {sat }}} \cdot \exp \left(-\frac{P_{\mathrm{p}}}{U_{\text {sat }}} \cdot \frac{T_{0 \cdot \sqrt{\pi}}}{2}\right)\right]}{2 \cdot\left[1-\left(1-\frac{1}{G_{0}}\right) \cdot \exp \left(-\frac{P_{\mathrm{p}}}{U_{\text {sat }}} \cdot \frac{T_{0} \cdot \sqrt{\pi}}{2}\right)\right]}
$$

Eq. (7) determines the mean arrival time $T_{\text {MEAN }}$ of every pulse as a function of its peak power, its time duration and of the SOA steady-state gain. In Figure 1, the $T_{\text {MEAN }}$ quantity is illustrated versus a pulse peak power range of $0 \mathrm{~mW}$ to $20 \mathrm{~mW}$, for three different SOA gain $G_{0}$ values and a pulsewidth of $20 \mathrm{ps}$. As the center of the pulse is assumed to be at $t=0$, the time shift induced by SOA amplification process will always be leftward. As a consequence, $T_{\text {MEAN }}$ will always be a negative quantity that will continuously decrease until reaching a saturation plateau [15].

In addition, according to Figure 1, the steepness of the slope of the $T_{\text {MEAN }}$ curve indicated two distinct areas for the $T_{\text {MEAN }}$ based on the peak power: area A, corresponding to the nonsaturated SOA gain regime, where the SOA still responds radically to input peak power resulting in enhanced timing jitter values for the amplified pulses, and area B, where the SOA operates in its strongly saturated gain region. In the last case, the curve of $T_{\text {MEAN }}$ decreases smoothly, mitigating in this way the differences of mean arrival time $T_{\text {MEAN }}$ compared to SOA operation in area A and leading to lower timing jitter values [15].

The monotonic slope of mean arrival time $T_{\text {MEAN }}$ implies that the lowest and highest values are obtained for the corresponding lowest and highest peak power input pulses [15]. In case the $P_{\mathrm{p}}$ values are within a finite set between minimum and maximum values, the peak-to-peak 


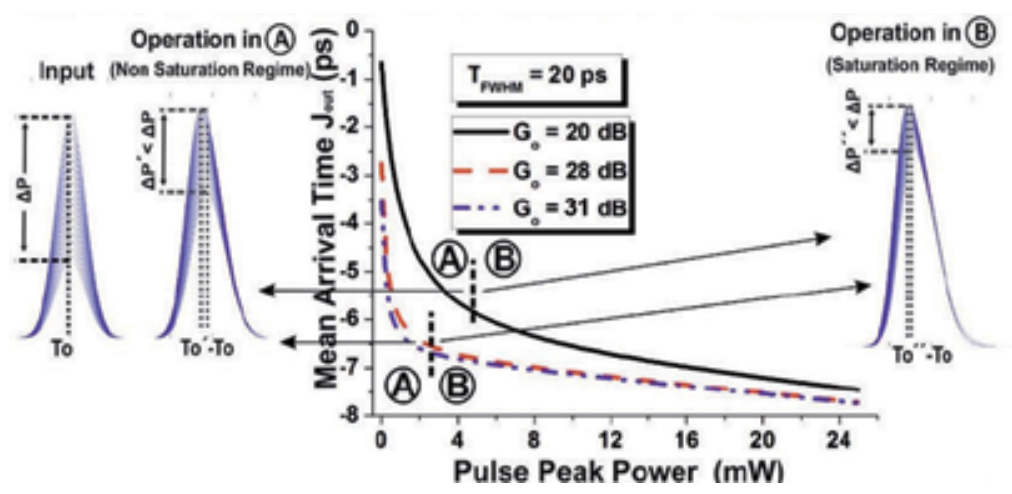

Figure 1. Theoretical mean arrival time $\left(T_{\text {MEAN }}\right)$ vs. pulse peak power values. Insets of the figure show the eyediagrams of a jitter-free intensity-modulated signal before entering the SOA amplifier and the output signals when the SOA operates in the nonsaturation (area A) and in the strongly saturated regime (area B), respectively.

deterministic timing jitter is determined as the difference between the respective minimum and maximum mean arrival time values $T_{\text {MEAN }}[15]$, as shown in Eq. (8):

$$
J_{\mathrm{pp}}^{D}=T_{\mathrm{MEAN}}^{\max }-T_{\mathrm{MEAN}}^{\min }
$$

where $T_{\text {MEAN }}^{\min }=T_{\text {MEAN }}\left(P_{\mathrm{p}}^{\min }\right)$ and $T_{\text {MEAN }}^{\max }=T_{\text {MEAN }}\left(P_{\mathrm{p}}^{\max }\right)$.

An interesting conclusion for deterministic timing jitter can be drawn by expressing the peak power $P_{\mathrm{p}}$ in logarithmic instead of linear scale. Figure 2 is a graphical representation of Eq. (7), showing the dependence of $T_{\text {MEAN }}$ on peak power values, when the latter is expressed in $\mathrm{dBm}$. As illustrated, the curved sections for peak power values lower than $-5 \mathrm{dBm}$, and higher than $0 \mathrm{dBm}$ can be very well approximated by second-degree polynomials.
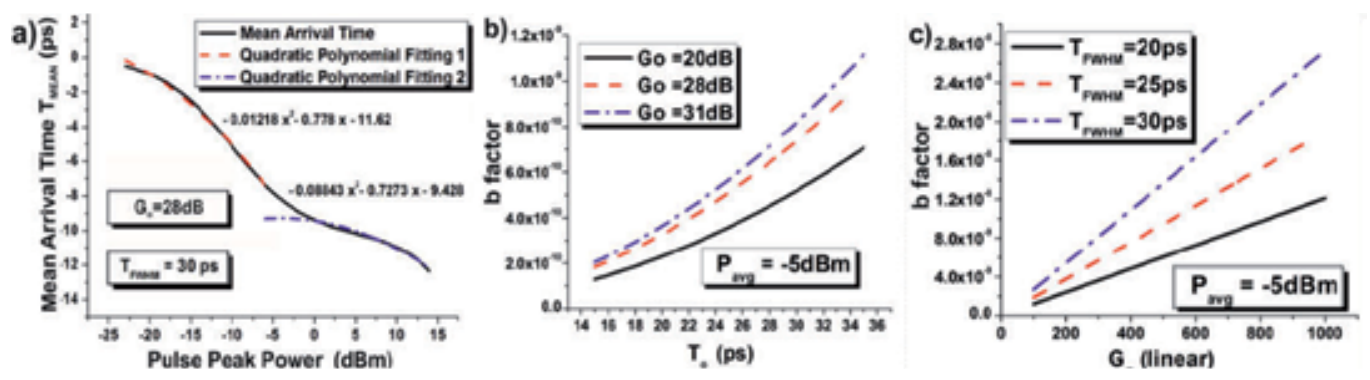

Figure 2. a) Mean arrival time ( $T_{\text {MEAN }}$ ) vs. pulse peak power expressed in $\mathrm{dBm}$ for Go $=28 \mathrm{~dB}$ and 30 -ps-long pulses. Dashed lines denote the fit of $T_{\text {MEAN }}$ with second-degree polynomials. Linearity of deterministic timing jitter denoted by $b$ factor of Eq. (12) versus (b) 1/e pulsewidth and (c) SOA gain levels, showing the dependence of $T_{\text {MEAN }}$ on peak power values, the latter expressed in $\mathrm{dBm}$. 
Thus, Eq. (7) can be expanded into a second-order Taylor series around a reference peak power $P_{\mathrm{REF}}$, again expressed in $\mathrm{dBm}$. Moreover, by substituting in Eq. (8), the values of $T_{\text {MEAN }}$ corresponding to the highest and lowest peak power pulses $P_{\mathrm{p}}^{\max }(\mathrm{dBm})$ and $P_{\mathrm{p}}^{\min }(\mathrm{dBm})$, respectively, the deterministic timing jitter can now be written as shown in Eq. (9):

$$
J_{P_{\mathrm{p}}}^{D}=\frac{1}{2 !} \cdot a \cdot\left[\left(P_{\mathrm{p}}^{\max }(\mathrm{dBm})-P_{\mathrm{REF}}(\mathrm{dBm})\right)^{2}-\left(P_{\mathrm{p}}^{\min }(\mathrm{dBm})-P_{\mathrm{REF}}(\mathrm{dBm})\right)^{2}\right]+b \cdot\left(P_{\mathrm{p}}^{\max }(\mathrm{dBm})-P_{\mathrm{p}}^{\min }(\mathrm{dBm})\right)
$$

where $a=\left.\frac{d^{2} J_{p_{p}}^{D}}{d P^{2}}\right|_{P=P_{R E F(A B m)}}$ and $\mathrm{b}=\left.\frac{d J_{p_{p}}^{D}}{d P}\right|_{P=P_{R E F(A B m)}}$ Eq. (9) can be further simplified using some straightforward algebra into:

$$
J_{p_{\mathrm{p}}}^{D}=\frac{1}{2 !} \cdot a \cdot \Delta P(\mathrm{~dB}) \cdot\left[P_{\mathrm{p}}^{\max }(\mathrm{dBm})+P_{\mathrm{p}}^{\min }(\mathrm{dBm})-2 \cdot P_{\mathrm{REF}}(\mathrm{dBm})\right]+b \cdot \Delta P(\mathrm{~dB})
$$

where $\Delta P=P_{\mathrm{p}}^{\max }-P_{\mathrm{p}}^{\min }$ is the intensity modulation expressed in $\mathrm{dB}$. By selecting the $P_{\mathrm{REF}}(\mathrm{dBm})$ value to be the mid-point between the minimum $P_{\mathrm{p}}^{\min }$ and maximum $P_{\mathrm{p}}^{\max }$ peak power level, so that $P_{\mathrm{p}}^{\max }(\mathrm{dBm})=P_{\mathrm{REF}}(\mathrm{dBm})+\Delta P(d B) / 2$. and $P_{\mathrm{p}}^{\min }(\mathrm{dBm})=P_{\mathrm{REF}}(\mathrm{dBm})-\Delta P(\mathrm{~dB}) / 2$, the quantity contained in the brackets becomes zero and the deterministic timing jitter expression turns into:

$$
J_{\mathrm{p}_{p}}^{\mathrm{D}}=b \cdot \Delta P(\mathrm{~dB})
$$

This formula reveals a linear relationship between deterministic timing jitter and intensity modulation with the linearity factor $b$ provided by Eq. (12).

$$
\begin{aligned}
& -T_{0}^{2} \cdot e^{\frac{P_{\mathrm{REF}}\left(\mathrm{dBm} \cdot 10^{-3}\right)}{10}} \cdot\left(G_{0}-1\right) . \\
& \left(2 \cdot G_{0}-2 \cdot G_{0} \cdot e^{\frac{T_{0} \cdot \sqrt{\pi} \cdot U_{\mathrm{sat}} \cdot e^{\frac{P_{\mathrm{REF}}\left(\mathrm{dBm} \cdot 10^{-3}\right)}{10}}}{2}}+G_{0} \cdot T_{0} \cdot \sqrt{\pi} \cdot U_{\mathrm{sat}} \cdot e^{\frac{P_{\mathrm{REF}} \cdot\left(\mathrm{dBm} \cdot 10^{-3}\right)}{10}+\frac{T_{0} \cdot \sqrt{\pi} \cdot U_{\mathrm{sat}} \cdot e^{\frac{P_{\mathrm{REF}}\left(\mathrm{dBm} \cdot 10^{-3}\right)}{10}}}{2}}-2\right) . \\
& \cdot\left(40 \cdot U_{\mathrm{sat}} \cdot\left(G_{0} \cdot e^{\frac{T_{0} \cdot \sqrt{\pi} \cdot U_{\mathrm{sat}} \cdot e^{\frac{P_{\mathrm{REF}}\left(\mathrm{dBm} \cdot 0^{-3}\right)}{10}}}{2}}-G_{0}+1\right)^{2}\right)^{-1}
\end{aligned}
$$


By plotting Eq. (12) for different pulsewidths and SOA gain levels as shown in Figure 2(b) and in Figure 2(c), respectively, the absolute value of $b$ increases with $T_{0}$ and $G_{0}$ for a given $P_{\mathrm{REF}}(\mathrm{dBm})$ value. This indicates that higher jitter values are obtained for higher pulsewidths and higher SOA gains when the same intensity modulation level and the same $P_{\mathrm{REF}}(\mathrm{dBm})$ values are used.

\subsection{Pulse peak power equalization analysis}

By defining $T$ as the bit period, $P_{p}$ as the average peak power value across the whole control signal sequence, $\Omega$ as the modulation frequency and $m$ as the modulation depth index, the peak power of each $k$-th individual pulse of an intensity-modulated clock pulse sequence entering the SOA is given by $P_{\mathrm{p}}^{k}=P_{\mathrm{p}} \cdot[1+m \cdot \cos (\Omega \cdot k \cdot T)]$. Since multilevel clock puls are considered to enter the SOA as input, the modulation depth index can be determined by their discrete levels. By substituting the intensity-modulated clock pulse sequence $P_{\mathrm{p}}^{k}$ in $(2), U_{\mathrm{in}}(t)$ is transformed as follows:

$$
U_{\text {in }}(t)=\int_{-\infty}^{t} P_{\text {in }}(t) \cdot d t=P_{\mathrm{p}} \cdot(1+m \cdot \cos (\Omega \cdot k \cdot T)) \cdot \int_{-\infty}^{t} a\left(t^{\prime}\right) \cdot d t^{\prime}
$$

where $a\left(t^{\prime}\right)$ denotes the pulse waveform. Eq. (1) shows that the gain saturates to a minimum value until the whole pulse energy has passed through the amplifier. However, it is assumed that the gain recovers back to its steady-state value before the next pulse enters the amplifier. As a result, Eq. (1) is valid for the whole bit sequence, allowing in this way for the replacement of the time-dependent integral $\int a\left(t^{\prime}\right) \cdot d t^{\prime}$ contained in Eq. (13) with a time-independent constant value $A$ that corresponds to the total area contained in the pulse waveform [20]. To this end, the output intensity-modulated clock pulse sequence $P_{o / p}$ after using Eq. (1) and Eq. (13) can be expressed as follows:

$$
P_{o / p}(m)=\frac{P_{p} \cdot(1+m \cdot \cos (\Omega \cdot k \cdot T))}{\left[1-\left(1-\left(1 / G_{0}\right)\right) \cdot \exp \left(-P_{p} \cdot(1+m \cdot \cos (\Omega \cdot k \cdot T)) \cdot A / U_{\text {sat }}\right)\right]}
$$

Eq. (14) depicts that $P_{o / p}$ is a function of the SOA gain $G_{0}$, the saturation energy $U_{\text {sat }}$ and the pulse peak power $P_{0} \cdot(1+m \cdot \cos (\Omega \cdot k \cdot T))$. By expanding (14) in a first-order Taylor series around $m=0$ the output pulse peak power can be written as the sum of a $d c$ signal component and an oscillation term $a c$ at $\Omega$ :

$$
P_{p / o}(m)=\left.P_{o / p}\right|_{m=0}+\left.\frac{\partial P_{o / p}}{\partial m}\right|_{m=0} \cdot m
$$


where the $d c$ component is expressed as shown in (16):

$$
\left.P_{o / p}\right|_{m=0}=\frac{P_{p}}{1-\left(1-\frac{1}{G_{0}}\right) \cdot \exp \left(-\frac{P_{p} \cdot A}{U_{s a t}}\right)}
$$

and the ac component as

$$
\left.\frac{\partial P_{o / p}}{\partial m}\right|_{m=0}=\left[\frac{P_{p}}{1-\left(1-\frac{1}{G_{0}}\right) \cdot \exp \left(-\frac{P_{p} \cdot A}{U_{s a t}}\right)}-\frac{P_{p} \cdot\left(1-\frac{1}{G_{0}}\right) \cdot \frac{P_{p} \cdot A}{U_{s a t}} \cdot \exp \left(-\frac{P_{p} \cdot A}{U_{s a t}}\right)}{\left[1-\left(1-\frac{1}{G_{0}}\right) \cdot \exp \left(-\frac{P_{p} \cdot A}{U_{s a t}}\right)\right]^{2}}\right] \cdot \cos (\Omega \cdot k \cdot T)
$$

Dividing all terms of Eq. (15) by the $d c$ component, we can calculate the modulation depth index at the output by dividing Eq. (17) by Eq. (16) and then multiplying by $m$, as shown in (18):

$$
m_{o / p}=[a c(\text { component }) / d c(\text { component })] \cdot m
$$

Finally, the modulation depth index of the output pulse peak power $m_{o / p}$ is found to be:

$$
m_{o / p}=\frac{1-\left(1-\frac{1}{G_{0}}\right) \cdot \exp \left(-\frac{P_{p} \cdot A}{U_{\text {sat }}}\right) \cdot\left(1+\frac{P_{p} \cdot A}{U_{\text {sat }}}\right)}{1-\left(1-\frac{1}{G_{0}}\right) \cdot \exp \left(-\frac{P_{p \cdot A}}{U_{\text {sat }}}\right)} \cdot m
$$

Eq. (19) provides a complete description of the SOA amplifier response to an injected intensitymodulated clock pulse sequence. It shows that the intensity modulation at the output is linearly related to the intensity modulation at the input and that the constant of proportionality depends on the SOA steady-state gain $G_{0}$, the average peak power $P_{\mathrm{p}}$ and the saturation energy $U_{\text {sat. }}$ In addition, the intensity modulation always decreases at the output of the amplifier. To relate the input and output intensity modulation indices $m$ and $m_{o / p}$ respectively, we define the Intensity Modulation Reduction (IMR) index as

$$
\operatorname{IMR}=10 \cdot \log \left|m_{o / p} / m\right|
$$


Given that the intensity modulation depth indices $m$ and $m_{\mathrm{o} / \mathrm{p}}$ of the input and the amplified output pulses are in principle the amplitudes of a slow varying frequency component at $\Omega$ inducing the power fluctuations on the pulses [20], Eq. (20) indicates the Intensity Modulation Reduction (IMR) of this frequency component after the SOA amplification process. In specific, the $m_{\mathrm{o} / \mathrm{p}}$ power level at the SOA's exit will be reduced from its respective power level at the input $m$ by an amount equal to the IMR value.

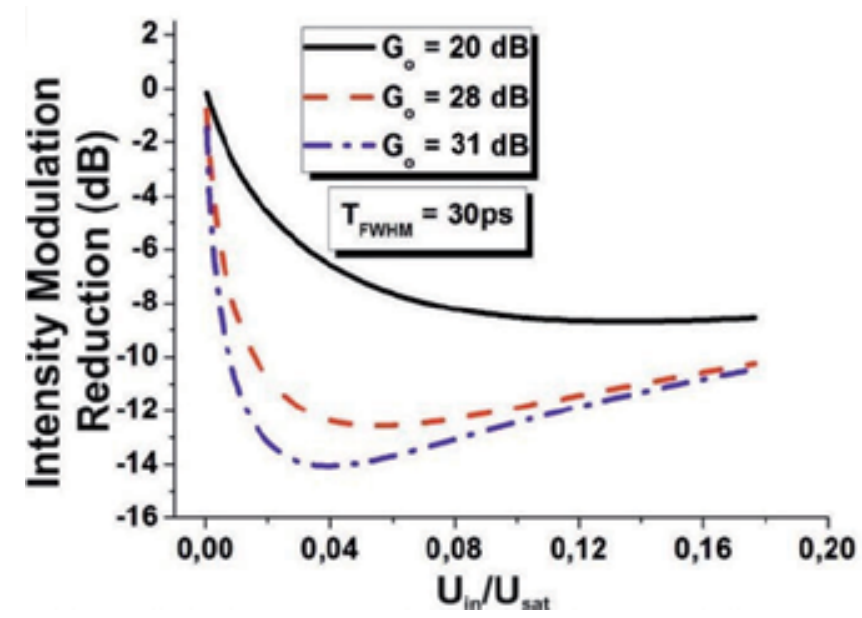

Figure 3. Theoretical Intensity Modulation Reduction (IMR) expressed in dB vs. $U_{\text {in }} / U_{\text {out }}$ values. IMR is illustrated for different SOA gain levels in the case of 30-ps-long pulses.

Figure 3 depicts the graphical representation of Eq. (20) for different SOA gains versus $U_{\text {in }} / U_{\text {sat }}$ quantity. The values of IMR represent a negative quantity since the output modulation depth is always smaller than the respective values at the input. A rapid drop of IMR leading to enhanced intensity modulation suppression is illustrated in Figure 3, when $U_{\text {in }} / U_{\text {sat }}$ is almost 0.04 and the pulsewidth is equal to 30 ps for all SOA gain levels. Figure 3 shows that when SOA operates in low gain level corresponding to a gain value of $20 \mathrm{~dB}$, a nearly constant IMR of $8 \mathrm{~dB}$ is obtained. However, for higher gains, the IMR increases to reach $14 \mathrm{~dB}$ for a SOA gain of $31 \mathrm{~dB}$. In addition, the maximum intensity modulation reduction for a SOA gain value equal to $31 \mathrm{~dB}$ is achieved when $U_{\text {in }} / U_{\text {sat }}$ quantity takes values between 0.01 and 0.05 , implying that the SOA is capable of suppressing a large power variation at its input. As $U_{\text {in }}$ increases, the IMR curves present a flat form and it is clamped to a constant level irrespective of the inserted pulse energy, after this specific $U_{\text {in }}$ threshold.

\section{Experiment and results}

The scope of this section is to provide experimental verification of the theoretical analysis for the deterministic timing jitter and the intensity modulation reduction induced by the SOA 
amplification process. Figure 4 demonstrates the experimental setup that was used for measurements with different pulsewidths and SOA gain levels. It consists of a $1549.2 \mathrm{~nm}$ modelocked laser (TMLL) and a Ti:LiNbO-3 electro-optic modulator (MOD) driven by a $10 \mathrm{~Gb} / \mathrm{s}$ pattern of alternating " 1 "s and " 0 "s, to create clock pulses at $5 \mathrm{GHz}$, so as to ensure a pulse period greater than the SOA gain recovery time (160 ps 1/e).

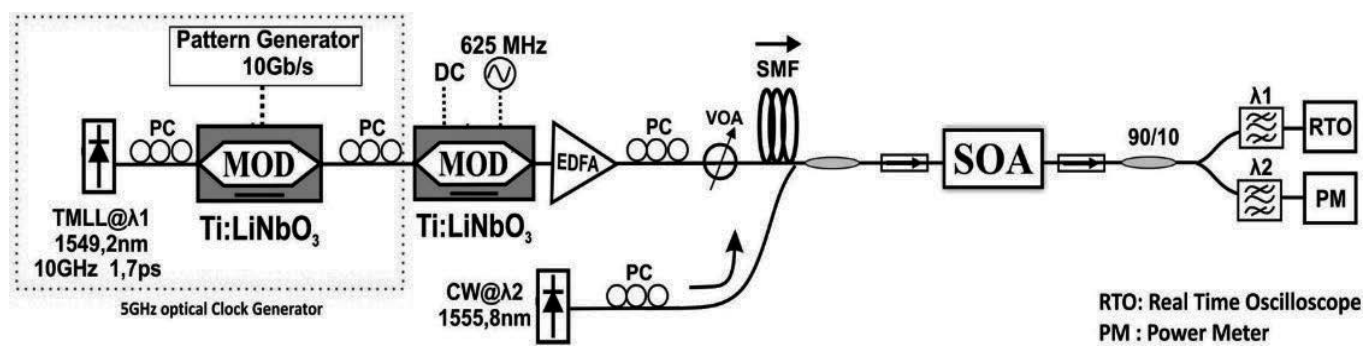

Figure 4. Experimental setup used for the deterministic timing jitter and intensity modulation reduction measurements.

In order to create an intensity-modulated pulse sequence, the clock signal is then injected into a second modulator driven by a $625 \mathrm{MHz}$ sinusoidal signal that creates pulses with 8 different pulse peak power levels. The intensity-modulated clock signal is amplified via an erbium doped fiber amplifier (EDFA) in order to compensate the losses and properly adjust the required power levels of optical pulses before their introduction into the SOA. Two fiber spools of $800 \mathrm{~m}$ and $1225 \mathrm{~m}$ were employed to enable pulsewidth adjustment at 20 ps and $30 \mathrm{ps}$ by exploiting the fiber dispersion. An additional CW beam at $1558.2 \mathrm{~nm}$ was utilized to adjust SOA gain level and as such to determine its operational regime. After setting the SOA gain to the desired value, the output pulse train was captured on a real-time oscilloscope with $16 \mathrm{GHz}$ bandwidth and a jitter measurement floor of $300 \mathrm{fs}$, where the jittery pulses were collected for offline postprocessing. The experimental setup of Figure 4 was also used in order to experimentally verify the IMR graphs shown in Figure 3. By varying the CW signal inserted into the SOA in order to cover a broad operational SOA gain regime, the intensity modulation of the input signal, defined as the highest to the lowest pulse peak power ratio, was measured at the output of the SOA. The operation of the amplifier both in the nonsaturated regime and in the saturated regime was also ensured by properly adjusting the input signal power. By calculating the difference between the initial and the output modulation depth values, the experimental data of IMR for every different SOA gain level was obtained. The control and input signals were adjusted in terms of power and polarization by means of variable optical attenuators (VOA) and polarization controllers (PC). The SOA module was a 1.5-mm-long multiquantum well structure with a small signal gain of $31 \mathrm{~dB}$. The device was driven at $450 \mathrm{~mA}$ and the $U_{\text {sat }}$ parameter of the SOA was found to be approximately $7 \mathrm{fJ}$. The jittery pulses that were captured on the real-time oscilloscope at $100 \mathrm{GSa} / \mathrm{s}$ for offline postprocessing. The collected optical pulses were reconstructed with a sample time resolution of $\Delta t=1.25 \mathrm{ps}$, after 8-fold upsampling. For each run, the total output timing jitter, referred to as $J^{\text {TOTAL }}$, was calculated over 
8192 pulses by means of Eq. (4) [15]. The total output timing jitter referred as $J^{\text {TOTAL }}$ consists of noise-induced random jitter and deterministic jitter [23]. The deterministic timing jitter stems from pulse edge variations depending on the input data characteristics. On that account, it is crucial to separate the stochastic contribution of random jitter from the deterministic process that is only responsible for timing variations proportional to the pulses' intensity modulation. Thus, deterministic jitter is calculated as a peak-to-peak value $\left(J_{\mathrm{pp}}^{D}\right)$ between a minimum and a maximum value since a probabilistic distribution cannot be applied. On the contrary, random jitter is determined as the root-mean-square (rms) value of a normal distribution $\left(J_{\mathrm{pp}}^{R}\right)$. By using well-known equations of converting the root-mean-square (rms) to peak-to-peak values $[24,25]$ the total timing jitter $J^{\text {TOTAL }}$ can be finally calculated as the following:

$$
J^{\text {TOTAL }}=J_{\mathrm{pp}}^{R}+J_{\mathrm{pp}}^{D}
$$

The total timing jitter at the output of the SOA in the absence of pulse peak power variations is uncorrelated to the timing jitter induced from an intensity-modulated pulse sequence [15]. As such, it represents the accumulated random timing jitter of our experimental system:

$$
J_{\mathrm{pp}}^{R}=\left.J^{\mathrm{TOTAL}}\right|_{\text {intensity modulation }=0 \mathrm{~dB}}
$$

Based on this assumption, the deterministic timing jitter induced by the SOA amplification process can be calculated by subtracting the random jitter measurement floor from $J$ TOTAL when the input pulse sequence has a given intensity modulation [15]. Table 1 summarizes the timing jitter values for power-equalized pulses when the pulsewidth values are $20 \mathrm{ps}$ and 30

\begin{tabular}{|c|c|c|c|c|c|}
\hline & \multicolumn{2}{|r|}{ SOA Input } & \multicolumn{3}{|c|}{ SOA Output } \\
\hline Pulsewidth (ps) & \multicolumn{2}{|r|}{ Random Jitter (ps) } & \multirow[t]{2}{*}{ Gain $(\mathrm{dB})$} & \multicolumn{2}{|c|}{ Random Jitter Floor $J_{\mathrm{PP}}^{R}(\mathrm{ps})$} \\
\hline Nonsaturation & $\begin{array}{c}\text { Saturat } \\
\text { on }\end{array}$ & & & & \\
\hline 20 & \multirow{2}{*}{$J_{\mathrm{rms}}^{R}$} & 0.585 & \multirow{2}{*}{20} & 4.356 & 4.391 \\
\hline 30 & & 0.634 & & 5.065 & 5.180 \\
\hline 20 & \multirow{2}{*}{$J_{\mathrm{PP}}^{R}$} & 4.048 & \multirow{2}{*}{31} & 6.082 & 4.223 \\
\hline 30 & & 4.460 & & 6.596 & 5.779 \\
\hline
\end{tabular}
ps and the SOA gains equal to $20 \mathrm{~dB}$ and $31 \mathrm{~dB}$, respectively.

Table 1. Timing jitter values for power-equalized pulses at the input and at the output of the SOA 


\subsection{Deterministic timing jitter: Theoretical and experimental results}

Figure 5(a) and 5(b) show the eyediagrams of a $10 \mathrm{~dB}$ intensity-modulated input signal with 20 ps and 30 ps pulsewidths, respectively. Figure 5(c) and 5(d) depict the output eyediagrams for the two pulsewidths when the amplifier operates in the nonsaturated regime. Figure 5(e) and 5(f) illustrate similar results for the two pulsewidths in case the SOA is operated in the strongly saturated gain region. The experimental average peak power values for the eyediagrams obtained in Figure 5 are shown in Table 2. In the eyediagrams of Figure $5(\mathrm{c}-\mathrm{f})$, the deterministic jitter is masked under the contribution of total jitter including the accumulated random jitter of the system as well. The irregular shapes of the output eye diagrams reveal, however, the pulse shape distortion that triggers the deterministic timing jitter.

\begin{tabular}{ccc}
\hline SOA Gain & & $\mathbf{2 0 ~ d B}$ \\
\hline Pulsewidth & Nonsaturated & Saturated \\
\hline $20 \mathrm{ps}$ & $30 \mu \mathrm{W}$ & $1.85 \mathrm{~mW}$ \\
\hline $30 \mathrm{ps}$ & $34 \mu \mathrm{W}$ & $1.23 \mathrm{~mW}$ \\
\hline
\end{tabular}

Table 2. Experimental values for the obtained SOA eyediagrams in Figure 5.

Figure $5(\mathrm{~g}-\mathrm{j})$ depicts the experimental and theoretical results of the deterministic timing jitter versus input signal intensity modulation expressed in $\mathrm{dB}$, for different gain levels, saturation regimes of the SOA and pulsewidths.
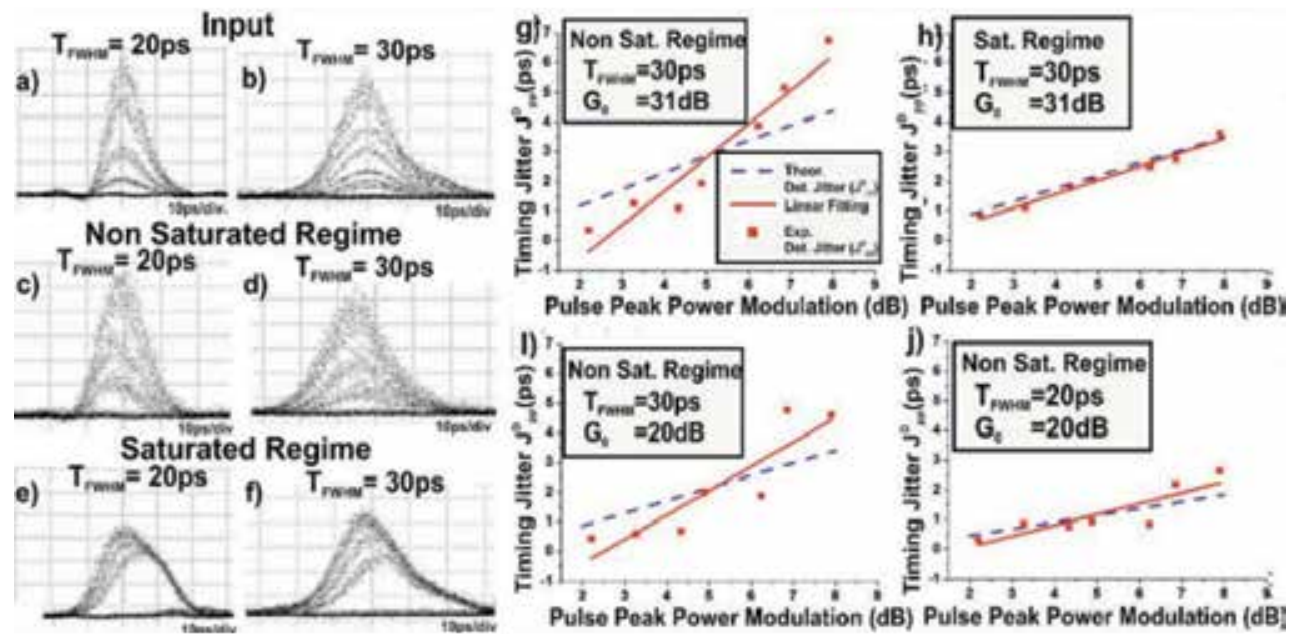

Figure 5. (a), (b) Eyediagram of an input optical signal with $10 \mathrm{~dB}$ intensity modulation with a pulsewidth of (a) 20 ps and (b) 30 ps, and the respective SOA output when the amplifier operates in the (c), (d) nonsaturated and (e),(f) strongly saturated regimes. The SOA gain level is equal to $20 \mathrm{~dB}$ for $(\mathrm{a}-\mathrm{f})$. Timescale for $(\mathrm{a}-\mathrm{f})$ : $10 \mathrm{ps} / \mathrm{div}$. Experimental and theoretical results for the deterministic timing jitter vs. pulse peak power modulation (intensity modulation) expressed in $\mathrm{dB}$, for 30 ps pulsewidth and $31 \mathrm{~dB}$ SOA gain in $(\mathrm{g})$ nonsaturated and (h) strongly saturated regimes, and for $20 \mathrm{~dB}$ SOA gain and nonsaturated region with (i) $30 \mathrm{ps}$ and (j) 20 ps pulsewidths, respectively. 
According to Eq. (11), the theoretical deterministic timing jitter depends linearly on intensity modulation levels. On that account, a linear fit was applied to the experimental data revealing good agreement between theoretical and experimental results obtained in all cases [15]. Figure $5(\mathrm{~g})$ and $(\mathrm{h})$ show theoretical deterministic timing jitter results obtained by applying Eq. (7) into Eq. (8), as well as the experimental data with their linear fit for 30 ps pulsewidth and 31 $\mathrm{dB}$ SOA gain level. The average pulse peak power values used in this case were $11 \mu \mathrm{W}$ and $235 \mu \mathrm{W}$ for unsaturated and saturated SOA operations, respectively. The graphs reveal a reduction of deterministic timing jitter in excess of $25 \%$ for the case of the SOA saturated operational regime. Figure 5(i) illustrates deterministic timing jitter evolution versus intensity modulation levels for $20 \mathrm{~dB}$ SOA gain using an average pulse peak power value of $34 \mu \mathrm{W}$. When compared with Figure 5(g), a decrease of the deterministic timing jitter values with the SOA gain level is evident. Finally, Figure 5(i) and 5(j) depict the deterministic timing jitter results for 30 ps and 20 ps pulsewidths, respectively, when all other operating parameters are the same, confirming that shorter pulses generate lower deterministic timing jitter levels [30 W average pulse peak power values for Fig. 5(j)]. In all cases, good qualitative and quantitative agreement between experiment and theory was achieved retaining the same deterministic timing jitter trend.

\subsection{Intensity modulation reduction: Theoretical and experimental results}

Figure 6 depicts the theoretical and experimental results for the output $m_{\mathrm{o} / \mathrm{p}}$ versus the input $m$ modulation depth indices and the $m_{\mathrm{o} / \mathrm{p}} / m$ versus the input pulse energy $U_{\text {in }}$ of optical pulses inserted in the SOA. The theoretical curves are denoted by solid lines, and the experimental observations by bullets. A linear fit was also applied to the experimental data in Figure 6(a), 6(b) and 6(d), due to the linear nature of the IMR indicated by Eq. (19) and it is represented by a dashed line. As can be noticed in Figure 6(a) and 6(b), the output modulation depth index depends linearly on the input modulation depth index when the SOA gain levels equal to 20 $\mathrm{dB}$ and $28 \mathrm{~dB}$, respectively. In both cases, the amplifier operates in the nonsaturated region with the $U_{\text {in }}$ values reaching up to $5 \mathrm{fJ}$ and $0.5 \mathrm{fJ}$, respectively. Figure 6(c) demonstrates the $m_{\mathrm{o} / \mathrm{p}} / m$ ratio versus the input pulse energy $U_{\mathrm{in}}$ for SOA gain levels of $20 \mathrm{~dB}, 28 \mathrm{~dB}$ and $31 \mathrm{~dB}$. As the gain level rises from $20 \mathrm{~dB}$ to $31 \mathrm{~dB}$, the steepness of the slope increases and the curve shifts closer to the axis. In the case of gain equal to $31 \mathrm{~dB}$, both unsaturated and saturated SOA experimental observations are shown in Figure 6(d) revealing higher intensity modulation reduction for strongly saturated SOA with the $U_{\text {in }}$ reaching $2 \mathrm{fJ}$. Figure $6(\mathrm{~d})$ presents in detail the output $m_{\mathrm{o} / \mathrm{p}}$ versus the input $m$ modulation indices for the two operational SOA regimes for $31 \mathrm{~dB}$ gain level. The $U_{\text {in }}$ value is directly associated to the SOA operational regime and imposes the slope of the $m_{\mathrm{o} / \mathrm{p}}$ versus $m$ curve resulting in low or high variation between input and output modulation depth that in turn yields low- or high-intensity modulation reduction values. It can be observed that the slope of the curve is smaller in the case of a saturated SOA corresponding to $U_{\text {in }}$ equal to $2 \mathrm{fJ}$ in comparison with the unsaturated SOA referring to a $U_{\text {in }}$ value equal to $0.2 \mathrm{fJ}$. 

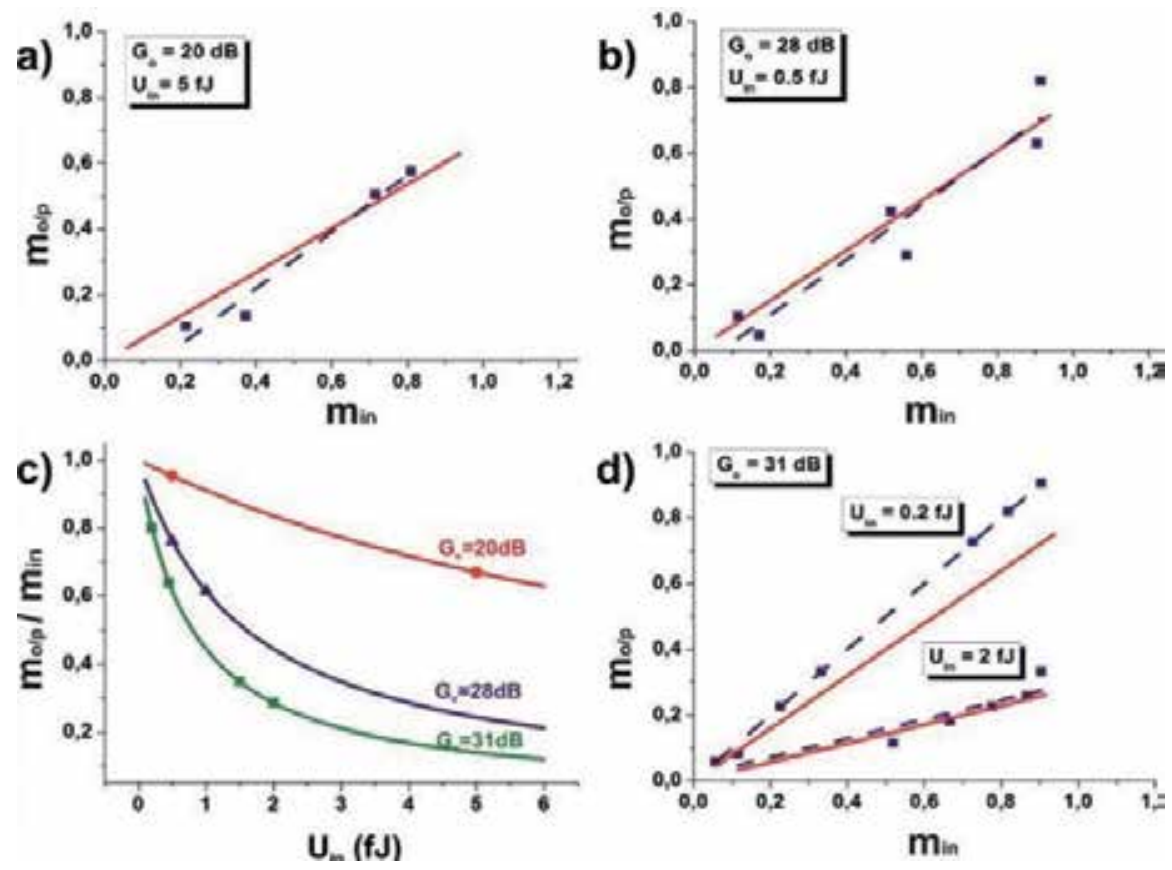

Figure 6. (a), (b) and (d) experimental and theoretical results for output modulation depth index versus input modulation depth index of intensity-modulated optical pulses for $\mathrm{G}_{0}=20 \mathrm{~dB}, \mathrm{G}_{0}=28 \mathrm{~dB}$ and $\mathrm{G}_{0}=31 \mathrm{~dB}$ of the SOA. (d) Results in both nonsaturation and saturation regime of the SOA and (c) intensity modulation reduction $\left(m_{\mathrm{o} / \mathrm{p}} / m_{\mathrm{in}}\right)$ for the three SOA gain levels vs. $U_{\text {in }}$. In all cases, the bullets represent experimental measurements, the solid lines the respective theoretical curves and the dashed lines the experimental fit.

Comparison between dashed and solid lines in Figure 6(a-d) shows good agreement between theory and experiment and indicates the SOA potential to provide increased pulse peak power equalization at its output, when operating the amplifier in the saturation regime.

\section{Discussion}

The theoretical framework and its experimental verification for both deterministic timing jitter and intensity modulation reduction analysis have relied on the assumption that every pulse experiences the same initial steady-state gain. This assumption allowed for the treatment of the pulse sequence on a per pulse basis and for the use of clock pulses for its experimental validation. However, the theoretical analysis presented here can also be extended towards calculating both these phenomena, in the case of random data patterns with intensitymodulated pulses used as the input signal in SOAs.

In the case of deterministic timing jitter, when the SOA gain recovery time is faster than the bit period, all the incoming data pulses will again experience the same steady-state gain $G_{0}$ inside the amplifier. This condition allows Eq. (7) of the mean arrival time $T_{\text {MEAN }}$ to be valid. But even if the SOA gain recovery time is slower than the bit period, Eq. (7) can be exploited 
for calculating the mean arrival time $T_{\text {MEAN }}$ of the data pulses and, subsequently, the deterministic timing jitter. In this case, the use of a random incoming data pattern into the SOA will actually result in different gain levels experienced by every individual optical pulse. On that account, $G_{0}$ should then be treated as an additional variable in Eq. (7) with its values residing within a certain range $\Delta G_{0}$. This actually turns relationship (7) into a two-variable function, assuming a given pulsewidth and a constant $U_{\text {sat }}$ parameter [15].

According to Figure 1, the same pulse peak power level results in a lower absolute value for the pulse mean arrival time when a lower gain value is perceived by the pulse. In Figure 1, for example, the absolute value of $T_{\text {MEAN }}$ for a gain of $20 \mathrm{~dB}$ is always lower than the respective value for a SOA gain of $28 \mathrm{~dB}$, which in turn is always lower than the respective value for a 30 $\mathrm{dB}$ SOA gain. This indicates that the deterministic jitter in case of different gain levels perceived by every pulse, as will be the case with random incoming data patterns and gain recovery times slower than the bit period, will be always slightly higher compared to the deterministic jitter values induced by the same pulse sequence when the SOA gain recovery time is faster than the bit period. For example, when the incoming data pulse with the lowest peak power level enters the amplifier after a long sequence of " 1 "s, it experiences the lowest SOA gain among all data pulses yielding, in this way, the lowest absolute value for its mean arrival time at the SOA output. At the same time, the highest data pulse comes after a long sequence of " 0 "s, so that it actually experiences the full gain of the amplifier, resulting in the highest absolute value among all data pulses for its mean arrival time $T_{\text {MEAN }}$. This scenario can certainly occur when a truly random data pattern with an intensity modulation that follows a certain statistical distribution will be injected into the SOA [15]. To this end, Eq. (7) indicates that higher deterministic timing jitter values should be expected in this case.

Following the same rationale, Eq. (19) of the modulation depth index of the output pulse peak power $m_{\mathrm{o} / \mathrm{p}}$ can also turn into a two-variable function $\left(G_{0}, P_{\mathrm{p}}\right)$ for a certain pulse waveform A and a constant $U_{\text {sat }}$ parameter. In this way, it enables its utilization in cases of random data pattern when the SOA gain recovery time is greater than the bit period. Again, the worst-case scenario in terms of the intensity modulation level of the output pulses will take place when the pulses with the smallest peak power level follow a long sequence of "1"s and the pulses with the highest peak power level come after a long sequence of "0"s. The smallest pulses will experience the lowest gain while the highest pulses will perceive the full gain of the amplifier resulting in a less optimal peak power equalization compared to the respective case where the SOA gain recovers, and all pulses receive the same steady-state gain $G_{0}$. In such a traffic scenario, the random data pattern will limit the power equalization dynamics of the SOA, since the bit randomness will affect its ability to provide the same gain to every pulse and will lead to varying gain values that will be imprinted on the amplified optical pulses.

\section{Conclusion}

Research interest in semiconductor optical amplifiers (SOAs) has been lately renewed since SOAs appear as the most preferable on-chip amplifier option in many key network subsystems. 
Although a concerted research effort on SOA-based devices spanning the last 20 years, has revealed most of their underlying amplification secrets, SOA effects on intensity-modulated optical pulses in terms of timing jitter and pulse peak power equalization have not yet been consolidated in a detailed analytical framework. On that account, we have presented in this chapter, a holistic theoretical framework verified by experimental results that establishes for the first time a systematic methodology for the deterministic timing jitter and peak power equalization estimation in case of intensity-modulated optical pulses entering the SOA. Experimental and theoretical results reveal a linear relationship between deterministic timing jitter and intensity modulation levels when the SOA gain recovery time is shorter than the bit period. The pulse mean arrival time is calculated as a function of the pulse peak power, the pulsewidth and the SOA steady-state gain. In addition, pulse peak power equalization analysis shows that intensity modulation at output is linearly related to the intensity modulation at the input and the constant of proportionality depends on the SOA steady-state gain $G_{0}$, the pulse peak power $P_{\mathrm{p}}$ and the saturation energy $U_{\text {sat }}$. Both deterministic timing jitter and intensity modulation reduction formulas derived in the proposed theoretical analysis, enable a qualitative and quantitative insight into the SOA performance when intensity-modulated optical pulses are inserted into the amplifier.

\section{Acknowledgements}

This work has been supported in part by the European Commission through FP7-ICT-IP project PhoxTrot (contract no. 318240) and FP7 MC-IAPP project COMANDER (contract no. 612257).

\section{Author details}

T. Alexoudi ${ }^{1,2 *}$, G.T. Kanellos², S. Dris ${ }^{3}$, D. Kalavrouziotis ${ }^{3}$, P. Bakopoulos ${ }^{3}$, A. Miliou ${ }^{1,2}$ and N. Pleros ${ }^{1,2}$

*Address all correspondence to: theonial@iti.gr

1 Information \& Technologies Institute, Center for Research \& Technology Hellas, Thessaloniki, Greece

2 Department of Informatics, Aristotle University of Thessaloniki, Thessaloniki, Greece

3 School of Electrical Engineering and Computer Engineering, National Technical University of Athens, Athens, Greece 


\section{References}

[1] W. Freude et al. Linear and nonlinear semiconductor optical amplifiers. In: Proceedings of 12th International Conference on Transparent Optical Networks (ICTON 2010); 27 June-1 July; Munich, Germany. IEEE; 2010. p. 1-4. DOI: 10.1109/ICTON. 2010.5549097

[2] Dong, X. Zhang, S. Fu, J. Xu, P. Shum and D. Huang. Ultrafast all-optical signal processing based on single semiconductor optical amplifier. Journal of Selected Topics in Quantum Electronics. 2008; 14(3):770-778. DOI: 10.1109/JSTQE.2008.916248

[3] J. Leuthold. All-optical wavelength conversion up to $100 \mathrm{Gbit} / \mathrm{s}$ with SOA delayedinterference configuration. OSA Trends in Optics and Photonics. 2000; 44(Optical Amplifiers and Their Applications):OWB3.

[4] Z. Li et al. All-optical logic gates using semiconductor optical amplifier assisted by optical filter. Electronic Letters. 2005; 41(25):1397-1399. DOI: 10.1049/el:20053385

[5] G. Berrettinni, A. Simi, A. Malacarne, A. Bogoni, and L. Potí. Ultrafast integrable and reconfigurable XNOR, AND, NOR and NOT photonic logic gate. IEEE Photonics Technology Letters. 2006; 18(8):917-919. DOI: 10.1109/LPT.2006.873570

[6] A. Hamie, A. Sharaiha, M. Guégan, and B. Puce. All-optical logic NOR gate using two-cascaded semiconductor optical amplifiers. IEEE Photonics Technology Letters. 2002;14(10):1439-1441. DOI: 10.1109/LPT.2002.802426

[7] G. T. Kanellos et al. All-optical 3R burst mode reception at $40 \mathrm{~Gb} / \mathrm{s}$ using 4 integrated MZI switches. IEEE/OSA Journal of Lightwave Technology. 2007; 25(1):184-192. DOI: 10.1109/JLT.2006.888169

[8] L. Aggazi et al. Monolithic integration of the erbium-doped amplifier with siliconon-insulator waveguides. OSA Optics Express. 2010;18(26):27703-27711. DOI: 10.1364/OE.18.027703

[9] C.S. Nicholes et al. An 8x8 InP monolithic tunable optical router (MOTOR) packet forwarding chip. IEEE/OSA Journal of Lightwave Technology. 2010;28(4):641-650. DOI: $10.1109 /$ JLT.2009.2030145

[10] V.S. Pato et al. All-optical burst mode power equalizer based on cascaded SOAs for 10-Gb/s EPONs. IEEE Photonics Technology Letters. 2008;20(24):2078-2080. DOI: 10.1109/LPT.2008.2006629

[11] D. Chiaroni et al. Optical packet ring network offering bit rate and modulation formats transparency. In: Proceedings of Optical Fiber Communication (OFC) Conference; 21-25 March; San Diego, CA, USA. IEEE; 2010. p. 1-3. DOI: 10.1364/OFC. 2010.OWI3 
[12] M. Settembre et al. Cascaded optical communication systems with in-line semiconductor optical amplifiers. IEEE/OSA Journal of Lightwave Technology. 1997; 15(6): 962-967. DOI: $10.1109 / 50.588666$

[13] G.P. Agrawal and N.A. Olsson. Self-phase modulation and spectral broadening of optical pulses in semiconductor laser amplifiers. IEEE Journal of Quantum Electronics. 1989; 25(11):2297-2306. DOI: 10.1109/3.42059

[14] T. Alexoudi, S. Dris, D. Kalavrouziotis, P. Bakopoulos, A. Miliou and N. Pleros. Timing jitter of SOA-amplified intensity modulated optical pulses. In: Optical Fiber Communication Conference (OFC); 4-8 March; Los Angeles, CA, USA. IEEE; 2012. p. 1-3. DOI: 10.1364/NFOEC.2012.JTh2A.13

[15] T. Alexoudi, G.T. Kanellos, S. Dris, D. Kalavrouziotis, P. Bakopoulos, A. Miliou and N. Pleros. Deterministic timing jitter analysis of SOA-amplified intensity modulated optical pulses. IEEE Photonics Journal. 2012; 4(5):1947-1955. DOI: 10.1109/JPHOT. 2012.2220341

[16] R. G.-Castrejón and A. Filios. Pattern-effect reduction using a cross-gain modulated holding beam in semiconductor optical in-line amplifier. IEEE/OSA Journal of Lightwave Technology. 2006; 24(12):4912-4917. DOI: 10.1109/JLT.2006.884972

[17] S. Bischoff, M.L. Nielsen and J J. Mork. Improving the all-optical response of SOAs using a modulated holding signal. IEEE/OSA Journal of Lightwave Technology. 2004; 22(5):1303-1308. DOI: 10.1109/JLT.2004.825354

[18] G. T. Kanellos, N. Pleros, C. Bintjas, H. Avramopoulos. SOA-based interferometric optical hard-limiter. In: Proceedings of Optical Amplifiers and their Applications (OAA) Conference; 30 June; San Francisco, CA, USA. OSA; 2004. DOI: 10.1364/IPR. 2004.JWB8

[19] K. Vlachos, G. Theophilopoulos, A. Hatziefremidis and H. Avramopoulos. 30 Gbps all-optical, clock recovery circuit. IEEE Photonics Technology Letters. 2000;12(6):705707. DOI: 10.1109/LPT.2002.801095.

[20] N. Pleros et al. Recipe for intensity modulation reduction in SOA-based interferometric switches. IEEE/OSA Journal of Lightwave Technology. 2004;22(12):2834-2841. DOI: 10.1109/JLT.2004.834834

[21] S.V. Kartalopoulos et al. Optical bit error rate: an estimation methodology. 1st ed. Willey-IEEE Press; 2004. 291 p. ISBN: 978-0-471-61545-3

[22] J. Peatross, S.A. Glasgow and M. Ware. Average energy flow of optical pulses in dispersive media. APS Physics Review Letters. 2000;84(11):2370-2373. DOI: http:// dx.doi.org/10.1103/PhysRevLett.84.2370

[23] R. Stephens. Analyzing jitter at high data rates. IEEE Communications Magazine. 2004; 42(2):S6-10. DOI: 10.1109/MCOM.2003.1267095 
[24] Agilent Technologies. Measuring jitter in digital systems, application note 1448-1 [Internet]. 1 June 2003. Available from http://www.colbyinstruments.com/pdfs/ 5988-9109EN.pdf [Accessed: 31-5-2015]

[25] Maxim Integrated. Converting between RMS to peak-to-peak Jitter at a specified BER, application note 462 HFAN-04.0.2 [Internet]. 2008 [Updated: 04/2008]. Available from: http://pdfserv.maximintegrated.com/en/an/AN462.pdf [Accessed: 31-5-2015] 
Chapter 6

\title{
Investigation of Broadband S-Band to L-Band Erbium- Doped Fiber Amplifier (EDFA) Module
}

\author{
Chien-Hung Yeh \\ Additional information is available at the end of the chapter \\ http://dx.doi.org/10.5772/61735
}

\begin{abstract}
This chapter presents three sections that describe the broadband S-band to L-band erbium-doped fiber amplifier modules. In the first section, an S-band gain-clamped erbiumdoped fiber amplifier (EDFA) module, employing a fiber Bragg grating (FBG) to act as a reflected element for generating a saturated tone injected into the EDFA module by using forward optical feedback method, is proposed. Moreover, the output performance of the gain and noise figure (NF) in the proposed gain-clamped S-band EDFA has been discussed in the wavelength range of $1478-1520 \mathrm{~nm}$. In the second section, we demonstrate experimentally a gain-flattened two-stage erbium-based fiber amplifier (EBFA) module, which is composed of by an erbium-doped waveguide amplifier (EDWA) and a C-band EDFA in serial structure. In an operation range of $1528-1562 \mathrm{~nm}$, the entire gain is larger than $35 \mathrm{~dB}$ and the observed NF is between 5.5 and $6.7 \mathrm{~dB}$. Moreover, $\pm 1.1 \mathrm{~dB}$ maximum gain variation is also obtained for the input signal power of $-25 \mathrm{dBm}$. Hence, the proposed fiber amplifier not only enhances the gain but also achieves the flatness in the wavelength region. In the final section, a broadband hybrid two-stage fiber amplifier, which is composed by a C-band EDFA and a C-band semiconductor optical amplifier $(\mathrm{SOA})$ in serial scheme, is investigated experimentally. Here, we only use a $3 \mathrm{~m}$ long erbium-doped fiber (EDF) serving as a preamplifier to increase the gain and reduce the noise figure. Therefore, the proposed hybrid amplifier achieves a $110 \mathrm{~nm}$ effectively amplification of 1500-1610 nm (from S- to L-band). In addition, the output performance of gain and NF in the proposed fiber amplifier has also been discussed.
\end{abstract}

Keywords: Erbium-Doped Fiber Amplifier (EDFA), Semiconductor Optical Amplifier (SOA), Erbium-Doped Waveguide, Amplifier (EDWA)

\section{Introduction}

Broadband erbium-doped fiber amplifier (EDFA) was useful to increase the number of wavelength-division-multiplexing (WDM) signals in $1.5-\mu \mathrm{m}$ fiber transmission, routing 
network, and optical access network [1]. These days, the EDFA module with $120 \mathrm{~nm}$ wavelength bandwidth can be accomplished theoretically and experimentally [2,3]. However, using the reconfigurable optical add-drop multiplexers (OADM) or carrying burst traffic in optical domain in the dynamic WDM systems, the power transient would be induced by the slow response of EDFA. This is a challenging issue to resolve. The transient effects could be reduced by utilizing either electric or optical control, or the combination of both techniques. In these schemes, the optical gain clamping was a commonly used technique. In the related researches, there have been some studies executed in the C- to L-band fiber amplifiers with gain-clamped effect [4-8]. In previous studies, serial architectures were employed [4, 5], predictably introducing cross talk among the C- to L-band ranges. Using parallel architecture could improve the cross talk [8], but the counter-propagating of transmitted signal and laser lightwave would produce the higher noise figure (NF) [9, 10]. Here, an S-band (1460-1520 nm) EDFA, which utilizes depressed cladding design and $980 \mathrm{~nm}$ pump laser to cause EDF gain extension effect, has been proposed [11]. Hence, the gain clamping technique is expected to extend to S-band by the proposed S-band EDFA.

In addition, a gain-flattened was also important key for EDFA dynamically working on WDM communication systems. Mostly, the gain spectra of EDFAs could be flattened by utilizing several methods, such as doping the different material compositions in the erbium-doped fiber (EDF) [12], or using optical filters to compensate for the variations in the gain spectra [13-19]. Moreover, different types of optical filters have been demonstrated for this issue, such as MachZehnder (M-Z) filters [18], fiber Bragg gratings (FBGs) [15], long-period fiber gratings [13, 14], fiber acousto-optic tunable filters [16, 17], a split-beam Fourier filter [19], and hybrid amplifier design [20].

Recently, to satisfy the requirement of communications capacity, the broadband EDFAs have been suiting the major techniques in the dense wavelength-division-multiplexed (DWDM) transmissions. Applying the C- to L-bands' gain of EDFA could be above $80 \mathrm{~nm}$ amplification bandwidth. And it would also enhance the ability of the transmission of DWDM systems [21]. Thus, there were many optimized architectures on C- plus L-band EDFAs [21-27]. And the broadband EDFA module design has the serial or parallel configuration individually [28, 29]. In L-band EDFA, the power conversion efficiency (PCE) was too low for obtaining the higher gain, because it was far from the erbium ion absorption band. In addition, several techniques to expand the L-band gain were demonstrated, such as utilizing unwanted C-band amplified spontaneous emission (ASE) [23], employing the double-pass configuration [30], [31], and applying reflection-type EDFA with grating [32].

In this chapter, there are three sections that describe the broadband S-band to L-band erbiumdoped fiber amplifier modules. In the first section, an S-band gain-clamped erbium-doped fiber amplifier (EDFA) module, employing a fiber Bragg grating (FBG) to act as a reflected element for generating a saturated tone injected into the EDFA module by using forward optical feedback method, is proposed. Moreover, the output performance of the gain and noise figure (NF) in the proposed gain-clamped S-band EDFA has been discussed in the wavelength range of 1478-1520 $\mathrm{nm}$. In the second section, we demonstrate experimentally a gain-flattened two-stage erbium-based fiber amplifier (EBFA) module, which is structured by an erbium- 
doped waveguide amplifier (EDWA) and a C-band EDFA in serial structure. In an operation range of 1528-1562 nm, the entire gain is larger than $35 \mathrm{~dB}$ and the observed NF is between 5.5 and $6.7 \mathrm{~dB}$. Moreover, $\pm 1.1 \mathrm{~dB}$ maximum gain variation is also obtained for the input signal power of $-25 \mathrm{dBm}$. Hence, the proposed fiber amplifier not only enhances the gain but also achieves the flatness in the wavelength region. In the final section, a broadband hybrid twostage fiber amplifier, which is constructed by a C-band EDFA and a C-band semiconductor optical amplifier (SOA) in serial scheme, is investigated experimentally. Here, we only use a $3 \mathrm{~m}$ long erbium-doped fiber (EDF) serving as a preamplifier to increase the gain and reduce the noise figure. Therefore, the proposed hybrid amplifier achieves a $110 \mathrm{~nm}$ effectively amplification of 1500-1610 nm (from S- to L-band). In addition, the output performance of gain and NF in the proposed fiber amplifier has also been discussed.

\section{S-Band gain-clamped EDFA module}

In this section, an S-band gain-clamped EDFA module, employing a fiber Bragg grating (FBG) to act as a reflected element for generating a saturated tone injected into the EDFA module by applying forward optical feedback method, is proposed experimentally. In the measurement, using a lasing wavelength could regularize the total population inversion under a homogeneously broadened effect for gain-clamped. Thus, the obtained gain of EDFA dependents on its absorption, emission cross sections, and the overlapping factor. Any variation of input signal power could be compensated by adjusting the power of lasing wavelength. As a result, each signal would experience a fixed gain through the EDFA module, with the variation of input signals power, which was caused by operation, such as signal adding or dropping. Figure 1 presents the proposed gain-clamped S-band EDFA module with forward optical feedback structure. The proposed fiber amplifier is constructed by an S-band EDFA, an optical circulator (OC), a $1 \times 2$ optical coupler $(\mathrm{CP})$, and three FBGs with different Bragg wavelengths. The optical $\mathrm{CP}$ has the input coupling ratios of $90,80,70$, and $50 \%$ for the input signal in Fig. 1, respectively. The optical CP with different input coupling ratio governs the forward injected power level of each saturated tone individually. The FBG is used to serve as a reflected element to generate a lasing wavelength in the S-band wavelength range. The lasing wavelength could be injected in forward direction for clamping the gain spectrum of proposed amplifier. Here, we utilize three different FBGs with various Bragg wavelengths and reflectivities and four optical CPs having different coupling ratios to produce the various injected wavelengths and powers for measuring the gain performance of proposed gain-clamped S-band EDFA module.

To support the sharp, high-attenuation, long-wavelength cutoff filter in active fiber, the S-band EDF with depressed-cladding design is proposed and used inside EDFA module [33]. The used EDF of first and second stages have various physical characteristics. In the first EDF stage, a $20 \mathrm{~m}$ long EDF is employed to provide low NF and medium gain by forward pumping. In second fiber stage, a $30 \mathrm{~m}$ long EDF is utilized to generate a large output power by backward pumping. Furthermore, the optical ISO between the two EDF stages is used to decrease the backward amplified spontaneous emission (ASE) noise and improve noise figure (NF). Besides, the total pumping power of $980 \mathrm{~nm}$ Laser Diode (LD) in the S-band EDFA module 


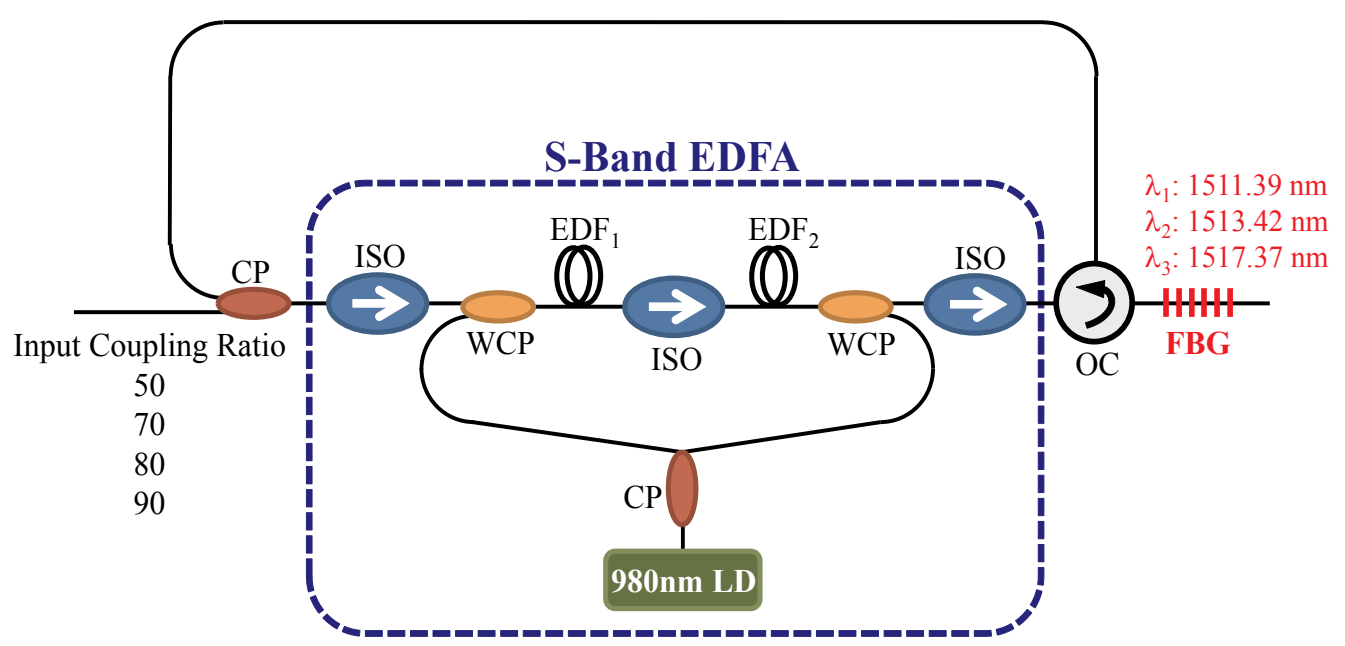

Figure 1. Experimental setup of gain-clamped S-band EDFA module by using forward optical feedback method.

can be set at $280 \mathrm{~mW}$, when the corresponding bias current is $356 \mathrm{~mA}$. To measure the performance of proposed gain-clamping EDFA, a tunable laser source (TLS) is employed to measure the gain and NF profiles. Moreover, the optical signal is observed by an optical spectrum analyzer (OSA) with a $0.05 \mathrm{~nm}$ resolution.

Figure 2 presents the gain and NF spectra of an S-band EDFA without gain-clamping over the operation region of $1478-1520 \mathrm{~nm}$, when the input signal power $(\mathrm{P})$ is $0,-20$, and $-40 \mathrm{dBm}$, respectively. As seen in Fig. 2, the gain and NF of 27.6 and $5.9 \mathrm{~dB}$ can be obtained at the wavelength of $1504 \mathrm{~nm}$ with the input power of $-20 \mathrm{dBm}$. And the saturated output power of $14.5 \mathrm{dBm}$ is also completed at $1498 \mathrm{~nm}$ under a $0 \mathrm{dBm}$ input power. The NFs are measured between 5.3 and $7.5 \mathrm{~dB}$ as the input signal power is $-20 \mathrm{dBm}$ in the wavelengths of $1478-1512$ $\mathrm{nm}$. Besides, the observed gain and NF are 12.4 and $9.6 \mathrm{~dB}, 26.4$ and $6.8 \mathrm{~dB}$, and 35.3 and 6.5 $\mathrm{dB}$, respectively, as seen in Fig 2, when the input signal powers are $0,-20$, and $-40 \mathrm{dBm}$ at the wavelength of $1510 \mathrm{~nm}$. Here, because some optical passive devices are placed at the input and output ends. Thus, the higher losses in C-band and the splice point of EDF and WDM coupler would produce the higher loss. So, the NF of this S-band EDFA module would be also degraded slightly.

To obtain the gain-clamped operation in S-band EDFA, the FBG is utilized in the proposed amplifier module for producing a saturated tone to fix the total population inversion, as shown in Fig 1. In this experiment, we employ three FBGs with different Bragg wavelengths and reflectivities successively for gain-clamping. Figure 3 shows the reflective spectrum of $\mathrm{FBG}_{1}$ $\left(\lambda_{1}\right)$ to $\mathrm{FBG}_{3}\left(\lambda_{3}\right)$ individually with the reflected Bragg wavelength of $1511.39 \mathrm{~nm}, 1513.42 \mathrm{~nm}$, and $1517.37 \mathrm{~nm}$, respectively. Here, the reflectivities of $\mathrm{FBG}_{1}, \mathrm{FBG}_{2}$, and $\mathrm{FBG}_{3}$ are measured at $91.83 \%, 93.11 \%$, and $82.98 \%$, respectively. Besides, the maximum wavelength shifts of three utilized S-band FBGs are around 2.0-2.3 nm while the strain is applied on the FBGs. Moreover, 


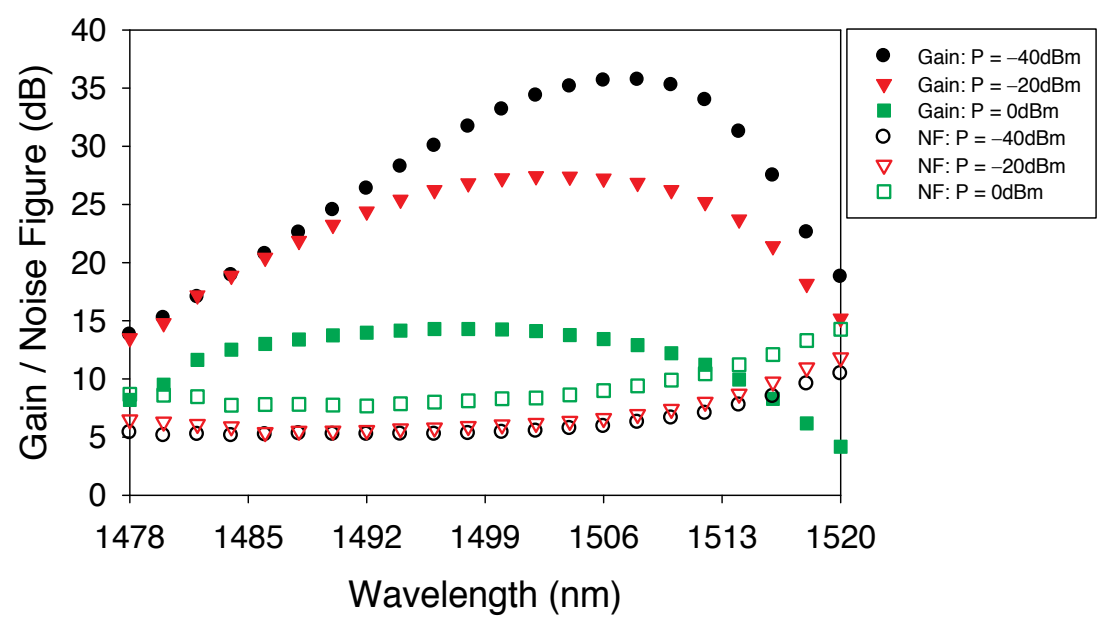

Figure 2. Measured gain and NF spectra of the original S-band EDFA over the wavelength region of 1478-1520 nm, while the input signal power $P$ is equal to $0,-20$, and $-40 \mathrm{dBm}$, respectively.

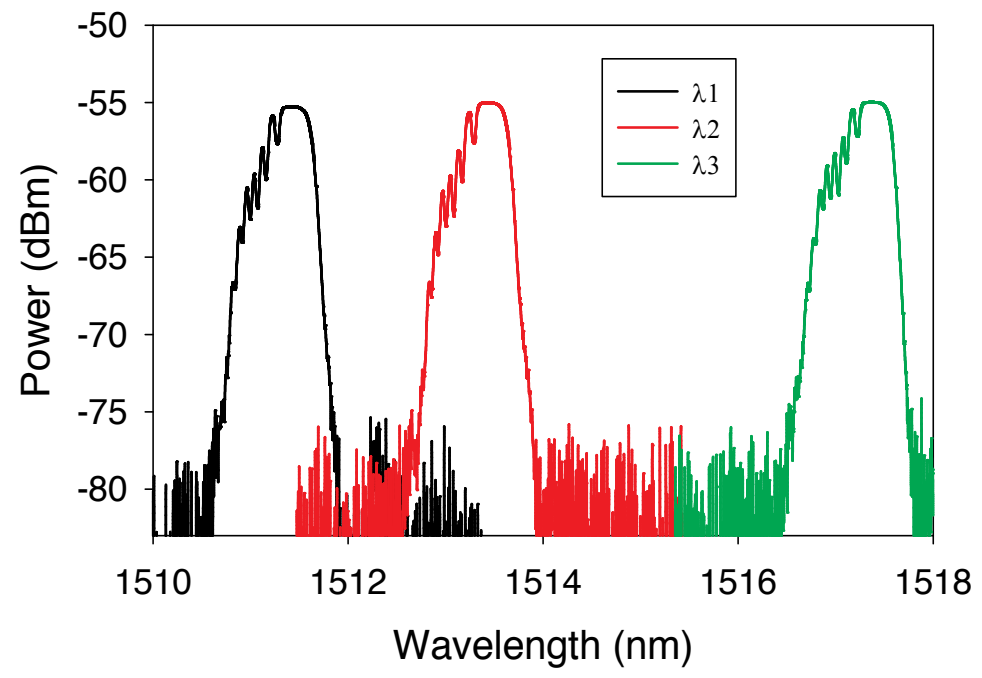

Figure 3. Measured reflective spectrum of FBG1-FBG3 with the central wavelength of $1511.39 \mathrm{~nm}(\lambda 1), 1513.42 \mathrm{~nm}$ $(\lambda 2)$, and $1517.37 \mathrm{~nm}(\lambda 3)$, respectively.

when the temperature is up to $\sim 90^{\circ} \mathrm{C}$, the reflected Bragg wavelength of FBG would drift nearly $1.2 \mathrm{~nm}$.

Figure 4 shows the gain and NF spectra under different powers in the input signal of $1506 \mathrm{~nm}$ without gain-clamping and with the various input coupling ratios of $90 \%, 80 \%, 70 \%, 50 \%$ for 
the input signal, when the saturated tones are set at (a) $1511.39 \mathrm{~nm}$, (b) $1513.42 \mathrm{~nm}$, and (c) 1517.37 nm, respectively. Figure 4(a) presents the gain and NF spectra under the different input signal powers. Here, Fig. 4(a) also displays the poorer gain and NF profiles at various coupling ratios of CP. Because of the higher gain region at 1508-1512 nm (as seen in Fig. 2), the saturated signal would obtain a largest gain for clamping and fixing the gain spectrum in the region and lead to the decrease of NF simultaneously. That is to say, while a saturated signal of 1511.39 $\mathrm{nm}$ backward injects into the EDFA module, it could get a most $\mathrm{Er}^{3+}$ ion population inversion to clamp the gain profile. It also would introduce the lower gain and poorer NF spectra in other input signals. Figure 4(b) presents that the gain could be maintained constant in the $-10 \mathrm{dBm}$ input signal power at an input coupling ratio of $90 \%$. Moreover, the constant gains are fixed at around $16.1 \mathrm{~dB}$ and the NF would be at $8.3-8.8 \mathrm{~dB}$, as the input signal power is less than $10 \mathrm{dBm}$. Figure $4(\mathrm{~b})$ shows the noise figure of $>10 \mathrm{~dB}$ in the input coupling ratio of $>80 \%$. At other operating conductions, the gain and NF profiles of Fig. 4(b) also are worse. However, these results are better than that of Fig. 4(a).

When a saturated tone is set at $1517.37 \mathrm{~nm}$ and an input coupling ratio is $50 \%$, the gain could be fixed constant in the input power of $<-12 \mathrm{dBm}$ under expense of around $6 \mathrm{~dB}$ gain. Then, the gain will be kept at around $19 \mathrm{~dB}$ and the NF could be measured between 8.2 and $9.6 \mathrm{~dB}$, as illustrated in Fig. 4(c). Here, the NF degradation is measured in $\sim 4.1 \mathrm{~dB}$. While the input coupling ratio is selected from 50 to $80 \%$, the gain is maintained at the input signal power of $<-12 \mathrm{dBm}$, as illustrated in Fig. 4(c). Besides, Fig. 4(c) also presents the gain dynamic range of $28 \mathrm{~dB}$ from -12 to $-40 \mathrm{dBm}$ when the input coupling ratio is $80 \%$. According to the results, if another channel is added or dropped into the proposed S-band EDFA, it does not influence the gain profile in the channel power of $<-12 \mathrm{dBm}$. In addition, as the gain-clamping is maintained, the input signal power should be putted in the input dynamic range, while the other input signal is added or dropped simultaneously. If not, the proposed EDFA does not bring the gain-clamping action. Figure 4(c) also shows that the gain is fixed at $25.3 \mathrm{~dB}$ in the input power of $<-15 \mathrm{dBm}$ while an input coupling ratio is set at $90 \%$. Therefore, the minimum NFs of $1 \mathrm{~dB}$ damage are measured, as seen in Fig. 4(c). Comparing Figs. 4(a) through 4(c), Fig. 4(c) has a better gain clamping result than that of Fig. 4(a) and Fig. 4(b). Thus, when the saturated wavelength is $>1515.69 \mathrm{~nm}$, it will obtain the larger clamped gain value and better $\mathrm{NF}$ value. If a saturated signal is disconnected from the larger gain range, it also will obtain a better gain and NF profiles, as seen in Fig. 4 (c). Therefore, the saturated tone at $1517.37 \mathrm{~nm}$ is a better choice for the proposed S-band gain clamped EDFA.

To investigate the gain-clamping performance, the gain and NF spectra are measured in the wavelength range of 1478-1520 $\mathrm{nm}$ at the effective input dynamic range according to the result of Fig. 4 (c). Thus, Fig. 5 presents the gain and NF spectra for the $S$ band gain-clamped EDFA in the wavelength range of $1478-1520 \mathrm{~nm}$ under the input ratio of $80 \%$ when the input signal power is $0,-15$, and $-40 \mathrm{dBm}$, respectively, and the saturated tone is selected at $1517.37 \mathrm{~nm}$. 

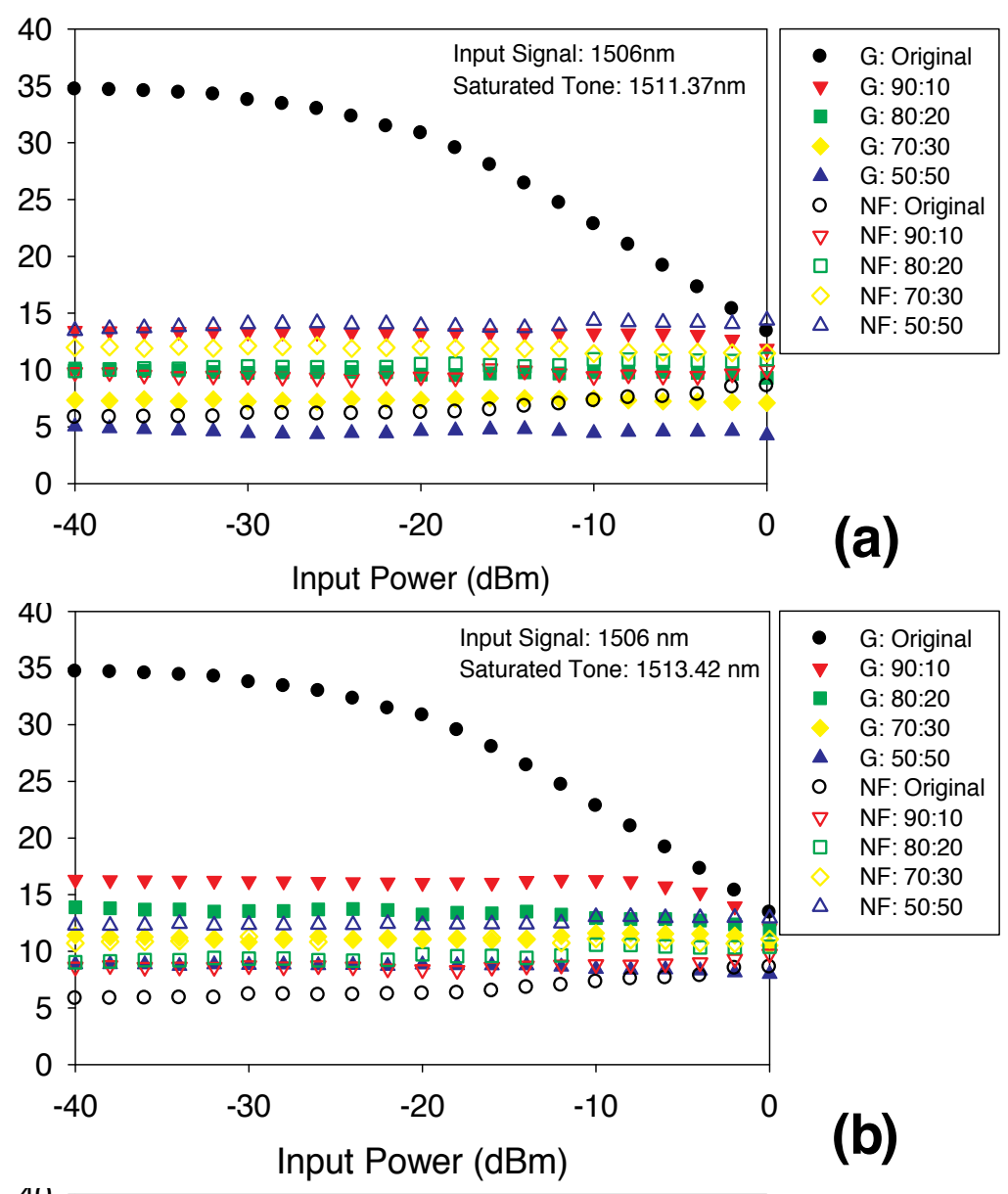

(b)

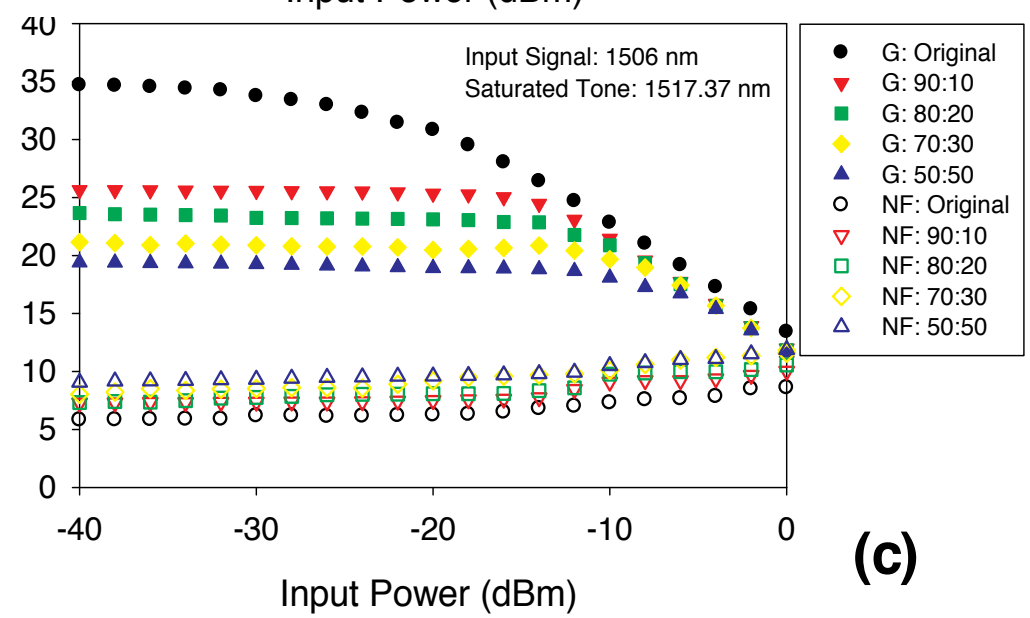

Figure 4. Measured gain and NF spectra without and with the input coupling ratio of $90,80,70,50 \%$ for the proposed gain-clamped EDFA under the saturated signals of (a) $1511.37 \mathrm{~nm}$, (b) $1513.42 \mathrm{~nm}$, and (c) $1517.37 \mathrm{~nm}$, respectively. 


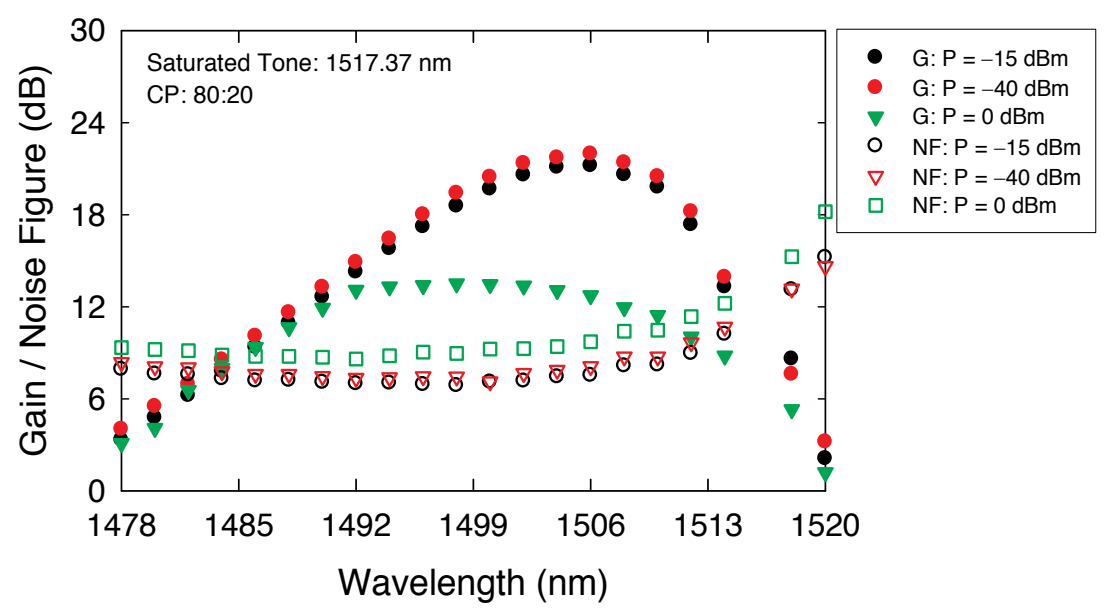

Figure 5. Measured gain and NF spectra of the S band gain-clamped EDFA in the wavelength of 1478-1520 nm with $80 \%$ input ratio at the input signal power $\mathrm{P}=0,-15$, and $-40 \mathrm{dBm}$ at the lasing wavelength of $1517.37 \mathrm{~nm}$, respectively.

In the measurement, the maximum gain of $24.7 \mathrm{~dB}$ is measured at the wavelength of $1502 \mathrm{~nm}$ for the input power of $-40 \mathrm{dBm}$. And the maximum gain variations are smaller than $0.6 \mathrm{~dB}$ in the operation range. As a result, the input gain dynamic range of $25 \mathrm{~dB}$ from -40 to $-15 \mathrm{dBm}$ could be detected and obtained, as shown in Fig. 5.

\section{D Graph 1}

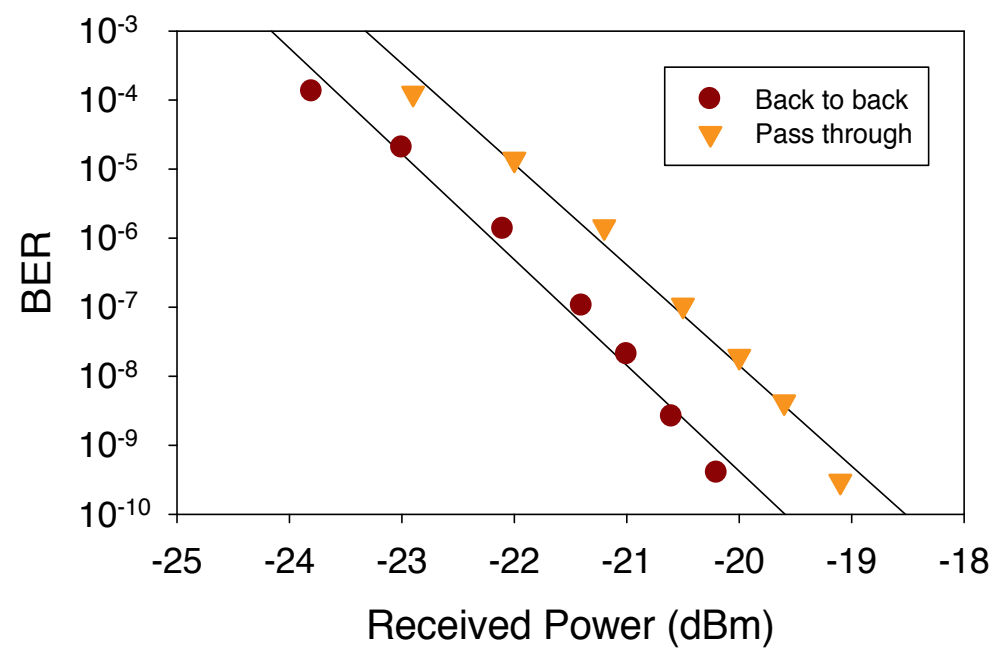

Figure 6. Performance of BER at a test signal of $1506 \mathrm{~nm}$ in a $2.5 \mathrm{Gbit} / \mathrm{s}$ modulation system, when a $980 \mathrm{~nm}$ pump power is set at $280 \mathrm{~mW}$. 
To investigate the output performance of proposed S-band gain-clamped EDFA module, a bit error rate (BER) measurement is performed. In the measurement, we utilize the optical CP of $80 \%$ input coupling ratio and FBG of $1517.37 \mathrm{~nm}$ wavelength for the proposed gain-clamped EDFA module. We use a test input signal of $1506 \mathrm{~nm}$ and modulate externally by using an $\mathrm{LiNbO}_{3}$ electro-optical (EO) modulator with $2.5 \mathrm{Gbit} / \mathrm{s}$ nonreturn-to-zero pseudorandom binary sequence (NRZ-PRBS) under a pattern length of $2^{31}-1$. However, the BER of back-toback (B2B) status is without utilizing proposed EDFA. The $2.5 \mathrm{GHz}$ PIN-based receiver (Rx) is used to detect the testing signal. Figure 6 presents the BER performance of proposed S-band EDFA under different received powers at the B2B status and the testing signal through the gain-clamped EDFA. Here, other input channels are also added or dropped into the gainclamping EDFA. When a testing input signal transmits through the proposed EDFA, the power penalty of $\sim 1 \mathrm{~dB}$ can be retrieved at the BER of $10^{-9}$.

\section{C-band gain-flattened EDFA module}

In this section, we will introduce a gain-flattened two-stage erbium-based fiber amplifier (EBFA) module, constructed by an erbium-doped waveguide amplifier (EDWA) and an EDFA in serial structure. Hence, the obtained gain profile of proposed amplifier not only enhances its value, but also possesses flatness performance. In addition, the output performance of the gain and NF in the EBFA has also been discussed.

Here, the proposed gain-flattened two-stage erbium-based fiber amplifier (EBFA) in serial configuration is illustrated in Fig. 7. The first and second stages are EDWA and EDFA. Besides, two tunable laser sources are employed to serve as a saturation tone and a probe tone, respectively, for gain and NF measurements. An optical spectrum analyzer (OSA) with a 0.05 $\mathrm{nm}$ resolution is utilized to measure the gain and NF. On account of the homogeneously broadened gain distribution, the multi-wavelength input signal would be simulated by applying a saturation signal with a saturated power equals to the collected power of multiwavelength input signal. Besides, the substantial spectral-hole burning is obtained around the wavelength of $1550 \mathrm{~nm}$; we can set a saturation signal at $1535 \mathrm{~nm}$ with $-25 \mathrm{dBm}$ in the measurement for simulating the multi-wavelength signals. The probe light should be smaller than $20 \mathrm{~dB}$ compared to the saturating signal.

In the measurement, the EDWA, which is produced via a two-step ion-exchange process, has the benefit of inheriting the known characteristics of EDFA such as, low NF, low polarization dependence, and without cross talk between the WDM wavelengths. All of the optical implementations are measured when a bias current of $980 \mathrm{~nm}$ pumping laser diode (LD) is 440 $\mathrm{mA}$ at ambient temperature. Besides, optical ISOs can reduce backward amplified spontaneous emission (ASE) and improve NF performance, and the pump kill filter is utilized to eliminate $980 \mathrm{~nm}$ pump power and keep $1550 \mathrm{~nm}$ signal pass. Figure 8 shows the gain and noise figure spectra of EDWA for $-25 \mathrm{dBm}$ input saturation power in an operating range of 1528-1562 nm. However, the measured gain and NF of 30.1 and $5.7 \mathrm{~dB}$ also are observed at $1532 \mathrm{~nm}$, and the NFs are between 5 and $6.3 \mathrm{~dB}$ in the wavelengths of $1524-1572 \mathrm{~nm}$, when the input saturation 


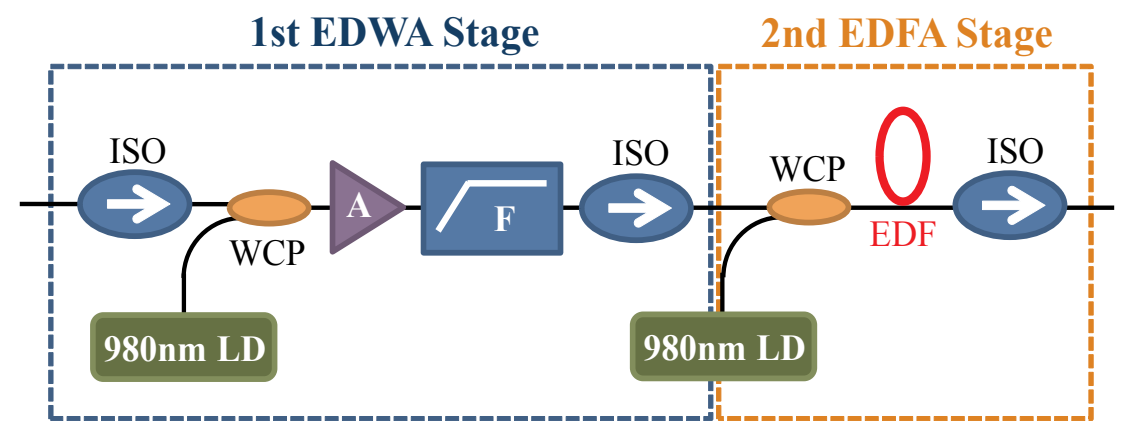

Figure 7. Proposed two-stage gain-flattened EBFA module, which is structured by an EDWA and an EDFA in serial.

power is $-25 \mathrm{dBm}$. The maximum gain variation of $4.8 \mathrm{~dB}$ is also retrieved in Fig. 8 in the wavelength range of $1524-1562 \mathrm{~nm}$.

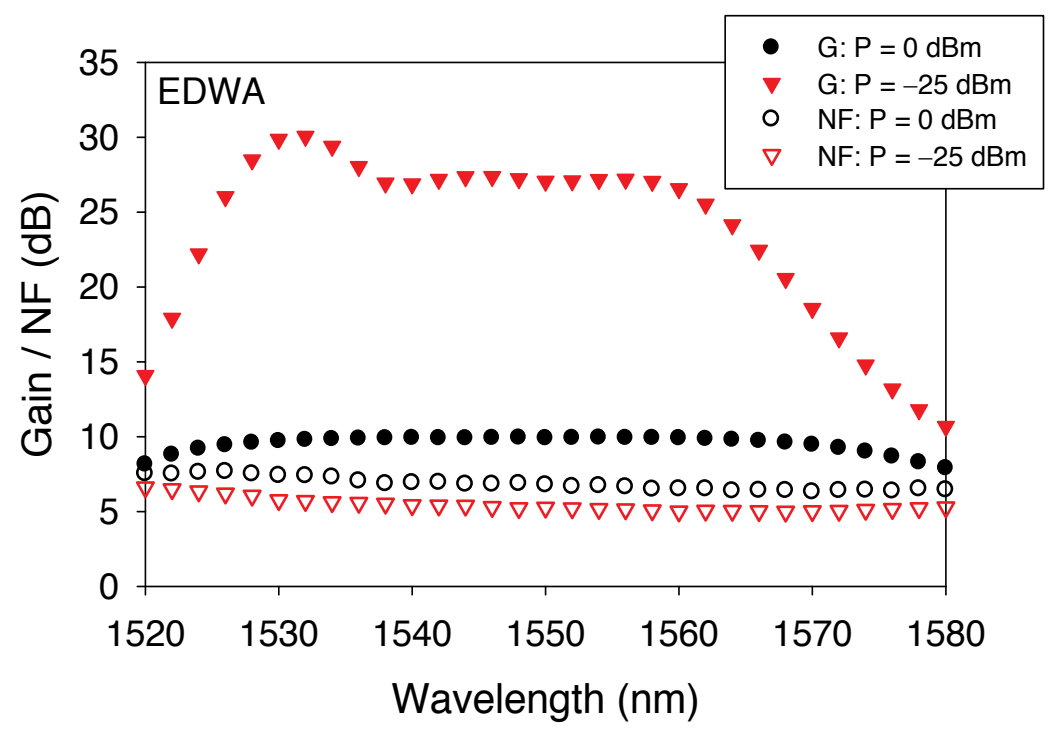

Figure 8. Gain and noise figure spectra of the EDWA in a bandwidth of $1520-1580 \mathrm{~nm}$ for $-25 \mathrm{dBm}$ input saturation power.

The second EDFA stage is constructed by an EDF of $10 \mathrm{~m}$, a $980 \mathrm{~nm}$ pumping LD, a 980/1550 $\mathrm{nm}$ WDM coupler (WCP), and an OIS. The pumping power of $980 \mathrm{~nm}$ LD is set at $72 \mathrm{~mW}$. Figure 9 displays the measured gain and NF spectra of EDFA when the $-25 \mathrm{dBm}$ input saturation power is used in the wavelengths of 1528-1562 nm. Furthermore, the peak gain and $\mathrm{NF}$ of $36.2 \mathrm{~dB}$ and $4.8 \mathrm{~dB}$ are observed at the wavelength of $1532 \mathrm{~nm}$ for the input saturation power of $-25 \mathrm{dBm}$. Here, the maximum gain difference of $12.2 \mathrm{~dB}$ is also observed in a wavelength region of 1524-1562 nm, as seen in Fig. 9. 


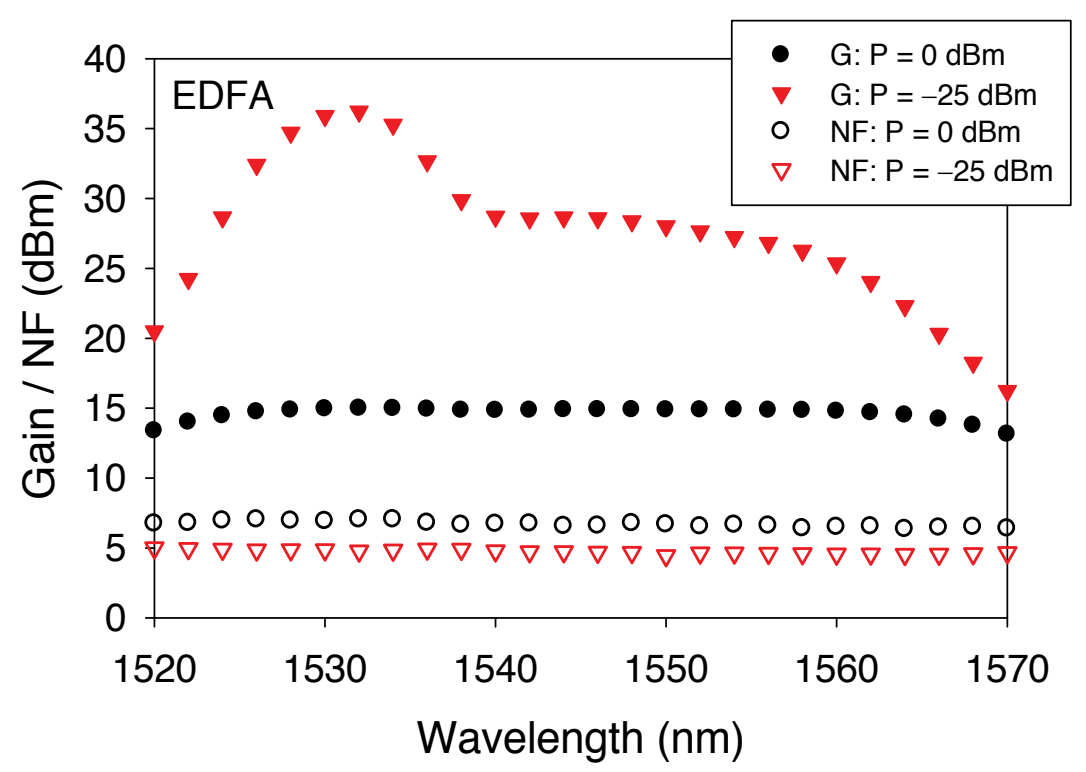

Figure 9. Gain and NF spectra of the EDFA with $10 \mathrm{~m}$ long EDF length in a bandwidth of $1520-1580 \mathrm{~nm}$ for $-25 \mathrm{dBm}$ input saturation power with the pump power of $72 \mathrm{~mW}$.

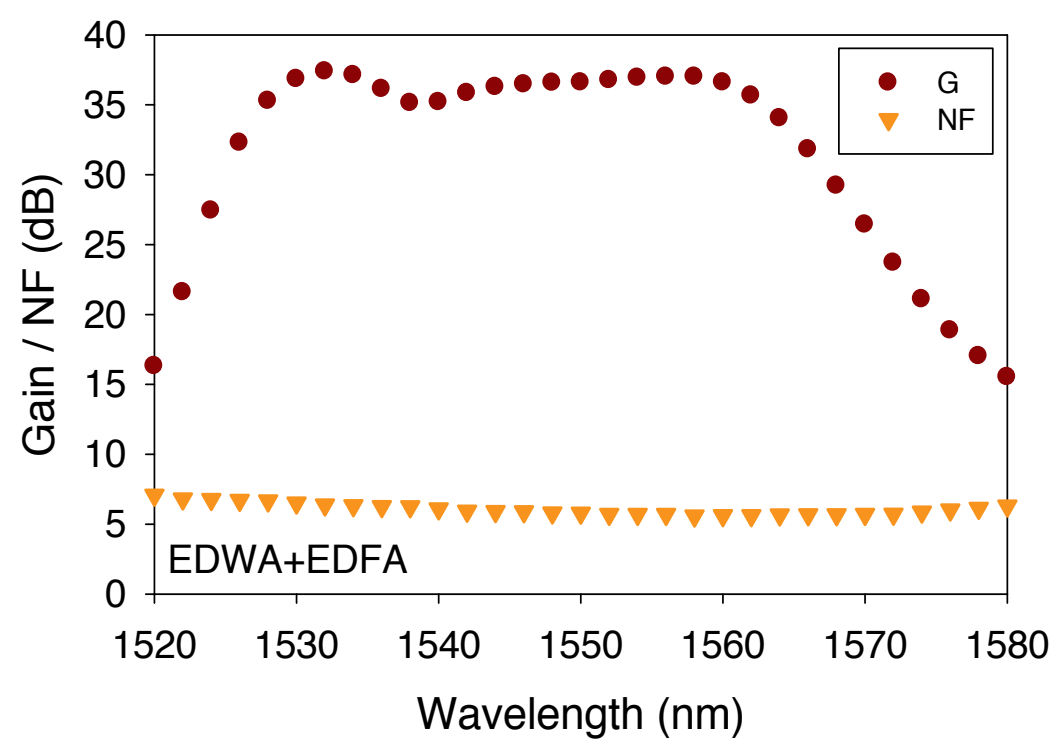

Figure 10. Gain and NF spectra of the proposed gain-flattened two-stage EBFA in a bandwidth of 1520-1580 nm for -25 $\mathrm{dBm}$ input saturation power. 


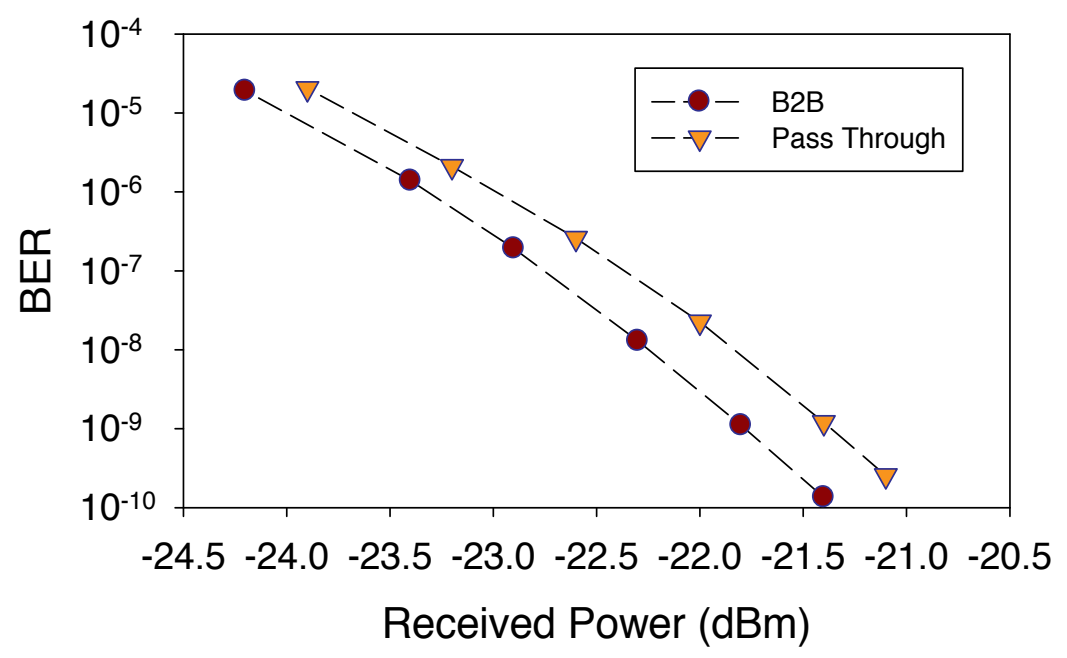

Figure 11. Performance of BER at a test signal of $1550 \mathrm{~nm}$ in $2.5 \mathrm{Gbit} / \mathrm{s}$ modulated system for the back-to-back type and proposed gain-flattened EBFA module.

To realize the gain-flattened amplifier, a two-stage EBFA module is illustrated in Fig. 7 for this experiment. The workable mechanism is possibly the gain saturation effect of EDWA and EDFA to accomplish the gain-flattening output. Thus, Fig. 10 displays the measured gain and NF profiles of the proposed gain-flattened EBFA, when $-25 \mathrm{dBm}$ input saturation power is utilized in the wavelength range of 1528-1562 nm. Figure 10 shows two maximum gains of 37.4 and $37.0 \mathrm{~dB}$, observed at the wavelengths of 1532 and $1556 \mathrm{~nm}$, respectively. The maximal gain difference of $\pm 1.1 \mathrm{~dB}$ could be also measured. According to the above results, this gainflattened EBFA. The proposed EBFA can approach the gain-flattening and also enhance the gain value due to the gain saturation effect and two-stage amplifier. Thus, the EBFA module increases the entire gain (all $>35 \mathrm{~dB}$ ) in the wavelength range of $1528-1562 \mathrm{~nm}$, and the gain spectrum can fix the flatness with the maximum variation of $\pm 1.1 \mathrm{~dB}$ for the input saturation power of $-25 \mathrm{dBm}$. Generally, the gain-flattened EDFAs could employ the various optical filters for filtering the redundant ASE to maintain the flattening output. However, the previous related technologies could bring the loss and the gain degradation. Therefore, the proposed EBFA not only can flatten the output gain spectrum, but can also increase the gain performance.

Here, a BER measurement is also performed in this experiment. The testing input signal at $1550 \mathrm{~nm}$ is modulated by using an EO modulator with 2.5 Gbit/s NRZ-PRBS with a pattern word of $2^{31}-1$. Here, we use a $2.5 \mathrm{GHz}$ PIN-based receiver to detect the testing signal. Figure 11 presents the measured BER of the proposed optical amplifier against the received power in the B2B status and passing through the gain-flattened EBFA module. In the measurement, when a testing input signal passes through the amplifier module, the observed optical power penalty is $\sim 0.4 \mathrm{~dB}$, while the BER is $10^{-9}$. 


\section{C- to L-bands optical fiber amplifier module}

In this section, we experimentally investigate a broadband hybrid two-stage S- to L-band fiber amplifier in serial configuration employing a C-band EDFA to cascade a C-band semiconductor optical amplifier (SOA). Hence, the proposed amplifier could achieve a $110 \mathrm{~nm}$ amplification bandwidth from 1500 to $1610 \mathrm{~nm}$, when the preamplifier only has a $3 \mathrm{~m}$ long EDF length. In addition, the output performance of the gain and NF for the proposed wideband amplifier has also been analyzed and discussed.

Here, the proposed hybrid two-stage S- to L-band fiber amplifier in serial is illustrated in Fig. 12. The proposed amplifier consists of an EDFA and a SOA in serial configuration. The first EDFA stage with preamplification function is used to reduce the NF value and improve the operated range of gain. Besides, the pumping current of second SOA stage is operated at 150 $\mathrm{mA}$. The threshold and maximum currents of the SOA used are $50 \mathrm{~mA}$ and $250 \mathrm{~mA}$, respectively. The SOA can be used in bidirection transmission. In Fig. 12, the ISO is employed to prevent the backward ASE power of SOA launched into the first amplifier stage.

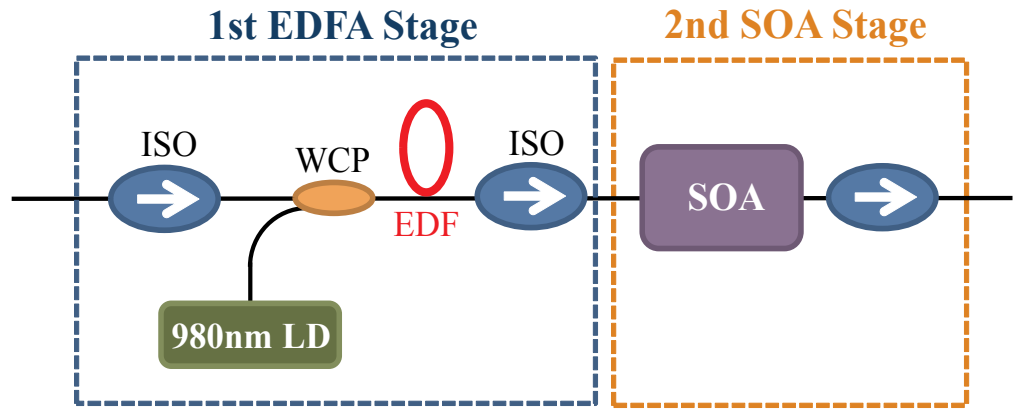

Figure 12. Proposed hybrid two-stage S- to L-band fiber amplifier, structured by an EDFA and an SOA in serial.

Fig. 13 shows the gain and NF spectra of an SOA, when the pumping current is $150 \mathrm{~mA}$ and the input saturation powers are 0 and $-25 \mathrm{dBm}$, respectively, in a wavelength range of 1520 $1600 \mathrm{~nm}$. Besides, a saturated power of the SOA could be up to $11.1 \mathrm{dBm}$ for $0 \mathrm{dBm}$ input saturation power at $1548 \mathrm{~nm}$. And $23.6 \mathrm{~dB}$ maximum gain and $7.5 \mathrm{~dB} \mathrm{NF}$ is observed at 1520 $\mathrm{nm}$ when the input saturation power is $-25 \mathrm{dBm}$, as seen in Fig. 13. As illustrated in Fig. 13, the NF spectra of the SOA are between 8.2 and $7.2 \mathrm{~dB}$, and 7.5 and $6.6 \mathrm{~dB}$ when the input saturation power is 0 and $-25 \mathrm{dBm}$, respectively, in the bandwidth of $1520-1600 \mathrm{~nm}$. According to experimental results, the SOA presents the lower gain and worse noise figure in C-band. Owing to these defects of the SOA, it cannot be employed in optical communication system for amplification. To solve these drawbacks, an EDFA with preamplification function is applied in the proposed broadband fiber amplifier. However, to realize the impact of EDF length for the first stage, we would utilize various EDF lengths in the experiment. In general, an ideal optical amplifier needs to have the advantages of broadband amplification range, higher gain, lower NF, etc. 


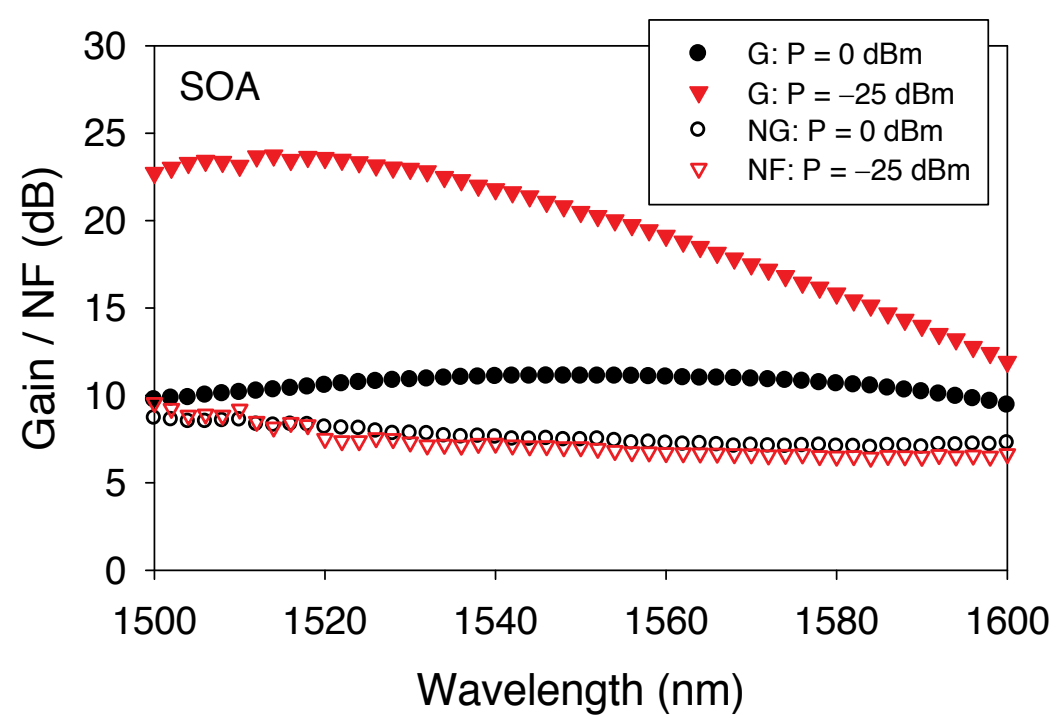

Figure 13. Gain and NF spectra of an SOA, when the pumping current operates at $150 \mathrm{~mA}$ and the input saturation power is 0 and $-25 \mathrm{dBm}$, respectively, in the operating range of $1520-1600 \mathrm{~nm}$.

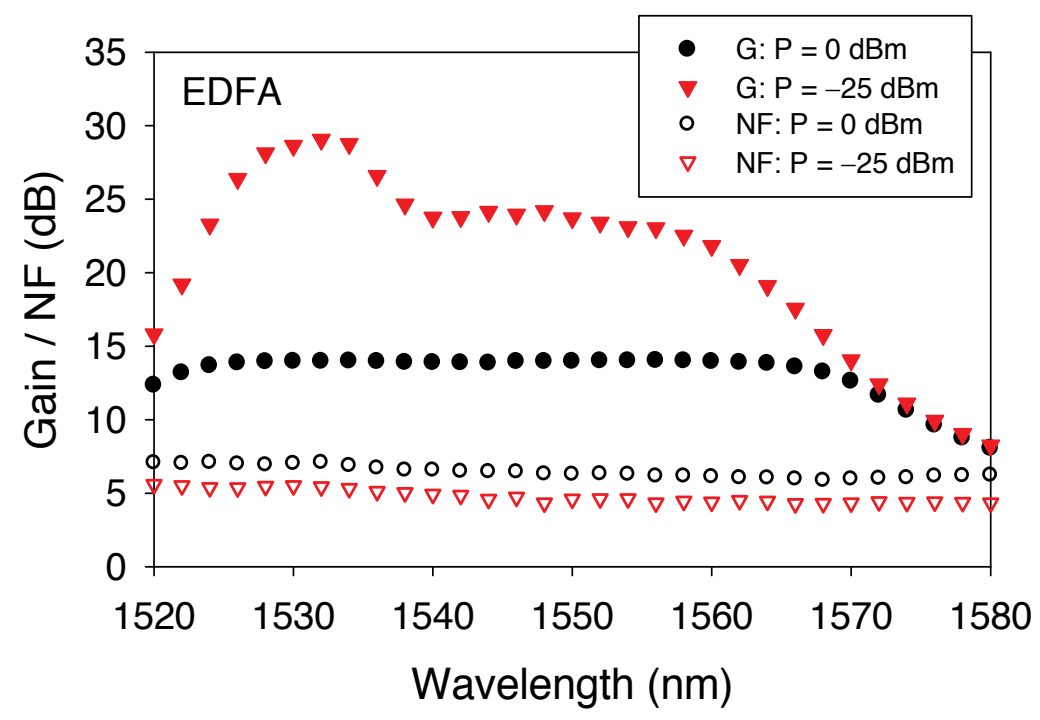

Figure 14. Gain and NF spectra of an EDFA when the input saturation power $P_{\text {sat }}=0$ and $-25 \mathrm{dBm}$ in the wavelength of $1520-1570 \mathrm{~nm}$. The length of EDF is $10 \mathrm{~m}$ long and the pumping power of $980 \mathrm{~nm}$ laser is $60 \mathrm{~mW}$.

First, a $10 \mathrm{~m}$ long EDF (DF-1500F of Fibercore Ltd.) length is utilized in the first EDFA stage with a $60 \mathrm{~mW}$ pump power. Figure 14 shows the gain and NF spectra of the original EDFA for 


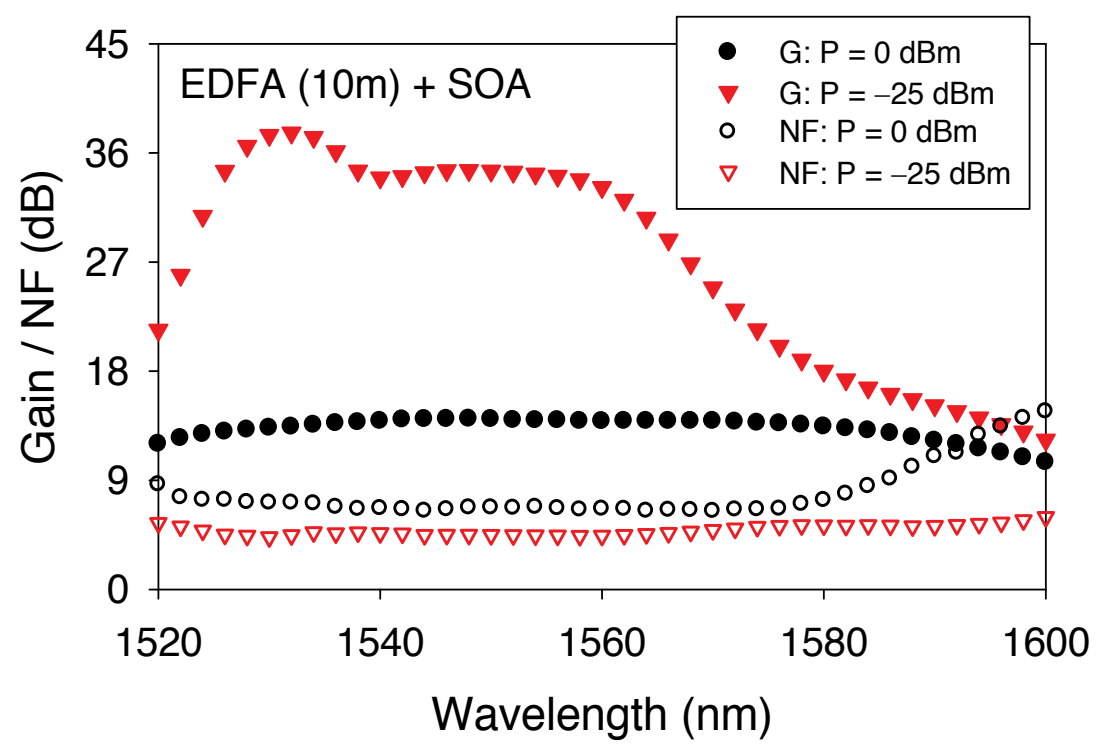

Figure 15. Measured gain and NF profiles of the hybrid two-stage fiber amplifier module (with $10 \mathrm{~m}$ long EDF length).

the input saturation power $P_{\text {sat }}=0$ and $-25 \mathrm{dBm}$ in an operating range of $1520-1570 \mathrm{~nm}$. Also shown are the peak gain and NF of 29.1 and $5.4 \mathrm{~dB}$ at $1532 \mathrm{~nm}$, when the input saturation power is $-25 \mathrm{dBm}$. Figure 14 also illustrates that all the gain is higher than $12 \mathrm{~dB}$ and the NF is less than $7.2 \mathrm{~dB}$ at the above-mentioned operating conditions from 1520 to $1570 \mathrm{~nm}$.

When an EDFA with a $10 \mathrm{~m}$ EDF and $60 \mathrm{~mW}$ pumping power is used to cascade an SOA with $150 \mathrm{~mA}$ pumping current, the gain and NF profiles of the hybrid amplifier for the input saturation power $P_{\text {sat }}=0$ and $-25 \mathrm{dBm}$ in the wavelengths of $1520-1600 \mathrm{~nm}$ is illustrated in Fig. 15. The entire gain and noise figure of the hybrid amplifier are improved between around Cband, but the effectively operating range becomes narrower compared with the original Cband SOA. The saturated power could achieve $14.1 \mathrm{dBm}$ for the input saturation power of 0 $\mathrm{dBm}$ at $1548 \mathrm{~nm}$. In Fig. 15, the $37.7 \mathrm{~dB}$ peak gain and $4.6 \mathrm{~dB}$ NF are retrieved at $1532 \mathrm{~nm}$ when the input saturation power is $-25 \mathrm{dBm}$. Figure 15 also shows that the noise figure is distributed between 6.3 and $14.8 \mathrm{~dB}$ and 4.4 and $5.9 \mathrm{~dB}$ in the operating range of 1520-1600 $\mathrm{nm}$ for the input power $\mathrm{P}_{\text {sat }}=0$ and $-25 \mathrm{dBm}$, respectively. From the observed results, those are not enough good in our expectancy.

Then, we decrease the EDF length to $3 \mathrm{~m}$ in the first EDFA stage (with a $40 \mathrm{~mW}$ pumping power) to connect with the second SOA stage (with $150 \mathrm{~mA}$ pumping current) in series. Figure 16 shows the gain and NF profiles of the proposed two-stage amplifier in the operation bandwidth from 1500 to $1610 \mathrm{~nm}$, when the input saturation power $\left(\mathrm{P}_{\text {sat }}\right)$ is 0 and $-25 \mathrm{dBm}$, respectively. Figure 16 also shows that the $13.7 \mathrm{dBm}$ saturated power at $1558 \mathrm{~nm}$ is obtained for $0 \mathrm{dBm}$ input saturation power, and a maximum gain of $35.3 \mathrm{~dB}(4.3 \mathrm{~dB} \mathrm{NF})$ at $1532 \mathrm{~nm}$ is retrieved for $-25 \mathrm{dBm}$ input saturation power. Based on the proposed architecture, the effectively operating range of the amplifier will achieve a $110 \mathrm{~nm}$ amplification bandwidth 
from 1500 to $1610 \mathrm{~nm}$. The new proposed structure not only enhances the gain value, but also extends the operating bandwidth from 1500 to $1610 \mathrm{~nm}$ (S- to L-bands). As a result, the proposed two-stage amplifier has the advantage of simple architecture design, $110 \mathrm{~nm}$ broadband amplification region, higher gain, and lower NF.

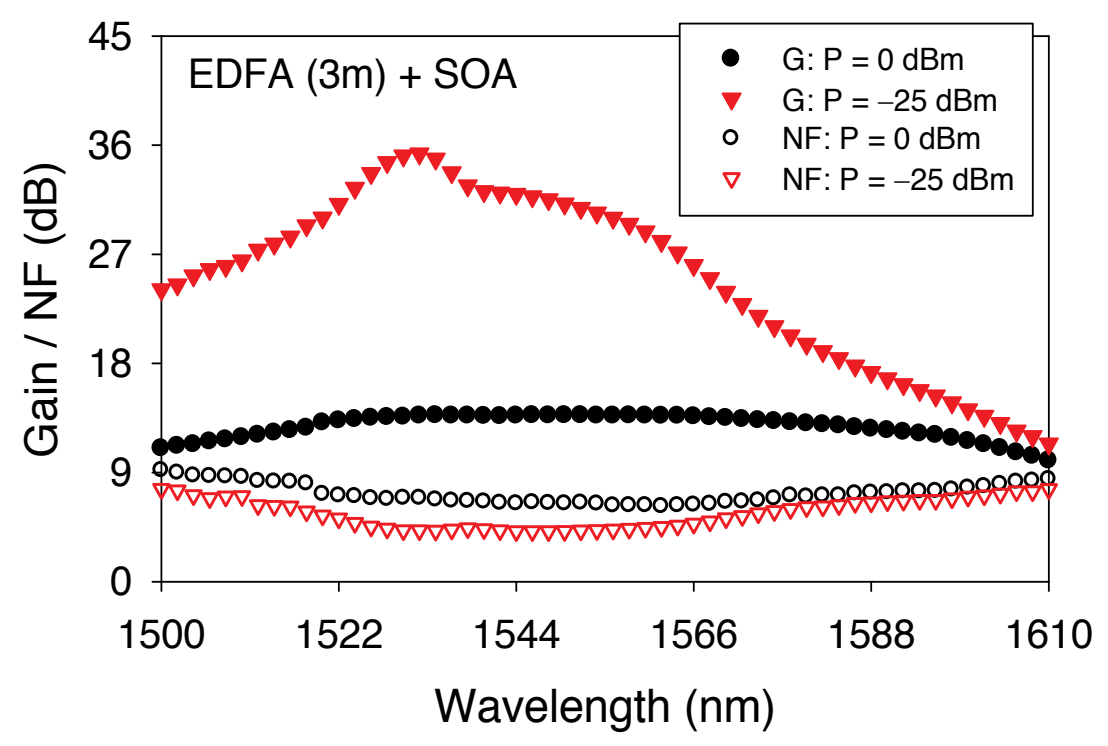

Figure 16. Measured gain and noise figure spectra of the hybrid two-stage fiber amplifier module (with $3 \mathrm{~m}$ long EDF length).

\section{Conclusion}

In summary, there are three sections that describe the broadband S-band to L-band erbiumdoped fiber amplifier modules. In the first part, an S-band gain-clamped erbium-doped fiber EDFA module, employing an FBG to act as a reflected element for generating a saturated tone injected into the EDFA module by using forward optical feedback method, is proposed. Moreover, the output performance of the gain and noise figure (NF) in the proposed gainclamped S-band EDFA has been discussed in the wavelength range of 1478-1520 nm.

In the second part, we propose and investigate a gain-flattened two-stage EBFA module, which is structured by an EDWA and a C-band EDFA in serial structure. In an operation range of $1528-1562 \mathrm{~nm}$, the entire gain is larger than $35 \mathrm{~dB}$ and the observed NF is between 5.5 and 6.7 $\mathrm{dB}$. Moreover, $\pm 1.1 \mathrm{~dB}$ maximum gain variation is also obtained for the input signal power of $-25 \mathrm{dBm}$. Hence, the proposed fiber amplifier not only enhances the gain but also achieves the flatness in the wavelength region. 
In the final part, a broadband hybrid two-stage fiber amplifier, which is constructed by a Cband EDFA and a C-band SOA in serial scheme, is investigated experimentally. Here, we only use a $3 \mathrm{~m}$ long erbium-doped fiber (EDF) serving as a preamplifier to increase the gain and reduce the noise figure. Therefore, the proposed hybrid amplifier achieves a $110 \mathrm{~nm}$ effectively amplification of 1500 to $1610 \mathrm{~nm}$ from S- to L-band. Moreover, the output performance of gain and NF in the proposed fiber amplifier has also been discussed.

\section{Acknowledgements}

This work was supported by the Ministry of Science and Technology, Taiwan, under grant MOST-103-2218-E-035-011-MY3.

\section{Author details}

\section{Chien-Hung Yeh*}

Address all correspondence to: yehch@fcu.edu.tw

Department of Photonics, Feng Cha University, Taichung, Taiwan

\section{References}

[1] B. Clesca, "Flat-gain amplifiers and transmission in WDM networks," Tech. Dig. Optical Amplifiers and their Applications, Monterey, CA, Paper FA1, pp. 54-57 1996.

[2] C. Jiang, W. Hu, Q. Zeng, and S. Xiao, "Novel split-band erbium-doped fiber amplifier," Opt. Laser Technol., vol. 35, pp. 251-256, 2003.

[3] C. H. Yeh, C. C. Lee, and S. Chi, "120-nm bandwidth erbium-doped fiber amplifier module in parallel configuration," IEEE Photonics Technol. Lett., vol. 16, pp. 16371639, 2004.

[4] Y. H. Lu and S. Chi, "All-optical gain-clamped wideband serial EDFA with ringshaped laser," Opt. Commun., vol. 229, pp. 317-323, 2004.

[5] Q. F. Jiang, X. M. Liu, Q. Wang, and X. Feng, "Dynamically gain control in the serial structure C+L wideband EDFA," IEEE. Photonics Technol. Lett., vol. 16, pp. 87-89, 2004.

[6] X. Fang, T. Jin, Y. Wang, X. Liu, and J. Peng, "A simple algorithm for wide-band channel-power clamped EDFA," Opt. Commun., vol. 213, pp. 285-292, 2002. 
[7] Y. H. Lu, L. H. Su, and Y. K. Chen, "Optically gain-clamped wideband erbium-doped fiber amplifier using a common figure-eight feedback-loop lasing light," Opt. Commun., vol. 229, pp. 293-298, 2002.

[8] K. Inoue, "Gain-clamped fiber amplifier with a loop mirror configuration", IEEE Photonics Technol. Lett., 5, 533-535, 1999.

[9] M. Cai, X. Liu, J. Cui, P. Tang, D. Liu, and J. Peng, "Study on noise characteristic of gain-clamped erbium-doped fiber-ring lasing amplifier," IEEE Photonics Technol. Lett., vol. 9, pp. 1093-1095, 1997.

[10] C. H. Yeh, M. C. Lin, T. T. Huang, K. C. Hsu, C. H. Ko, and S. Chi, "S-band gainclamped grating-based erbium-doped fiber amplifier by forward optical feedback technique," Opt. Express, vol. 14, no. 7, pp. 2611-2617, 2006.

[11] M. A. Arbore, Y. Zhou, G. Keaton, and T. J. Kane, “30 dB gain at 1500 nm in S-band Erbium-doped silica fiber with distributed ASE suppression," in Proc. SPIE, Optical Devices for Fiber Communication IV, 4989, pp. 47-52, 2003.

[12] M. Yamada, T. Kanamori, Y. Terunuma, K. Oikawa, M. Shimizu, S. Sudo, and K. Sagawa, "Fluoride-based erbium-doped fiber amplifier with inherently flat gain spectrum," IEEE Photon. Technol. Lett., vol. 8, pp. 882-884, 1996.

[13] M. K. Pandit, K. S. Chiang, Z. H. Chen, and S. P. Li, “Tunable long period fiber gratings for EDFA gain and ASE equalization," Microwave Opt. Technol. Lett., vol. 25, pp. 181-184, 1999.

[14] P. F. Wysocki, J. B. Judkins, R. P. Espindola, M. Andrejco, and A. M. Vengsarkar, "Broad-band erbium-doped fiber amplifier flattened beyond $40 \mathrm{~nm}$ using long-period grating filter," IEEE Photon. Technol. Lett., vol. 9, pp. 1343-1345, 1997.

[15] S. K. Liaw, K. P. Ho, and S. Chi, "Dynamic power-equalized EDFA module based on strain tunable fiber Bragg gratings," IEEE Photon. Technol. Lett., vol. 11, pp. 797-799, 1999.

[16] R. Feced, C. Alegria, M. N. Zervas, and R. I. Laming, "Acoustooptic attenuation filters based on tapered optical fibers," IEEE J. Select. Top. Quantum Electron., vol. 5, no. 3, pp. 1278-1288, 1999.

[17] S. K. Yun, B. W. Lee, H. K. Kim, and B. Y. Kim, “Dynamic erbium-doped fiber amplifier based on active gain flattening with fiber acoustooptic tunable filter," IEEE Photon. Technol. Lett., vol. 11, pp. 1229-1231, 1999.

[18] J. Nilsson, W. H. Loh, S. T. Hwang, J. P. de Sandro, and S. J. Kim, “Simple gain-flattened erbium-doped fiber amplifier with a wide dynamic range," in Opt. Fiber Commun. Conf. Washington, DC: Optical Society of America, 1997, OSA Tech. Dig., p. 163. 
[19] R. A. Betts, S. J. Frisken, and D. Wong, "Split-beam Fourier filter and its application in a gain-flattened EDFA," in Opt. Fiber Commun. Conf., Washington, DC: Opt. Soc. Amer., 1995, OSA Tech. Dig. Series, pp. 80-81.

[20] C.-H. Yeh, M.-C. Lin, and S. Chi, "Gain flattened erbium-doped amplifier with $34 \mathrm{~nm}$ flat bandwidth," Electronics Letters, vol. 42, no. 19, pp. 1086-1088, September 2006.

[21] Y. Sun, J. W. Sulhoff, A. K. Srivastava, et. al., "80nm ultra-wideband erbium-doped silica fiber amplifier," Electron. Lett. 33, 1965-1967 (1997).

[22] H. Masuda, S. Kawai, K. I. Suzuki and K. Aida, "Wideband, gain-flattened, erbiumdoped fiber amplifiers with 3dB bandwidths of $>50 \mathrm{~nm}$," Electron. Lett. 33, 1070-1072 (1997).

[23] B. Min, H. Yoon, W. J. Lee, and N. Park, "Coupled structure for wide-band EDFA with gain and noise figure improvements from C to L-band ASE injection," IEEE. Photonics Technol. Lett. 12, 480-482 (2000).

[24] R. Di Muro, D. Lowe, and S. Wilson, "Broad-band amplification using a novel amplifier topology," IEEE. Photonics Technol. Lett. 13, 1073-1075 (2001).

[25] S. Hwang, K. W. Song, et al. "Comparative high power conversion efficiency of Cplus L-band EDFA," Electron. Lett. 37, 1539-1541 (2001).

[26] Y. H. Lu, S. Chi, "All-optical gain-clamped wideband serial EDFA with ring-shaped laser," Opt. Commun. 229, 317-323 (2004).

[27] Q. F. Jiang, X. M. Liu, Q. Wang, and X. Feng, “Dynamically gain control in the serial structure C+L wide-band EDFA," IEEE. Photonics Technol. Lett. 16, 87-89 (2004).

[28] C.-H. Yeh and S. Chi, "Utilizations of EDFA and SOA in series for broadband gain amplification," Laser Phys. Lett., vol. 4, pp. 433-436, 2007.

[29] C. H. Yeh, C. C. Lee and S. Chi, "S- plus C-band erbium-doped fiber amplifier in parallel structure," Opt. Commun., vol. 241, pp. 443-447, 2004.

[30] S. W. Harun, N. Tamchek, P. Poopalan, and H. Ahamd, "Double-pass L-band with enhanced noise figure characteristics," IEEE. Photonics Technol. Lett., vol. 15, pp. 1055-1057, 2003.

[31] L. L. Yi, L. Zhan, J. H. Ji, Q. H. Ye, and Y. X. Xia, "Improvement of gain and noise figure in double-pass L-band EDFA by incorporating a fiber Bragg grating," IEEE Photonics Technol. Lett., vol. 16, pp. 1005-1007, 2004.

[32] H. B. Choi, J. M. Oh, and D. Lee, "Simple and efficient L-band erbium-doped fiber amplifiers for WDM networks," Opt. Commun., vol. 213, pp. 63-66, 2002.

[33] C.-H. Yeh, C.-C. Lee, and S. Chi, "A tunable S-Band erbium-doped fiber ring laser," IEEE Photon. Technol. Lett., vol. 15, pp. 1503-1504, 2003. 

Chapter 7

\title{
Optimized Design of $\mathrm{Yb}^{3+} / \mathrm{Er}^{3+}$-Codoped Phosphate Microring Resonator Amplifiers
}

\author{
Juan A. Vallés and R. Gălătuş \\ Additional information is available at the end of the chapter \\ http://dx.doi.org/10.5772/61767
}

\begin{abstract}
A precise model to numerically analyse the performance of a highly $\mathrm{Yb}^{3+} / \mathrm{Er}^{3+}$-codoped phosphate glass microringresonator (MRR) is presented. This model assumes resonant behaviour inside the ring for both pump and signal powers and considers the coupled evolution of the rare earth (RE) ions population densities and the optical powers that propagate inside the MRR. Energy-transfer inter-atomic processes that become enhanced by required high-dopant concentrations have to be carefully considered in the numerical design. The model is used to calculate the performance of an active add-dropfilter and the more significant parameters are analysed in order to achieve an optimized design. Finally, the model is used to determine the practical requirements for amplification and oscillation in a highly $\mathrm{Yb}^{3+} / \mathrm{Er}^{3+}$-codoped phosphate glass MRR side-coupled to two straight waveguides for pump and signal input/output. In particular, the influence of dopant concentration, additional coupling losses and the structure symmetry are fully discussed.
\end{abstract}

Keywords: Active integrated microring resonators, $\mathrm{Yb}^{3+} / \mathrm{Er}^{3+-}$ codoped glass, energy-transfer inter-atomic mechanisms, gain/ oscillation requirements, asymmetric structures

\section{Introduction}

Microring resonators (MRR) have attracted much attention as multifunctional components for signal processing in optical communication systems [1-4]. Recently, due to their fabrication scalability, functionalization and easiness in sensor interrogation, MRR with chip-integrated linear access waveguides have emerged as promising candidates for scalable and multiplexable sensing platforms, providing label-free, highly sensitive and real-time detection capabilities [5-8]. The near-infrared spectral range and, in particular, the $1.5-\lambda \mathrm{m}$ wavelength band is already employed in several bio-/chemical sensing tasks using MRR [9-11]. 
If gain is incorporated inside the ring, losses (intrinsic absorption, scattering, bend, etc.) can be compensated, filtering and amplifying/oscillating functionalities are combined [12,13] and the sensing potentialities of the device become enhanced [14]. Due to their excellent spectroscopic and solubility characteristics, phosphate glass is a suitable host for rare earth (RE) high doping and $\mathrm{Yb}^{3+} / \mathrm{Er}^{3+}$-codoped phosphate glass integrated waveguide amplifiers and lasers provide a compact, efficient and stable performance [15]. However, when the host material of an MRR is $\mathrm{Yb}^{3+} / \mathrm{Er}^{3+}$-codoped, the modelling of the performance of the active structure becomes much more complex, since the coupled evolution of the optical powers and the rare earth (RE) ions population densities has to be properly described. Moreover, $\mathrm{Er}^{3+}$-ion efficiency-limiting energy-transfer inter-atomic interactions (homogeneous upconversio $n$ and migration), which are enhanced by high RE-doping levels required by the device dimensions, have to be considered for an optimized design [16].

In the literature, a few models describing RE-doped microfiber ring lasers can be found [17], but there the dopant concentrations level was much lower than those needed in MRRs. Additionally, a simplified model for RE-doped MRR has been proposed where the energytransfer mechanisms were directly ignored [18]. In a previous paper, we incorporated the effect of high dopant concentrations and presented some results of the optimized active performance of this device [19]. However, in that paper not only the gain coefficient was averaged along the amplifier total length but also the pump resonant behaviour inside the ring and the influence of coupler additional losses were neglected. In subsequent papers we developed a much more detailed model of the performance of a highly $\mathrm{Yb}^{3+} / \mathrm{Er}^{3+}$-codoped phosphate glass add-dropfilter filter that overcame previous models deficiencies. An active MRR was described by using a formalism for the intensity rates of the optical powers (pump and signal) at resonance affected by their interaction with the dopant ions through absorption/emission processes. Thus, the performance of an active MRR could be calculated in order to analyse its optimized design and to determine the conditions to achieve amplification and oscillation $[20,21]$.

Drop-port output power maximizing symmetrically coupled structures are mostly used in MRR-based passive components. Alternatively, asymmetric waveguide/MRR coupling may offer definite optimum functional behaviour [22]. For instance, with critically coupled MMRs, the highest throughput attenuation can be attained [23] or when MRR are used as dispersion compensators in the time domain [24].

In this chapter we present a review of our previous works in modelling of $\mathrm{Yb}^{3+} / \mathrm{Er}^{3+}$-codoped phosphate microring resonator amplifiers. First, in Section 2, a detailed model of the performance of a highly $\mathrm{Yb}^{3+} / \mathrm{Er}^{3+}$-codoped phosphate glass add-drop filter is presented. This model describes the coupled evolution of the rare earth ions population densities and the optical powers that propagate inside the MRR assuming a resonant behaviour inside the ring for both pump and signal powers. In order to exploit the active potentialities of the structure, high dopant concentrations are needed. Therefore, energy-transfer inter-atomic processes are included in the numerical design. The microscopic statistical formalism based on the statistical average of the excitation probability of the $\mathrm{Er}^{3+}$ ion in a microscopic level has been used to 
describe migration-assisted upconversion. Moreover, due to its high solubility for rare earth ions, phosphate glass is considered an optimum host.

In Section 3, the model is used to calculate the performance of an active microring resonator and the more significant parameters are analysed in order to achieve an optimized design. Finally, in Section 4, the model is used to determine the practical requirements for amplification and oscillation in a highly $\mathrm{Yb}^{3+} / \mathrm{Er}^{3+}$-codoped phosphate glass MRR side-coupled to two straight waveguides for pump and signal input/output. In particular, the influence of dopant concentration, additional coupling losses, and the structure symmetry are fully discussed.

\section{2. $\mathrm{Yb}^{3+} / \mathrm{Er}^{3+}$-codoped microring resonator model}

\subsection{Active integrated microring transfer functions}

An MRR evanescently coupled to two straight parallel bus waveguides (commonly termed an add-drop filter) is the structure under analysis (see Fig. 1). In our formalism, the add port is ignored since only amplifiers and laser amplifiers are considered. Clockwise direction, singlemode single-polarization propagation is considered. Moreover, the bus waveguides and the MRR are assumed to have the same complex amplitude propagation constant $\beta_{c}=\beta-j \alpha+j g$. In this expression, $\beta$ is the phase propagation constant, $\alpha$ is the loss coefficient (due to scattering and bend) and $g$ is the gain coefficient. This coefficient describes the evolution of the pump/ signal mode amplitudes caused by their interaction with the RE ions.

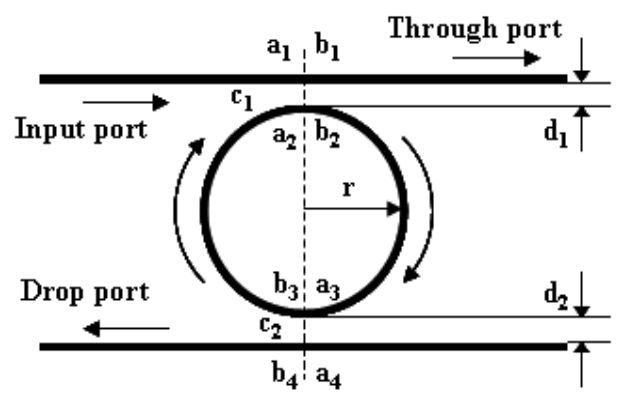

Figure 1. A microring resonator side-coupled to two parallel straight waveguides for pump and signal input/output. The scheme is not to scale.

In Fig. $1 \mathrm{r}$ is the microring radius and the central coupling gaps between each waveguide and the ring are $d_{i}(i=1,2)$. Lossless intensity coupling and transmission coefficients at coupler $c_{i}$ are $K_{i}^{0}$ and $T_{i}^{0}$, satisfying $K_{i}^{0}+T_{i}^{0}=1$. Correspondingly, $\kappa_{i}^{0}=\left(K_{i}^{0}\right)^{1 / 2}$ and $t_{i}^{0}=\left(T_{i}^{0}\right)^{1 / 2}$ are the lossless amplitude coupling and transmission coefficients. Realistically, we also consider additional coupling losses at the waveguide/microring couplers. Even small additional coupling losses may have a large influence on the MRR performance [20]. $\Gamma_{i}$ denotes the coefficient for additional intensity loss at the ith coupler. Therefore, the actual intensity 
coupling and transmission coefficients are $T_{i}=\left(1-\Gamma_{i}\right) T_{i}^{0}, K_{i}=\left(1-\Gamma_{i}\right) K_{i}^{0}$, which verify the relation $T_{i}+K_{i}=\left(1-\Gamma_{i}\right)$, whereas $t_{i}=T_{i}^{1 / 2}$ and $\kappa_{i}=K_{i}^{1 / 2}$ are the amplitude coupling and transmission coefficients, respectively. Mode confinement guarantees that interaction between the microring and bus waveguide cores is negligible outside the coupler regions.

Amplitudes at the couplers output ports. If the input/output complex amplitudes at the couplers ports are denoted as $a_{i}$ and $b_{i}$ ( $i=1,2$ for the input/through ports at coupler 1 and $i=3,4$ for the add/drop ports at coupler 2 , respectively) the following scattering matrix relations can be used to describe the exchange of optical power between the waveguides and the MRR:

$$
\text { Coupler I : }\left|\begin{array}{l}
b_{1} \\
b_{2}
\end{array}\right|=\left|\begin{array}{cc}
t_{1} & -j \kappa_{1} \\
-j \kappa_{1} & t_{1}
\end{array}\right|\left|\begin{array}{l}
a_{1} \\
a_{2}
\end{array}\right| ; \text { coupler II: }\left|\begin{array}{l}
b_{3} \\
b_{4}
\end{array}\right|=\left|\begin{array}{cc}
t_{2} & -j \kappa_{2} \\
-j \kappa_{2} & t_{2}
\end{array}\right|\left|\begin{array}{l}
a_{3} \\
a_{4}
\end{array}\right|
$$

and the relations between complex amplitudes at the directional couplers ports are:

$$
\begin{aligned}
& b_{1}=t_{1} a_{1}-j \kappa_{1} a_{2} \\
& b_{2}=-j \kappa_{1} a_{1}+t_{1} a_{2} \\
& b_{3}=t_{2} a_{3}-j \kappa_{2} a_{4} \\
& b_{4}=-j \kappa_{2} a_{3}+t_{2} a_{3}
\end{aligned}
$$

Moreover, the transmission along the two ring halves is such that

$$
\begin{aligned}
& a_{2}=b_{3} \exp (-j \varphi) \\
& a_{3}=b_{2} \exp (-j \varphi),
\end{aligned}
$$

where $\varphi=\pi r \beta_{c}$. Finally, if we assume that the only input signal is in the input port, the amplitudes at the output ports can be straightforwardly derived as:

$$
\begin{gathered}
b_{1}=\frac{t_{1}-\left(1-\Gamma_{1}\right) t_{2} \exp (-j 2 \varphi)}{1-t_{1} t_{2} \exp (-j 2 \varphi)} a_{1} \\
b_{2}=\frac{-j \kappa_{1}}{1-t_{1} t_{2} \exp (-j 2 \varphi)} a_{1}
\end{gathered}
$$




$$
\begin{aligned}
& b_{3}=\frac{-j \kappa_{1} t_{2} \exp (-j \varphi)}{1-t_{1} t_{2} \exp (-j 2 \varphi)} a_{1} \\
& b_{4}=\frac{-\kappa_{1} \kappa_{2} \exp (-j \varphi)}{1-t_{1} t_{2} \exp (-j 2 \varphi)} a_{1}
\end{aligned}
$$

Intensity rates. From Eqs (8)-(11) the input/output transfer functions of the structure in Fig. 1 , that is the rates of the intensities from the input port to the coupler output ports, can be readily obtained as follows:

$$
\begin{gathered}
I_{11}=\left|\frac{b_{1}}{a_{1}}\right|^{2}=\frac{t_{1}^{2}+\left(1-\Gamma_{1}\right)^{2} t_{2}^{2} \delta^{2}-2\left(1-\Gamma_{1}\right) t_{1} t_{2} \delta \cos (\beta L)}{1+t_{1}^{2} t_{2}^{2} \delta^{2}-2 t_{1} t_{2} \delta \cos (\beta L)} \\
I_{21}=\left|\frac{b_{2}}{a_{1}}\right|^{2}=\frac{\kappa_{1}^{2}}{1+t_{1}^{2} t_{2}^{2} \delta^{2}-2 t_{1} t_{2} \delta \cos (\beta L)} \\
I_{31}=\left|\frac{b_{3}}{a_{1}}\right|^{2}=\frac{\kappa_{1}^{2} t_{2}^{2} \delta}{1+t_{1}^{2} t_{2}^{2} \delta^{2}-2 t_{1} t_{2} \delta \cos (\beta L)} \\
I_{41}=\left|\frac{b_{4}}{a_{1}}\right|^{2}=\frac{\kappa_{1}^{2} \kappa_{2}^{2} \delta}{1+t_{1}^{2} t_{2}^{2} \delta^{2}-2 t_{1} t_{2} \delta \cos (\beta L)}
\end{gathered}
$$

where $L=2 \pi r$ is the length of the ring and $\delta=\exp [(g-\alpha) L]$ is the round-trip gain/loss. Mathematically, this structure is analogous to the classical Fabry - Perot interferometer. The output intensities at the through and drop ports correspond to its reflected and transmitted intensities, respectively. If the couplers are lossless, that is $\Gamma_{1}=\Gamma_{2}=0$, and there is no ring roundtrip loss, $\delta=1$, we obtain $I_{11}+I_{41}=1$. Moreover, if in Eq. (12) $\kappa_{2}=\Gamma_{2}=0$, and hence $t_{2}=1$, we obtain the through intensity rate of an all-pass ring resonator with only one coupler:

$$
\left|\frac{b_{1}}{a_{1}}\right|^{2}=\frac{t_{1}^{2}+\left(1-\Gamma_{1}\right)^{2} \delta^{2}-2\left(1-\Gamma_{1}\right) t_{1} \delta \cos (\beta L)}{1+t_{1}^{2} \delta^{2}-2 t_{1} \delta \cos (\beta L)}
$$

Finally, if the intensity rates are considered at the output ends of the straight waveguides, the amplitude evolution from/to the coupler output ports along the add-dropfilter waveguides has to be also taken into account. 


\subsection{Pump and signal powers evolution inside the active MRR}

We assume that the resonance condition ( $\beta L=2 m \pi$, where $\mathrm{m}$ is an arbitrary integer) is fulfilled for both the pump and signal wavelengths and analyse the evolution of the pump and signal powers inside the MRR.

Then, to determine the intensity rates in Eqs. (12) - (15) not only the passive characteristics of the microring resonator (losses, coupling and transmission coefficients) are required but also pump and signal gain coefficients, which depend on the active MRR working conditions. The evolution of pump and signal powers inside the resonator greatly differ. Whereas signal gain coefficient is habitually be positive even for low pump powers, pump gain coefficient is always negative since pump experiences attenuation along the ring due to absorption by the RE ions.

Pump intensity enhancement inside the ring. The pump power that circulates inside the ring is best described using the intensity enhancement factor, $E$, the rate between the confined and the input intensities, which can be evaluated as the average intensity rate ${ }^{15}$ :

$$
\begin{aligned}
& E=\frac{1}{\pi R} \int_{0}^{\pi R}\left(I_{21}+I_{31}\right) \exp [2(g-\alpha)] d z= \\
& =\frac{1}{\pi R} \int_{0}^{\pi R} \frac{\kappa_{1}^{2}\left\{1+t_{2}^{2} \delta\right\}}{\left(1-t_{1} t_{2} \delta\right)^{2}} \exp [2(g-\alpha)] d z==\frac{\kappa_{1}^{2}\left\{1+t_{2}^{2} \delta\right\}}{\left(1-t_{1} t_{2} \delta\right)^{2}} \frac{\{1-\delta\}}{(\alpha-g) L}
\end{aligned}
$$

Signal transfer functions and threshold gain coefficient. For a resonant signal the transfer functions (Eqs. (12) and (15)) become

$$
I_{11}=\left\{\frac{t_{1}-\left(1-\Gamma_{1}\right) t_{2} \delta}{1-t_{1} t_{2} \delta}\right\}^{2}, I_{41}=\left\{\frac{\kappa_{1} \kappa_{2}}{1-t_{1} t_{2} \delta}\right\}^{2} \delta
$$

The intensity rate to the through port, $I_{11}$, cancels when the critical coupling (CC) condition is verified:

$$
t_{1}=\left(1-\Gamma_{1}\right) t_{2} \delta
$$

Fulfillment of the CC condition produces the complete destructive interference between the internal field coupled into the output waveguide and the transmitted field in $c_{1}$ and, as a consequence, the transmitted intensity drops to zero. From Eq. (18), it can be concluded that if $g-\alpha>0$ (i.e. $\delta>1$ ), intensity rates $I_{11}$ and $I_{41}$ may be greater than unity and the device is a MRR amplifier. On the other hand if gain compensates all the roundtrip losses and the denominators in Eq. (18) approach zero, $I_{11}$ and $I_{41}$ tend to infinity and the oscillation condition is reached. The threshold gain coefficient, $g_{t h}$, can be calculated as: 


$$
g_{\text {th }}=\alpha_{s}-\frac{\ln \left[1-t_{1} t_{2}\right]}{2 \pi r}
$$

Finally, if $g>g_{t h}$, the MRR behaves as a laser amplifier. Therefore, the fulfillment of the oscillation condition depends on the achievable signal gain coefficient, what forces a previous optimizing design based on the active MRR working conditions.

\subsection{The $\mathrm{Yb}^{3+} / \mathrm{Er}^{3+}$-codoped system in phosphate glass}

Due to the large $\mathrm{Yb}^{3+}$ absorption cross section in the 980-nm band compared to that of $\mathrm{Er}^{3+}$ and the good overlapping between the $\mathrm{Er}^{3+}$-ion absorption spectrum $\left({ }^{4} \mathrm{I}_{15 / 2} \Rightarrow{ }^{4} \mathrm{I}_{11 / 2}\right)$ and the $\mathrm{Yb}^{3+}$-ion emission spectrum $\left({ }^{2} \mathrm{~F}_{5 / 2} \Rightarrow{ }^{2} \mathrm{~F}_{7 / 2}\right)$, ytterbium is a good sensitizer to efficiently improve the gain performance of $\mathrm{Er}^{3+}$-doped waveguide amplifiers. Moreover, because of their high solubility for RE ions and their excellent optical, physical and chemical properties, phosphate glasses stand out among all laser materials for RE-doped waveguide amplifiers and lasers. In particular, high dopant concentrations can be achieved without serious ion clustering [25].

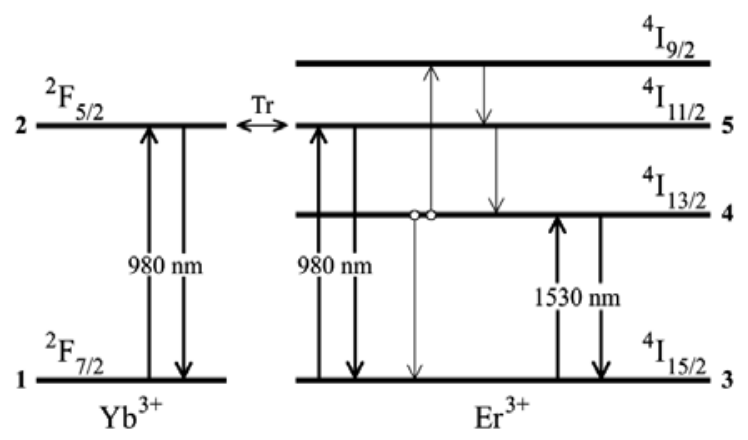

Figure 2. Energy level scheme of the $\mathrm{Yb}^{3+}-\mathrm{Er}^{3+}$-codoped system

The schematic energy level diagram of $\mathrm{aYb}^{3+} / \mathrm{Er}^{3+}$-codoped phosphate glass system is shown in Fig. 2. We assume the model for a $980-\mathrm{nm}$ pumped $\mathrm{Yb}^{3+} / \mathrm{Er}^{3+}$-codoped phosphate glass waveguide amplifier presented in Ref. [16]. In this model the temporal evolution of the population densities of the levels, $n_{i}(i=1,5)$, is described by the rate equations for the $\mathrm{Yb}^{3+} / \mathrm{Er}^{3+}$ codoped system, which can be written as follows:

$$
\begin{gathered}
\frac{d n_{2}}{d t}=W_{12} n_{1}+C_{B T} n_{1} n_{5}-\left[A_{2}+W_{21}\right] n_{2}-C_{E T}\left(n_{Y b}\right) n_{2} n_{3} \\
\frac{d n_{4}}{d t}=W_{34} n_{3}-\left[A_{4}+W_{43}\right] n_{4}-2 C_{U P}\left(n_{4}\right) n_{4}^{2}+A_{5} n_{5}
\end{gathered}
$$




$$
\begin{gathered}
\frac{d n_{5}}{d t}=-C_{B T} n_{1} n_{5}+C_{E T}\left(n_{Y b}\right) n_{2} n_{3}+W_{35} n_{3}+C_{U P}\left(n_{4}\right) n_{4}^{2}-\left[A_{5}+W_{53}\right] n_{5} \\
n_{1}+n_{2}=n_{Y b} \\
n_{3}+n_{4}+n_{5}=n_{E r}
\end{gathered}
$$

where the population densities of the ytterbium ion levels ${ }^{2} \mathrm{~F}_{7 / 2}$ and ${ }^{2} \mathrm{~F}_{5 / 2}$, and of the erbium ion levels ${ }^{4} \mathrm{I}_{15 / 2},{ }^{4} \mathrm{I}_{13 / 2}$ and ${ }^{4} \mathrm{I}_{11 / 2}$, are $n_{1}(x, y, z), n_{2}(x, y, z), n_{3}(x, y, z), n_{4}(x, y, z)$ and $n_{5}(x, y, z)$, respectively. Notice that, for the sake of simplicity, in Eqs. (21) - (25), the spatial dependence $(x, y, z)$ of the population densities and the densities of stimulated radiative transition rates is omitted. Furthermore, $n_{Y b}$ and $n_{E r}$ denote the homogeneous ytterbium and erbium ions concentrations. In Eqs. (21)-(25), $A_{i}$ represents the spontaneous relaxation rate from level $i$, whereas the values of the densities of stimulated radiative transition rates, $W_{i j}(x, y, z)$, can be obtained using the equation

$$
W_{i j}(x, y, z)=\sum_{v} \frac{\sigma_{i j}(v)}{h v} \Psi(x, y, v) \times P(z, v)
$$

where $\Psi(x, y, v)$ is the normalized mode envelope [26] of the pump, signal or co- and counterpropagating amplified spontaneous emission (ASE ${ }^{ \pm}$) waves, with optical frequency $v$. $\Psi(x, y, v)$ is assumed to be $z$-independent and depends on the index profile and waveguide profile and the waveguide geometry. Besides, in Eq. (26), $P(z, v)$ are the z-propagating total optical powers and $\sigma_{i j}(v)$ are the absorption/emission cross sections corresponding to the transition between the $i t h$ and $j$ th levels. Concerning the energy-transfer inter-atomic mechanisms, a term proportional to the $\mathrm{Er}^{3+}$-ion first excited level population squared is used to describe the upconversion effect. The homogeneous upconversion coefficient (HUC) assesses the number of upconversion events per unit time and is a function of the first excited level population, $C_{U P}\left(n_{4}\right)$. Finally, the $\mathrm{Yb}^{3+} \Rightarrow \mathrm{Er}^{3+}$ energy transfer and back transfer coefficients are $C_{E T}\left(n_{Y b}\right)$ and $C_{B T}$, respectively.

We use available parameters from measurements on $\mathrm{Yb}^{3+} / \mathrm{Er}^{3+}$-codoped phosphate glass in order to numerically evaluate the $\mathrm{Yb}^{3+} / \mathrm{Er}^{3+}$-codoped system rate equations. In particular, the fluorescence lifetime of the $\mathrm{Yb}^{3+}$-ion level ${ }^{2} \mathrm{~F}_{5 / 2}$ is assumed to be $1.1 \mathrm{~ms}$ [27], that of the $\mathrm{Er}^{3+}$-ion levels ${ }^{4} \mathrm{I}_{13 / 2}$ and ${ }^{4} \mathrm{I}_{11 / 2}$ are $7.9 \mathrm{~ms}[28]$ and $3.6 \times 10^{5} \mathrm{~s}^{-1}$ [29], respectively. Both absorption and emission cross-section distributions for the 1535-nm band are taken from Ref. [30], and the 976nm pump laser cross sections are taken from Ref. [28] for both ions. According to Ref. [16], for weak CW pump, the HUC coefficient is nearly a constant, whereas for high pump range it is a non-quadratic function of $n_{4}(x, y, z)$ and saturates at the kinetic limit in the case of infinite pump power [31]. As the $\mathrm{Er}^{3+}$ ion concentration increases, the upconversion coefficient also increases due to the migration contribution. This formalism was recently adapted to include 
$\mathrm{Yb}^{3+}$-sensitization and transversally resolved rate equations, which become essential due to the nonlinear character of the energy transfer mechanisms.

Concerning the $\mathrm{Yb}^{3+} \Rightarrow \mathrm{Er}^{3+}$ energy-transfer rate, we assume the fitted values to an experimental dependence of the energy transfer coefficient in [32]. Finally, since the population in the $\mathrm{Er}^{3+}-$ ion level ${ }^{4} \mathrm{I}_{11 / 2}$ remains low even at high pump powers, in practice, the value of the $\mathrm{Er}^{3+} \Rightarrow$ $\mathrm{Yb}^{3+}$ back transfer coefficient can be assumed as a constant, $C_{B T}=1.5 \times 10^{-22} \mathrm{~m}^{3} / \mathrm{s}$ [33].

\begin{tabular}{lcc}
\hline \multicolumn{1}{c}{ Parameter } & Symbol & Value \\
\hline Signal wavelength & $\lambda_{s}$ & $1534 \mathrm{~nm}$ \\
\hline Pump wavelength & $\lambda_{p}$ & $976 \mathrm{~nm}$ \\
\hline Decay rate of $\mathrm{Yb}^{3+2} F_{5 / 2}$ & $A_{2}$ & $909 \mathrm{~s}^{-1}$ \\
\hline Decay rate of $\mathrm{Er}^{3+4} \mathrm{I}_{13 / 2}$ & $A_{4}$ & $127 \mathrm{~s}^{-1}$ \\
\hline Decay rate of Er ${ }^{3+4} \mathrm{I}_{11 / 2}$ & $A_{5}$ & $3.6 \times 10^{5} \mathrm{~s}^{-1}$ \\
\hline Absorption cross section $\mathrm{Yb}^{3+2} \mathrm{~F}_{7 / 2}$ at $\lambda_{p}$ & $\sigma_{12}$ & $10.9 \times 10^{-25} \mathrm{~m}^{2}$ \\
\hline Emission cross section $\mathrm{Yb}^{3+2} \mathrm{~F}_{5 / 2}$ at $\lambda_{p}$ & $\sigma_{21}$ & $11.6 \times 10^{-25} \mathrm{~m}^{2}$ \\
\hline Absorption cross section $\mathrm{Er}^{3+4} \mathrm{I}_{15 / 2}$ at $\lambda_{p}$ & $\sigma_{35}$ & $1.5 \times 10^{-25} \mathrm{~m}^{2}$ \\
\hline Emission cross section $\mathrm{Er}^{3+4} \mathrm{I}_{11 / 2}$ at $\lambda_{p}$ & $\sigma_{53}$ & $9.6 \times 10^{-26} \mathrm{~m}^{2}$ \\
\hline Absorption cross section $\mathrm{Er}^{3+4} \mathrm{I}_{15 / 2}$ at $\lambda_{s}$ & $\sigma_{34}$ & $5.4 \times 10^{-25} \mathrm{~m}^{2}$ \\
\hline Emission cross section $\mathrm{Er}^{3+4} \mathrm{I}_{13 / 2}$ at $\lambda_{s}$ & $\sigma_{43}$ & $5.3 \times 10^{-25} \mathrm{~m}^{2}$ \\
\hline Energy transfer rate $\mathrm{Er}^{3+} \Rightarrow \mathrm{Yb}^{3+}$ & $C_{B T}$ & $1.5 \times 10^{-22} \mathrm{~m}^{3} / \mathrm{s}$ \\
$\left({ }^{4} \mathrm{I}_{11 / 2}+{ }^{2} \mathrm{~F}_{7 / 2} \Rightarrow{ }^{4} \mathrm{I}_{15 / 2}+{ }^{2} \mathrm{~F}_{5 / 2}\right)$ & $R_{u}$ & $9.95 \AA$ \\
\hline Upconversion critical radius & $R_{m} / R_{u}$ \\
\hline Ratio between critical $\mathrm{radii}^{1 / 6}$ & & \\
\hline
\end{tabular}

Table 1. Parameters used for the gain calculations

\subsection{Propagation of the optical powers}

The evolution along the active waveguide of the pump, signal and ASE powers can be expressed as follows:

$$
\begin{gathered}
\frac{d P_{p}\left(z, v_{p}\right)}{d z}=\sigma_{53}\left(v_{p}\right) N_{5}\left(z, v_{p}\right)-\sigma_{35}\left(v_{p}\right) N_{3}\left(z, v_{p}\right)+\sigma_{21}\left(v_{p}\right) N_{2}\left(z, v_{p}\right)-\sigma_{12}\left(v_{p}\right) N_{1}\left(z, v_{p}\right)-\alpha\left(v_{p}\right) \\
\frac{d P_{s}\left(z, v_{s}\right)}{d z}=\sigma_{43}\left(v_{s}\right) N_{4}\left(z, v_{s}\right)-\sigma_{34}\left(v_{s}\right) N_{3}\left(z, v_{s}\right)-\alpha\left(v_{s}\right)
\end{gathered}
$$


In Eqs. (27) and (28), $P_{\gamma}^{ \pm}\left(z, v_{\gamma}\right)$ are the optical powers, where $\mathrm{z}$ is the distance along the waveguide axis and the label $\gamma$ is $p$ for pumping and $s s$ for signal. The pump and the signal are assumed to be monochromatic and the wavelength-dependent scattering losses are denoted as $\alpha\left(v_{\gamma}\right)$ and their $\lambda$ dependence is assumed to follow Rayleigh $\lambda^{-4}$ law. Finally, in Eqs. (27) - (28) the coupling parameters, $N_{i}\left(z, v_{s}\right)$, are the overlapping integrals between the normalized intensity modal and the $i$ th level population density distributions over $\mathrm{A}$, which is the active area,

$$
N_{i}(z, v)=\iint_{A} \Psi(x, y, v) n_{i}(x, y, z) d x d y
$$

In Eq. (29), A is defined as the area for which the integral of the addition of population densities of the excited levels converges within a required precision. Finally, a Runge-Kutta-based iterative procedure can be used to numerically integrate the equations that describe the propagation of the optical powers along the waveguide, Eqs. (27) and (28).

\section{Numerical analysis of an active add-drop filter}

\subsection{Passive structure}

An air-cladded ridge guiding structure, which presents attractive features for sensing applications [34], has been adopted for the calculations. In Table 2 we summarize the passive parameters of the structure.

\begin{tabular}{|c|c|}
\hline Parameter & Value \\
\hline Waveguide cross section & $1.5 \mu \mathrm{m} \times 1.5 \mu \mathrm{m}$ \\
\hline Substrate refractive index & 1.51 \\
\hline Core refractive index & 1.65 \\
\hline Pump wavelength & $976 \mathrm{~nm}$ \\
\hline Signal wavelength & $1534 \mathrm{~nm}$ \\
\hline Pump mode confinement factor & 0.962 \\
\hline Signal mode confinement factor & 0.757 \\
\hline Microring radius & $15.47 \mu \mathrm{m}$ \\
\hline Pump wavelength resonant order & 156 \\
\hline Signal wavelength resonant order & 96 \\
\hline Propagation loss amplitude coefficient & $0.25 \mathrm{~dB} / \mathrm{cm}$ \\
\hline
\end{tabular}

Table 2. Passive parameters of the structure

The amplitude coupling ratios for pump and signal at each coupler are functions of $d_{i}(i=1,2)$. In Fig. 3, we plot the ratios evaluated according to Ref. [35]. Particularly, for the more confined pump power a limited range of values is available. 


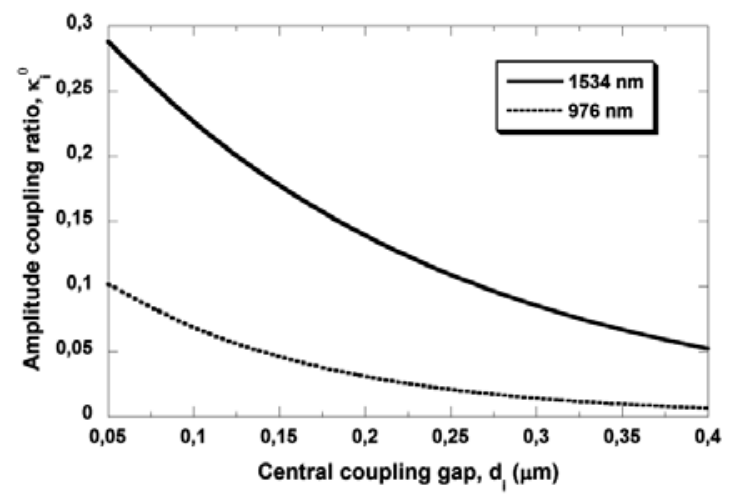

Figure 3. Pump $(\lambda=976 \mathrm{~nm})$ and signal $(\lambda=1534 \mathrm{~nm})$ amplitude coupling ratios as a function of $d_{i}(i=1,2)[21]$.

When additional coupling losses are included in the model, the practical range of central coupling gap and accordingly of the amplitude coupling ratio will be further limited. For our analysis the range of additional coupling losses is estimated from Ref. [36], where the value 0.014 is obtained for $d=117 \pm 5 \mathrm{~nm}$. When the coupling gap between the MRR and the access waveguide is below this value they report a significant increase of these losses.

\subsection{Pump enhancement inside the microring}

Besides the lossless amplitude coupling coefficient, $\kappa_{p}^{0}$, and the additional pump coupling loss, $\Gamma_{p}$, the pump intensity enhancement factor, $E_{p}$, is basically determined by the pump amplitude gain coefficient, $g_{p}$, which reflects the attenuation induced on the pump power by the stimulated transitions in the RE ions. In Fig. $4, E_{p}$ is plotted as a function of $\kappa_{p}^{0}$ for 5 values of $g_{p}$ with $\Gamma_{p}=0.005$. As the pump is more attenuated (the absolute value of $g_{p}$ increases), the maximum $E_{p}$ diminishes and is obtained for larger $\kappa_{p}^{0}$.

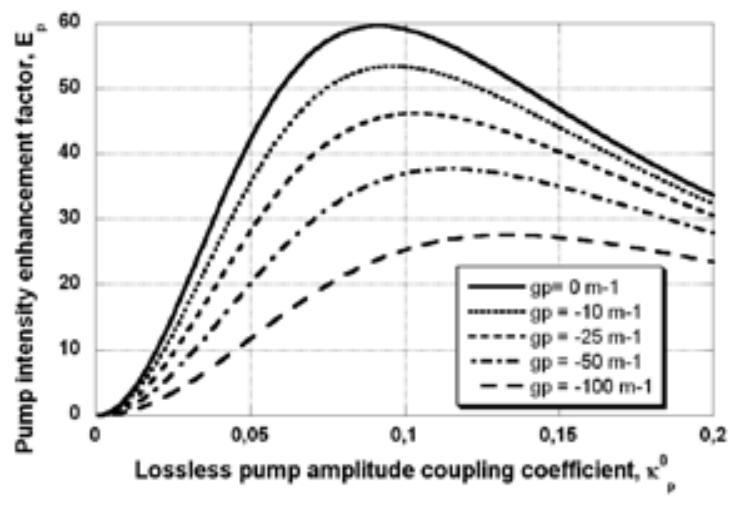

Figure 4. Pump enhancement factor as a function of the lossless amplitude coupling coefficient, $\kappa_{p}^{0}$, for different values of the pump gain coefficient [20]. 
Then, using the equations for the coupled evolution of the population densities and optical powers we have calculated the pump amplitude gain coefficient in a waveguide with $L=97.20$ $\mu \mathrm{m}(2 \pi \times 15.47 \mu \mathrm{m})$ as a function of the average circulating pump power. This dependence is plotted in Fig. 5 for five concentration pairs $\left(n_{Y b}, n_{E r}\right)$ where concentration units are $1 \times 10^{26}$ ions $/ \mathrm{m}^{3}$. RE ions concentration values were chosen with $n_{Y b}=2 n_{E r}$, since this rate is often used experimentally. The amplitude pump gain coefficient varies greatly with the average circulating pump power inside the ring. Low pump powers are strongly attenuated as the dopant concentration increases whereas high pump powers are relatively less affected by rare earth absorption.

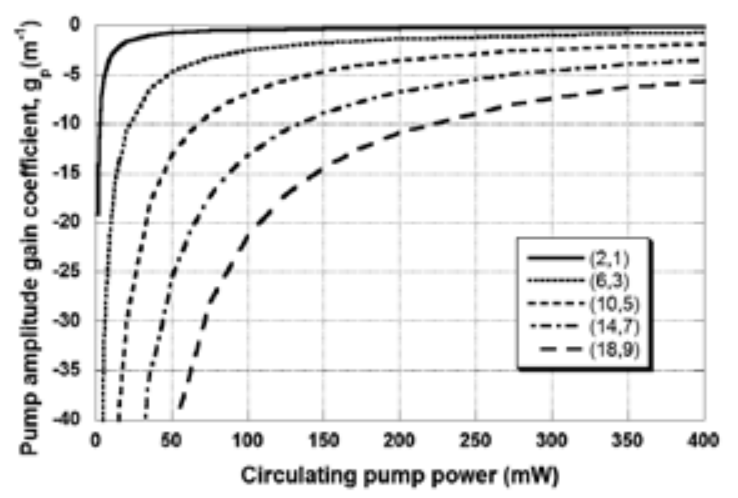

Figure 5. Pump amplitude gain coefficient in a lossless waveguide as a function of the average circulating pump power for five concentration pairs $\left(n_{Y b^{\prime}}, n_{E r}\right)$. Units for the RE concentrations are $1 \times 10^{26}$ ions $/ \mathrm{m}^{3}$ [20].

As shown in Fig. 4 and $5, g_{p}$ depends on the circulating pump power but, in its turn, $E_{p}$ is a function of $g_{p}$. In practice, for given concentration values, if the required average circulating pump to achieve a signal gain coefficient value is calculated, the associated $g_{p}$ can be determined, and subsequently, the pump intensity enhancement and the necessary input pump power.

\subsection{Signal gain coefficient}

First, as with the pump intensity enhancement, we analyse the dependence of the signal intensity rate between the drop and the input ports, $I_{41}$, on the lossless coupling and on the signal gain coefficient. In Fig. $6, I_{41}$ is plotted as a function of the signal gain coefficient for four values of the lossless amplitude coupling coefficient and $\Gamma_{p}=0.005$.

For each value of $\kappa_{s}^{0}, I_{41}$ does not grow significantly until $g_{s}$ approaches the threshold gain (when $I_{41}$ tends to infinity). Then, the input signal is strongly amplified and the rate of growth of $I_{41}$ is higher for lower $\kappa_{s}^{0}$. Over the gain threshold laser operation is achieved. As we did with $g_{p}$, we now calculate $g_{s}$ as a function of the circulating pump power for five pairs of dopant concentrations. 


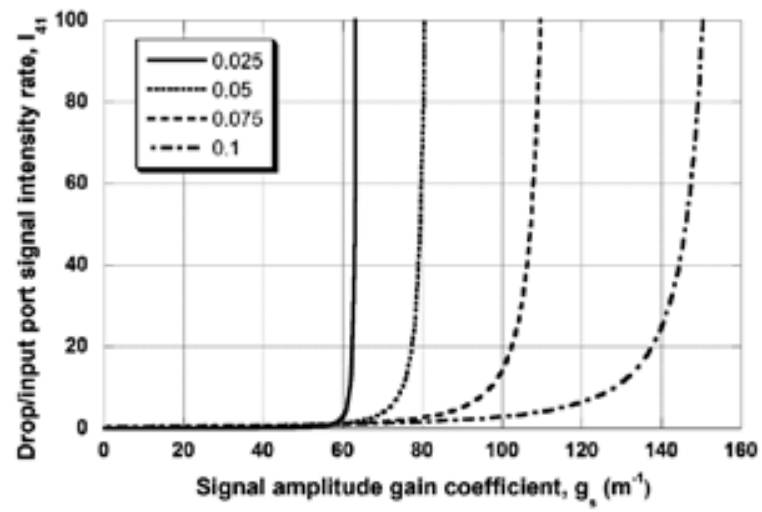

Figure 6. Intensity rate between the drop and the input ports as a function of the signal gain coefficient for four values of the lossless amplitude coupling coefficient $\kappa_{s}^{0}[20]$.

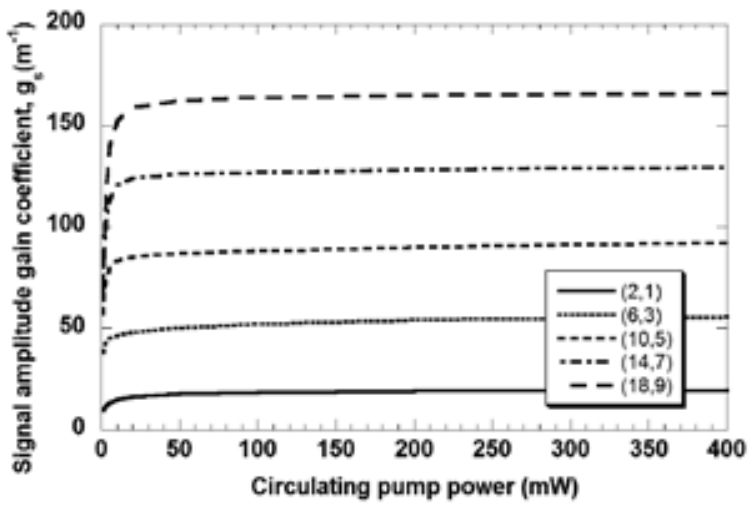

Figure 7. Signal amplitude gain coefficient in a lossless waveguide as a function of the average circulating pump power for 5 concentration pairs $\left(n_{Y b}, n_{E r}\right)$. Units for the RE concentrations are $1 \times 10^{26}$ ions $/ \mathrm{m}^{3}$ [20].

As it can be appreciated in Fig. $7, g_{s}$ saturates for relatively low circulating pump power for any RE concentration pair. This is caused by the short MRR length, which is much shorter than the waveguide amplifier optimal lengths for each pump power and RE ions concentrations.

By comparing Figs. 6 and 7, the minimum RE ions concentrations necessary to achieve a significant amplification can be estimated as a function of $\kappa_{s}^{0}$. For instance, if $\kappa_{s}^{0}=0.05$, amplification becomes significant for $g_{s} \approx 80 \mathrm{~m}^{-1}$. However, to achieve this gain, a high doping level is mandatory, $n_{Y b}=10 \times 10^{26}$ ions $/ \mathrm{m}^{3}$ and $n_{E r}=5 \times 10^{26}$ ions $/ \mathrm{m}^{3}$, approximately. Except for low values $(<10 \mathrm{~mW})$, the circulating pump power has a small influence on $g_{s}$. For larger values of $\kappa_{s}^{0}$ the requirement for high doping level is more and more demanding. Therefore, in practice, the available RE doping level limits the value of $\kappa_{s}^{0}$ for an amplifying MRR and the range of $\mathrm{d}$ and the corresponding $\kappa_{p}^{0}$. For $\kappa_{s}^{0}=0.05$, the central coupling gap is $d \approx 0.4 \mu \mathrm{m}$ and $\kappa_{p}^{0} \approx 0.006$. According to Ref. [36], for this value of $d$, low additional coupling losses both for pump and 
signal could be feasible. Once $\kappa_{p}^{0}$ is determined, from Fig. 4 and depending on $\Gamma_{p}$ and $g_{p}$, the pump intensity enhancement factor, $E_{p}$, is obtained and, subsequently, the pump power that has to be the input in the MRR. Although the circulating pump power had a small influence in $g_{s}$, together with the RE ions concentrations, determines $g_{p}$ (see Fig. 5) and $E_{p}$.

\section{Gain/oscillation requirements for a symmetric structure}

\subsection{Net gain requirements for a symmetric structure}

Firstly, the requirements to achieve net gain and oscillation are going to be analysed in a symmetric structure and afterwards we extend this analysis to asymmetric structures. Therefore, in Sections 4.1 and 4.2, equal lossless amplitude coupling ratios for both pump and signal powers between the microring and the straight waveguides $\left(\kappa_{\lambda}^{0}=\kappa_{1, \lambda}^{0}=\kappa_{2, \lambda}^{0}\right)$ and additional coupling losses $\left(\Gamma_{\lambda}=\Gamma_{1, \lambda}=\Gamma_{2, \lambda}\right)$ are considered. The net gain that can be obtained in the MRR amplifier is evaluated as:

$$
\text { Net Gain }(d B)=10 \log \left(I_{41}\right)
$$

Net gain dependence on $g_{s}$ is plotted in Fig. 8 for three values of $\kappa_{s}^{0}$ and (a) $\Gamma_{s}=0.005$ and (b) $\Gamma_{s}=0.01$.
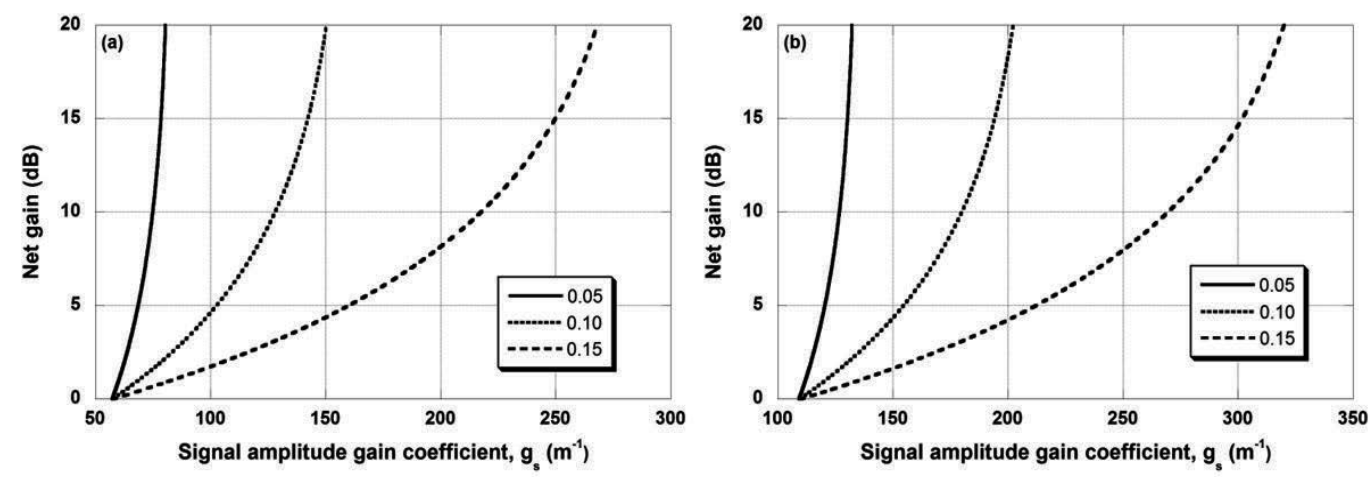

Figure 8. Net gain as a function of $g_{s}$ for three values of $\kappa_{s}^{0}$, for (a) $\Gamma_{s}=0.005 \mathrm{y}(\mathrm{b}) \Gamma_{s}=0.01$ [21].

As the additional losses increase, the value of $g_{s}$ (and accordingly of the RE ion concentrations) necessary to achieve positive gain becomes larger. For instance, if $\Gamma_{s}=0.01$, then $g_{s}>100 \mathrm{~m}^{-1}$, which implies $n_{E r}>7 \times 10^{26} \mathrm{~m}^{-3}$. Once positive net gain is achieved, the rate of growth is higher with lower $\kappa_{s}^{0}$. 


\subsection{Threshold gain and oscillation requirements for a symmetric structure}

Then, we are going to analyse the oscillation requirements. In Fig. 9, the evolution of the threshold gain as a function of the lossless amplitude coupling coefficient, $\kappa_{s}^{0}$, for different values of the additional coupling losses is plotted. The great influence on these requirements of $\Gamma_{s}$ is clearly appreciated in this figure. As an example, if $\kappa_{s}^{0}=0.05$, the threshold signal gain coefficient rapidly increases with $\Gamma_{s}$ and for $\Gamma_{s}=0,0.005,0.01$ and 0.015 , we obtain $31.5 \mathrm{~m}^{-1}$, $83.1 \mathrm{~m}^{-1}, 134.9 \mathrm{~m}^{-1}$ and $187.0 \mathrm{~m}^{-1}$, respectively. Hence, in order to achieve the necessary $g_{t h}$, even small defects in the couplers fabrication process could only be compensated by notably raising the RE doping level. It has to be emphasized that the unavoidable requirements of high RE concentrations impose a host material with a high solubility for RE ions, as phosphate glass where high dopant concentration can be achieved without serious ion clustering [25].

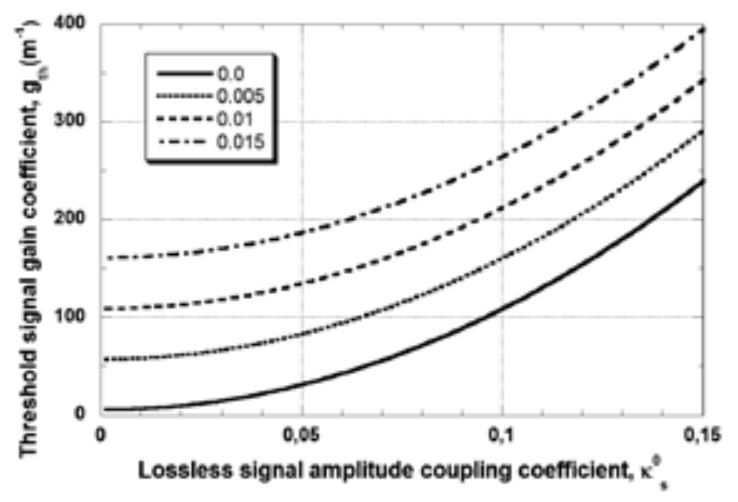

Figure 9. Threshold signal gain coefficient, $g_{t h}$, as a function of the lossless amplitude coupling coefficient, $\kappa_{s}^{0}$, for four different values of the additional coupling losses $\Gamma_{s}[21]$.

A further optimization of the structure could be accomplished if non-symmetric schemes are considered. In the next section we analyse the gain/oscillation requirements when different values for the lossless amplitude coupling ratios and additional coupling losses between each straight waveguide and the microring are allowed.

\section{Gain/oscillation requirements for an asymmetric structure}

In order to parameterize the asymmetry of the structure, we use the relative variation of the lossless amplitude coupling coefficient, $\Delta \kappa_{r, \lambda}^{0}$, that is defined as $\Delta \kappa_{r, \lambda}^{0}=\left(\kappa_{2, \lambda}^{0}-\kappa_{1, \lambda}^{0}\right) / \kappa_{1, \lambda}^{0}$. We limit the relative variation between -0.2 and, for simplicity 0.2 , we assume the same additional coupling losses for both couplers. A particular attention is going to be paid to active criticallycoupled structures and to compare their performance to the passive ones. 


\subsection{Asymmetry influence on pump enhancement}

Pump enhancement presents a maximum as a function of $\kappa_{p}^{0}$ for each $\Gamma_{p}$ in a symmetric structure for a given value of $g_{p}$. This maximum shifts towards higher $\kappa_{1, p}^{0}$ values and rapidly decreases as additional losses increase [20].
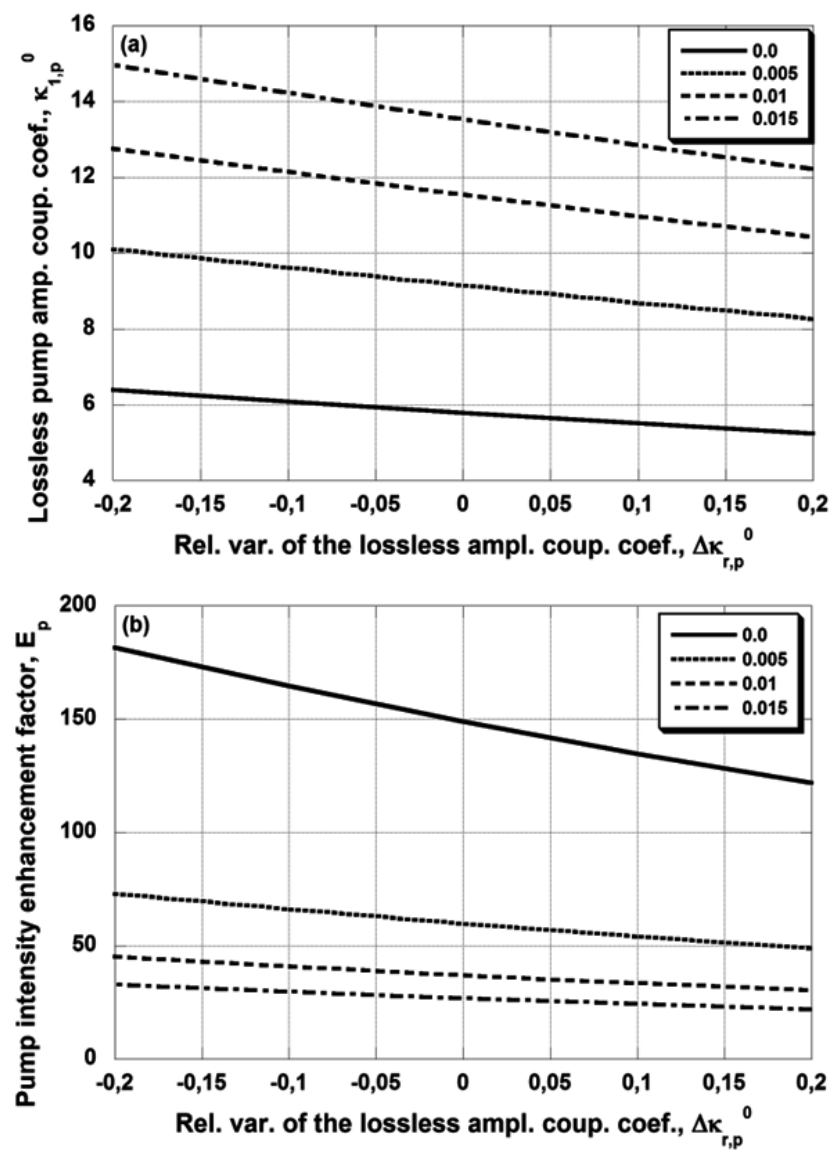

Figure 10. Evolution of the position and value of the pump enhancement maxima as a function of $\Delta \kappa_{r, p}^{0}$ for different values of $\Gamma_{1, s}:$ (a) $\kappa_{1, p}^{0}$ and (b) $E_{p}[21]$.

In Fig. 10, the evolution of the maxima position and value are represented as a function of $\Delta \kappa_{r, p}^{0}$ for different values of $\Gamma_{1, s}$. It is clear from Fig. 6 that $\Delta \kappa_{r, p}^{0}>0$ (maximum value shifts towards lower $\kappa_{p}^{0}$ ) favours pump enhancement (in the limited range of values achievable for $\kappa_{p}^{0}$ in Fig. 2). The effect of the maximum value reduction is attenuated by the saturation of small signal gain coefficient even for low circulation pump power in Fig. 3(b). 


\subsection{Asymmetry influence on the drop/input port intensity rate, $I_{41}$}

As we did with symmetric structures, net gain for asymmetric MMR is calculated. In Fig. 11, the evolution with $\Delta \kappa_{r, s}^{0}$ of the dependence of net gain with $g_{s}$ for $\kappa_{1, s}^{0}=0.1$ and $\Gamma_{1, s}=0.005$ is plotted. Although the minimum value of $g_{s}$ does not change, the rate of growth is larger for $\Delta \kappa_{r, s}^{0}<0$.

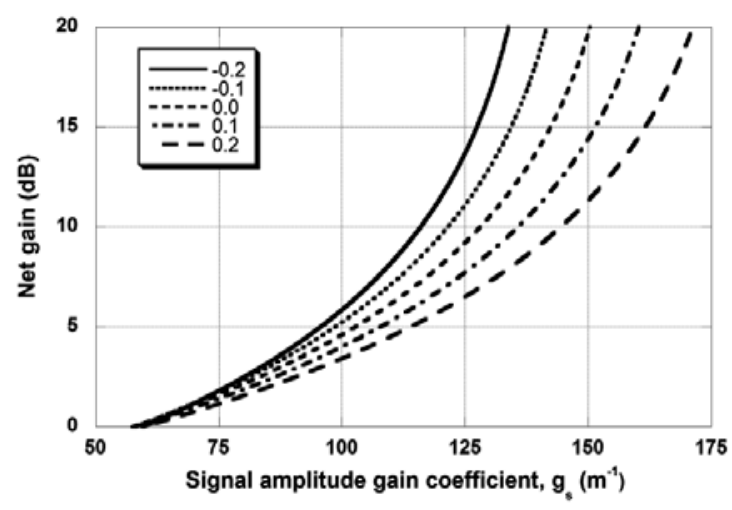

Figure 11. Evolution with $\Delta \kappa_{r, s}^{0}$ of the dependence of net gain with $g_{s}$ for $\kappa_{1, s}^{0}=0.1$ and $\Gamma_{1, s}=0.005$ [21].

Next, we study the performance in CC conditions. Differently from the passive MRR, in an active structure the value of $\kappa_{2, s}^{0}$ that cancels the throughout intensity depends on the additional losses and on the signal gain amplitude coefficient for a given $\kappa_{1, s}^{0}$.
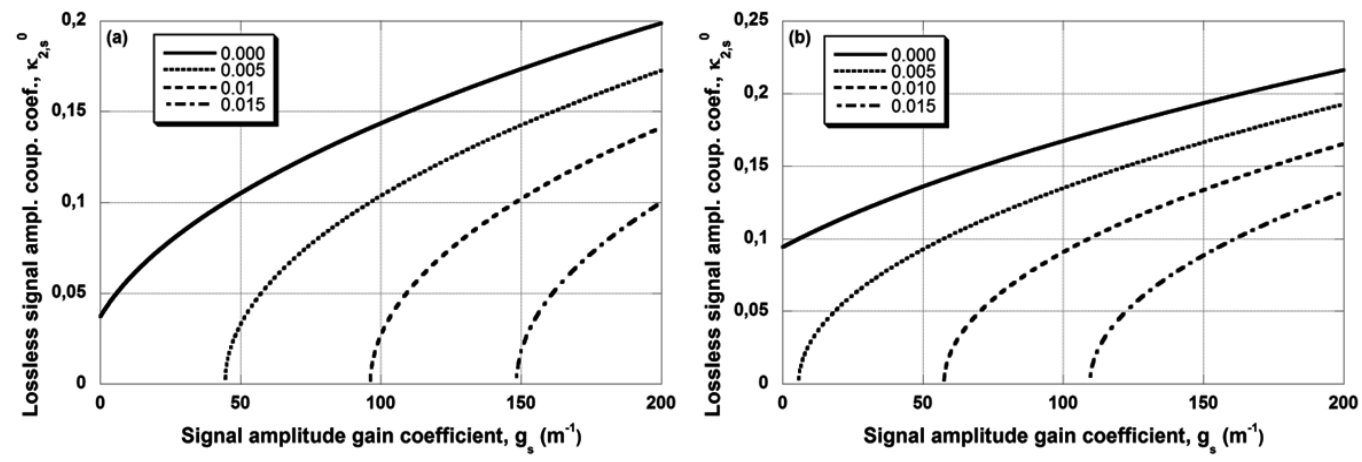

Figure 12. Lossless signal amplitude coupling coefficient $\kappa_{2, s}^{0}$ for CC as a function of $g_{s}$ for (a) $\Gamma_{1, s}=0.005$ and (b) $\Gamma_{1, s}=0.01$, for four values of $\kappa_{1, s}^{0}$ [21].

In Fig. 12, the values of $\kappa_{2, s}^{0}$ for CC are plotted as a function of $g_{s}$ for four values of $\kappa_{1, s}^{0}$ and for (a) $\Gamma_{1, s}=0.005$ and (b) $\Gamma_{1, s}=0.01$. Unlike the passive structure, performance output in the drop 
port is not maximized for CC. The net gain that can be obtained with the parameters used in Fig. 11 is plotted in Figs. 13(a) and 13(b). Although lower net gain can be attained compared with other asymmetric configurations (see Fig. 11), significant net gain can still be achieved in case the through contribution has to be minimized.
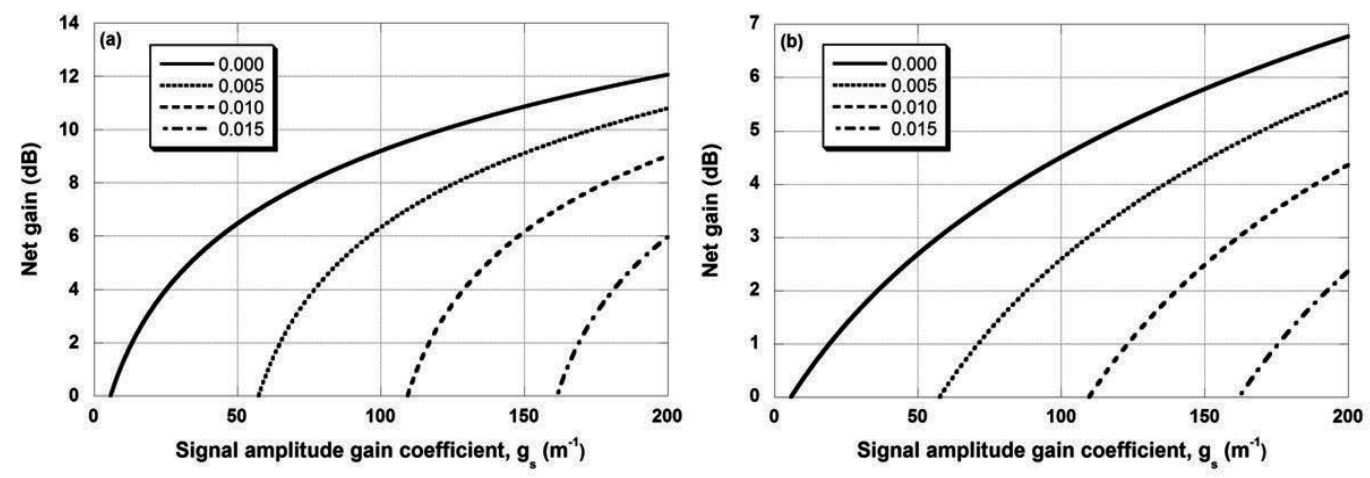

Figure 13. Net gain obtainable as a function of $g_{s}$ for the asymmetric CC configurations considered in Fig.12 for (a) $\Gamma_{1, s}=0.005$ and (b) $\Gamma_{1, s}=0.01$, for four values of $\kappa_{1, s}^{0}[21]$.

\subsection{Asymmetry influence on threshold gain}

Finally, changes in $g_{\text {th }}$ are analysed when asymmetric configurations are considered. Values of $g_{t h}$ are plotted as a function of $\Delta \kappa_{r, s}^{0}$ for different combinations of $\left(\kappa_{1, s^{\prime}}^{0}, \Gamma_{1, s}\right)$ in Fig. 14.

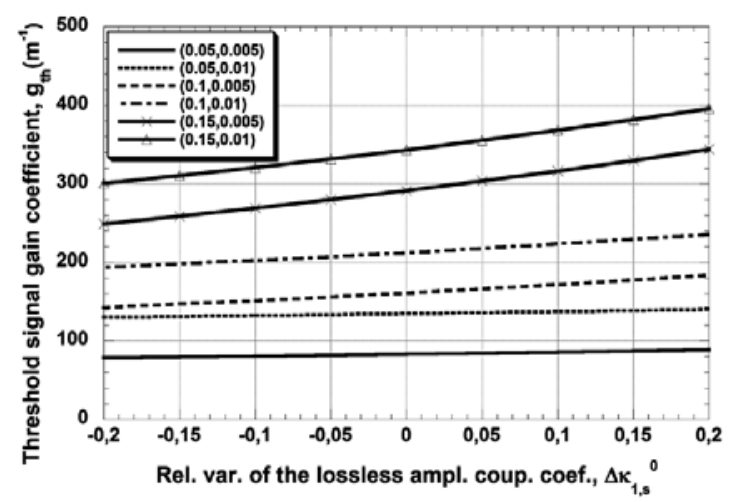

Figure 14. Variations in the threshold signal gain coefficient, $g_{t h}$, as a function of $\Delta \kappa_{r, s}^{0}$ for different combinations of $\left(\kappa_{1, s^{\prime}}^{0}, \Gamma_{1, s}\right)[21]$.

In Fig. 14 we can see how the necessary threshold gain value decreases for $\Delta \kappa_{r, s}^{0}<0$. This reduction is more significant for the higher additional coupling losses and contributes to relax the requirement for very high dopant concentrations. 


\section{Conclusions}

In order to optimize RE-doped amplifying/oscillating MRRs, the coupled evolution of resonant pump and signal powers inside the integrated structure must be modelled and the interrelated passive and active characteristics must be taken into consideration. The RE ions concentration sets the attainable signal gain coefficient. This coefficient, together with the pump intensity enhancement dependences, determines the suitable combination of passive parameters (greatly influenced by the expected additional coupling losses) and RE ions doping level to achieve significant amplification or oscillation operation.

A further optimization could be achieved if non-symmetric structures are considered, allowing different values for the lossless amplitude coupling ratios and the additional coupling losses between the microring and the straight waveguides. The use of asymmetric structures can to some extent relieve the demand of a much higher signal gain coefficient and threshold gain (and accordingly dopant concentrations) as the additional losses increase. Structures with lower output coupler coupling coefficient than the input coupler one are preferable. Finally, since signal gain saturation is achieved for relatively low circulating pump powers (due to the short length of the MRR), in practice, asymmetry has little influence on pump enhancement.

\section{Acknowledgements}

This work was partially supported by the Spanish Ministry of Economy and Competitiveness under the FIS2010-20821 and TEC2013-46643-C2-2-R projects, by the Diputación General de Aragón, el Fondo Social Europeo and by a grant of the Romanian National Authority for Scientific Research, CNDIUEFISCDI, project number PN-II-PTPCCA-2011-71 "Integrated Smart Sensor System for Monitoring of Strategic Hydrotechnical Structures HydroSens".

\section{Author details}

Juan A. Vallés ${ }^{1^{*}}$ and R. Gălătuş²

*Address all correspondence to: juanval@unizar.es

1 Department of Applied Physics and I3A, University of Zaragoza, Zaragoza, Spain

2 Optoelectronics Group, Faculty of Electronics, Telecommunications and Information

Technology, Technical University of Cluj-Napoca, Cluj-Napoca, Romania 


\section{References}

[1] Okamoto $\mathrm{H}$, Haraguchi $\mathrm{M}$ and Okamoto T. Filtering characteristic of a microring resonator with a gap. Electron. Commun. Jpn. Part II-Electron. 2006;89:25-32.

[2] Little BE, Chu ST, Absil PP, Hryniewicz JV, Johnson FG, Seiferth F et al. Very highorder microring resonator filters for WDM applications. IEEE Photonics Technol. Lett. 2004;16:2263-2265.

[3] Foerst M, Niehusmann J, Plotzing T, Bolten J, Wahlbrink T, Moormann C et al. Highspeed all-optical switch in ion-implanted silicon-on-insulator microring resonators. Opt. Lett. 2007;32:2046-2048.

[4] Balakrishnan M, Faccini M, Diemeer MBJ, Klein EJ, Sengo G, Driessen A et al. Microring resonator based modulator made by direct photodefinition of an electro-optic polymer. Appl. Phys. Lett. 2008;92:153310.

[5] Ramachandran A, Wang S, Clarke J, Ja S, Goad D, Wald L et al. A universal biosensing platform based on optical micro-ring resonators. Biosens. Bioelectron. 2008;23:939-944.

[6] Suter JD and Fan X. Overview of the optofluidic ring resonator: a versatile platform for label-free biological and chemical sensing. In Engineering in Medicine and Biology Society. EMBC 2009. Annual International Conference of the IEEE. 2009:10421044.

[7] Hunt HK and Armani AM. Label-free and chemical sensors. Nanoscale. 2010;2:15441559.

[8] Baaske $\mathrm{M}$ and Vollmer F. Optical resonator biosensors: molecular diagnostic and nanoparticle detection on an integrated platform. ChemPhysChem. 2012;13(2):427436.

[9] Yang G, White IM and Fan X. An opto-fluidic ring resonator biosensor for the detection of organophosphorus pesticides. Sens. Actuators, B. 2009;133(1):105-112.

[10] Sun $Y$ and Fan X. Analysis of ring resonators for chemical vapor sensor development. Opt. Express. 2008;16(14):10254-10268.

[11] Gohring JT, Dale PS and Fan X. Detection of HER2 breast cancer biomarker using the opto-fluidic ring resonator biosensor. Sens. Actuators, B.2010;146(1):226-230.

[12] Amarnath K, Grover R, Kanakaraju S and Ho PT. Electrically pumped InGaAsP-InPmicroring optical amplifiers and lasers with surface passivation. IEEE Photon. Technol. Lett. 2005;17(11):2280-2282.

[13] Hsiao HK and Winick KA. Planar glass waveguide ring resonator with gain. Opt. Express. 2007;15(26):17783-17797. 
[14] He L, Ozdemir SK, Zhu J, Kim W and Zhang L. Detecting single viruses and nanoparticles using whispering gallery microlasers. Nat. nanotechnol. 2011;6(7):428-432.

[15] Bradley JD and Pollnau M. Erbium-doped integrated waveguide amplifiers and lasers. Laser. Photonics. Rev. 2011;5(3):368-403.

[16] Vallés JA. Method for accurate gain calculation of a highly $\mathrm{Yb}^{3+} / \mathrm{Er}^{3+}$-codoped waveguide amplifier in migration-assisted upconversion regime. IEEE J. Quantum. Electron. 2011;47(8):1151-1158.

[17] Li Y, Vienne G, Jiang X, Pan X, Liu X, Gu P and Tong L. Modeling rare-earth doped microfiber ring lasers. Opt. Express. 2006;14:7073-7086.

[18] Wang YH, Ma CS, Yan X and Zhang DM. Analysis for amplifying characteristics of $\mathrm{Er}^{3+}-\mathrm{Yb}^{3+}$-co-doped microring resonators. Opt. \& Laser Technol. 2010;42:336-340.

[19] Vallés JA and Gălătuş R. Highly $\mathrm{Yb}^{3+} / \mathrm{Er}^{3+}$-codoped waveguide microring resonator optimized performance. IEEE Photonics Technol. Lett. 2013;25:457-459.

[20] Vallés JA and Galatus R. Modeling of $\mathrm{Yb}^{3+} / \mathrm{Er}^{3+}$-codoped microring resonators. Opt. Mater. 2015;41:126-130.

[21] Vallés JA and Gălătuş R. Requirements for gain/oscillation in $\mathrm{Yb}^{3+} / \mathrm{Er}^{3+}$-codopedmicroring resonators. Proc. SPIE. 2015;9359 Optical Components and Materials XII: 93591U.

[22] Vörckel A, Mönster M, Henschel W, Bolivar PH, Kurz H. Asymmetrically coupled silicon-on-insulator microring resonators for compact add-drop-multiplexers. IEEE Photonics. Technol. Lett. 2003;15(7),921-923.

[23] Geuzebroek DH and Driessen A. Ring-resonator-based wavelength filters. In Wavelength filters in fibre optics. Springer Berlin Heidelberg; 2006.p. 341-379.

[24] Zhao JH and Madsen CK. Optical filter design and analysis. NewYork: Wiley; 1999.

[25] Veasey DL, Funk DS, Peters PM, Sanford NA, Obarski GE, Fontaine N et al. Yb/Ercodoped and $\mathrm{Yb}$-doped waveguide lasers in phosphate glass. J. Non-Cryst. Solids. 2000;263-264:369-381.

[26] Desurvire E. Erbium-doped fiber amplifiers. New York: Wiley; 1994.

[27] Taccheo S, Laporta P, Longhi S, Svelto C. Experimental analysis and theoretical modeling of a diode-pumped Er: Yb: glass microchip laser. Opt. Lett. 1995;20:889-891.

[28] Vallés JA, Ferrer A, Rebolledo MA, Ruiz de la Cruz A, Berdejo V and Solis J. Study of an optimised bidirectional pump scheme for fs-laser written $\mathrm{Yb} / \mathrm{Er}$-codoped integrated waveguides. Opt. Mater. 2010;33:231-235.

[29] Honkanen S, Ohtsuki T, Jiang S, Iraj-Najafi S, Peyghambarian N. High Er concentration phosphate glasses for planar waveguide amplifiers. Proc. SPIE. 1997;2996:32-40. 
[30] Vallés JA, Rebolledo MA, Cortés J. Full characterization of packaged Er-Yb-codoped phosphate glass waveguides. IEEE J. Quantum. Electron. 2006;42:152-159.

[31] Khoptyar D, Sergeyev S and Jaskorzynska B. Homogeneous upconversion in Er-doped fibres under steady state excitation. Analytical model and its Monte-Carlo verification. J. Opt. Soc. Am. B. 2005;22(3):582-590.

[32] Gapontsev VP, Matitsin SM, Isineev AA and Kravchenko VB. Erbium glass lasers and their applications. Opt. Laser Technol. 1982;14:189-196.

[33] Majaron B, Čopič M, Lukač M and Marinček M. Influence of hole burning on laser pumping dynamics and efficiency in $\mathrm{Yb}$ : Er: phosphate glasses. Proc. SPIE. 1994;2138:183-190.

[34] Passaro VMN, Dell'Olio F and De Leonardis F. Sensors 2007;7:2741-2749.

[35] Ma CS, Yan X, Xu YZ, Qin ZK and Wang XY. Characteristic analysis of bending coupling between two optical waveguides.Opt. Quantum. Electron. 2005;37:1055-1067.

[36] Xia F, Sekaric L and Vlasov YA. Mode conversion losses in silicon-on-insulator photonic wire based racetrack resonators. Opt. Express. 2006;14:3872-3886. 



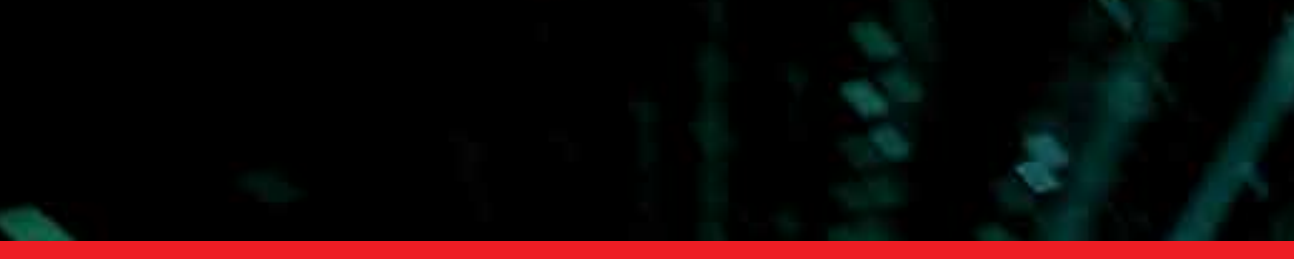

\section{Edited by Sisir Kumar Garai}

With the explosion of information traffic, the role of optical amplifiers becomes very significant in fulfilling the demand of faster optical signals and data processing in the field of communication. This book covers different advanced functionalities of optical amplifiers as well as their emerging applications in optical communication networks.

The first chapter deals with an efficient and validated time-domain numerical modelling of semiconductor optical amplifiers (SOAs) and SOA-based circuits, while the second chapter is based on the working of gallium nitride-based semiconductor optical amplifiers. The role of SOAs for the next generation of high-data-rate optical packet-switched network is presented in Chapter 3. Chapter 4 covers the all-optical semiconductor optical amplifier based on quantum dots (QD-SOA) and its function as an arithmetic processor. In Chapter 5 , the authors have presented the role of SOAs in intensity modulation of the optical pulses and their use in deterministic timing jitter and peak pulse power equalization analysis. In Chapter 6, the investigation of broadband S-band to L-band erbium-doped fibre amplifier (EDFA) module is presented, and Chapter 7 includes the optimized design technique of $\mathrm{Yb}_{3+} / \mathrm{Er}_{3+-}$ codoped phosphate microring resonator amplifiers.

All selected chapters are very interesting and well organized, and I hope they will be of great value to postgraduate students, researchers, academics and anyone seeking to understand the advanced functionalities of optical amplifiers in the present scenario. 